\title{
Diseño óptimo de antenas lente en microondas mediante métodos de simulación
}

Tesis Doctoral presentada por

Patricio Martín Gross

Para acceder al grado académico de DOCTOR EN INGENIERÍA

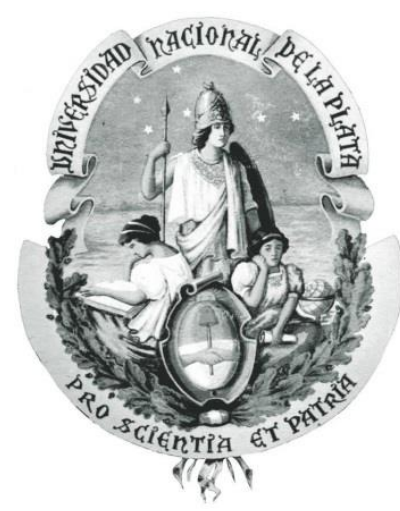

FACULTAD DE INGENIERÍA

UNIVERSIDAD NACIONAL DE LA PLATA

Director de Tesis: Dr. Ing. José Alberto Bava

Co-Director: Dr. Ing. Felipe Vico Bondia

La Plata, octubre de 2019 
Gross Patricio M. 
"The purpose of computing is insight, not numbers".

Richard Hamming 


\section{Prefacio}

Esta Tesis es presentada como parte de los requisitos para obtener el grado académico de Doctor en Ingeniería, en la Facultad de Ingeniería de la Universidad Nacional de La Plata. La misma contiene los resultados de los estudios desarrollados bajo la dirección del Dr. José Alberto Bava y la codirección del Dr. Felipe Vico Bondia. 
Gross Patricio M. 


\section{Agradecimientos}

Ante todo, mi agradecimiento a mi director de Tesis, el Dr. José Alberto Bava, sin el cual esta disertación no hubiera sido posible. Alberto ha sido un muy buen mentor desde los comienzos de mi formación profesional.

A mi compañera de trabajo, la Ing. Julieta Z. Vernieri, con quien he aprendido muchísimo habiendo realizado numerosos trabajos, muchos de los cuales son resultados de la presente Tesis.

A los doctores Miguel Ferrando Bataller y Felipe Vico Bondia de la U.P.V., España, por las numerosas charlas y consejos durante mi estadía en Valencia y por la facilitación del algoritmo de simulación empleado en muchos de los trabajos realizados.

A los Ingenieros Juan Pablo Ciafardini y Guillermo Rodríguez por sus aportes, comentarios y sugerencias durante el desarrollo de esta investigación.

A Dr. Néstor A. Bolognini por sus fundamentales aportes en el tema de Vórtices Ópticos.

A mi familia por su constante estímulo. 


\section{Contenido}

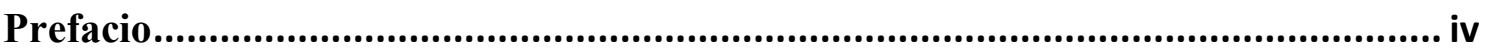

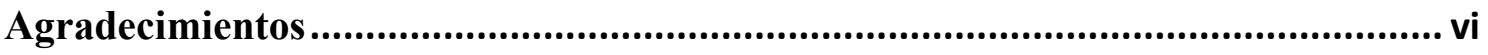

Índice de Figuras ........................................................................................ xi

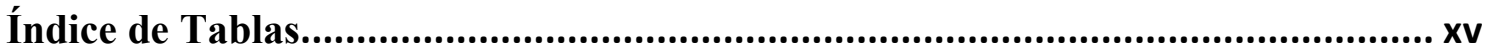

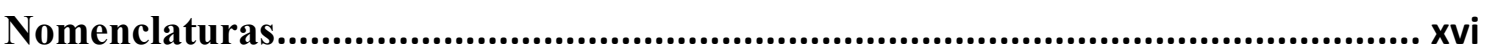

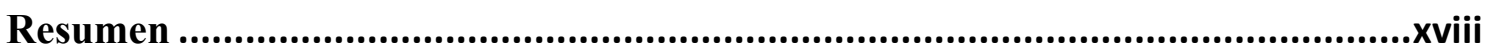

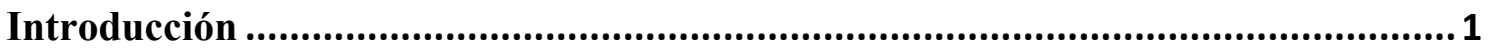

1.1 Antecedentes y motivación ......................................................................... 1

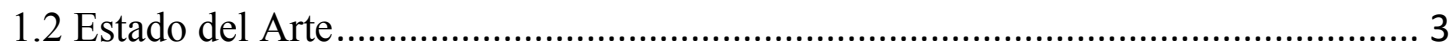

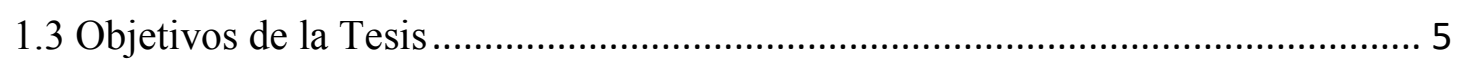

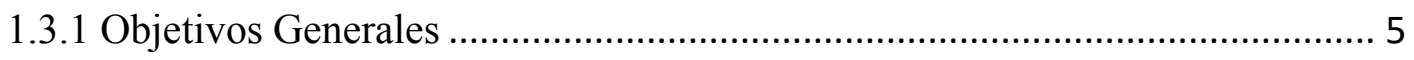

1.3.2 Objetivos Específicos......................................................................... 5

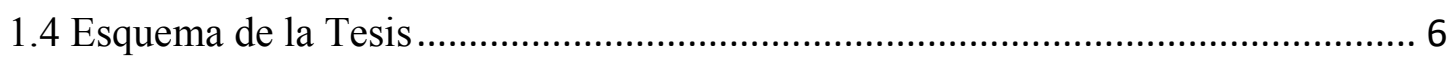

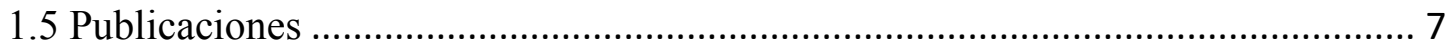

Electromagnetismo y simulación electromagnética ...........................................9

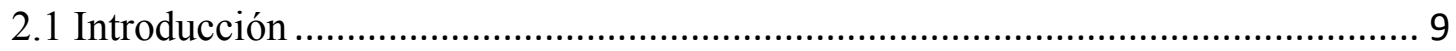

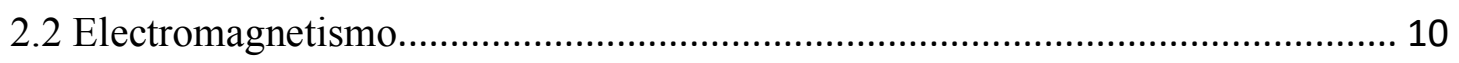

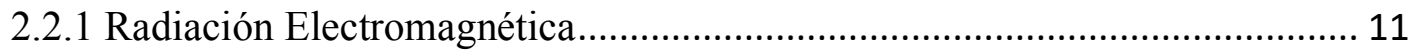

2.2.1.1 Función de Green ........................................................................ 13

2.3 Electromagnetismo Computacional ............................................................ 14

2.3.1 Clasificación de los métodos numéricos ............................................. 15

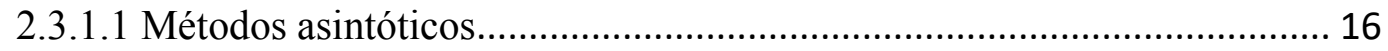

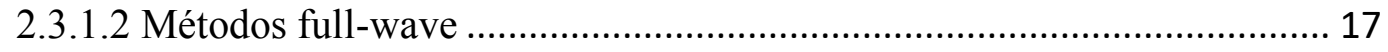

2.3.1.2.1 Método de los momentos............................................................... 18 
2.4 Software de simulación electromagnética comerciales........................................ 20

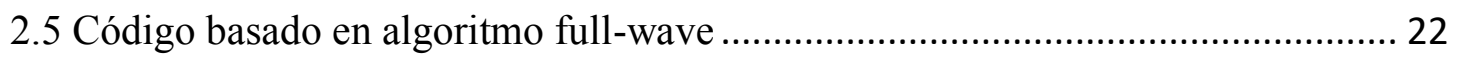

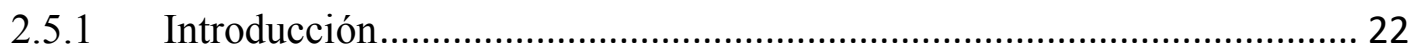

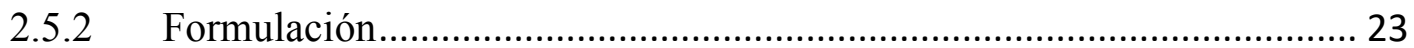

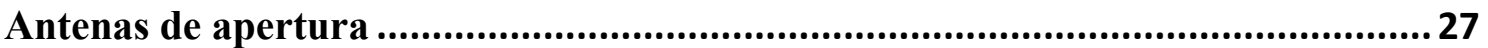

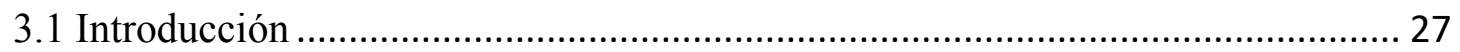

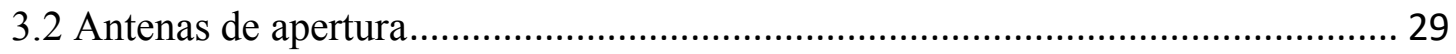

3.2.1 Parámetros básicos de antenas ...................................................................... 30

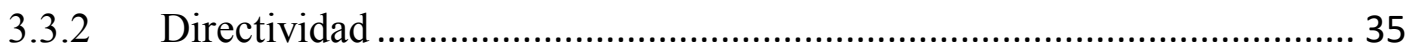

3.3.3 Eficiencia de apertura de antena ............................................................. 36

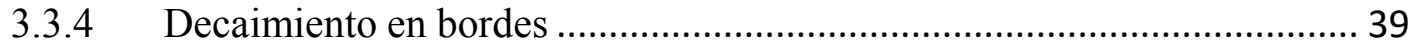

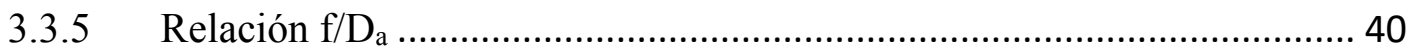

3.3.6 Perdidas por reflexión y transmisión................................................. 42

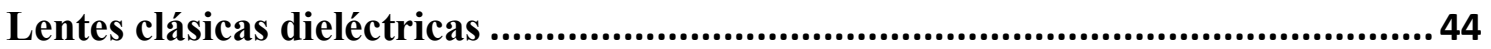

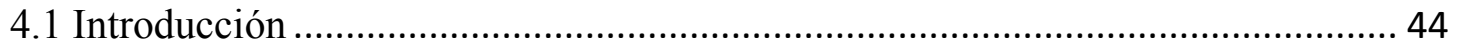

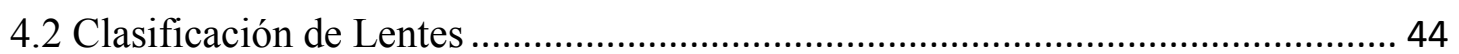

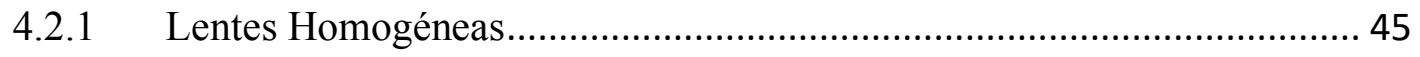

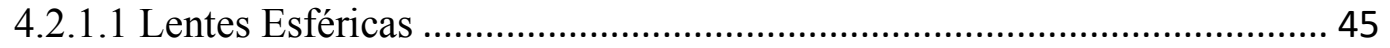

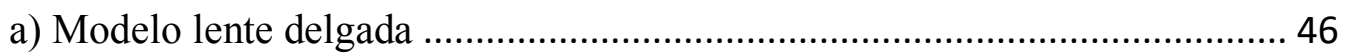

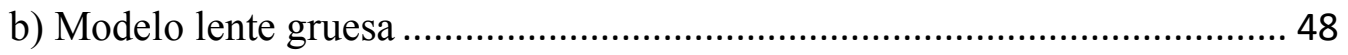

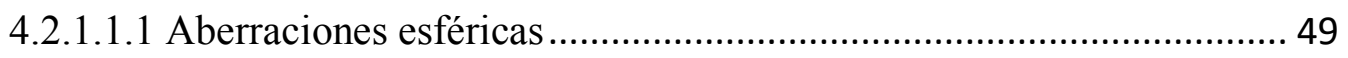

4.2.1.2 Lentes Asféricas ........................................................................ 50

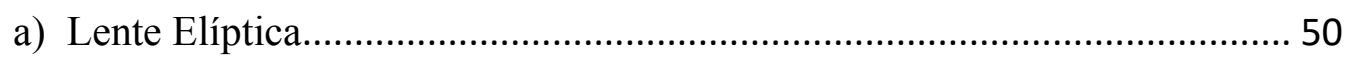

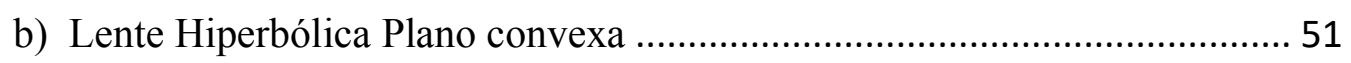

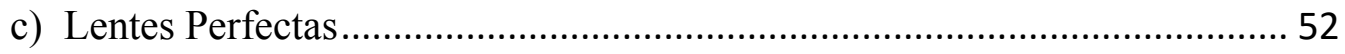

d) Lente escalonada de Fresnel.............................................................. 54

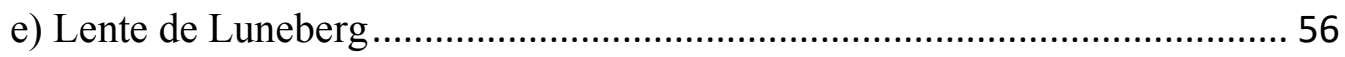


Diseño de antenas lente dieléctricas en Microondas mediante método full-wave .. 58

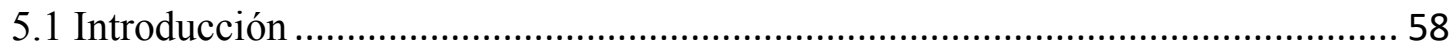

5.2 Parámetros de diseño de las antenas lente ............................................................. 59

5.2.1 Procedimiento para el diseño de antenas lentes ........................................... 60

5.3 Determinación del foco de una lente.................................................................. 61

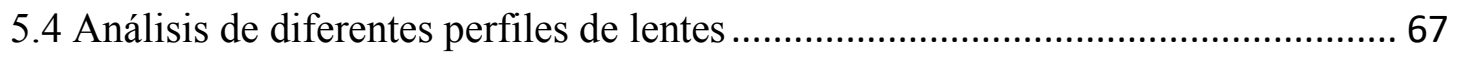

5.4.1 Simulaciones de lentes de perfiles cónicos ................................................67 67

5.4.2 Análisis de fase a la salida de una antena lente........................................... 70

5.4.3 Lente Escalonada de Fresnel .................................................................. 72

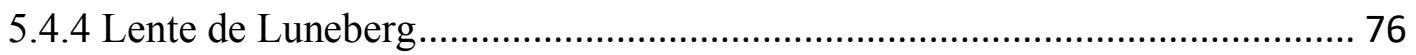

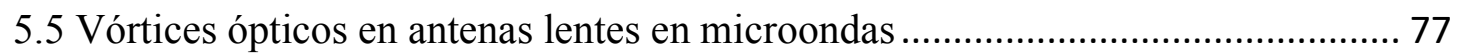

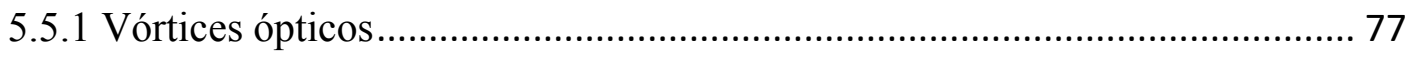

5.5.2 Simulaciones para evaluar vórtices ópticos en microondas ........................... 79

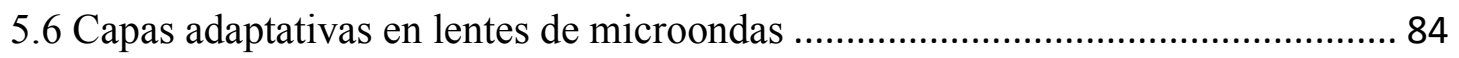

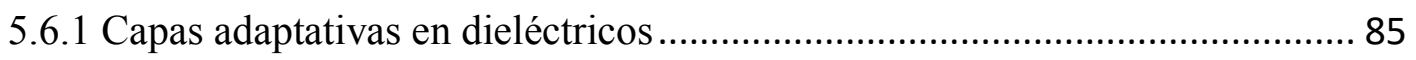

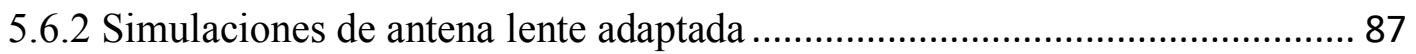

5.7 Simulaciones de lentes mediante software comercial ....................................... 98

5.7.1 Simulaciones full-wave mediante el software comercial Grasp .................. 99

5.7.2 Simulaciones con técnicas asintóticas mediante el software comercial Grasp

101

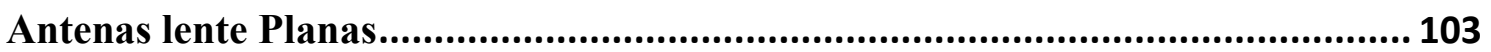

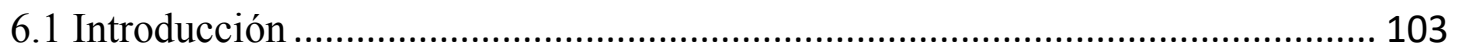

6.2 Lentes planas o superficies de cambio de fase................................................. 103

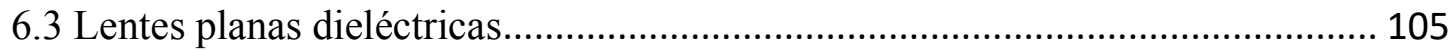

6.3.1 Diseño de una lente plana dieléctrica.................................................... 108

6.3.2 Simulaciones de antena lente plana dieléctrica ........................................ 110

6.3.3 Resultados de antena lente plana dieléctrica ........................................... 113

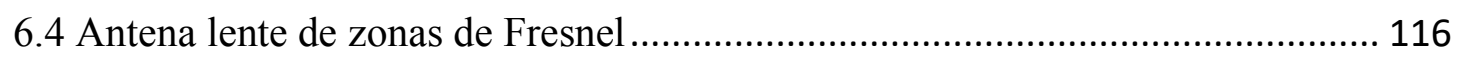

6.4.1 Diseño de lente de zonas de Fresnel....................................................... 117

6.4.2 Simulaciones de antena lente de zonas de Fresnel .................................... 118 
6.4.3 Resultados de antena lente de zonas de Fresnel 123

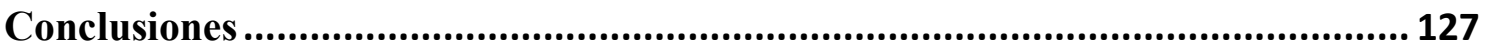

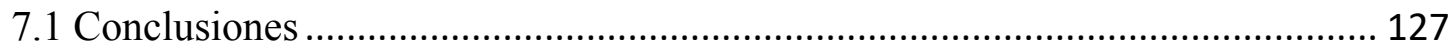

7.1.1 Diseño de antenas lente dieléctricas en Microondas mediante método full-

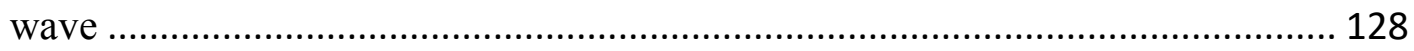

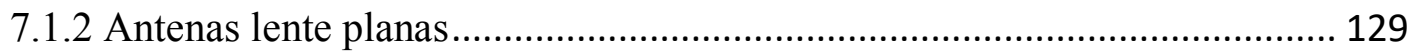

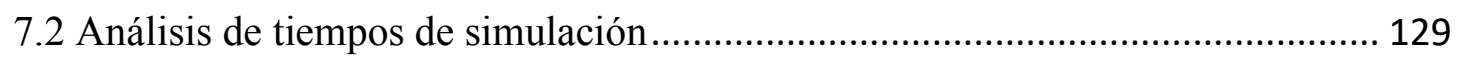

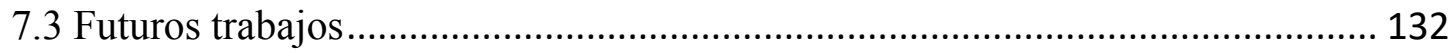

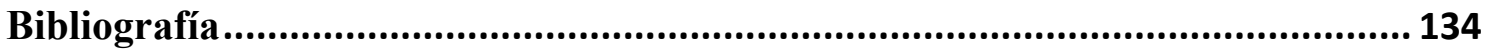




\section{Índice de Figuras}

Figura 2.1: Diferentes métodos de electromagnetismo computacional (CEM).......... 15

Figura 2.2: Métodos de simulación utilizados por el software Feko [51] .................. 20

Figura 2.3: Software de simulación Grasp............................................................ 21

Figura 3.1: Diagrama de funcionamiento de una antena lente en a) transmisión y b)

recepción.

28

Figura 3.2: Diagramas de antenas de apertura para el caso de a) Reflector y b) Antena

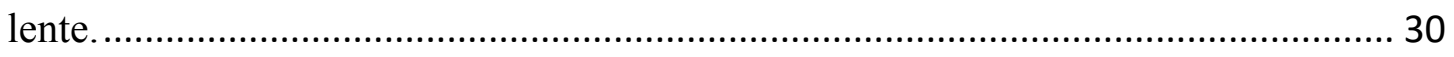

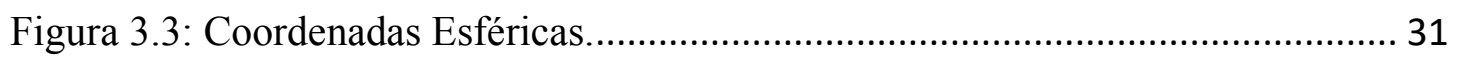

Figura 3.4: Radiación de Radiofuente en coordenadas Esféricas. ............................ 32

Figura 3.5: a) Diagrama en 3D, b) Diagrama Polar, c) Diagrama Cartesiano............ 34

Figura 3.6: Diagramas de radiación en 3D. ........................................................... 35

Figura 3.7: Lóbulo principal de radiación............................................................... 36

Figura 3.8: a) Baja eficiencia de Spillover b) Baja eficiencia de iluminación............. 38

Figura 3.9: a) Relación f/ $\mathrm{D}_{\mathrm{a}}$ Alto b) Relación $\mathrm{f} / \mathrm{D}_{\mathrm{a}}$ baja. .......................................... 41

Figura 3.10: Variación de los parámetros de eficiencia en función del ángulo $\beta \ldots . . . .42$

Figura 4.1: Clasificación de Lentes......................................................................... 45

Figura 4.2: Diseño de lente esférica biconvexa delgada.......................................... 46

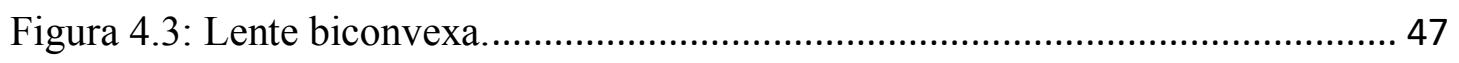

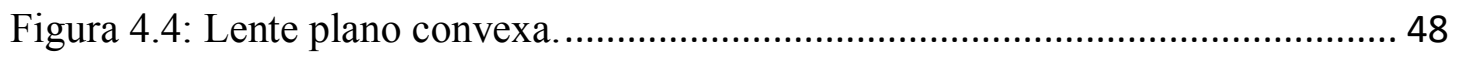

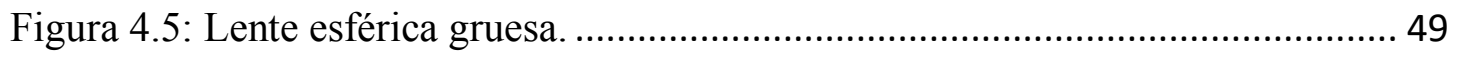

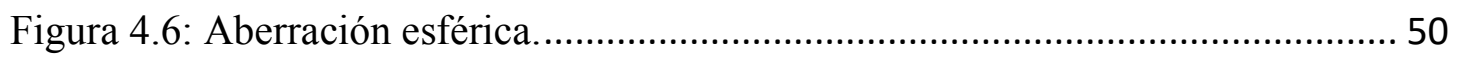

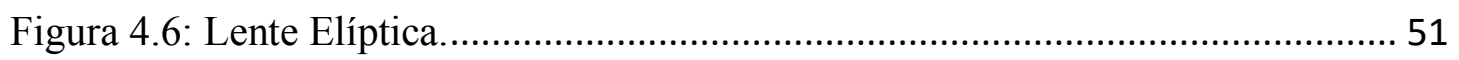

Figura 4.7: Lente Hiperbólica plano convexa...................................................... 52

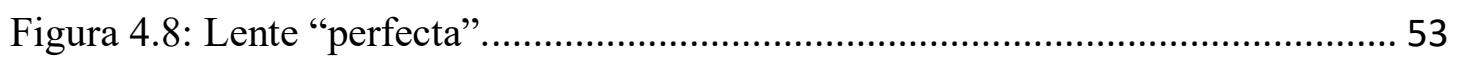

Figura 4.9: Diseño de lente escalonada de Fresnel. ................................................. 55

Figura 4.10: Lente escalonada de Fresnel............................................................... 55

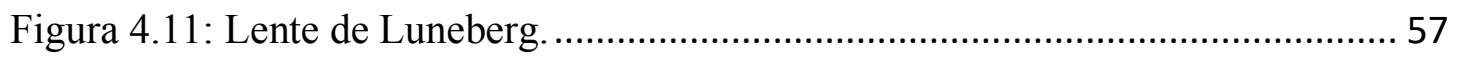

Figura 5.1: Proceso de diseño de antenas lente....................................................... 59

Figura 5.2: Esquema de simulación para determinación de la distancia focal de la

lente a) Hiperbólica y b) Esférica biconvexa............................................................ 62

Figura 5.3: Módulo del campo eléctrico cercano simulado para la antena lente a)

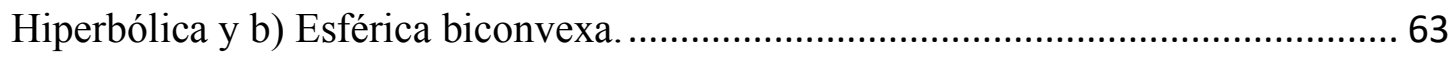

Figura 5.4: Módulo del campo eléctrico cercano simulado en el eje de la lente a)

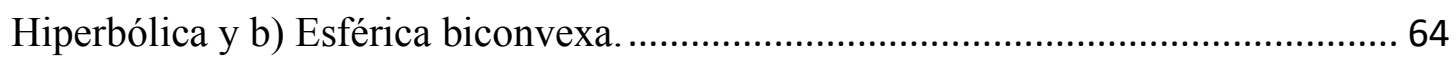

Figura 5.5: a) Modulo y b) Fase del campo eléctrico cercano para la lente elíptica... 66 
Figura 5.6: Módulo del campo eléctrico en transmisión de a) antena lente hiperbólica y b) antena lente elíptica. 68

Figura 5.7: Diagrama de radicación de a) antena lente hiperbólica y b) antena lente elíptica.

Figura 5.8: Módulo del campo eléctrico cercano a) dentro y fuera de la lente y b) dentro de la lente.

Figura 5.9: Fase del campo eléctrico dentro y fuera de la lente y fase del campo en la apertura.

Figura 5.10: Módulo del campo eléctrico cercano de la lente de zonas de Fresnel.... 74

Figura 5.11: Ganancia en función de la frecuencia para la antena lente de zonas de Fresnel.

Figura 5.12: Diagrama de radiación de la antena lente de zonas de Fresnel. 75

a)

b) 77

Figura 5.13: Lente de Luneberg. 77

Figura 5.14: Vórtices ópticos de primer orden para una carga topológica de $\mathrm{m}=1 \mathrm{y} \mathrm{m}$ $=5$ respectivamente.

Figura 5.15: Intensidad relativa del campo dentro y fuera de la lente en el espacio de simulación (cuadrado unidad) para la lente base (a) y la lente modificada (b). 80

Figura 5.16: Fase del campo dentro y fuera de la lente. a) Lente base y b) Lente modificada.

Figura 5.17: Ampliación de la Fig. 4b) del sector donde se ubican las dislocaciones. 82 Figura 5.18: Diagrama polar de a) la lente base y b) la lente modificada, es decir con bordes planos.

Figura 5.19: Características eléctricas de 2 medios y la ondas incidentes, reflejadas y transmitidas.

Figura 5.20: Características eléctricas de tres medios y la ondas incidentes, reflejadas y transmitidas.

Figura 5.21: Lente Hiperbólica a) sin adaptador, b) con adaptador y c) doble adaptador.

Figura 5.22: Intensidad relativa del campo dentro y fuera de la lente a) sin adaptar y

b) con doble adaptador.

Figura 5.23: Módulo del campo eléctrico en el eje de la lente a) sin adaptar y con adaptador simple y b) sin adaptar y con adaptador doble. 91

Figura 5.24: Ampliación de la Figura 5.22 b) entre el alimentador y la lente. Figura 5.25: a) Diagrama de radiación de la antena lente adaptada con una capa y sin adaptar, b) diagrama de radiación de la antena lente adaptada con doble capa y sin adaptar. .

Figura 5.26: Intensidad relativa del campo dentro y fuera de la lente elíptica a) sin adaptar y b) con doble adaptador. 95 
Figura 5.27: Módulo del campo eléctrico en el eje de la lente sin adaptar y adaptada.

$\ldots$

Figura 5.28: Diagrama de radiación de la lente sin adaptar y adaptada..................... 97

Figura 5.29: Antena lente diseñada con software Grasp 10.6.0................................. 99

Figura 5.30: Vista 3D de la antena lente simulada. .............................................. 100

Figura 5.31: Resultado del análisis BoR-MoM de la lente recubierta...................... 101

Figura 5.32: Resultado del análisis GO-PO de la lente no adaptada. ...................... 102

Figura 6.1: Esquema de antena a) lente dieléctrica biconvexa y b) lente plana. ...... 104

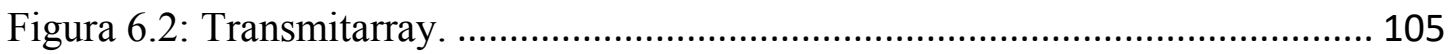

Figura 6.3: Antena lente plana dieléctrica y variación de la permitividad de la lente

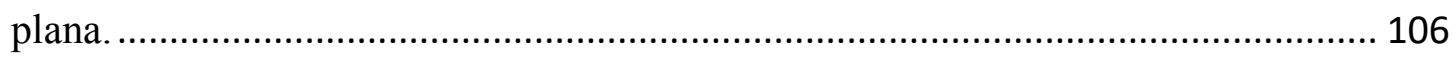

Figura 6.4: Cambio de fase a) Lente biconvexa y b) lente plana equivalente. ........ 106

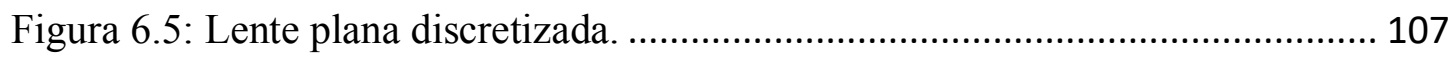

Figura 6.6: Lente plana con orificios............................................................... 107

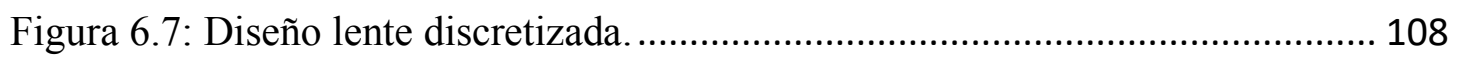

Figura 6.8: Enrejado a) triangular, b) cuadricular.............................................. 110

Figura 6.9: Modelo para la simulación de antena plana dieléctrica discretizada...... 111

Figura 6.10: Modelo simulado 3D..................................................................... 112

Figura 6.11: Ganancia de la antena lente simulada en $23 \mathrm{GHz}$.............................. 113

Figura 6.12: Prototipo de Lente plana con orificios............................................... 114

Figura 6.13: Medición de antena lente plana con orificios. .................................... 114

Figura 6.14: Ganancia simulada y medida para bocina y para antena lenta (bocina +

prototipo) en 23GHz. .................................................................................... 115

Figura 6.15: Fabricación de la antena lente plana dieléctrica. ................................. 116

Figura 6.16: Medición de la antena plana dieléctrica. .............................................. 116

Figura 6.17: Lente de zonas de Fresnel (FZP).................................................... 117

Figura 6.18: Esquema de lente de zonas de Fresnel. ............................................. 118

Figura 6.19: Diseño de lente de zonas de Fresnel................................................... 118

Figura 6.20: Diagrama de radiación simulado de a) la bocina sola y b) la bocina con la lente de zonas de Fresnel. ............................................................................... 119

Figura 6.21: Diagrama de radiación de la bocina sola y la antena lente................... 120

Figura 6.22: Diagrama de radiación de la antena lente para diferentes posiciones del

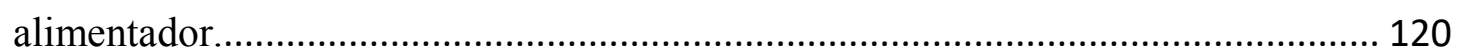

Figura 6.23: Diferentes diseños de la lente de zonas de Fresnel.............................. 121

Figura 6.24: Diagrama de radiación simulado de los diferentes diseños de la lente de

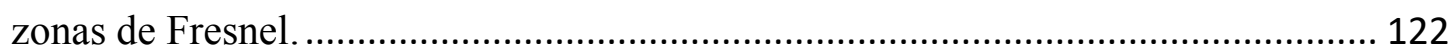

Figura 6.25: Antena lente de zonas de Fresnel Construida..................................... 123

Figura 6.26: Simulación 3D de la antena lente de zonas de Fresnel Construida...... 124 
Figura 6.27: Diagrama de radiación simulado para diferentes desplazamientos del alimentador.. 124

Figura 6.28: Diagrama cartesiano de campo lejano de la antena lente simulada...... 125 Figura 6.29: Diagrama de radiación medido del prototipo de la antena de zonas de Fresnel. 125 


\section{Índice de Tablas}

Tabla 5.1: Características de las antenas lentes diseñadas........................................ 62

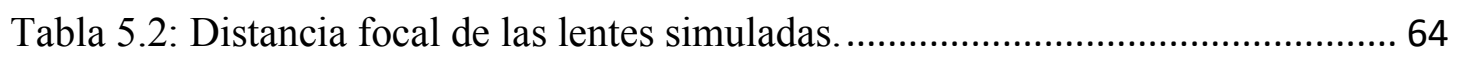

Tabla 5.3: Características eléctricas de las antenas lente Hiperbólica y elíptica. ....... 70

Tabla 5.4: Características de la lente hiperbólica como base de la lente escalonada de

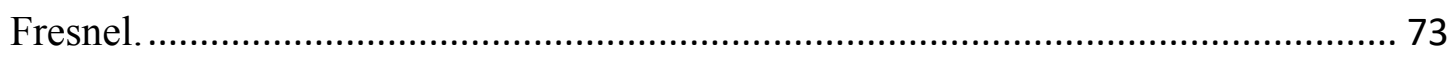

Tabla 5.5: Características eléctricas de la antena lente de zonas de Fresnel para 3

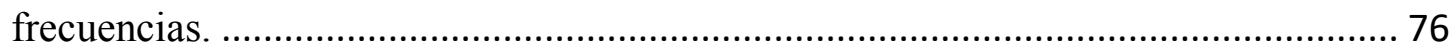

Tabla 5.6: Directividad máxima y ROE para los tres casos estudiados..................... 94

Tabla 5.7: Comparación de las características de radiación y ROE de las antenas lente de perfil Hiperbólico y Elíptico. ................................................................................ 97

Tabla 6.1: Constante dieléctrica por zonas. .......................................................... 111

Tabla 7.1: Características de la PC utilizada en las simulaciones. ........................... 129

Tabla 7.2: Tiempos de simulación de todos los problemas simulados. .................... 130 


\section{Nomenclaturas}

F $\quad$ Foco

$f \quad$ Distancia focal.

D Diámetro de la antena.

$f / D \quad$ Relación distancia focal a diámetro.

$(r, \Theta, \varphi) \quad$ Coordenadas esféricas.

E Campo eléctrico.

D Densidad de flujo eléctrico.

H Campo magnético.

B Densidad de flujo magnético.

$\rho \quad$ Densidad de cargas.

$J \quad$ Densidad de conducción de corriente.

$\mu \quad$ Permeabilidad eléctrica.

$\varepsilon \quad$ Permitividad eléctrica.

$Z_{i} \quad$ Impedancia intrínseca del medio.

$S_{a} \quad$ Densidad de potencia.

$F(\Theta, \varphi) \quad$ Intensidad de radiación o diagrama de radiación.

$\Omega_{p} \quad$ Ángulo sólido del diagrama de radiación.

$\Omega_{M} \quad$ Ángulo sólido del haz principal.

$\Omega_{m} \quad$ Ángulo sólido de lóbulos laterales.

$\Omega_{S} \quad$ Ángulo sólido de la radiofuente.

$\eta_{M} \quad$ Eficiencia del haz principal.

$\eta_{m} \quad$ Eficiencia de los lóbulos laterales

$\alpha_{1 / 2} \quad$ Ancho de haz o ancho de potencia mitad.

$\alpha_{\text {null }} \quad$ Ancho entre primer nulos. 
$D(\Theta, \varphi) \quad$ Directividad.

$\eta_{a} \quad$ Eficiencia de apertura.

$\eta_{i} \quad$ Eficiencia de iluminación.

$\eta_{s} \quad$ Eficiencia de spillover o sobreiluminación.

$\eta_{c} \quad$ Eficiencia de construcción o alineación.

$G(\Theta, \varphi) \quad$ Ganancia de antena.

$\varphi \quad$ Ángulos azimutales del haz principal de la antena.

$\Theta \quad$ Ángulos de elevación del haz principal de la antena.

$\lambda \quad$ Longitud de onda de radiación. 


\section{Resumen}

El estudio de las antenas lente si bien no resulta novedoso, ha recibido un fuerte impulso producto de las nuevas tecnologías en estas últimas décadas. El uso de nuevos materiales y técnicas ha permitido implementar antenas lente en distintas aplicaciones de telecomunicaciones en la banda de microondas.

Con el incremento de las capacidades computacionales actuales se ha hecho posible la simulación de antenas lente y ello ha permitido la optimización de sus diseños. Cuando las lentes son utilizadas en aplicaciones ópticas, éstas son estudiadas mediante técnicas asintóticas tales como el trazado de rayos, debido a que el tamaño de la lente es mucho mayor a la longitud de onda de la fuente. A frecuencias tales como las microondas otras técnicas deben ser aplicadas para lograr buenos resultados. En esta banda de frecuencias donde los resultados de la física óptica no son tan precisos, es fundamental contar con el aporte de datos y diseños obtenidos mediante software de simulación electromagnética basados en métodos full-wave.

La mayoría de los softwares de simulación electromagnética comerciales actuales que emplean métodos full-wave, cuando se ejecutan en computadoras personales estándares, no permiten simular lentes de gran diámetro en relación a la longitud de onda aplicada. Sin embargo, los sistemas antenas lente, al requerir una importante ganancia, necesitan lentes de gran diámetro, lo cual implica que muchas veces los softwares comerciales no sean aptos para simularlas.

El aporte de este trabajo de investigación es el estudio y el planteo de bases teóricas para el diseño y desarrollo de antenas lente para aplicaciones en la banda de microondas.

Para el diseño de lentes dieléctricas se aplica un novedoso código de simulación electromagnética que utiliza un método full-wave y permite simular lentes de gran tamaño con gran exactitud y en reducidos tiempos de cómputo comparado con los softwares de simulación comerciales.

Asimismo, se presentan diseños de antenas lente de vanguardia y resultados de mediciones de prototipos. 


\section{CAPÍTULO 1}

\section{Introducción}

\subsection{Antecedentes y motivación}

Lograr transmitir la información de manera confiable a grandes distancias se ha constituido, actualmente, en una de las más desafiantes demandas para aplicaciones de telecomunicaciones a frecuencias de microondas, tales como: enlaces punto-punto y punto-multipunto, enlaces satelitales, radares en banda $\mathrm{W}$, sistemas de comunicaciones en automóviles y trenes, etc. [1]. Es por ello que, contar con antenas de alta directividad resulta un requerimiento fundamental. Dentro de las antenas de apertura, los reflectores parabólicos, así como las lentes, son diseños típicos que permiten mejorar la directividad, siendo esta última directamente proporcional a su tamaño. Mientras la utilización de antenas parabólicas ha alcanzado gran desarrollo en aplicación de microondas, esto no ocurre con las antenas lente.

Las propiedades de las lentes han sido ampliamente estudiadas en el campo de la Óptica Geométrica, donde han encontrado su mayor uso [2]. En Óptica, la longitud de onda de las ondas electromagnéticas involucradas (luz visible) es mucho menor al tamaño de las lentes utilizadas, lo cual ha permitido el empleo de una teoría que plantea un modelo muy sencillo de la luz para su estudio, que es la Óptica Geométrica, la cual parte de las leyes fenomenológicas de Snell de la reflexión y la refracción.

La Óptica Geométrica usa la noción de trazado de rayos, que es una aproximación del comportamiento que corresponde a las ondas electromagnéticas cuando los objetos involucrados son de tamaño mucho mayor que la longitud de onda usada; ello permite despreciar los efectos derivados de la difracción, comportamiento ligado a la naturaleza ondulatoria de la luz.

Cuando las lentes son utilizadas en aplicaciones en la banda de las microondas, donde el tamaño de las lentes es comparable con la longitud de onda, no es posible aplicar la Óptica Geométrica sin perder exactitud. Consecuentemente, se debe recurrir a otros métodos conocidos como métodos de onda completa (métodos "full-wave") [3].

Por otro lado, las soluciones analíticas en problemas de electromagnetismo son solo posibles para algunas geometrías muy particulares, llamadas canónicas, en las que las condiciones de contorno se aplican a superficies con alguna coordenada constante en un sistema de coordenadas en el que la ecuación de onda sea separable. Además, es requisito, para obtener la solución analítica, que el medio comprendido entre estas 
superficies sea homogéneo. Ejemplos típicos son las soluciones modales para guías de onda rectangular o cilíndrica y la difracción de una onda incidente por objetos cilíndricos o esféricos.

Como las antenas en general no responden a este tipo de geometría, no es posible una solución analítica exacta y es necesario recurrir a la solución numérica del problema. Dichas soluciones, debido a la gran cantidad de cómputo requerido, son sólo susceptibles de obtención mediante simulación electromagnética.

Recién en los últimos años, gracias a los cada vez más potentes softwares de simulación electromagnética, es posible simular diferentes problemas de electromagnetismo. Para aplicaciones en la banda de microondas, tales como las antenas lente, se requiere de simuladores que utilicen métodos full-wave. El mayor inconveniente que presentan estos métodos, particularmente en las configuraciones de gran tamaño (en relación a la longitud de onda), es la elevada capacidad de cómputo, así como de tiempos de simulación requeridos.

Hoy en día existe un renovado interés en el estudio de las lentes dieléctricas en aplicaciones para ondas milimétricas. Esto se debe a que, para esta banda del espectro, las dimensiones de las lentes son tales que es viable su fabricación e implementación. Asimismo, se encuentran disponibles materiales dieléctricos de muy bajas pérdidas; y la utilización de máquinas de control numérico permite la fabricación de lentes sofisticadas con buenas tolerancias y a bajo costo.

Una de las principales tendencias en la actualidad en el campo de las antenas lente es el diseño de las denominadas "lentes planas" o "superficies de cambio de fase". Dichos diseños permiten reducir significativamente el espesor de las lentes y, por consiguiente, su tamaño y peso.

Esta tesis propone el estudio de antenas lente en microondas utilizando distintos métodos de simulación.

Para el diseño de antenas lente dieléctricas se utiliza un novedoso código de simulación basado en un método full-wave, que resuelve problemas de scattering electromagnético en tiempos mucho menores y para lentes de diámetros mayores que los softwares de simulación electromagnética comerciales actuales [4]. Este código de simulación full-wave permite determinar características de las antenas lentes dieléctricas y visualizar fenómenos electromagnéticos no vistos con los softwares electromagnéticos tradicionales. Asimismo, en esta tesis se utilizan softwares de simulación electromagnética comerciales para diseñar y simular antenas lente planas empleando materiales especiales. A fin de evaluar los diseños se construyen prototipos utilizando las más recientes técnicas y se analizan sus resultados y características. 


\subsection{Estado del Arte}

La más antigua referencia histórica al aumento de vista se remonta a los jeroglíficos egipcios del siglo $\mathrm{V}$ a.C. los cuales representaban lentes simples de vidrio. El registro escrito más antiguo del aumento de vista data del siglo I d. C. cuando Séneca, un tutor del emperador Nerón de Roma, escribió: «Letras, sin embargo, pequeñas y borrosas, son vistas más amplia y claramente a través de un globo o vaso lleno de agua» [5]. Nerón también dijo haber visto los juegos de gladiadores usando una esmeralda como lente correctora [6]. El uso de una lente convexa para una imagen más amplia es discutido en el libro de óptica de Alhacén (1021).

La ciencia de la óptica geométrica fue fundada en el siglo XVII. A las leyes de reflexión, conocidas desde Euclides, René Descartes y Willebrord Snell sumaron las leyes de refracción; Robert Boyle y Robert Hooke descubrieron la interferencia; Olaf Römer descubrió la velocidad finita de luz; F. M. Grimaldi y Hooke descubrieron el fenómeno de difracción y Newton descubrió la dispersión.

Se enunciaron dos teorías físicas de la luz en el siglo XVII. Christian Huygens formuló la teoría "ondulatoria" de la luz y Newton formuló una teoría de propagación de partículas. Huygens pensó a la luz como un movimiento longitudinal del éter cuya perturbación se extienden a una velocidad finita desde una fuente puntual. La posición más alejada de la luz en el espacio forma una superficie que él llamó frente de onda. En un medio inhomogéneo esta superficie es una esfera. Huygens supuso que cuando la perturbación llega a cualquier punto del éter dicho punto imparte su movimiento a los puntos vecinos.

El uso de lentes dieléctricas como parte de una antena es casi tan antiguo como la demostración de ondas electromagnéticas de Hertz. De hecho, en 1888, Oliver Lodge usó una lente dieléctrica en sus experimentos para ondas de $1 \mathrm{~m}$ de longitud de onda [7]. Sin embargo, no fue hasta la Segunda Guerra Mundial que la investigación sobre antenas lente avanzó considerablemente.

En 1944, R. k. Luneberg en sus apuntes de la Brown University sobre "Teoría Matemática de la Óptica" [8] considera los medios generales en los cuales el índice de refracción $\mathrm{n}$ es una función continua de $\rho$ (distancia desde un punto fijo), y es independiente de las consideraciones angulares. La lente de Luneberg es una lente esférica que tiene la propiedad de concentrar los rayos de una onda plana incidente en un punto de su superficie. Como la esfera tiene simetría de revolución, la propiedad se cumple con independencia de la dirección de incidencia.

En 1946 W. E. Kock [9] diseña un lente con guías paralelas. En vez de reducir la velocidad de fase con un material dieléctrico propone utilizar planos conductores paralelos en forma de guías de onda. Para esa configuración el índice de refracción efectivo de la lente resulta menor a uno. 
En la década del $50 \mathrm{~A}$. N. Whitehead en su trabajo "The theory of parallel-plate media for Microwave Lenses" [10] analiza la reflexión y refracción de lentes de placas planas paralelas. Posteriormente J.E. Eaton [11] analiza la lente de Luneberg mediante ecuaciones integrales y por su parte G. D. Peeler [12] diseña una lente de Luneberg 2D mediante 2 placas rellenas de Poliestireno.

En la década del 60 K. Kay en "Electromagnetic Theory and Geometrical Optics" repasa la ya estudiada relación entre el Electromagnetismo y la Óptica Geométrica (GO, por sus siglas en inglés) y la aplica a casos prácticos [13].

La síntesis de lentes basadas en Óptica Geométrica se ha utilizado en la literatura para diferentes especificaciones de diseño que van desde problemas simples de corrección de fase [14], hasta problemas de barrido de haz o haces múltiples [15], o problemas de conformación de haz [16-17]. Se ha aplicado para la síntesis de lentes con simetría de eje, así como para cualquier lente de forma arbitraria [18-21], para lentes de índice de refracción no uniforme [22] o, de hecho, para cualquier combinación de los casos anteriores.

Como se observa de la bibliografía, las antenas lente fueron investigadas ampliamente durante el desarrollo temprano de las antenas de microondas y dichas investigaciones fueron influenciadas por el extenso trabajo existente desde la óptica. Posteriormente, el interés disminuyó un poco a medida que las antenas lente fueron superadas por los reflectores para antenas de alta eficiencia y gran apertura

Más recientemente, a medida que el interés de la investigación se ha expandido hacia el uso de bandas de frecuencia de onda milimétrica y submilimétrica, las antenas lente han atraído nuevamente la atención de los desarrolladores Impulsados por el uso de software de simulación electromagnética. En 1996 Hongo K. analiza las características de radiación de una lente dieléctrica plano-convexa mediante simulación numérica [23].

Unos años más tarde, en 2011, se realizan comparaciones entre antenas lente dieléctricas de perfiles elípticos e hiperbólicos en aplicaciones de microondas basadas en simulaciones con software de uso comercial [24].

Una de las mayores tendencias en los últimos años es al estudio de las lentes planas o superficies de cambio de fase. Conjuntamente al diseño de nuevos materiales, dichas lentes resultan de menor tamaño que las lentes dieléctricas y consecuentemente tienen menor peso. En 2015 y 2016 se publicaron trabajos con antenas lente planas de Luneberg para aplicaciones inalámbricas en microondas [25-26]. Un diseño novedoso e interesante publicado en 2016 es una antena lente plana dieléctrica no homogénea construida a partir de orificios en un sustrato [27].

Otro tipo de antena lente plana es la denominada lente de zonas de Fresnel (FZP, por sus siglas en inglés), la cual utiliza elementos conductores. Si bien su principio de 
funcionamiento fue estudiado algunos años atrás [28-30], actualmente se están desarrollando nuevos diseños, debido a los nuevos métodos de fabricación [31-33].

El uso de nuevas aplicaciones en las frecuencias de los Terahertz también ha impulsado al estudio de antenas lente [34-38]. El potencial de esta banda ha sido probado por numerosos tipos de aplicaciones que incluyen imágenes médicas, pruebas no destructivas, observación espacial y espectroscopia, gracias a su buen compromiso entre la resolución espacial y penetración [39].

Las superficies selectivas de frecuencia (FSS) también se han utilizado para diseñar lentes planas en microondas. El estudio y diseño de superficies selectivas en frecuencia también han resurgido los últimos años gracias a los cada vez más potentes softwares de simulación electromagnética y las nuevas técnicas de fabricación [40-45].

Recientemente se publicaron libros sobre antenas lente dieléctricas modernas [4647] donde se presentan los principios de diseño y análisis principales de antenas lente conocidos hasta la fecha.

El avance de la tecnología y la importancia que han tomado las antenas lente para aplicaciones en microondas en la última década, han promovido la realización de la presente Tesis, donde se profundiza en el estudio e investigación en esta área buscando nuevos métodos de simulación y desarrollo de nuevos materiales con el fin de lograr un avance en sus problemáticas.

\subsection{Objetivos de la Tesis}

\subsubsection{Objetivos Generales}

Investigación y desarrollo de antenas lente mediante técnicas de simulación para aplicaciones en microondas.

\subsubsection{Objetivos Específicos}

Los objetivos específicos de la presente Tesis son:

- Estudiar e investigar los sistemas de antenas lente y sus características.

- Analizar e implementar diferentes softwares de simulación electromagnética disponibles y sus diversas técnicas aplicados a antenas lente.

- Analizar un algoritmo matemático que resuelva problemas de electromagnetismo de antenas lentes de gran tamaño por métodos full-wave.

- Estudiar los diferentes perfiles de lentes y analizar su impacto en los sistemas antenas lente. 
- Investigar métodos de diseño óptimo para antenas lente dieléctricas utilizando nuevos métodos de simulación.

- Estudiar y desarrollar lentes planas y superficies de cambio de fase.

\subsection{Esquema de la Tesis}

La tesis está organizada en 7 capítulos los cuales abordan varios aspectos del análisis, el diseño y la validación experimental de los conceptos de antena propuestos. A continuación, se muestra un breve resumen sobre los contenidos de cada capítulo.

En el presente Capítulo se repasa el estado del arte de las antenas lentes en microondas y se describe la problemática de su diseño, así como la motivación y principales objetivos de esta Tesis.

En el Capítulo 2 se describen los fundamentos de radiación electromagnética y las diferentes técnicas de simulación electromagnética. Se repasan los principales métodos que utilizan los softwares de simulación electromagnética y se presenta el fundamento de un novedoso código de simulación electromagnética no comercial basado en técnicas full-wave.

Las características eléctricas de las antenas lentes se estudian en el Capítulo 3. Dado que las antenas lente se consideran antenas de apertura, se exponen las características eléctricas de las antenas de aperturas tales como ganancia, eficiencia, etc.

En el Capítulo 4 se repasan las ecuaciones de diseño de lentes clásicas y se analizan diferentes perfiles de lentes cónicas y otras lentes clásicas dieléctricas tales como la lente escalonada de Fresnel.

El Capítulo 5 se enfoca el proceso de diseño de antenas lente dieléctricas en microondas por medio de simulación electromagnética. Se emplea el código de simulación estudiado en el Capítulo 2 el cual permite observar fenómenos electromagnéticos en antenas lentes en microondas no observables con otros simuladores electromagnéticos. Se presentan resultados novedosos arrojados por las simulaciones.

En el Capítulo 6 se estudian las denominadas lentes planas o superficies de cambio de fase. Se analizan dos diseños: la antena lente plana dieléctrica y la lente de zonas de Fresnel. Se simulan y se validan experimentalmente los resultados de mediciones para prototipos construidos.

En el Capítulo 7 se describen las conclusiones de esta Tesis Doctoral y se presentan propuestas para trabajos futuros. 


\subsection{Publicaciones}

Algunos de los resultados de este trabajo de Tesis han dado origen a una serie de artículos que se han presentado en congresos y jornadas nacionales e internacionales, los cuales se listan a continuación:

\section{Congresos Internacionales:}

-P. M. Gross, J. Vernieri, F. Vico, A. Bava, M. F. Bataller. “Análisis en frecuencia de lente de Fresnel en microondas mediante simulación Full-Wave”. XXXIII Simposium Nacional de la Unión Científica Internacional de Radio (URSI 2018). 5 a 7 de septiembre de 2018. Granada, España.

- P. M. Gross, J. Vernieri, F. Vico, A. Bava, M. F. Bataller. “Lens Antennas focus determination using Full-Wave simulation". 12th European Conference on Antennas and Propagation (Eucap2018). 9 al 13 de Abril de 2018. Londres, Reino Unido.

- P. M. Gross, J. Vernieri, F. Vico, A. Bava, M. F. Bataller, J. R. Robert. “Aplicación de nuevo código de simulación para estudio de lentes en microondas en 2D". XXXII Simposium Nacional de la Unión Científica Internacional de Radio (URSI 2017). 6 a 8 de Septiembre de 2017. Cartagena, España.

\section{Jornadas y Congresos Nacionales:}

- P. M. Gross, J. Vernieri, F. Vico, A. Bava, M. F. Bataller. "Estudio de adaptación en antenas lente dieléctricas mediante simulación full-wave". XVIII Reunión de trabajo en Procesamiento de la Información y Control (RPIC 2019). 18 a 20 de septiembre de 2019. Bahía Blanca, Argentina.

- P. M. Gross, J. P. Ciafardini, J. Vernieri, A. Bava. "Antena lente plana dieléctrica". X Congreso Argentino de Tecnología Espacial. 10-12 Abril, 2019. Ciudad autónoma de Buenos Aires, Argentina.

-P. M. Gross, J. Vernieri, G. Rodríguez. "Simulaciones Full-Wave en la banda de microondas". 5tas Jornadas de Investigación y Transferencia. 9 al 11 de Abril 2019. Facultad de Ingeniería. UNLP. La Plata, Argentina.

- P. M. Gross, J. Vernieri, F. Vico, A. Bava, M. F. Bataller. “Análisis de las características de antenas lentes en microondas mediante método full-wave". IEEE Argencon 2018. 6 al 8 de junio de 2018. San Miguel de Tucumán. Tucumán. Argentina.

- P. M. Gross, J. Vernieri, F. Vico, A. Bava, M. F. Bataller. "Simulación de antenas lente en microondas". 102 ${ }^{\mathrm{a}}$ Reunión Nacional de la Asociación Física Argentina. 26 al 29 de Septiembre de 2017. La Plata, Buenos Aires, Argentina. 
- P. M. Gross, J. Vernieri, F. Vico, A. Bava, M. F. Bataller. "Estudio de vórtices ópticos en antenas lente". XVII Reunión de trabajo en Procesamiento de la Información y Control (RPIC 2017). 20 a 22 de septiembre de 2017. Mar del Plata, Argentina.

- P. M. Gross, J. P. Ciafardini, F. Vico, A. Bava, M. F. Bataller. "Antenas lente con metamateriales y su aplicación en tecnología espacial". IX Congreso Argentino de Tecnología Espacial. 26-28 abril, 2017. Córdoba, Argentina. 


\section{CAPÍTULO 2}

\section{Electromagnetismo y simulación electromagnética}

\subsection{Introducción}

Antes de que se desarrollara la computadora digital el análisis y diseño de dispositivos y estructuras electromagnéticas eran en gran parte experimentales. Una vez que aparecieron los lenguajes computacionales y numéricos, éstos comenzaron a ser usados de inmediato para abordar problemas electromagnéticos que no podían resolverse analíticamente. Este campo es conocido como el electromagnetismo computacional (CEM, por sus siglas en ingles). Se han desarrollado numerosas técnicas de análisis numérico de gran alcance en esta área en los últimos 50 años. A medida que el poder de cómputo de las computadoras crece, también se profundiza en los algoritmos aplicados, así como la complejidad y el tamaño de los problemas que pueden resolverse mediante ellos.

Si bien los datos que se obtienen de las mediciones experimentales son invaluables, el proceso de fabricación, montaje y medición puede ser muy costoso, por lo que contar con simuladores puede resultar de gran ahorro. La ventaja que implica poder simular el comportamiento de los dispositivos y sistemas antes de que se construyan ha sido el impulsor fundamental del desarrollo de algoritmos electromagnéticos computacionales confiables. De esta forma es posible lograr niveles de optimización en los diseños que serían dificultosos o incluso imposibles si se hicieran de forma experimental. El electromagnetismo computacional también ayuda a proporcionar información fundamental sobre problemas electromagnéticos a través del poder de la computación y visualización por computadora, lo que la convierte en una de las áreas más importantes de la ingeniería de hoy [48].

En el presente Capítulo se introducen las ecuaciones fundamentales del electromagnetismo, conocidas como ecuaciones de Maxwell, y las ecuaciones generales que permiten resolver problemas electromagnéticos. La solución de las ecuaciones de Maxwell es compleja y, para problemas reales, es necesario recurrir a aproximaciones. Como se mencionó previamente la aproximación numérica a las ecuaciones de Maxwell es la rama conocida como Electromagnetismo computacional. Por este motivo se repasan los conceptos básicos del electromagnetismo computacional y los diferentes métodos numéricos que se aplican. 
Asimismo, los softwares comerciales de simulación electromagnética y los métodos numéricos que éstos utilizan son aplicables bajo ciertas hipótesis, presentando ciertas limitaciones bajo determinadas condiciones. Como se expondrá a lo largo del presente Capítulo, un ejemplo de ello son las antenas lente en microondas de gran tamaño.

Por lo tanto, es necesario recurrir a nuevas herramientas de simulación. Debido a ello, para el diseño de lentes dieléctricas se utilizó un novedoso algoritmo que permite resolver algunas de las limitaciones de los softwares comerciales [4]. El algoritmo fue implementado en forma de código computacional en MATLAB y, para hacer referencia a dicho código, en la presente Tesis se lo denomina código full-wave. En este Capítulo se exponen los fundamentos del mismo.

\subsection{Electromagnetismo}

Se puede definir el Electromagnetismo como el estudio de los campos eléctricos y magnéticos y su interacción.

Fue James Clark Maxwell (1831-1879), uno de los físicos más brillantes del siglo XIX, quien combinó el trabajo de sus predecesores en una forma teórica elegante y postuló que los campos eléctricos variables deberían generar campos magnéticos; luego mostró que ello implica movimiento ondulatorio. Asimismo, Hermann Ludwig Ferdinand Helmholtz (1821-1894) fue uno de los primeros en reconocer el significado de las predicciones de Maxwell al respecto; en 1888, su alumno Heinrich Rudolph Hertz (1857-1894) demostró experimentalmente que los campos electromagnéticos de hecho se propagan, y lo hacen a la velocidad de la luz.

Las siguientes cuatro ecuaciones de Maxwell se consideran la base de todos los fenómenos eléctricos y magnéticos. El núcleo del electromagnetismo moderno puede ser descripto por dichas ecuaciones que, en su forma diferencial resultan:

$$
\begin{gathered}
\nabla \times \overrightarrow{\mathrm{E}}=-\mathrm{M}-\frac{d}{d t} \overrightarrow{\mathrm{B}} \\
\nabla \times \overrightarrow{\mathrm{H}}=\overrightarrow{\mathrm{J}}+\frac{d}{d t} \overrightarrow{\mathrm{D}} \\
\nabla \cdot \overrightarrow{\mathrm{D}}=q_{e} \\
\nabla \cdot \overrightarrow{\mathrm{B}}=q_{m}
\end{gathered}
$$

con las ecuaciones constitutivas asociadas 


$$
\begin{aligned}
& \overrightarrow{\mathrm{B}}=\mu \overrightarrow{\mathrm{H}} \\
& \overrightarrow{\mathrm{D}}=\varepsilon \overrightarrow{\mathrm{E}}
\end{aligned}
$$

donde $\overrightarrow{\mathrm{E}}$ es el campo eléctrico, $\overrightarrow{\mathrm{D}}$ es la densidad de flujo eléctrico, $\overrightarrow{\mathrm{B}}$ es la densidad de flujo magnético, $\overrightarrow{\mathrm{H}}$ es la intensidad de campo magnético, $\overrightarrow{\mathrm{J}}$ es la densidad de corriente, $\mu$ es la permeabilidad magnética del medio, $\varepsilon$ es la permitividad eléctrica del medio, $M$ es una corriente magnética y $\mathrm{q}_{\mathrm{e}} \mathrm{y} \mathrm{q}_{\mathrm{m}}$ son cargas eléctricas y magnéticas respectivamente.

Si bien la corriente magnética $\mathrm{M}$ y la carga $\mathrm{q}_{\mathrm{m}}$ no son cantidades físicamente realizables, se utilizan frecuentemente como herramientas matemáticas para problemas de radiación y dispersión electromagnética [49].

\subsubsection{Radiación Electromagnética}

Los problemas de radiación electromagnética implican obtener los campos en todas partes del espacio debido a un conjunto de corrientes eléctricas y magnéticas. Los problemas de dispersión (scaterring) pueden ser considerados como problemas de radiación donde las corrientes locales son generadas por otro conjunto de corrientes o un campo incidente. Combinando las ecuaciones (2.1 y 2.2) en una región donde solo existen corrientes eléctricas $\boldsymbol{J}$ y cargas $\boldsymbol{q}_{\boldsymbol{e}}\left(\boldsymbol{M}=\boldsymbol{q}_{\boldsymbol{m}}=\boldsymbol{0}\right)$ se obtiene

$$
\nabla x \nabla x \boldsymbol{E}=-j \omega \mu \nabla x \boldsymbol{H}=\omega^{2} \mu \varepsilon \boldsymbol{E}-j \omega \mu \boldsymbol{J}
$$

Utilizando la identidad vectorial

$$
\nabla x \nabla x \boldsymbol{E}=\nabla(\nabla \cdot \boldsymbol{E})-\nabla^{2} \boldsymbol{E}
$$

permite escribir (2.7) de la forma

$$
\nabla(\nabla \cdot \boldsymbol{E})-\nabla^{2} \boldsymbol{E}-k^{2} \boldsymbol{E}=-j \omega \mu \boldsymbol{J}
$$

donde $k$ es el número de onda, $k=\omega \sqrt{\mu \varepsilon}=2 \pi / \lambda$. Sustituyendo (2.3) en la ecuación anterior se obtiene

$$
\nabla^{2} \boldsymbol{E}-k^{2} \boldsymbol{E}=j \omega \mu \boldsymbol{J}+\frac{\nabla \boldsymbol{q}_{\boldsymbol{e}}}{\varepsilon}
$$


Aplicando la ecuación de continuidad

$$
\nabla \cdot \boldsymbol{J}=-j \omega \boldsymbol{q}_{\boldsymbol{e}}
$$

se obtiene

$$
\nabla^{2} \boldsymbol{E}-k^{2} \boldsymbol{E}=j \omega \mu \boldsymbol{J}-\frac{1}{j \omega \varepsilon} \nabla(\nabla \cdot \boldsymbol{J})
$$

Dado que las ecuaciones de Maxwell son lineales, se puede considerar que $\mathrm{J}$ es una superposición de fuentes puntuales distribuidas en un dado volumen. Por lo tanto, si se conoce la respuesta de una fuente puntual, se puede resolver el problema original integrando dicha respuesta sobre el volumen. Se puede hacer el uso de esta idea para convertir la ecuación (2.12) en una ecuación integral. Como dicha ecuación implica tres ecuaciones escalares separadas, se puede considerar una de ellas, por ejemplo

$$
\nabla^{2} \boldsymbol{E}_{\boldsymbol{x}}-k^{2} \boldsymbol{E}_{\boldsymbol{x}}=j \omega \mu\left(\boldsymbol{J}_{\boldsymbol{x}}+\frac{1}{k^{2}} \frac{\partial}{\partial \mathrm{x}}\left(\nabla \cdot \boldsymbol{J}_{\boldsymbol{x}}\right)\right)
$$

Introduciendo la función de Green $G\left(r, r^{\prime}\right)$ [Ver sección 2.2.1.1], que satisface la ecuación escalar de Helmoltz

$$
\nabla^{2} G\left(r, r^{\prime}\right)-k^{2} G\left(r, r^{\prime}\right)=-\delta\left(r, r^{\prime}\right)
$$

Asumiendo $G\left(r, r^{\prime}\right)$ conocida, se puede obtener $\boldsymbol{E}_{\boldsymbol{x}}$ mediante

$$
\boldsymbol{E}_{\boldsymbol{x}}(r)=-j \omega \mu \iiint_{V} G\left(r, r^{\prime}\right)\left[\boldsymbol{J}_{\boldsymbol{x}}\left(r^{\prime}\right)+\frac{1}{k^{2}} \frac{\partial}{\partial \mathrm{x}}\left(\nabla^{\prime} \cdot \boldsymbol{J}\left(r^{\prime}\right)\right)\right] d r^{\prime}
$$

Generalizando a la forma vectorial

$$
\boldsymbol{E}(r)=-j \omega \mu \iiint_{V} G\left(r, r^{\prime}\right)\left[\boldsymbol{J}\left(r^{\prime}\right)+\frac{1}{k^{2}}\left(\nabla^{\prime} \nabla^{\prime} \cdot \boldsymbol{J}\left(r^{\prime}\right)\right)\right] d r^{\prime}
$$

donde integral se realiza sobre el volumen de $\boldsymbol{J}$. De la misma manera se puede obtener el campo magnético

$$
\boldsymbol{H}(r)=-j \omega \varepsilon \iiint_{V} G\left(r, r^{\prime}\right)\left[\boldsymbol{M}\left(r^{\prime}\right)+\frac{1}{k^{2}}\left(\nabla \nabla^{\prime} \cdot \boldsymbol{M}\left(r^{\prime}\right)\right)\right] d r^{\prime}
$$


Para utilizar (2.16) y (2.17), se debe encontrar la solución a la ecuación (2.14) y obtener $G\left(r, r^{\prime}\right)$.

\subsubsection{Función de Green}

La función de Green es una función matemática usada como núcleo de un operador lineal integral y permite la resolución de ecuaciones diferenciales no homogéneas con condiciones de contorno especificadas.

Una función de Green $G(x, s)$, de un operador diferencial $L$, en un punto $\mathrm{S}$, es cualquier solución a la ecuación

$$
L(G(x, s))=\delta(s-x)
$$

donde $\delta$ es la función delta de Dirac. Las propiedades de las funciones de Green pueden utilizarse para resolver problemas del tipo

$$
\operatorname{Lu}(x)=f(x)
$$

donde $\mathrm{f}$ es una función conocida, $\mathrm{y} u$ es una función a determinar.

En términos generales, si tal función $G$ puede ser encontrada para el operador $L$, entonces, si multiplicamos la ecuación (2.18) por la función de Green por $f(s)$, y luego lo integramos con respecto a $\mathrm{s}$, obtenemos

$$
\int L G(x, s) f(s) d s=\int \delta(x-s) f(s) d s=f(x)
$$

Relacionando con la ecuación (2.19)

$$
L u(x)=\int L G(x, s) f(s) d s
$$

Como $L$ es un operador lineal y solo actúa en $\mathrm{x}$

$$
L u(x)=L \int G(x, s) f(s) d s
$$

Por lo tanto 


$$
u(x)=\int G(x, s) f(s) d s
$$

Esto indica que se puede obtener la función $u(x)$ mediante el conocimiento de la función de Green asociada al operador $L$, resolviendo la integral del lado derecho de la ecuación (2.23). Este proceso se basa en la linealidad del operador $L$. No todos los operadores $L$ admite función de Green.

Las funciones de Green que permiten resolver la ecuación de Helmholtz en 2 y 3 dimensiones están dadas por las ecuaciones 2.24 y 2.25 respectivamente [48].

$$
\begin{gathered}
g_{2 D}(r)=\frac{1}{4 i} H_{0}(k r) \\
g_{3 D}(r)=\frac{1}{4 \pi} \frac{e^{i k r}}{r}
\end{gathered}
$$

$H_{0}$ denota la función de Hankel de orden cero de primer tipo.

\subsection{Electromagnetismo Computacional}

Electromagnetismo computacional es el procedimiento que se debe seguir para modelar y simular el comportamiento de los campos electromagnéticos en dispositivos o alrededor de estructuras.

Por lo general, soluciones analíticas exactas solo son posibles para una pequeña cantidad de casos donde la geometría que define el problema es simple, llamadas canónicas. La utilización de técnicas numéricas ofrece la posibilidad de resolver potencialmente cualquier problema electromagnético.

El electromagnetismo computacional consiste en utilizar técnicas numéricas para resolver las ecuaciones de Maxwell en vez de obtener soluciones analíticas.

Las principales ventajas de utilizar el electromagnetismo computacional es que permite obtener datos a través de software sin necesidad de tener que construir dispositivos y realizar medidas, lo cual podría resultar altamente costoso. Con la aparición de las computadoras y la disminución del costo de cómputo, los métodos numéricos se han convertido en una herramienta fundamental para la resolución de problemas de electromagnetismo.

Existen diversos métodos numéricos que permiten resolver problemas electromagnéticos los cuales se describen en las próximas secciones. 


\subsubsection{Clasificación de los métodos numéricos}

Existen diversas formas de clasificar los métodos numéricos [3, 48]. Los principales métodos de electromagnetismo computacional se muestran en la Fig. 2.1.

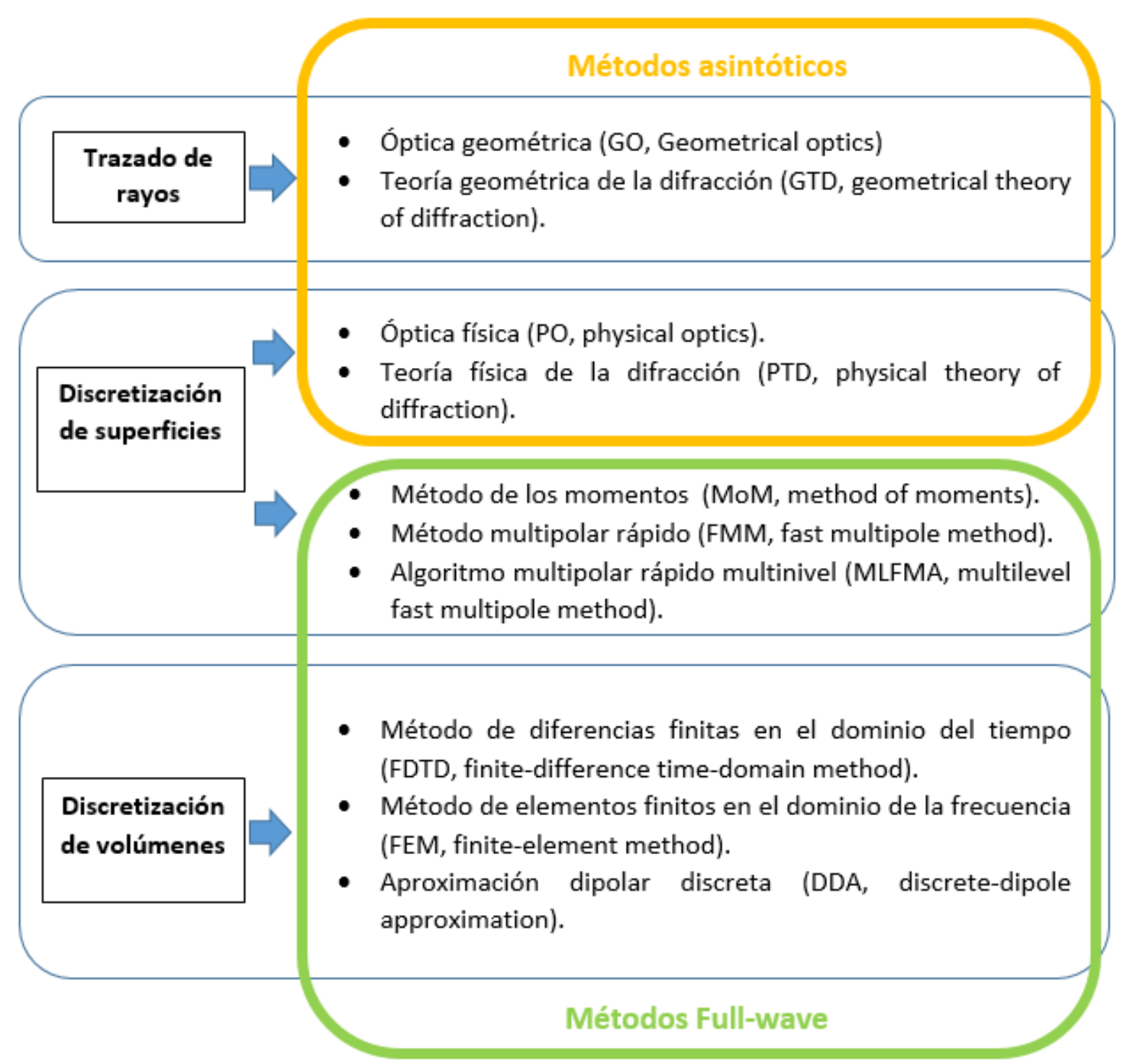

Figura 2.1: Diferentes métodos de electromagnetismo computacional (CEM).

Según el modelo geométrico que usan sus formulaciones para caracterizar los dispersores, los métodos se pueden clasificar en tres tipos: de trazado de rayos, de discretizaciones de superficies y de discretizaciones de volúmenes.

Otra clasificación tiene en cuenta la precisión de los resultados y el ámbito de aplicación, es decir, el tipo de problemas en los que cada método es aplicable. Los métodos se pueden clasificar en dos grandes grupos: métodos de onda completa (fullwave) y métodos asintóticos, también llamados de alta frecuencia. 
Como se expresó en el Capítulo 1, el objetivo de esta Tesis es el estudio de antenas lente en microondas mediante simulación electromagnética. Para ello es fundamental comprender los diferentes tipos de métodos numéricos que se aplican a dichos casos y sus limitaciones en dicha aplicación.

En los próximos incisos se exponen las principales diferencias entre los métodos asintóticos y los métodos full-wave.

\subsubsection{Métodos asintóticos}

Los métodos asintóticos requieren aproximaciones fundamentales en las ecuaciones de Maxwell, cuya validez aumenta asintóticamente con la frecuencia. La precisión es mejor cuanto mayores, en comparación con la longitud de onda, sean el tamaño y los radios locales de curvatura de los objetos dispersores. Es decir, estos métodos resultan convenientes cuando las dimensiones de las estructuras son mucho mayores respecto a la longitud de onda.

Si se utilizan correctamente estos métodos son muy potentes, pero las aproximaciones subyacentes de la física limitan su uso para problemas generales. Además, a diferencia de los métodos full-wave donde la ley de Moore y el aumento resultante en la velocidad de la computadora y la memoria extienden continuamente los límites de la aplicabilidad, los métodos asintóticos tienen límites fundamentales. A pesar de estos inconvenientes, los métodos asintóticos son ampliamente utilizados en problemas donde proporcionan una precisión aceptable y los métodos full-wave no son aplicables por cuestiones de costes computacionales, por ejemplo, en problemas de cálculos de coberturas de telefonía móvil o de Wi-fi en entornos rurales o urbanos [49].

Dentro de los métodos asintóticos se considera como método superficial basado en ecuaciones integrales de superficie a la óptica física (PO, Physical Optics) puesto que esta está basada en discretizaciones de superficies, empleando aproximaciones válidas solo para objetos eléctricamente grandes. La PO admite un método de corrección para incluir difracción, llamado teoría física de la difracción (PTD, Physical Theory of Diffraction), aunque dicha corrección solamente es aplicable en medios conductores eléctricos perfectos (PEC, Perfect Electric Conductors).

Finalmente, los métodos basados en trazado de rayos como la óptica geométrica (GO, Geometrical Optics), la cual es posible complementar con la teoría geométrica de la difracción (GTD, Geometrical Theory of Diffraction), tienen un nivel de exactitud menor que el resto, puesto que su teoría asociada solo considera unos pocos términos dominantes en una solución asintótica de alta frecuencia de las ecuaciones de Maxwell, aunque son muy versátiles en simulaciones con múltiples reflexiones. 


\subsubsection{Métodos full-wave}

Estos métodos resultan convenientes cuando las dimensiones de las estructuras son comprables con la longitud de onda.

Los métodos full-wave son potencialmente muy precisos [3]. Central para todos estos métodos es la idea de discretizar una propiedad electromagnética desconocida (incertidumbre), por lo general la corriente de superficie para el método MoM, y el campo eléctrico para los métodos FEM y FDTD (para este último el campo $\mathrm{H}$ también se discretiza). Este proceso de discretización es también conocido como mallado (meshing) y consiste en dividir la geometría en un gran número de pequeños “elementos". Estos últimos pueden ser segmentos unidimensionales, superficies (generalmente triángulos) o elementos tridimensionales, dependiendo del problema a resolver y el método a utilizar.

Por cada elemento, se asume una dependencia funcional para la variación espacial de la incertidumbre, por ejemplo, una aproximación lineal, donde la amplitud (y posiblemente la fase) de la incertidumbre está determinada por la aplicación del método al conjunto de elementos que aproximan la geometría original. Esta dependencia funcional se denomina función base (o expansión).

La precisión de los métodos full-wave está relacionada con la discretización (por ejemplo, el tamaño del mallado). Mientras más fino es el mallado (elementos más pequeños, aumentando así la resolución geométrica), mejor es la precisión de los métodos. Sin embargo, la cantidad de elementos está limitada por la capacidad computacional disponible. Para el electromagnetismo en radio frecuencia los requerimientos del mallado están determinados para poder calcular la fase adecuadamente. Por muchos años, la comunidad científica ha estado trabajando una regla de 10 segmentos por longitud de onda. Este criterio se originó a partir de problemas con antenas. Sin embargo, el conocimiento de los métodos y la experiencia permiten aplicar otros criterios.

Los métodos basados en discretizaciones volumétricas, como el método de diferencias finitas en el dominio del tiempo (FDTD, Finite-Difference Time-Domain) y el método de elementos finitos en el dominio frecuencial (FEM: Finite-Element Method), tienen las ventajas de que permiten modelar fácilmente medios no homogéneos, y sus formulaciones matemáticas 3D asociadas son relativamente simples. Sin embargo, adolecen del hecho de que el sistema de ecuaciones lineales resultantes posee un número de incógnitas proporcional al volumen simulado, por lo que la demanda computacional crece muy rápidamente al aumentar las dimensiones eléctricas consideradas en la simulación. Los métodos basados en discretizaciones de superficies presentan características que los hacen computacionalmente más eficientes que los volumétricos.

Las formulaciones empleadas en los métodos superficiales están basadas en ecuaciones integrales de superficie (SIE, Surface Integral Equations) que, a diferencia de las formulaciones volumétricas, son matemáticamente más difíciles de implementar en 
un código computacional, en parte debido a los diversos tipos de singularidades de la función de Green. Otra desventaja de este tipo de métodos es la imposibilidad de simular medios no homogéneos generales, aunque presentan la gran ventaja de que únicamente requieren discretizar las interfaces, es decir las superficies bidimensionales que delimitan los objetos dispersores. Esta discretización de superficies se realiza mediante facetas triangulares para las cuales los métodos calculan corrientes superficiales con las que finalmente se computan los campos dispersados.

Entre los métodos superficiales destacan el método de los momentos (MoM, Method-of-Moments) y sus optimizaciones computacionales. Dichas optimizaciones mejoran los tiempos de cálculo a cambio de introducir un error numérico controlable con respecto a los resultados del MoM puro. Entre ellas se destacan el método multipolar rápido (FMM, Fast Multipole Method) y algoritmo multipolar rápido multinivel (MLFMA, Multilevel Fast Multipole Algorithm).

Como se mencionó previamente, uno de los métodos full-wave más utilizados es el método de los momentos, o MoM [50].

Dado a que el código full-wave de simulación electromagnética que se aplica en esta tesis para simular lentes dieléctricas está basado en el método de los momentos, es conveniente repasar los conceptos fundamentales de dicho método.

\subsection{Método de los momentos}

La idea básica de dicho método consiste en reducir una ecuación funcional en una ecuación matricial, y luego resolver la ecuación matricial por medio de técnicas conocidas. El procedimiento general para resolver ecuaciones lineales, se denomina método de los momentos.

Consideremos la siguiente ecuación inhomogenea:

$$
L(f)=g
$$

donde $L$ es un operador lineal (integral, diferencial), $g$ es la fuente o excitación (función conocida), y $f$ es la incertidumbre (función a determinar). Dicha incertidumbre para problemas de electromagnetismo puede corresponder a cargas o a corrientes, mientras que la excitación puede ser el campo eléctrico. Si se expande $f$ en una suma de $\mathrm{N}$ funciones base ponderadas:

$$
f=\sum_{n=1}^{N} a_{n} f_{n}
$$


donde $a_{n}$ son los "pesos" de la función base $f_{n}$. Como el operador $L$ es lineal, sustituyendo (2.27) en (2.26) se obtiene:

$$
\sum_{n=1}^{N} a_{n} L\left(f_{n}\right) \approx g
$$

donde el residuo está dado por:

$$
R=g-\sum_{n=1}^{N} a_{n} f_{n}
$$

Las funciones base se eligen para modelar el comportamiento esperado de la función a determinar en todo su dominio, y pueden ser escalares o vectores dependiendo del problema. Si las funciones base tienen soporte local en el dominio, se llaman locales o funciones de base subseccional. Si su soporte abarca todo el dominio del problema, se llaman funciones base globales o de dominio completo.

Se puede generalizar el método considerando condiciones de frontera previamente impuestas. Se define el producto interno o momento entre una función base $f_{n}\left(r^{\prime}\right)$ y una función de prueba o peso $f_{m}(r)$ como

$$
<f_{n}, f_{m}>=\int_{f_{m}} f_{m}(r) \int_{f_{n}} f_{n}\left(r^{\prime}\right) d r^{\prime} d r
$$

donde las integrales son integrales de línea, superficie o volumen dependiendo de la base y funciones de prueba. Si se requiere que el producto interno de cada función de prueba con la función residual sea cero

$$
\sum_{n=1}^{N} a_{n}<f_{n}, L\left(f_{m}\right)>=<f_{m}, g>
$$

Lo cual arroja una ecuación matricial de $N x N$ de la forma $\boldsymbol{Z a}=\boldsymbol{b}$ con elementos de la matriz

$$
z_{m n}=<f_{m}, L\left(f_{n}\right)>
$$

Y los elementos del vector del lado derecho de (2.31)

$$
b_{m}=<f_{m}, g>
$$


En el método de los momentos, cada función de base interactúa con todas las demás mediante la función de Green y la matriz del sistema resulta completa. Todos los elementos de la matriz deben ser almacenados en la memoria.

Existen numerosas funciones de prueba y funciones base ampliamente detalladas en la bibliografía [50].

\subsection{Software de simulación electromagnética comerciales}

Los software de simulación comerciales utilizan diferentes métodos tanto en el dominio del tiempo como en el dominio de la frecuencia. Algunos métodos numéricos comúnmente utilizados son por ejemplo; métodos full-wave tales como MoM, FDTD, FEM y MLFMM y métodos asintóticos tales como PO, LE-PO, RL-GO, UTD. Asimismo, se utiliza hibridación de métodos para resolver problemas complejos $\mathrm{y}$ grandes en múltiples escalas, permitiendo acortar los tiempos de cómputo.

La Figura 2.2 muestra las técnicas de simulación recomendadas por el software comercial ALTAIR FEKO [51] en función del tamaño eléctrico de los objetos (relación de la longitud de onda aplicada con el tamaño) y su complejidad (relación de los radios de curvatura del objeto con la longitud de onda aplicada).

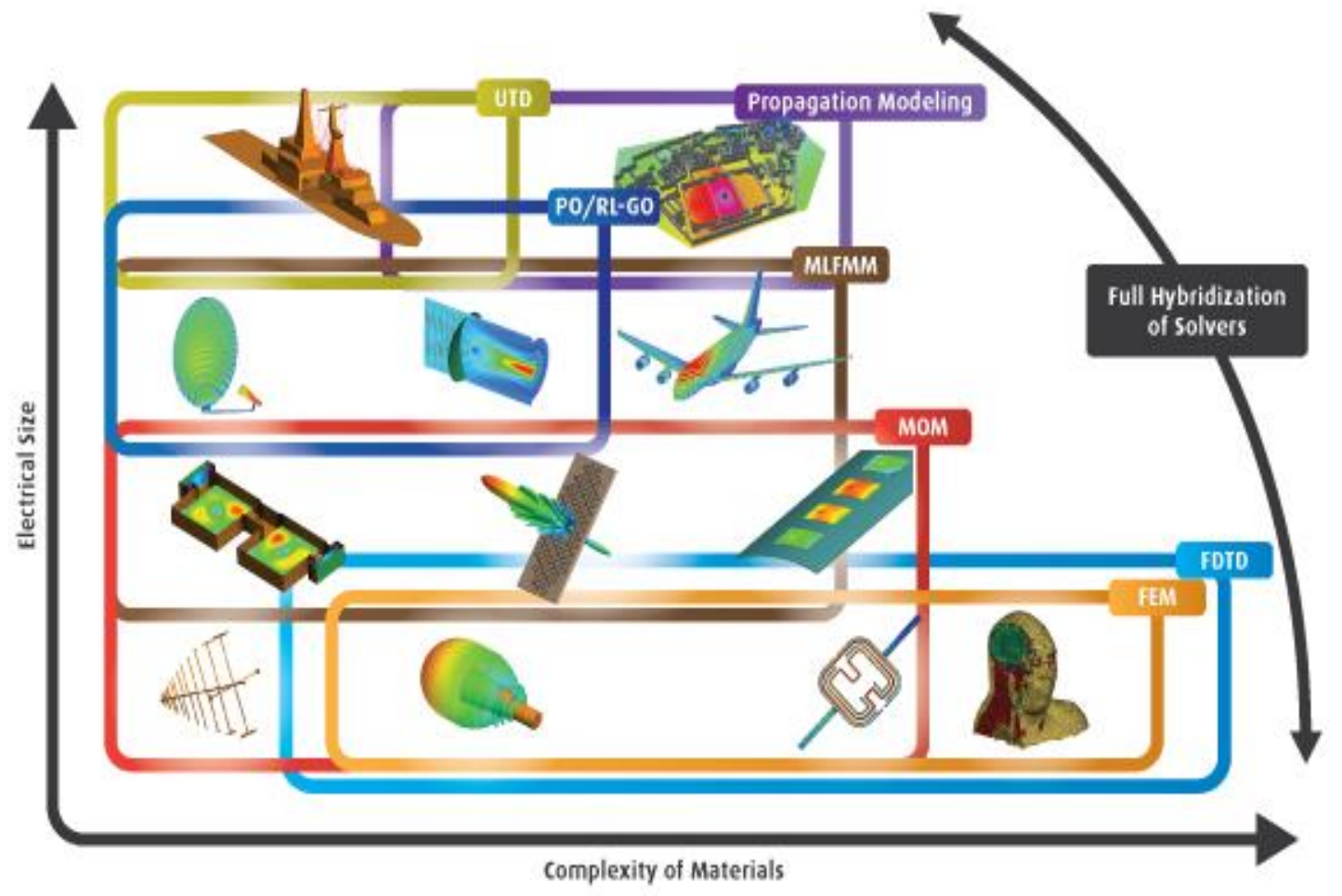

Figura 2.2: Métodos de simulación utilizados por el software Feko [51]. 
Como se observa de la Figura 2.2, a medida que el tamaño eléctrico de los objetos aumenta, se recomienda usar técnicas asintóticas tales como GO. El caso de lentes y reflectores es un ejemplo de ello.

Como se mencionó previamente, para lentes en microondas donde el tamaño de las lentes es eléctricamente grande pero no lo suficiente como para aplicar métodos asintóticos sin cometer errores, es fundamental contar con simulaciones full-wave.

El software comercial TICRA GRASP [52] permite simular lentes de gran tamaño por un método full-wave denominado Método de los Momentos para cuerpo de Revolución (BoR-MoM, por sus siglas en inglés). Dicho método permite simulaciones full-wave para cuerpos que tienen geometría de revolución [53-59].
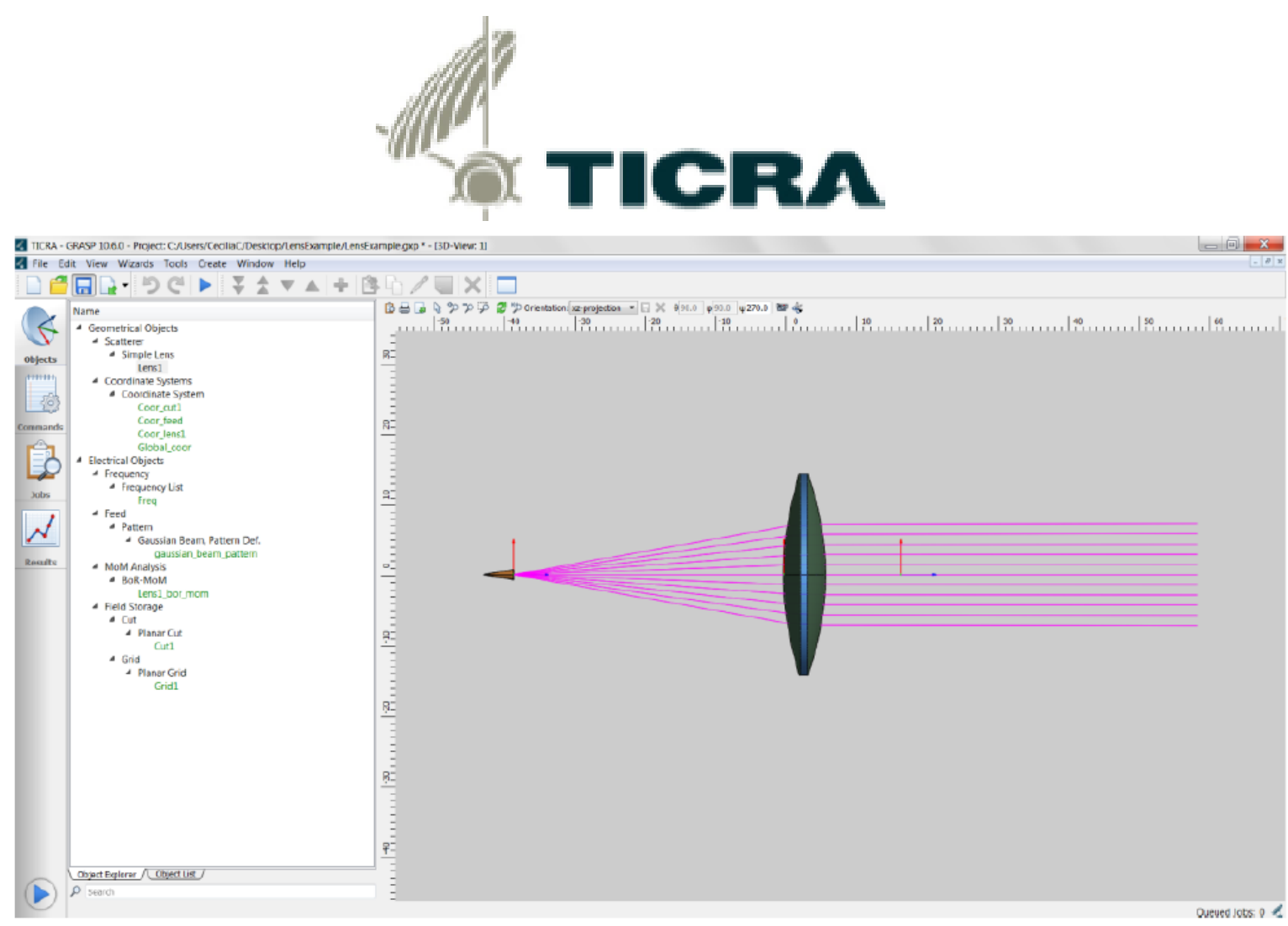

Figura 2.3: Software de simulación Grasp.

Es posible utilizar métodos full-wave aún para objetos de gran tamaño con software comerciales, pero eso requiere de gran capacidad computacional y elevados tiempos de simulación. La capacidad computacional necesaria, tanto desde el punto de vista de software como de hardware, muchas veces excede la capacidad de las computadoras personales estándares habituales en los hogares o laboratorios. Esta situación genera limitaciones para estudiar las antenas lente en microondas.

Con el fin de poder simular lentes dieléctricas para aplicaciones en microondas se desarrolló para esta Tesis un código de simulación electromagnética basado en un 
algoritmo matemático rápido, recientemente publicado que permite resolver problemas electromagnéticos sobre hardware de una computadora personal y bajar considerablemente los tiempos de cómputo respecto a la aplicación de un software comercial [4]. Asimismo, dicho código permite visualizar fenómenos electromagnéticos que no es posible observar mediante los softwares comerciales.

\subsection{Código basado en algoritmo full-wave}

\subsubsection{Introducción}

Parte de los diseños de antenas lente dieléctricas efectuados en la presente Tesis fueron realizados por un código basado en un algoritmo recientemente publicado [4], que en la presente Tesis se denomina Código full-wave. Una de las principales ventajas de este código es que utiliza un método full-wave y permite simular elementos dieléctricos tales como antenas lente dieléctricas de gran tamaño con alta precisión y con muy bajos tiempos de simulación. Dicho tipo de problemas son de muy difícil solución con software de simulación comerciales que utilicen métodos full-wave mediante computadores personales.

Este software permite diseñar cualquier tipo de objeto dieléctrico en $2 \mathrm{D}$ a través de una función contraste y utilizar diferentes perfiles de alimentadores de ondas electromagnéticas, obteniendo como resultado el módulo y la fase del campo y el diagrama polar de campo lejano en muy corto tiempo de cómputo.

En el código se introduce un algoritmo rápido para calcular potenciales volumétricos. El algoritmo se basa en regularizar la transformada de Fourier de la función de Green cortando la interacción en el espacio físico más allá del dominio de interés. Esto permite la aplicación directa de la cuadratura trapezoidal y la FFT estándar, con convergencia superalgebraica para datos suaves. Además, el método se puede interpretar como el empleo de una discretización Nystrom del operador integral correspondiente, con entradas de matriz que pueden obtenerse explícita y rápidamente. Esto es útil en el diseño de precondicionadores o solucionadores directos rápidos para una variedad de ecuaciones integrales de volumen. El método propuesto permite el cálculo de cualquier derivada del potencial, a costa de una FFT adicional.

El código fue desarrollado en MATLAB [60]. Los resultados se obtienen en un espacio cuadrado unidad de $\mathrm{N}$ por $\mathrm{N}$ nodos y con un largo y ancho en longitudes de onda variable, por lo que resulta adimensional con respecto a la frecuencia. La elección de $\mathrm{N}$ debe ir en concordancia con el tamaño de la celda unidad elegido para tener una buena precisión en los resultados. 


\subsubsection{Formulación}

Muchos problemas en computación científica requieren la solución de una ecuación diferencial parcial elíptica de coeficientes constantes sujeta a condiciones de contorno o radiación adecuadas. En muchos casos, la función de Green del espacio libre para la ecuación correspondiente es conocida, pero implica interacciones no locales (de largo alcance). Un ejemplo típico es la ecuación de Helmholtz en $\mathrm{R}^{\mathrm{d}}$.

$$
\Delta \varphi+k^{2} \varphi=f
$$

donde $\varphi$ puede ser pensado como un potencial acústico y $f$ una distribución conocida de fuentes acústicas, donde se supone está definida en el dominio acotado $D=$ $[-1 / 2,1 / 2]^{d}$. Esto se puede hacer sin pérdida de generalidad volviendo a escalar el parámetro k de la ecuación de Helmholtz. La solución de (2.34), que satisface la condición de radiación de Sommerfeld, está dada por:

$$
\varphi(x)=\int_{D} g_{k}(x-y) f(y) d y
$$

donde $g_{k}(r)=\frac{1}{4 i} H_{0}(k r)$ para $d=2$ y $g_{k}(r)=\frac{1}{4 \pi} \frac{e^{i k r}}{r}$ para $d=3$. Aquí $r=\|r\|_{2} \mathrm{y}$ $H_{0}$ denota la función de Hankel de orden cero de primer tipo.

Debe tenerse en cuenta que el núcleo de interacción es de largo alcance, requiriendo que los algoritmos rápidos sean prácticos y singulares en $r=0$, lo que requiere técnicas de cuadratura precisas. En algunas aplicaciones, una tercera dificultad es que la densidad de la fuente $f$ es altamente no homogénea, requiriendo discretización adaptativa. En tales configuraciones, se requieren métodos jerárquicos, intrínsecamente adaptativos. Sin embargo, cuando la densidad es uniforme y está bien resuelta mediante una malla uniforme, es más conveniente (y generalmente más eficiente) utilizar los métodos de Fourier. Esta última técnica es la utilizada por el código.

Existen dos formas diferentes para las cuales se puede aplicar el método de Fourier para el cálculo de (2.35). La primera es mediante la discretización directa de la ecuación con una regla trapezoidal corregida localmente. La suma neta toma la forma de una convolución discreta (aperiódica) y, por lo tanto, se puede calcular utilizando la FFT con relleno de ceros en $\mathrm{O}\left(\mathrm{N}^{2} \log \mathrm{N}\right)$ operaciones [4].

Alternativamente, usando el teorema de convolución puede escribirse

$$
\varphi(x)=\mathcal{F}^{-1}\left(\frac{F(s)}{|s|^{2}-k^{2}}\right)=\left(\frac{1}{2 \pi}\right)^{d} \int_{R^{d}} e^{i s x}\left(\frac{F(s)}{|s|^{2}-k^{2}}\right) d s
$$


Donde

$$
F(s)=\mathcal{F}(f)(s) \int_{D} e^{i s x} f(x) d x
$$

$\mathcal{F}$ denota la transformada de Fourier. El hecho de que $f(x)$ sea suave permite calcular la integral de Fourier en (2.37) con precisión "espectral". También garantiza que el error al truncar la integral de Fourier en la transformada inversa decae rápidamente con $|\mathbf{s}|$. La principal dificultad para emplear los métodos de Fourier es la singularidad $\frac{1}{|s|^{2}-k^{2}}$ en el integrando.

En el caso de la ecuación de Poisson, la singularidad se simplifica $\frac{1}{|s|^{2}}$.

En la ecuación de Poisson en tres dimensiones, por ejemplo, el cambio a coordenadas esféricas anula la singularidad por completo. Este enfoque se vuelve técnicamente más complicado para la ecuación de Helmholtz, donde la singularidad vive en la esfera $|\mathrm{s}|=\mathrm{k}$.

Resulta que hay un método simple que funciona para todas las funciones de Green de largo alcance, independientemente de la dimensión, que requiere solo la regla trapezoidal, alcanza la precisión espectral y es acelerado por la FFT estándar. Además, las entradas de la matriz que corresponden a este método de alto orden se calculan fácilmente, una característica útil para las estrategias de preacondicionamiento o los solucionadores directos cuando se usan métodos de volumen integral para resolver ecuaciones diferenciales parciales de coeficiente variable.

Si se restringe la solución de $\varphi(x)$ a una "caja" unidad $D \subset R^{d}$. Luego, la distancia máxima entre cualquier fuente y punto destino en $\mathrm{D}$ es $\sqrt{d}$. Se define

$$
g_{k}^{L}(r)= \begin{cases}\frac{1}{4 \pi} H_{0}(k r) \operatorname{rect}\left(\frac{r}{2 L}\right) & \text { si } d=2 \\ \frac{1}{4 \pi} \frac{e^{i k r}}{r} \operatorname{rect}\left(\frac{r}{2 L}\right) & \text { si } d=3\end{cases}
$$

con $\operatorname{rect}(x)$ definida para ser un intervalo unitario

$$
\operatorname{rect}(x)=\left\{\begin{array}{l}
1 \text { para }|x|<\frac{1}{2} \\
0 \text { para }|x|>\frac{1}{2}
\end{array}\right.
$$

Si se fija $L>\sqrt{d}$ en $d$ dimensiones entonces la solución a (2.35) es indistinguible a 


$$
\varphi(x)=\int_{D} g_{k}^{L}(x-y) f(y) d y
$$

Dado que $g_{k}^{L}$ tiene soporte compacto, el teorema de Paley-Wiener implica que su transformada de Fourier $G_{k}^{L}$ es entera. Además, es fácil de calcular [4]. En el caso del operador de Laplace en tres dimensiones

$$
G_{k}^{L}=2\left(\frac{\sin \left(\frac{L s}{2}\right)}{s}\right)^{2}
$$

Así, la ecuación de Poisson en tres dimensiones tiene la solución

$$
\varphi(x)=\frac{2}{(2 \pi)^{3}} \int_{R^{3}} e^{i s x}\left(\frac{\sin \left(\frac{L|s|}{2}\right)}{|s|}\right)^{2} F(s) d s
$$

La discretización por la regla trapezoidal en el dominio $\left[-\frac{N}{2}, \frac{N}{2}\right]^{d}$ permite realizar una evaluación rápida utilizando nada más que la FFT.

En particular el código desarrollado permite resolver la siguiente ecuación de derivadas parciales:

$$
\Delta \varphi+\mathrm{k}^{2}(1+\mathrm{q}(\mathrm{x}, \mathrm{y})) \varphi=0
$$

donde $\varphi=\varphi_{\text {inc }}+\varphi_{\text {Scat }}$ y la ecuación integral utilizada es la de LipmannSchwinger:

$$
-\rho+\mathrm{k}^{2} \mathrm{qV} \text { k }(\rho)=\mathrm{k}^{2} \mathrm{q} \varphi_{\text {inc }}
$$

y la correspondiente representación de la solución:

$$
\varphi_{\text {Scat }}=\mathrm{V}_{\mathrm{k}}(\rho)
$$

Debe notarse de (2.43) que para aplicaciones en electromagnetismo con polarización en el eje $\mathrm{z}$, se tiene que $\varepsilon(\mathrm{x}, \mathrm{y})=1+\mathrm{q}(\mathrm{x}, \mathrm{y})$, donde $\varepsilon(\mathrm{x}, \mathrm{y})$ es la permitividad eléctrica relativa dentro del espacio de simulación y $\mathrm{q}(\mathrm{x}, \mathrm{y})$ es la función contraste. Dicha función contraste describe el material dieléctrico dentro del espacio de simulación. El espacio de simulación en $2 \mathrm{D}$ será un cuadrado unidad donde $\mathrm{R}=\{(\mathrm{x}, \mathrm{y}) \in \mathrm{IRx} \operatorname{IR} / 0 \leq \mathrm{x}$ $\left.\leq 1^{\wedge} 0 \leq \mathrm{y} \leq 1\right\}$. A cada punto $(\mathrm{x}, \mathrm{y})$ se le debe asignar un valor; 0 donde no hay dieléctrico 
$\left(\varepsilon_{\mathrm{r}}=1\right)$ y el valor de su constante dieléctrica relativa donde existe el material dieléctrico.

Por otro lado, mediante otra función se asigna la fuente electromagnética. El código permite modificar el perfil del alimentador con lo cual se pueden utilizar diferentes modelos de alimentadores. Cabe destacar que el material dieléctrico debe definirse dentro del cuadrado unidad mientras que la fuente pude colocarse afuera.

Una vez finalizada la ejecución del programa se almacenan los resultados, es decir, los valores del campo para cada punto dentro del cuadrado unidad. Por otro lado, se obtienen automáticamente las gráficas módulo y la fase del campo y el diagrama polar y cartesiano de campo lejano. Adicionalmente se genera otro gráfico con el campo dentro de la función contraste (material dieléctrico) para un mejor estudio del mismo. 


\section{CAPÍTULO 3}

\section{Antenas de apertura}

\subsection{Introducción}

Una antena lente es un sistema formado por una fuente electromagnética denominada alimentador seguido de una lente apropiadamente ubicada.

Las lentes permiten transformar las ondas esféricas provenientes del alimentador en ondas planas, modificando su amplitud y fase. De esta forma se obtiene mayor directividad y ganancia de radiación, en comparación con el alimentador aislado. En este sentido las lentes son equivalentes a los reflectores. Sin embargo, en lugar de la reflexión, el principio de operación de la lente se basa en la refracción de las ondas electromagnéticas en las superficies de la lente (en el caso de lentes homogéneas isotrópicas), o en el material dieléctrico de la lente en el caso de lentes de índice de refracción no uniformes.

La lente se coloca de tal manera que el centro de fase del alimentador esté ubicado en su punto focal, consecuentemente se obtienen rayos colimados o paralelos a la salida de la lente. El alimentador suele ser una antena de bocina que genera un frente de onda esférico o un arreglo de antenas que produce un frente de onda cilíndrico. Durante la recepción, cuando los rayos inciden en la lente, se refractan y convergen en el punto focal donde se encuentra el receptor. La Figura 3.1 muestra el funcionamiento de una antena lente en (a) transmisión y en (b) recepción. 


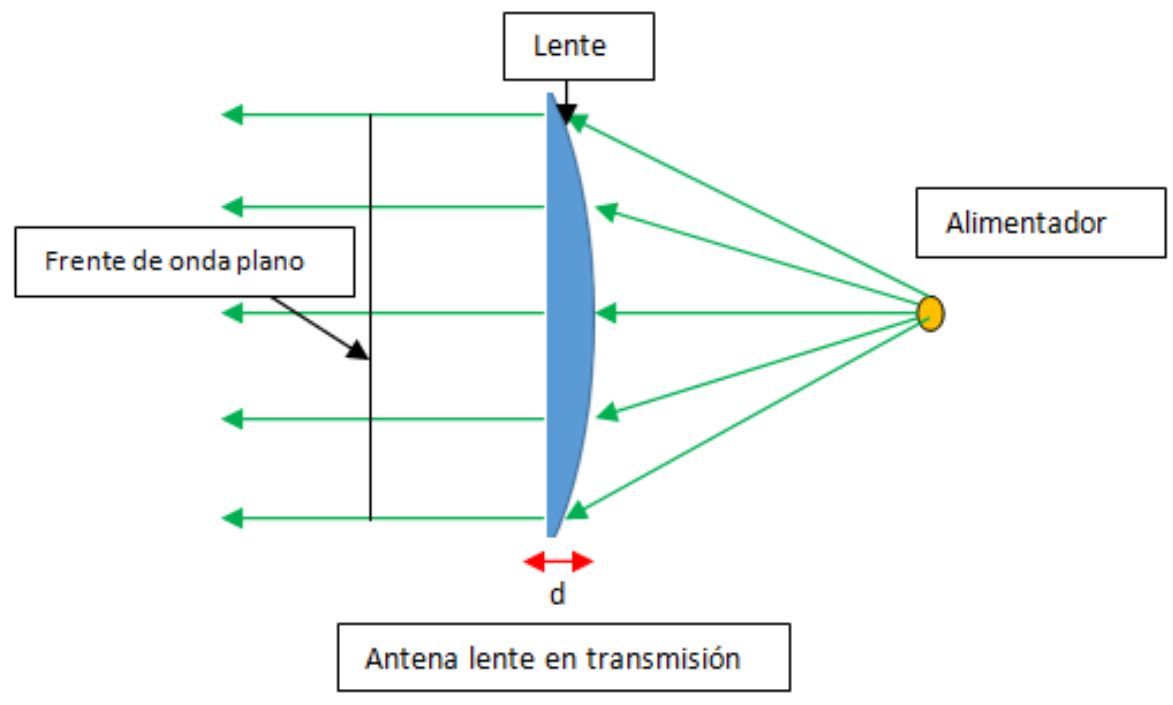

a)

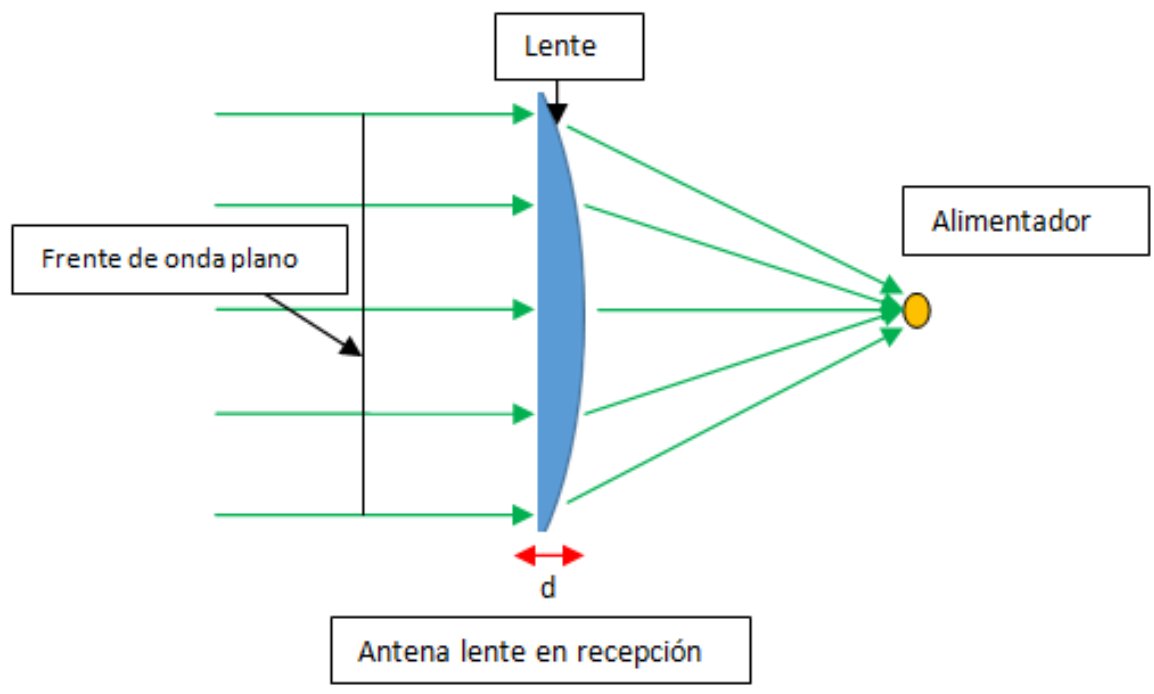

b)

Figura 3.1: Diagrama de funcionamiento de una antena lente en a) transmisión y b) recepción.

En general las antenas lente se utilizan para aplicaciones de alta frecuencia que requieren anchos de banda amplios [46]. Existen diversos tipos de antenas lente.

Las antenas lente dieléctricas son aquellas en las que las ondas de desplazamiento se retrasan con los medios de la lente. Dichas lentes pueden estar formadas por uno (lente homogénea) o varios materiales dieléctricos (lente no-homogénea).

En este capítulo se discuten las características eléctricas generales de las antenas, tales como directividad y eficiencia. Debido a que las antenas lente pueden considerarse como antenas de apertura, el análisis hace foco en dicho tipo de antenas [61-67]. 
Las antenas lente constan de dos diagramas de radiación (primario y secundario). El diagrama primario está dado por el alimentador, y el secundario está formado por el conjunto antena lente.

Para el diseño de las antenas lente es fundamental conocer sus características y observar de qué parámetros dependen.

\subsection{Antenas de apertura}

Las antenas de apertura son aquellas que utilizan superficies o aperturas para direccionar el haz electromagnético de forma tal que concentran la emisión y recepción de su sistema radiante en una dirección. Dentro de las antenas de apertura se pueden citar las antenas parabólicas y las antenas lente.

Las antenas parabólicas cuentan con un reflector de material conductor y utilizan el principio de reflexión para concentrar las ondas electromagnéticas hacia una dirección tal como se observa en la Figura 3.2 a).

Las antenas lentes, a diferencia de las antenas parabólicas, utilizan el principio de refracción para concentrar las ondas electromagnéticas. La Figura 3.2 muestra un esquema de funcionamiento de un reflector (a) y una antena lente (b).

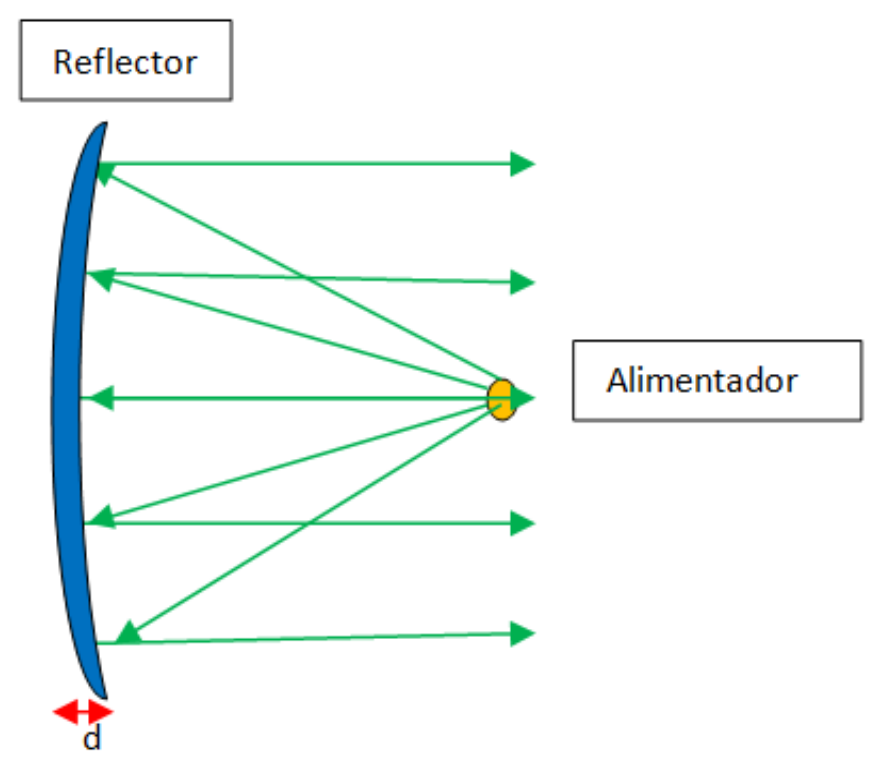

a) 


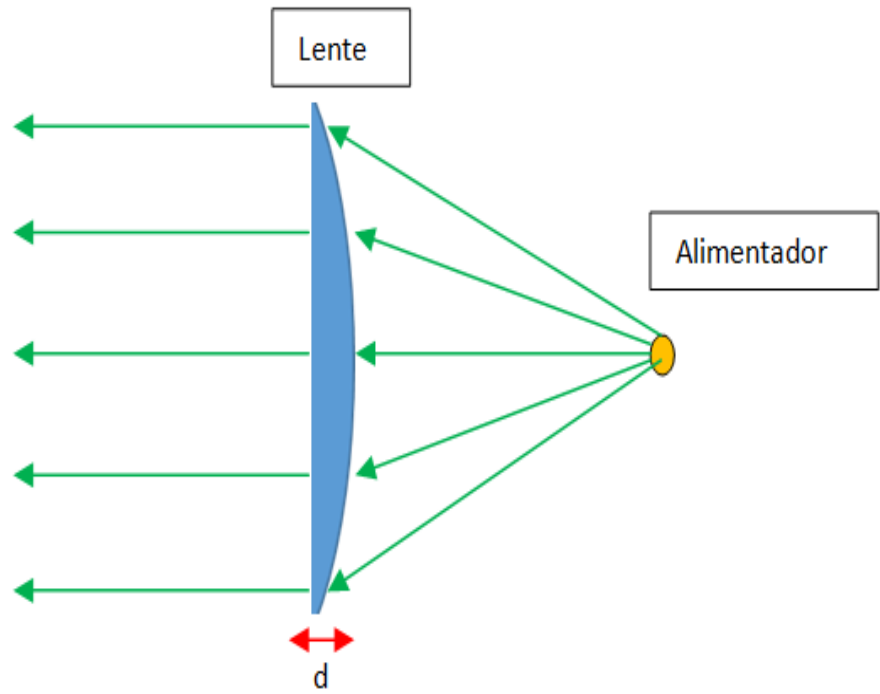

b)

Figura 3.2: Diagramas de antenas de apertura para el caso de a) Reflector y b) Antena lente.

La ganancia de dichas antenas está relacionada con la superficie de la apertura, a mayor tamaño mayor colimación del haz se tiene y por lo tanto mayor directividad.

El elemento radiante es el alimentador, el cual puede iluminar de forma directa a la apertura. En sí mismo es una antena que debe iluminar lo más eficientemente la apertura del reflector en el caso de la Fig. 3.2 a) o de la lente en la Fig. 3.2 b). El alimentador está generalmente ubicado en el foco de la parábola o la lente.

\subsubsection{Parámetros básicos de antenas}

Asociado con el campo eléctrico y magnético en la dirección de propagación está el vector de Poynting, el cual es referido como la densidad de potencia $\mathrm{S}_{\mathrm{a}}$, dada por:

$$
S_{a}=\frac{\operatorname{Re}(E x H)}{2}
$$

donde E es el campo eléctrico y H es el campo magnético.

Este permite cuantificar la densidad de potencia electromagnética en cualquier punto del espacio. Por lo tanto, la magnitud $\mathrm{S}_{\mathrm{a}}$ incidirá en la determinación de muchos de los parámetros que comúnmente definen las propiedades de la antena (directividad, ancho de haz y área efectiva) [62-64].

La distribución espacial de energía radiada por una antena es función de la posición en el espacio y está caracterizada por el llamado diagrama de radiación de la 
antena. Normalmente, para la representación gráfica del diagrama de radiación se utilizan las coordenadas esféricas [62-64].

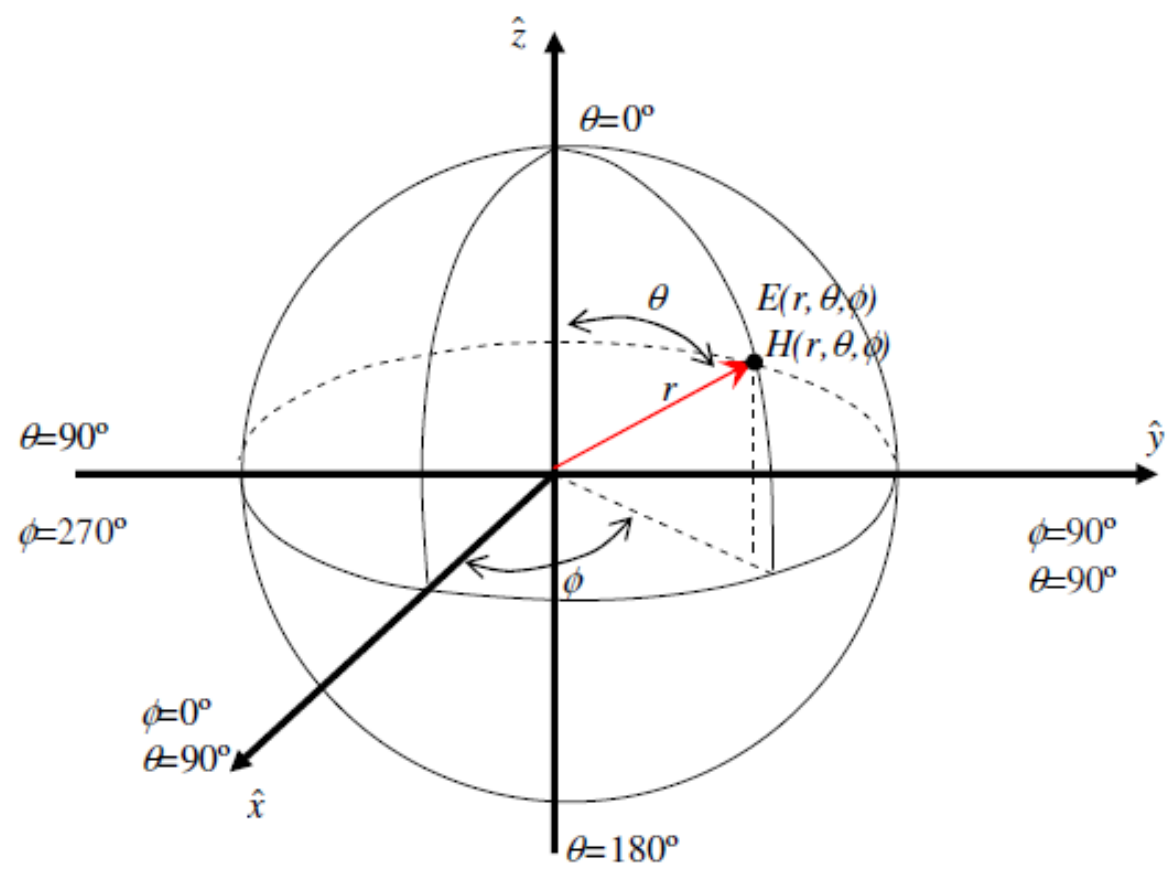

Figura 3.3: Coordenadas Esféricas.

Las variables utilizadas son la distancia al punto considerado $(r)$, ángulo de elevación $(\theta)$ y ángulo de azimut $(\varphi)$.

El diagrama de radiación de una antena es la distribución de la amplitud relativa del campo electromagnético o más comúnmente la distribución de la densidad de potencia $\left(\mathrm{S}_{\mathrm{a}}\right)$, también aplicado en forma recíproca a antenas utilizadas como receptoras.

Si se considera un transmisor colocado en el origen del sistema mostrado en la Figura 3.3 y se elige $r$ lo suficientemente grande para considerar que el frente de onda en el diferencial de área (dA) es plano, es posible considerar que se está observando una zona en el campo lejano. Esta región es de un particular interés, no sólo porque matemáticamente puede simplificar los procesos a estudiar, sino porque generalmente las interacciones con otros sistemas ocurren a grandes distancias, es decir, en el campo lejano. 


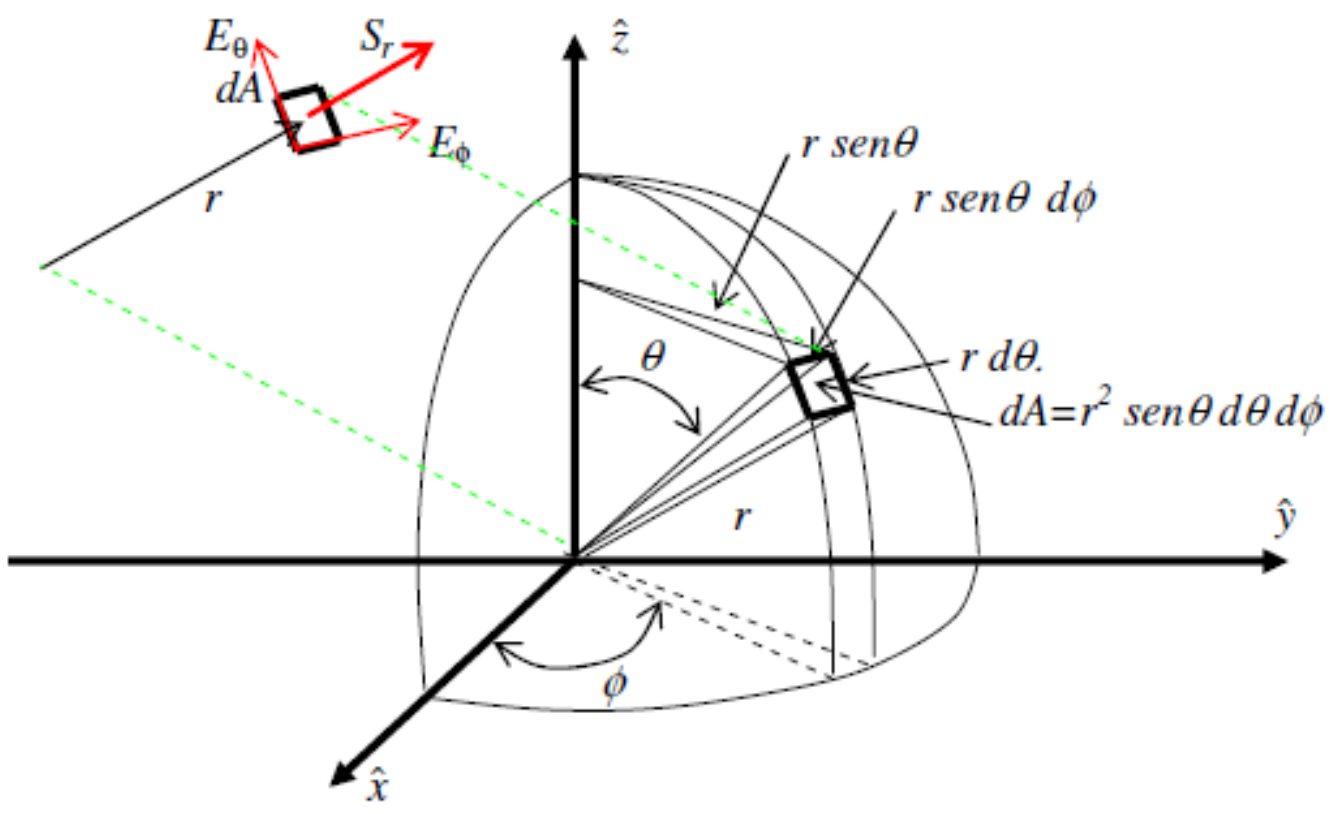

Figura 3.4: Radiación de Radiofuente en coordenadas Esféricas.

En una onda plana, los vectores de campo eléctrico E y campo magnético $\mathrm{H}$ en el campo lejano son perpendiculares entre sí y se producen en un plano normal al vector $r$.

$$
\vec{E}=\hat{\theta} \cdot E_{\theta}+\hat{\varphi} \cdot E_{\varphi}
$$

Siendo $Z_{\mathrm{i}}$ la impedancia intrínseca del medio, se tiene:

$$
\vec{H}=\frac{1}{Z_{i}} \vec{E}=\frac{1}{Z_{i}}\left(\hat{\theta} \cdot E_{\theta}+\hat{\varphi} \cdot E_{\varphi}\right)
$$

Luego la densidad de potencia $\mathrm{S}_{\mathrm{r}}$ en el área considerada tiene solo componente radial, y no en las direcciones $\theta$ y $\varphi$ con lo que:

$$
S_{r}=\frac{\operatorname{Re}(E x H)}{2}=\frac{1}{2 Z_{i}}\left(\left|E_{\theta}\right|^{2}+\left|E_{\varphi}\right|^{2}\right)
$$

A grandes distancias de la antena, el campo radiado $E_{\theta}\left(\mathrm{y}\right.$ su similar $\left.E_{\varphi}\right)$ puede ser descrito por una función de propagación esférica $\frac{e^{-j \beta r}}{r}$, características de una fuente puntual, multiplicada por la función dirección $f(\theta, \varphi)$ :

$$
E_{\theta}=\frac{e^{-j \beta r}}{r} f_{1}(\theta, \varphi)
$$




$$
E_{\varphi}=\frac{e^{-j \beta r}}{r} f_{2}(\theta, \varphi)
$$

donde $\beta=\frac{2 \pi}{\lambda}$, siendo $\lambda$ la longitud de onda de la radiación.

El flujo de potencia en el campo lejano está dado por:

$$
S_{r}=\frac{1}{2 Z_{i} r^{2}}\left(\left|f_{1}(\theta, \varphi)\right|^{2}+\left|f_{2}(\theta, \varphi)\right|^{2}\right)
$$

La densidad de potencia $\mathrm{S}_{\mathrm{r}}(r, \theta, \varphi)$ describe la propiedades de direccionalidad de la antena. Generalmente es conveniente usar una función independiente de la distancia $r$ conocida como intensidad de radiación o diagrama de radiación $F(\theta, \varphi)$ dada por $[62,63]$ :

$$
F(\theta, \varphi)=r^{2} \cdot S_{r}=\frac{1}{2 Z_{i}}\left(\left|f_{1}(\theta, \varphi)\right|^{2}+\left|f_{2}(\theta, \varphi)\right|^{2}\right)
$$

Donde $F(\theta, \varphi)$ es ahora expresado en watt por unidad de ángulo sólido (watt por estereorradián). Es costumbre normalizar el máximo valor de $F(\theta, \varphi)$ a la unidad, en ese caso el diagrama es referido como diagrama de radiación normalizado $F_{n}(\theta, \varphi)$.

$$
F_{n}(\theta, \varphi)=\frac{F(\theta, \varphi)}{F_{\max }(\theta, \varphi)}=\frac{S_{r}}{S_{r \max }}
$$

En la Figura 3.5 a), b) y c) se puede ver las distintas formas de presentar un diagrama $F_{n}(\theta, \varphi)$ donde la escala de amplitud utilizada está en dB y la otra coordenada es una medida de ángulos. Este formato permite convenientemente la interpretación de la radiación de los lóbulos. 

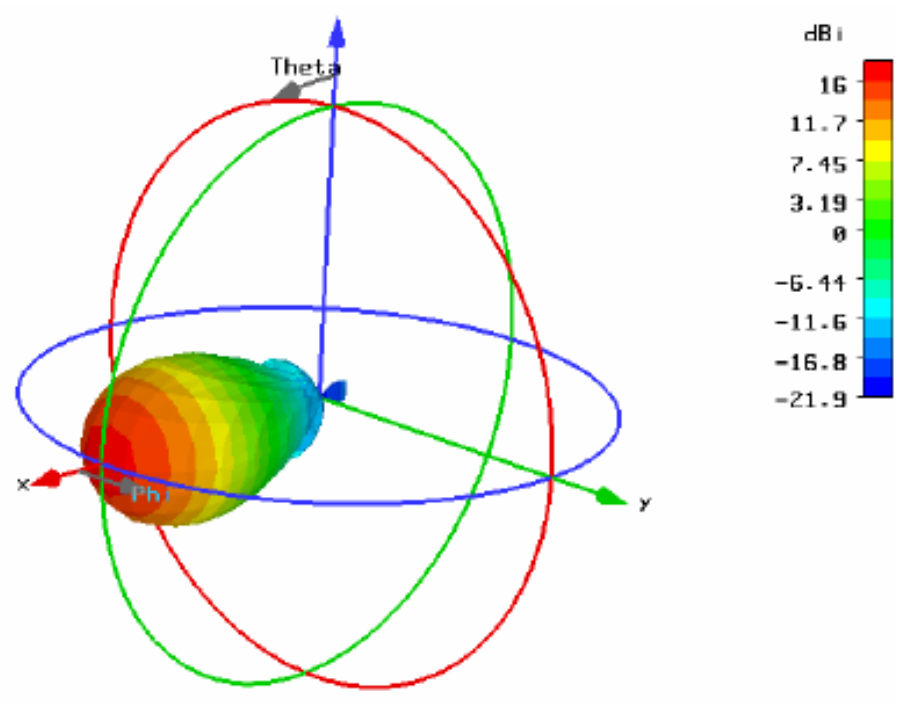

a)

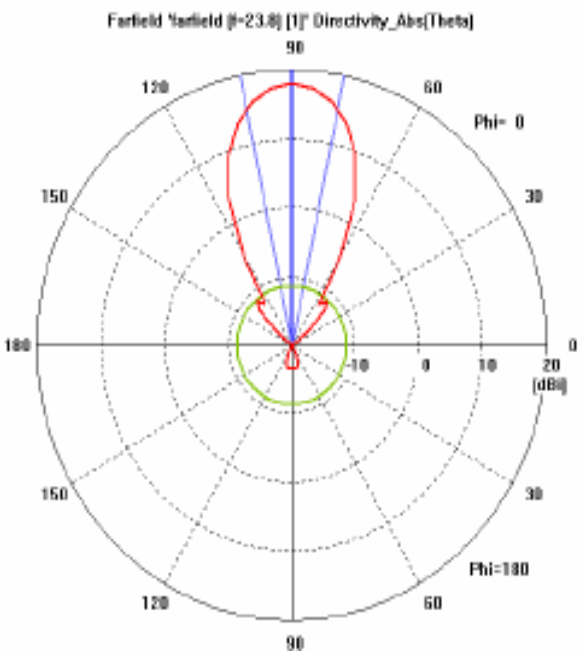

a)

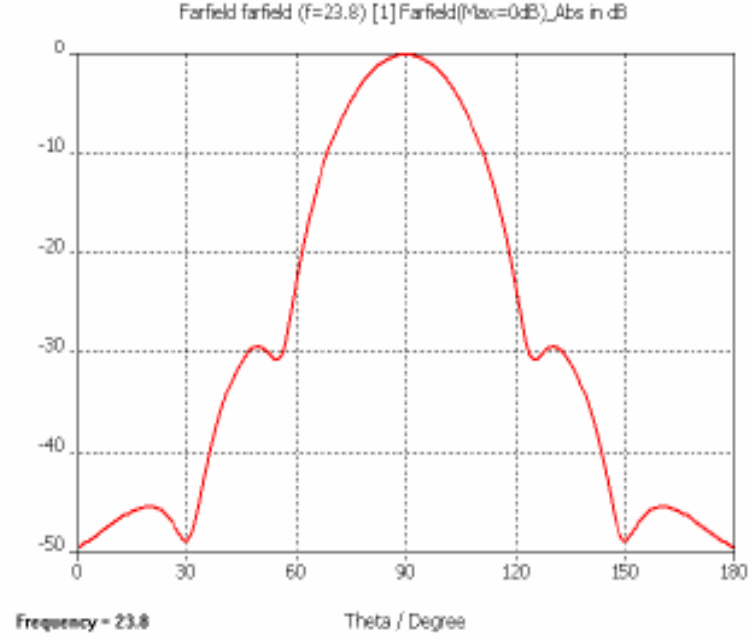

c)

Figura 3.5: a) Diagrama en 3D, b) Diagrama Polar, c) Diagrama Cartesiano.

En la Figura 3.5 se observa un diagrama de radiación direccional, obtenido con el software de simulación comercial Feko [51]. En los diagramas se pueden distinguir el lóbulo principal, los lóbulos laterales y lóbulos traseros (lóbulos menores). El primer lóbulo lateral, es el primer lóbulo que aparece a ambos lados del haz principal.

De estos diagramas se pueden extraer varios valores numéricos que especifican parámetros que caracterizan a la antena, como veremos a continuación. 


\subsubsection{Directividad}

Como se mencionó previamente, la finalidad de una lente es concentrar la potencia radiada por el alimentador en una determinada dirección del espacio con un diagrama que cumpla determinadas especificaciones, como pueden ser directividad, nivel de lóbulos secundarios y polarización cruzada.

La directividad $D(\theta, \varphi)$ de una antena [61-67], en una determinada dirección, es el cociente entre el diagrama de radiación $F_{n}(\theta, \varphi)$ en esa dirección y su valor promedio.

$$
D(\theta, \varphi)=\frac{F_{n}(\theta, \varphi)}{\frac{1}{4 \pi} \iint_{4 \pi} F_{n}(\theta, \varphi) \cdot d \Omega}
$$

Usando la ecuación 3.10 la directividad puede ponerse en función de las densidades de potencia, quedando:

$$
D(\theta, \varphi)=\frac{4 \pi S_{r}(\theta, \varphi)}{\iint_{4 \pi} S_{r}(\theta, \varphi) \cdot d \Omega}
$$

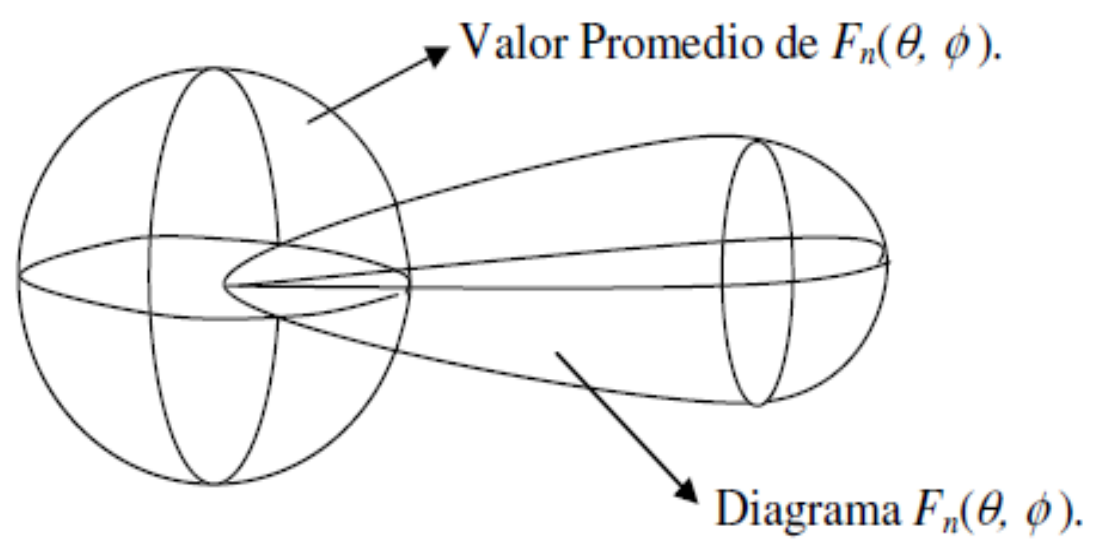

Figura 3.6: Diagramas de radiación en 3D.

En particular tiene importancia en el diseño de la antena el cálculo de directividad máxima $\mathrm{D}_{\mathrm{o}}$ que ocurre cuando $F_{n}(\theta, \varphi)=1$. Luego, de la ecuación 3.11 se tiene:

$$
D_{0}=\frac{4 \pi}{\iint_{4 \pi} F_{n}(\theta, \varphi) \cdot d \Omega}=\frac{4 \pi}{\Omega_{p}}
$$

La directividad en una dirección $(\theta, \varphi)$ puede escribirse como: 


$$
D(\theta, \varphi)=D_{0} F_{n}(\theta, \varphi)
$$

En una antena con un lóbulo principal en la dirección z como se muestra en la figura 3.7, el diagrama del ángulo sólido $\Omega_{\mathrm{p}}$ es igual aproximadamente al producto de los anchos de haz de potencia mitad en dos ejes ortogonales [62]:

$$
\Omega_{p} \approx \alpha_{x z} \cdot \alpha_{y z}
$$

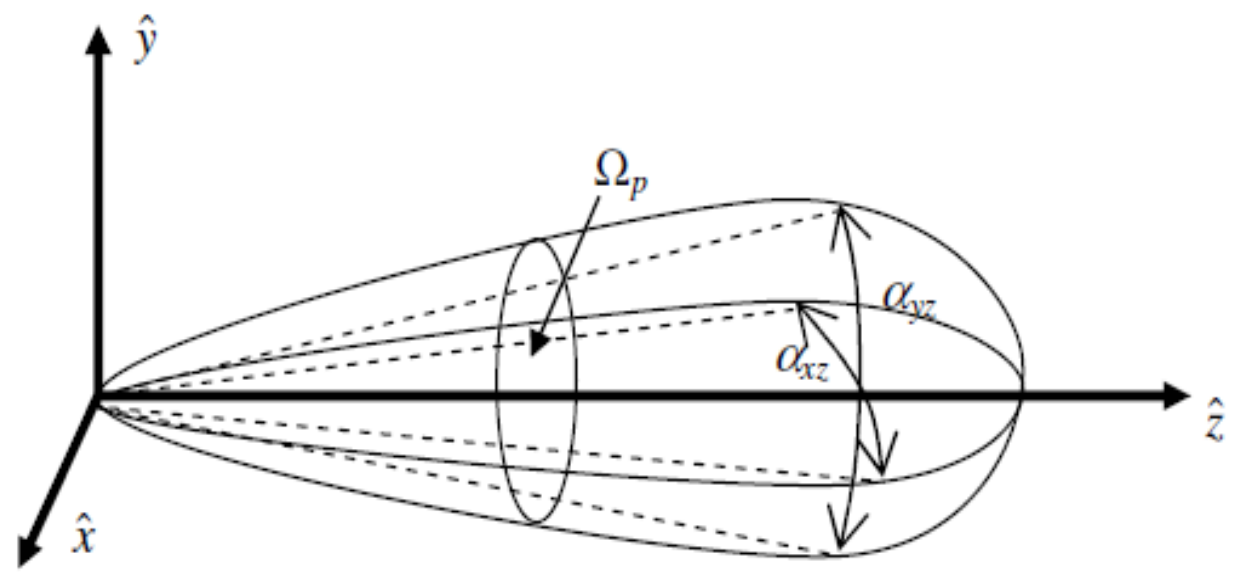

Figura 3.7: Lóbulo principal de radiación.

donde $\alpha_{\mathrm{yz}}$ es el ancho del lóbulo de $3 \mathrm{~dB}$ en el plano yz y $\alpha_{\mathrm{xz}}$ es el ancho del lóbulo de $3 \mathrm{~dB}$ en el plano $\mathrm{xz}$.

Como consecuencia:

$$
D(\theta, \varphi)=\frac{4 \pi}{\Omega_{p}} \approx \frac{4 \pi}{\alpha_{x z} \cdot \alpha_{y z}}
$$

Esta aproximación permite estimar la directividad a través de los ángulos de potencia mitad de los diagramas de radiación de planos ortogonales.

\subsubsection{Eficiencia de apertura de antena}

Si $P_{t}$ es la potencia total que irradia la antena, una parte $P_{o}$ ilumina la apertura (en este caso la lente), y parte de $P_{t}$ se dispersa, luego se define la eficiencia de apertura $o$ radiación $[62,63]$ como: 


$$
\eta_{a}=\frac{P_{0}}{P_{t}}
$$

La eficiencia de apertura de un sistema de antena reflectora es dependiente de varios factores y puede expresarse como:

$$
\eta_{a}=\eta_{i} \cdot \eta_{s} \cdot \eta_{c}
$$

Donde $\eta_{\mathrm{i}}$ es la eficiencia de iluminación, $\eta_{\mathrm{s}}$ es la eficiencia de "spillover" o sobreiluminación y $\eta_{\mathrm{c}}$ es la eficiencia de construcción o alineación, y donde los valores que toma la eficiencia de apertura son $0 \leq \eta_{\mathrm{a}} \leq 1$.

La eficiencia de iluminación cuantifica con qué uniformidad el alimentador principal ilumina la superficie de la lente, y se puede definir como [62, 63]:

$$
\eta_{i}=\frac{2 \cdot \operatorname{cotg}^{2}\left(\frac{\theta_{0}}{2}\right) \cdot\left|\int_{0}^{\theta_{0}}\left(\left|F_{\theta}\right|+\left|F_{\varphi}\right|\right) \cdot \tan \left(\frac{\theta}{2}\right) \cdot d \theta\right|^{2}}{\int_{0}^{\theta_{0}}\left(\left|F_{\theta}\right|+\left|F_{\varphi}\right|\right) \cdot \sin (\theta) \cdot d \theta}
$$

Mientras que la eficiencia de "spillover" mide cuánto ilumina el alimentador fuera del reflector, en su objetivo de iluminar eficientemente la superficie del mismo. Su expresión es la siguiente:

$$
\eta_{s}=\frac{\int_{0}^{\theta_{0}}\left(\left|F_{\theta}\right|+\left|F_{\varphi}\right|\right) \cdot \sin (\theta) \cdot d \theta}{\int_{0}^{\pi}\left(\left|F_{\theta}\right|+\left|F_{\varphi}\right|\right) \cdot \sin (\theta) \cdot d \theta}
$$

siendo $F_{\theta} y F_{\varphi}$ los diagramas de amplitud en los planos $\theta$ y $\varphi$ respectivamente y $\theta_{0}$ es el ángulo formado entre los bordes de la lente y su foco. 


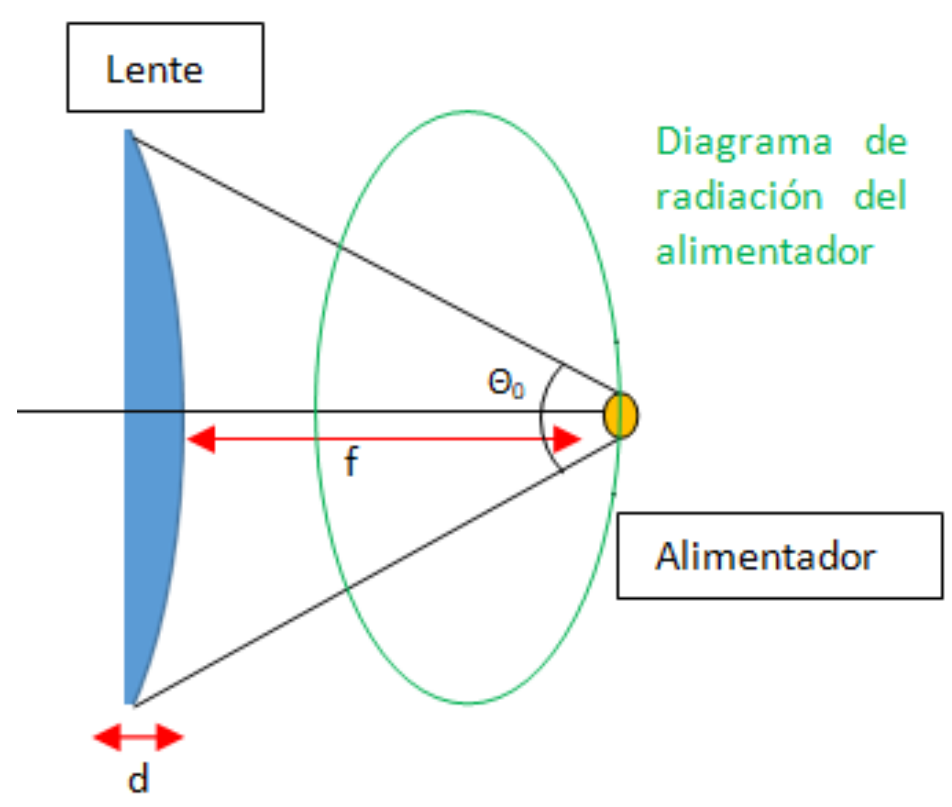

a)

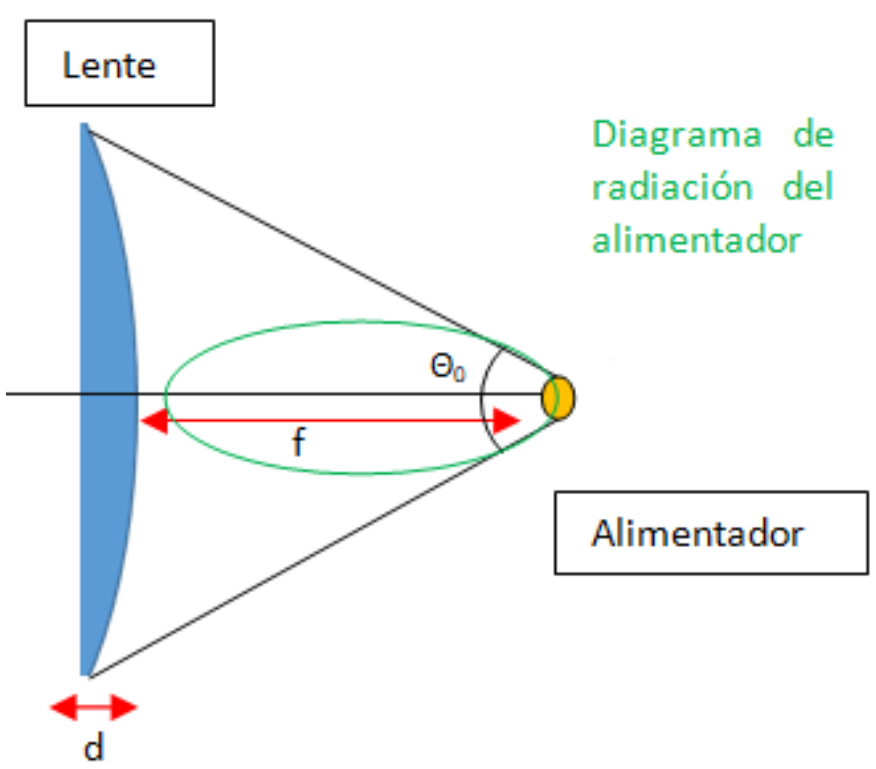

b)

Figura 3.8: a) Baja eficiencia de Spillover b) Baja eficiencia de iluminación.

Se observa de las Figuras 3.8 a) y b), que hay un factor de compromiso entre ambas eficiencias. Cuando se ilumina en mayor proporción la superficie de la lente la eficiencia de iluminación aumenta, mientras la de "spillover" disminuye y viceversa.

Además, se puede ver que ambas eficiencias son íntimamente dependientes del diseño del alimentador principal. Existe un punto donde la eficiencia total dada por (3.17) es máxima. 
A partir de la directividad (3.15) y la eficiencia (3.16) es posible definir la ganancia $G(\theta, \varphi)$ de la antena, dada por

$$
G(\theta, \varphi)=\eta_{a} \cdot D(\theta, \varphi)
$$

Teniendo en cuenta la eficiencia, se puede escribir la ganancia máxima como $[62,63]$ :

$$
G_{M a x}=\frac{4 \pi}{\lambda^{2}} A_{g e o m} \cdot \eta_{a}
$$

Donde $\lambda$ es la longitud de onda, $A_{\text {geom }}$ es el área geométrica de la lente y $\eta_{a}$ es la eficiencia total.

Para una lente circular de diámetro $D$, se tiene una ganancia máxima de:

$$
G_{\text {Max }}=\left(\frac{\pi D}{\lambda}\right)^{2} \cdot \eta_{a}
$$

\subsubsection{Decaimiento en bordes}

Se observa que la distribución de campos en la apertura de la lente tiene una fase constante, debido a la igualdad de caminos, mientras que la amplitud no es uniforme. Esta falta de uniformidad es debida a dos causas [61]:

Por un lado, la dependencia con $1 / r^{\prime}$, debida a que el campo decae como el de una onda esférica hasta incidir en la lente. Un punto de igual fase de la onda esférica generada por la fuente recorre una mayor distancia cuando llega al borde de la lente.

Si se supone una lente plana o bien una lente cuya la distancia focal es mayor al espesor de la lente, la relación de densidades de potencia para un ángulo $\theta$ ' respecto al eje de la lente $\left(\theta^{\prime}=0\right)$ está dada por

$$
\tau_{1}=\frac{S_{r}\left(\theta^{\prime}\right)}{S_{r}\left(\theta^{\prime}=0\right)}=\frac{f^{2}}{r^{\prime 2}}
$$

Donde $\mathrm{S}_{\mathrm{r}}\left(\theta^{\prime}\right)$ son las densidades de potencia para un ángulo $\theta^{\prime}$ respecto al eje del paraboloide $\left(\theta^{\prime}=0\right)$.

Otra causa del decaimiento es debido a la direccionalidad del diagrama de radiación del alimentador $\mathrm{D}_{\mathrm{f}}\left(\theta^{\prime}\right)$. Si suponemos que éste irradia el máximo en la dirección del eje, tendremos 


$$
\tau_{12}=\frac{D_{f}\left(\theta^{\prime}\right)}{D_{f}\left(\theta^{\prime}=0\right)}
$$

El decaimiento en bordes es un parámetro importante ya que permitirá determinar la eficiencia máxima entre eficiencia por spillover y eficiencia por iluminación.

\subsubsection{Relación f $/ \mathbf{D}_{\mathbf{a}}$}

La relación entre la distancia focal $f$ y el diámetro de la lente es de fundamental importancia para el diseño de la antena lente.

Analizando la Figura 3.9 es posible observar que, al modificar la distancia focal $f$, el ángulo que se forma entre el alimentador y el borde de la lente $\beta$ también se modifica. Esto hace que, dado un diagrama de radiación propio del alimentador, se modifique la iluminación de la lente en función de dicha relación, variando así las eficiencias vistas en el punto 3.3.3.

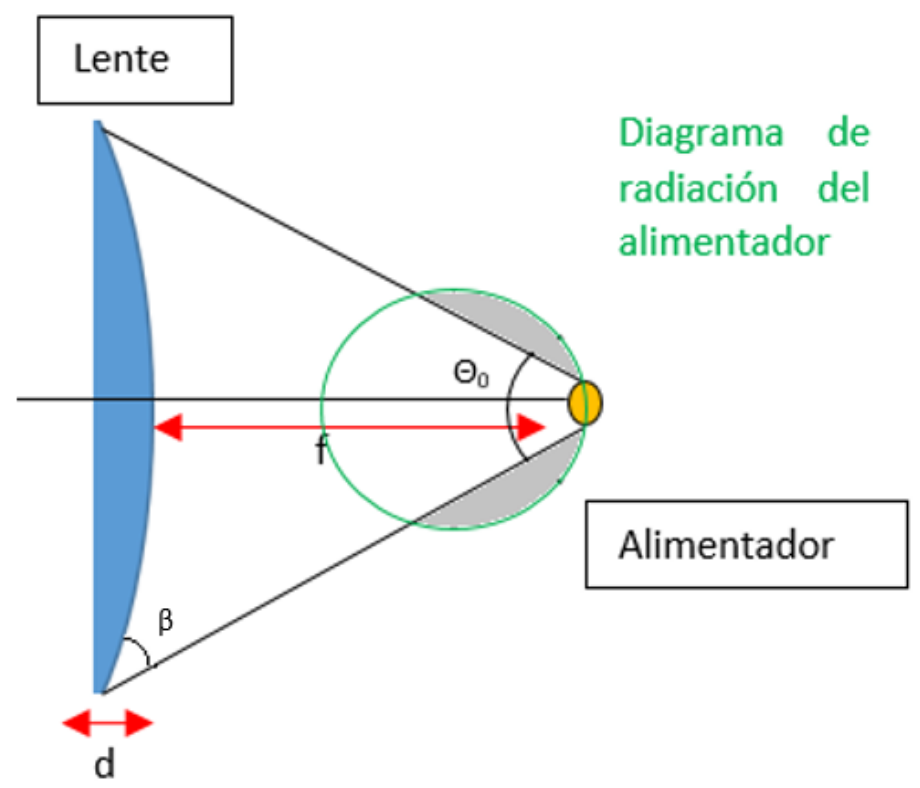

a) 


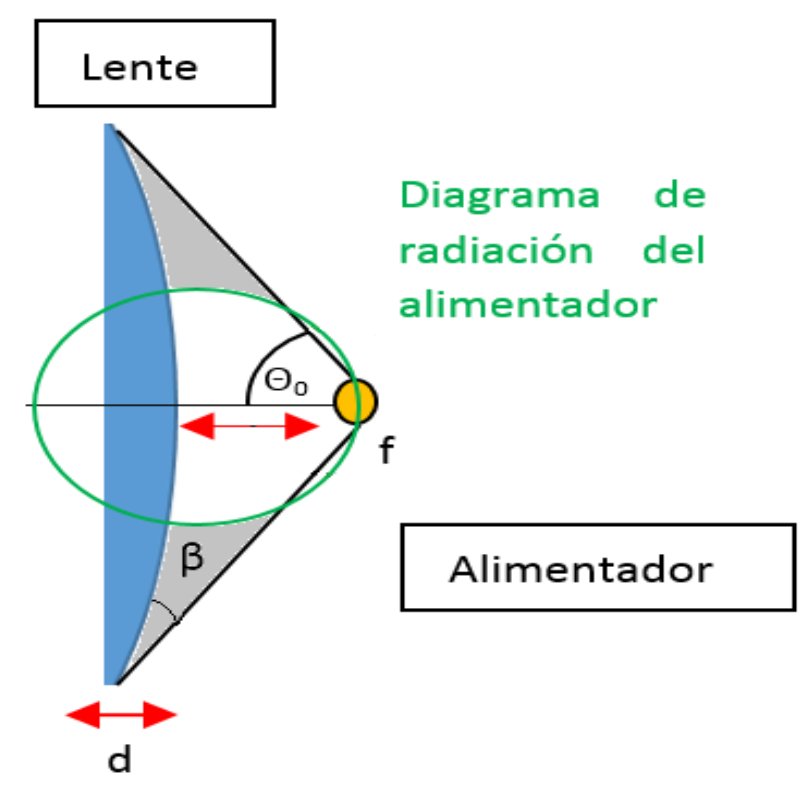

b)

Figura 3.9: a) Relación $f / D_{a}$ Alto b) Relación $f / D_{a}$ baja.

Para valores de $\mathrm{f} / \mathrm{D}_{\mathrm{a}}$ bajos $\left(\mathrm{f} / \mathrm{D}_{\mathrm{a}} \leq 0,25\right)$ el alimentador está muy próximo a la lente, con lo cual las pérdidas por desbordamiento (Spillover) son muy bajas; por el contrario, la iluminación de la apertura presenta un fuerte decaimiento en los bordes, por lo cual no se utiliza eficientemente la superficie de la lente. Valores de $f / D_{a}$ elevados $\left(f / D_{a} \geq 0,5\right)$ permiten una buena iluminación de la apertura, pero tienen elevadas pérdidas por desbordamiento [61].

En la figura 3.10 se representa una gráfica de la variación de las diferentes eficiencias en función del parámetro $\mathrm{f} / \mathrm{D}_{\mathrm{a}}$ para un alimentador tipo bocina [62]. Se observa que hay un punto en el que se optimiza la eficiencia total de la antena para ese alimentador en concreto. Al cambiar el alimentador variará la forma de las curvas y, por tanto, el punto óptimo. Cuando se requiera la máxima directividad de un conjunto antena lente debe determinarse dicho punto. Sin embargo, para aplicaciones donde se necesitan valores excepcionalmente bajos por ejemplo nivel de lóbulos secundarios, se utilizarán otros puntos de trabajo que no son óptimos desde el punto de vista de la eficiencia.

Se encuentra en general que, para iluminaciones en los bordes del orden de -10 $\mathrm{dB}$ respecto al centro, se suelen obtener los mejores diseños en cuanto a ganancia, mientras que en torno a $-20 \mathrm{~dB}$ es el nivel habitual para una buena relación lóbulo principal a secundario o NLPS (Nivel Lóbulo Principal a Secundario) [61]. 


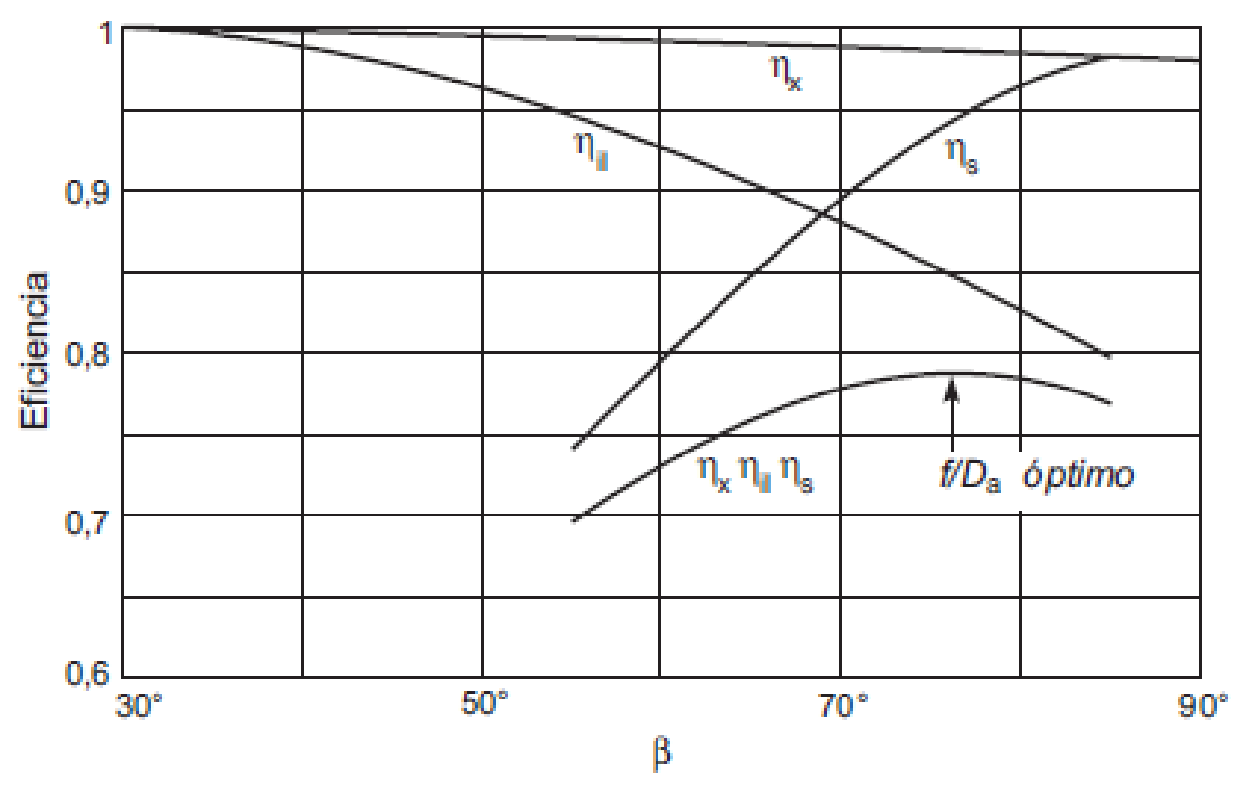

Figura 3.10: Variación de los parámetros de eficiencia en función del ángulo $\beta$.

\subsubsection{Perdidas por reflexión y transmisión}

Además de las eficiencias vistas en el apartado interior, otro factor propio de las lentes es la atenuación producida por las pérdidas en el medio y por la energía reflejada en la superficie de la lente.

Para incidencia normal sobre un dieléctrico el coeficiente de reflexión $\rho$, vale

$$
\rho=\frac{n-1}{n+1}
$$

Donde $n=\sqrt{\varepsilon_{r}}$ y $\varepsilon_{r}$ es la permitividad dieléctrica relativa del material.

Las pérdidas totales por reflexión se obtendrán integrando sobre todas las direcciones de incidencia los coeficientes de reflexión para ambas polarizaciones. Para la mayoría de las lentes puede utilizarse el siguiente valor aproximado [61]

$$
L_{\text {reflexión }}=8,69\left(\frac{n-1}{n+1}\right)^{2}
$$

Las pérdidas de propagación del material dependen del ángulo $\delta$ de pérdida dieléctrica. Dicho ángulo depende exclusivamente del material. Se define el factor de disipación como $\mathrm{FD}=\operatorname{Tan} \delta$. 
Las pérdidas por propagación en el material de la lente dependen del camino óptico de cada rayo y deben integrarse sobre toda la lente. Tomando el espesor mayor de la lente $d$, las pérdidas están dadas por [61]

$$
L_{\text {transmisión }}=8,69 \frac{1}{2} k_{0} \sqrt{\varepsilon_{r}} \tan \delta d
$$

Para lentes dieléctricas se tiene que mientras mayor es la permitividad eléctrica relativa del material, mayores son las pérdidas por reflexión. Sin embargo, dado que a su vez la lente resulta más delgada, las pérdidas dentro del material disminuyen. 


\section{CAPÍTULO 4}

\section{Lentes clásicas dieléctricas}

\subsection{Introducción}

En términos generales, una lente es un dispositivo de refracción (es decir, una discontinuidad en el medio) que reconfigura una distribución de energía transmitida. Eso es válido tanto para ondas Ultra violeta (UV), ondas de luz, infrarrojas (IR), microondas, ondas de radio, o incluso ondas sonoras.

En una antena lente las ondas esféricas provenientes de la fuente son transformadas en ondas planas debido a los retardos de fase que genera la lente, aumentando la directividad de todo el sistema. En una antena lente transmisora es deseable convertir ondas esféricas divergentes en un haz de ondas planas; y en una antena lente receptora es necesario recolectar rayos paralelos entrantes y concentrarlos en un $p$ unto, enfocando así la energía. Para lograr dicho efecto se diseñan lentes con diferentes perfiles.

En el presente Capítulo se indica una posible clasificación de lentes para aplicaciones en microondas. Posteriormente se aborda el estudio las de lentes clásicas dieléctricas (cuyos diseños provienen de la óptica geométrica) y sus principales características. Las lentes planas se estudian en el Capítulo 6.

\subsection{Clasificación de Lentes}

Existen diversas clasificaciones para las lentes. La Figura 4.1 muestra un diagrama de las diferentes clasificaciones de las lentes [46]. 


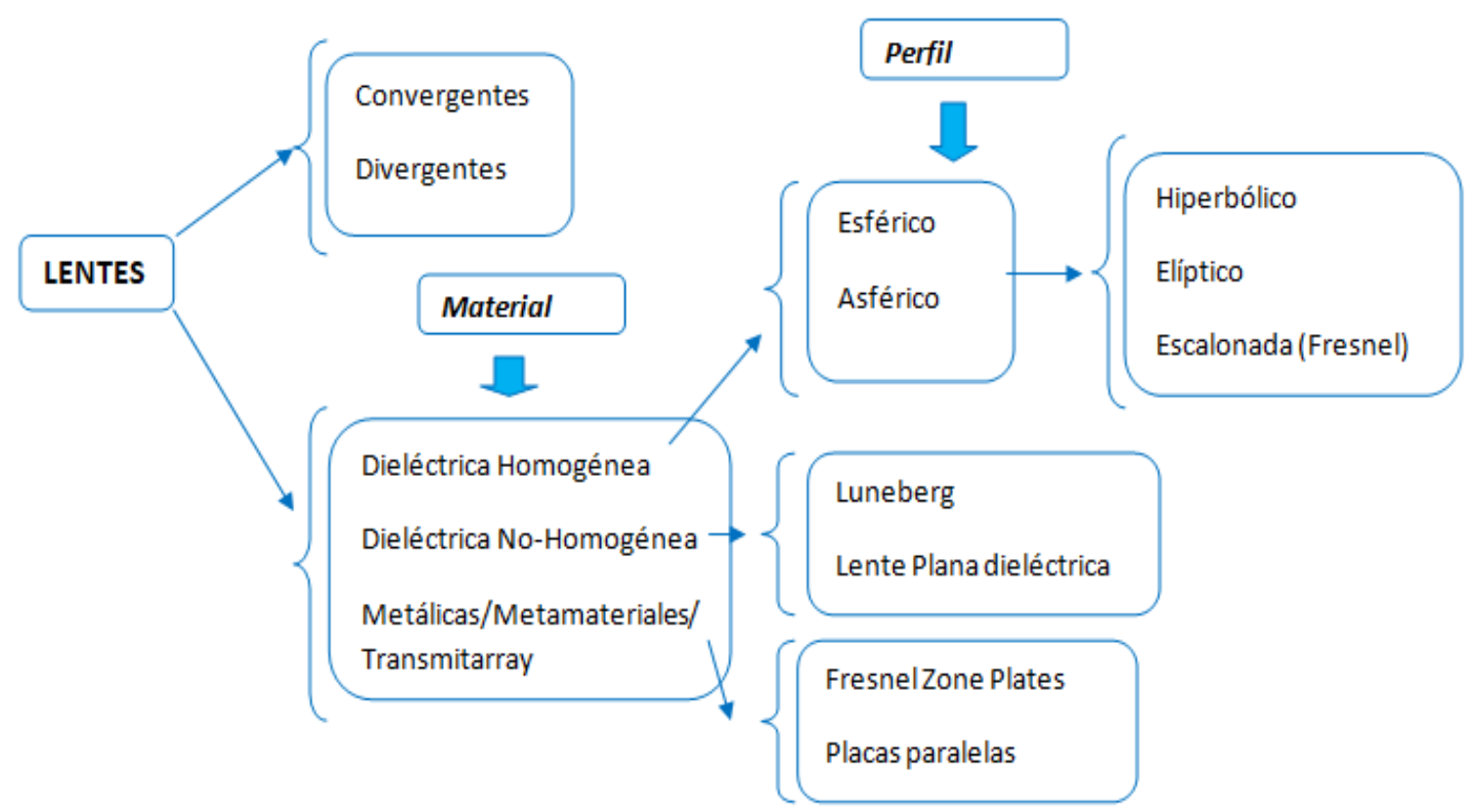

Figura 4.1: Clasificación de Lentes.

Debido a que el objeto de estudio son las antenas lente, solo se estudian las lentes convergentes ya que, como se explicó en el Capítulo 3, el objetivo de la lente es concentrar la energía en el alimentador tanto para emisión como para recepción.

Una primera gran división podría hacerse según el tipo de material. Las lentes homogéneas son aquellas que están formadas por un mismo material dieléctrico (igual permitividad relativa en todo el volumen del material) y las lentes no homogéneas pueden tener diferentes permitividades.

Existen otro tipo de lentes que incluyen materiales conductores $[9,10]$.

Según su perfil, las lentes dieléctricas homogéneas se dividen en varios grupos. Según la bibliografía clásica [2] podemos considerar las lentes esféricas y las lentes asféricas.

\subsubsection{Lentes Homogéneas}

\subsubsection{Lentes Esféricas}

Uno de los diseños más simples es la de la lente de perfil esférico. Se puede obtener su ecuación a partir de la óptica geométrica intersectando dos circunferencias de radio $R_{1}$ y $R_{2}$ [2]. 
Se pueden aplicar dos modelos para el estudio de las lentes esféricas. El modelo de lente delgada y el modelo de lente gruesa.

\section{a) Modelo lente delgada}

El modelo de la lente delgada supone que el espesor $d$ de la lente es pequeño frente a los radios de curvatura de las superficies reflectoras y las distancias s y s'.

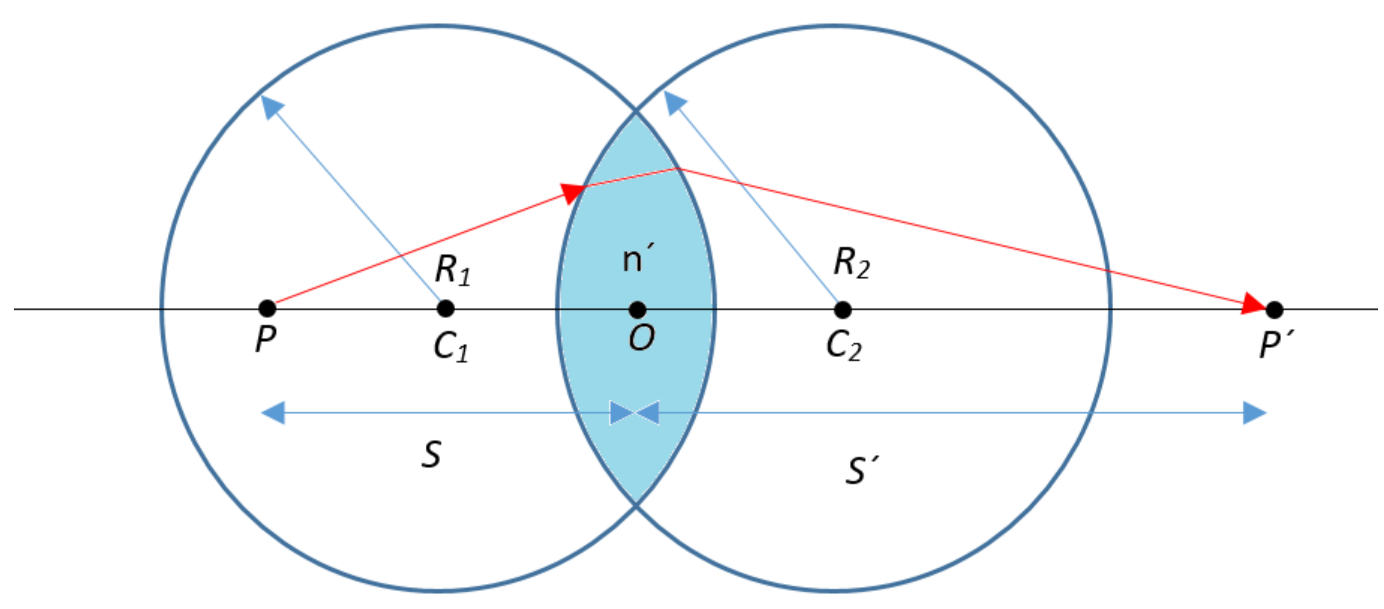

Figura 4.2: Diseño de lente esférica biconvexa delgada.

Si se considera una lente dieléctrica que está inmersa en el aire $(n=1)$ se puede utilizar la ecuación 4.1 para el diseño de la lente [2].

$$
\frac{1}{s}-\frac{1}{s^{\prime}}=\left(n^{\prime}-1\right)\left(\frac{1}{R_{1}}-\frac{1}{R_{2}}\right)
$$

$\mathrm{n}^{\prime}$ : Índice de refracción de la lente.

s, s': Son las distancias del objeto y la imagen respectivamente al origen $\mathrm{O}$, situado en el centro de la lente. Su unidad de medida en el Sistema Internacional (S.I.) es el metro (m).

$\mathrm{R}_{1}$ y $\mathrm{R}_{2}$ : Son los radios de curvatura de la primera y de la segunda superficie refractara de la lente respectivamente. Su unidad de medida en el S.I. es el metro (m)

Cabe destacar que para obtener la ecuación 4.1 se utiliza Óptica Geométrica y se aplica la aproximación paraxial. La aproximación paraxial se utiliza para el cálculo de sistemas ópticos, suponiendo que las trayectorias de los rayos de luz forman ángulos pequeños con el eje óptico.

En la aproximación paraxial de primer orden, el seno y la tangente de un ángulo se aproximan por el ángulo mismo (en radianes), y el coseno por uno. 


$$
\begin{aligned}
& \sin (\theta) \sim \theta \\
& \tan (\theta) \sim \theta \\
& \cos (\theta) \sim 1
\end{aligned}
$$

El foco objeto $F$ es el punto en el que hay que colocar el objeto para que los rayos salgan paralelos de la lente. A la distancia entre el origen y el foco objeto se la denomina distancia focal objeto $f$. Matemáticamente, $s^{\prime}=\infty \Rightarrow f=s$

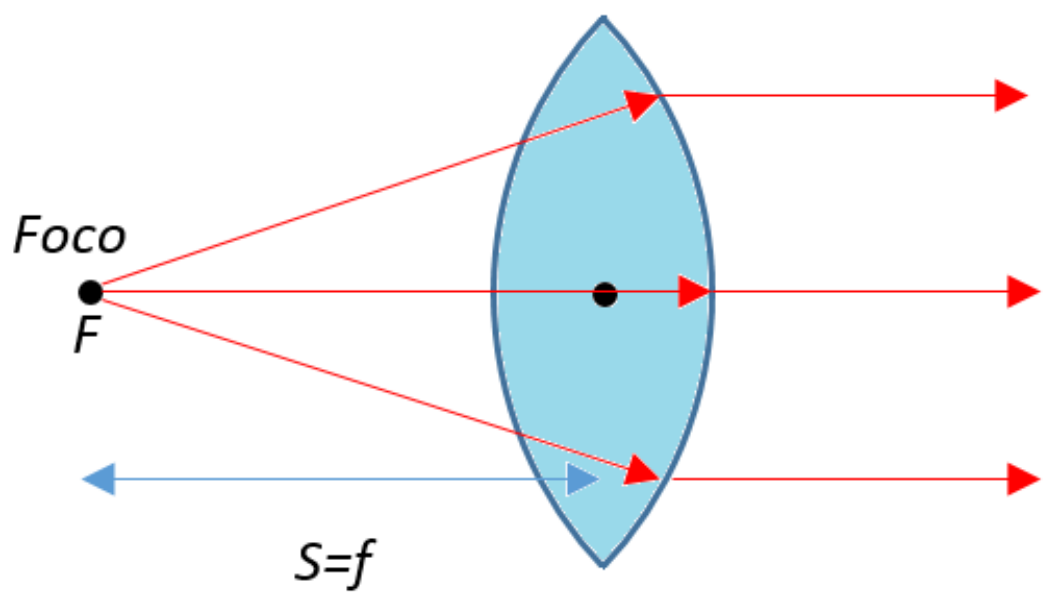

Figura 4.3: Lente biconvexa.

En este caso la ecuación $\mathrm{X}$ se reduce a

$$
\frac{1}{f}=\left(n^{\prime}-1\right)\left(\frac{1}{R_{1}}-\frac{1}{R_{2}}\right)
$$

La ecuación 4.2 es conocida como la ecuación del constructor de lentes.

Se puede diseñar la lente plano convexa haciendo que uno de los radios de curvatura tienda a infinito. 


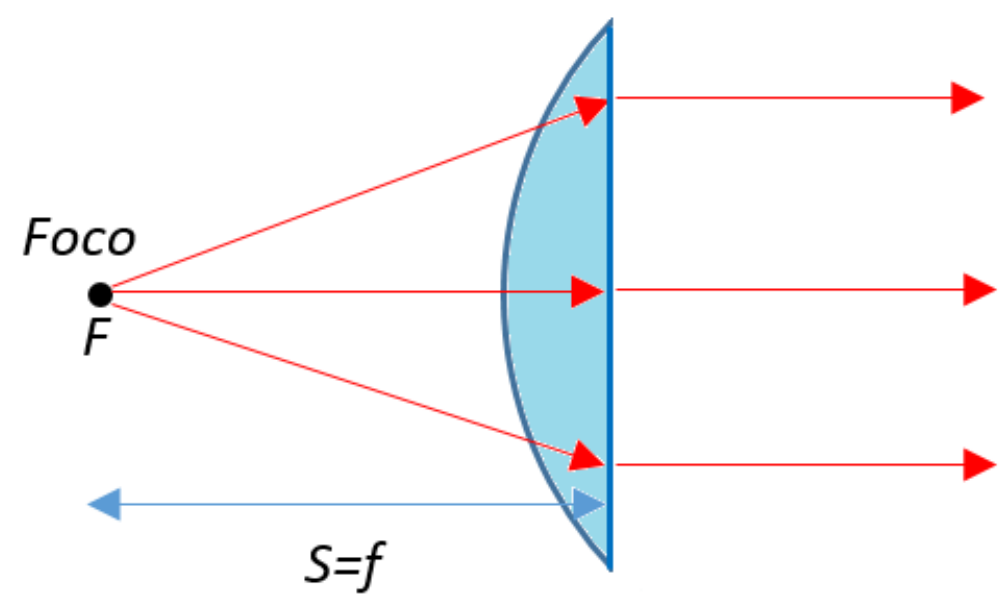

Figura 4.4: Lente plano convexa.

Utilizando la aproximación de lente delgada la ecuación que relaciona los parámetros de la lente plano-convexa está dada por:

$$
\frac{1}{f}=\left(n^{\prime}-1\right)\left(\frac{1}{R_{1}}\right)
$$

\section{b) Modelo lente gruesa}

Cuando el espesor $d$ de la lente es comparable frente a los radios de curvatura de las superficies reflectoras y las distancias s y s' ya no es posible usar el modelo de lente delgada. Para la lente gruesa se consideran los planos principales de la lente. Los planos principales de un sistema de lentes, son dos planos hipotéticos donde se consideran que ocurren todas las refracciones tal como lo indica la Figura 4.5. 


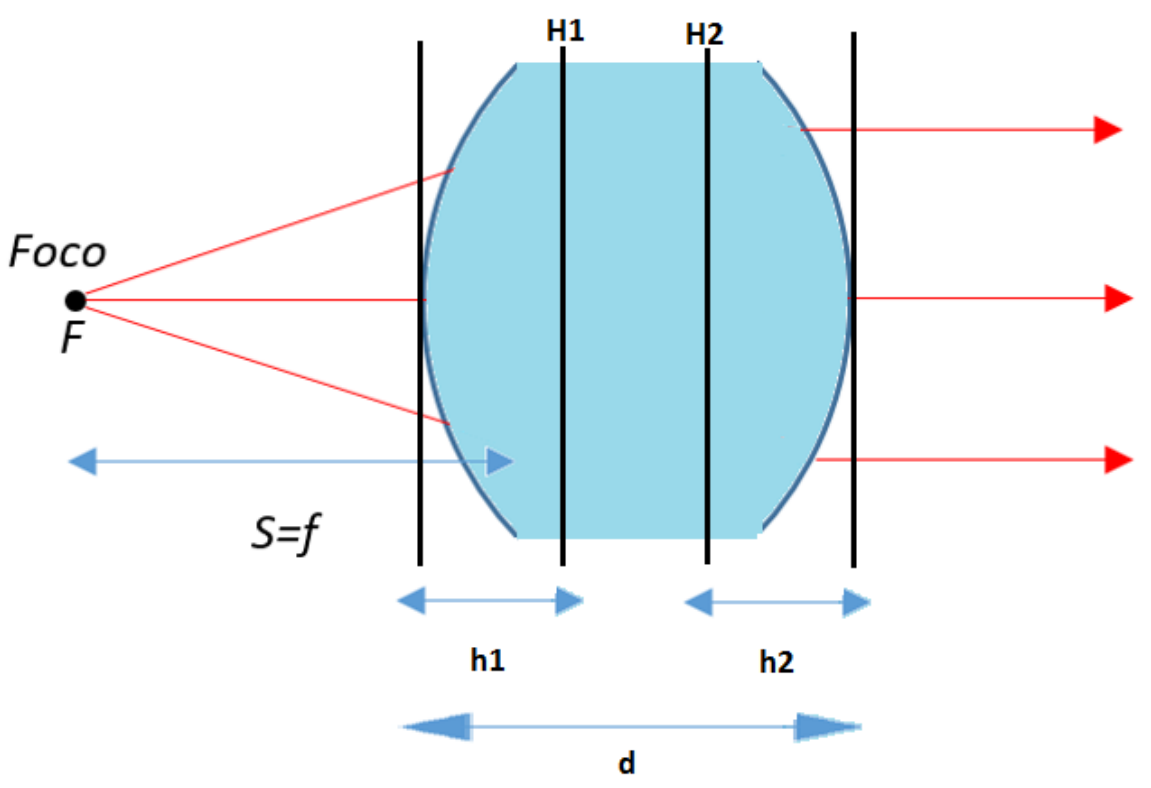

Figura 4.5: Lente esférica gruesa.

Para el diseño de la lente gruesa es posible utilizar la ecuación 4.4 [2].

$$
\frac{1}{f}=\left(n^{\prime}-1\right)\left(\frac{1}{R_{1}}-\frac{1}{R_{2}}+\frac{\left(n^{\prime}-1\right) d}{n^{\prime} R_{1} R_{2}}\right)
$$

Y las distancias de los vértices a los planos principales están dadas por

$$
\begin{aligned}
& h_{1}=-\frac{f\left(n^{\prime}-1\right) d}{n^{\prime} R_{2}} \\
& h_{2}=-\frac{f\left(n^{\prime}-1\right) d}{n^{\prime} R_{1}}
\end{aligned}
$$

\subsection{Aberraciones esféricas}

La aberración esférica es un defecto de los espejos y las lentes en el que los rayos de luz que inciden paralelamente al eje óptico, aunque a cierta distancia de éste, son llevados a un foco diferente que los rayos próximos al mismo. La aberración esférica es una aberración de tipo monocromático de tercer orden que afecta de manera diferente a cada longitud de onda [2]. 


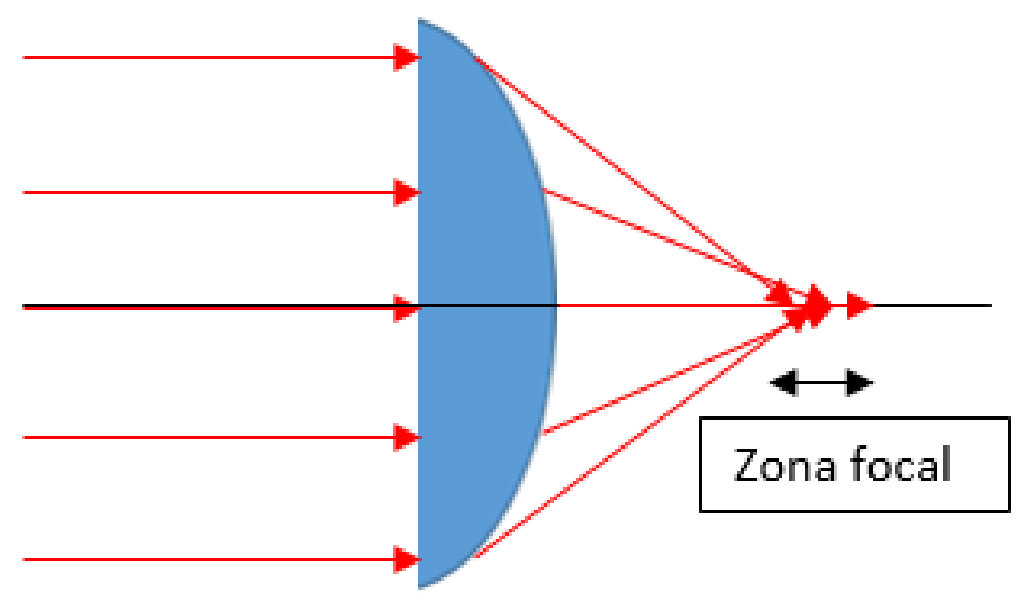

Figura 4.6: Aberración esférica.

Este tipo de aberración provoca que el punto focal sea más difícil de determinar (ya que no toda la energía se concentra en un solo punto) creando una "zona focal". Esto provoca que la eficiencia de la lente se verá disminuida.

\subsubsection{Lentes Asféricas}

Las lentes asféricas son lentes que tienen perfiles cónicos no esféricos como por ejemplo Hiperbólico o Elíptico. Estos perfiles permiten reducir las aberraciones de las lentes esféricas.

\section{a) Lente Elíptica}

La geometría de una lente elíptica puede ser descrita por dos curvas tal como se observa de la Figura 4.6. 


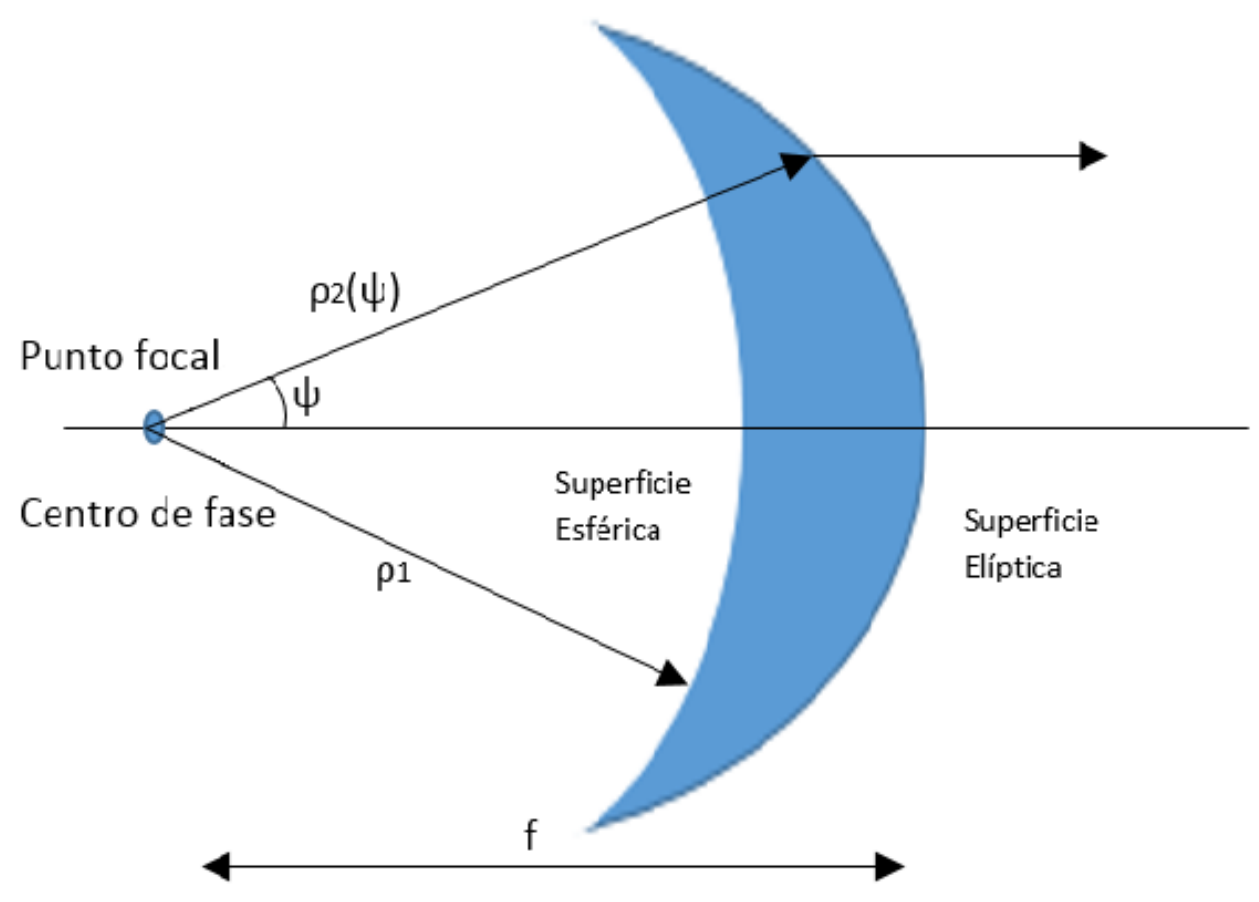

Figura 4.6: Lente Elíptica.

La forma interna (irradiada) de la lente es esférica y se describe por el radio $\rho_{1}$, mientras que la curva exterior es Elíptica y se puede describir en coordenadas polares como $[24,47]$

$$
\rho_{2}(\psi)=\frac{(n-1) f}{n-\cos \psi}
$$

donde $\mathrm{n}$ representa el índice de refracción del material dieléctrico $\left(\varepsilon_{\mathrm{r}}=\mathrm{n}^{2}\right)$ y $\mathrm{f}$ representa la distancia focal de la lente.

\section{b) Lente Hiperbólica Plano convexa}

La ventaja para la utilización de una lente hiperbólica consiste principalmente en su forma simple y por lo tanto más fácil de construir. Los parámetros de la lente Hiperbólica se pueden observar en la Figura 4.7. 


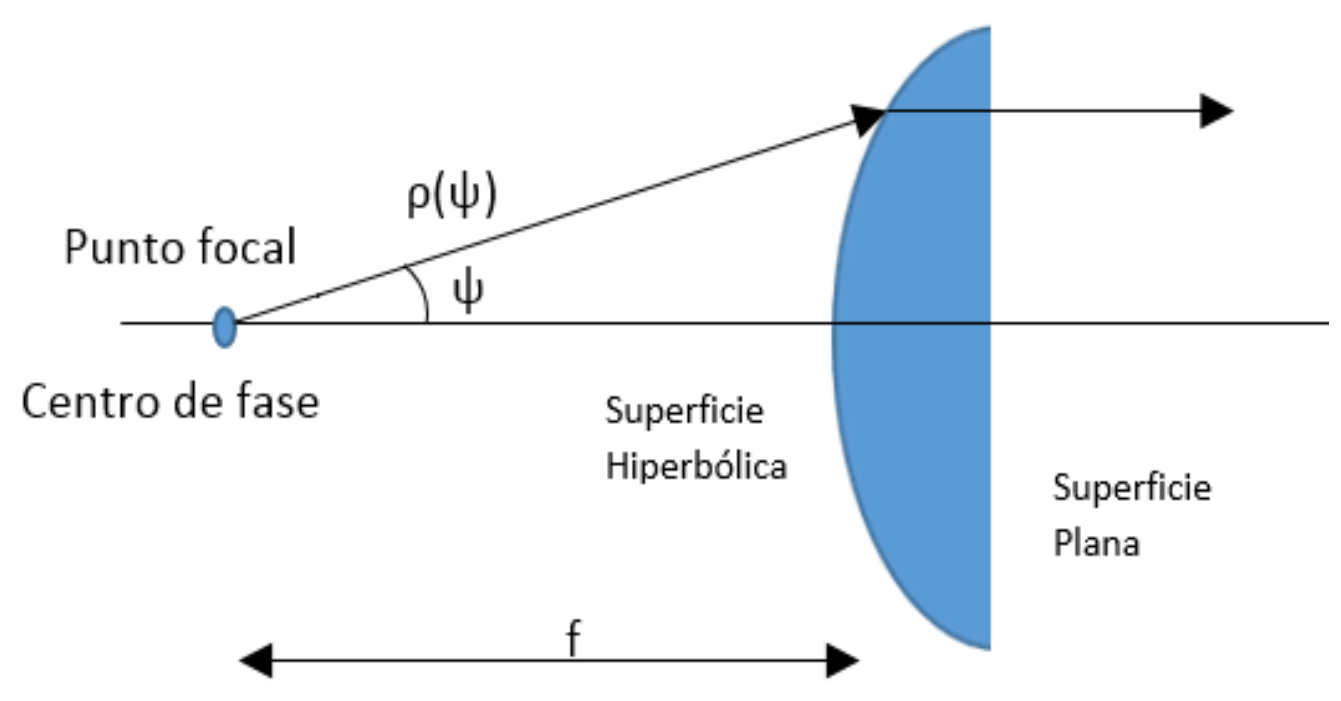

Figura 4.7: Lente Hiperbólica plano convexa.

La cara Hiperbólica se puede expresar en coordenadas polares mediante [24, 47]

$$
\rho(\psi)=\frac{(n-1) f}{n \cos \psi-1}
$$

\section{c) Lentes Perfectas}

Si se desea diseñar una lente ideal de forma que cada punto a la salida de la lente tenga igual fase (condición de onda plana) para lograr la máxima directividad de la antena lente, puede plantearse dicha condición y verificar qué curva arroja la lente.

Desde el punto de la Óptica Geométrica deberá plantearse que cada "rayo" recorra el mismo camino óptico, tal como se observa en la Figura 4.8. 


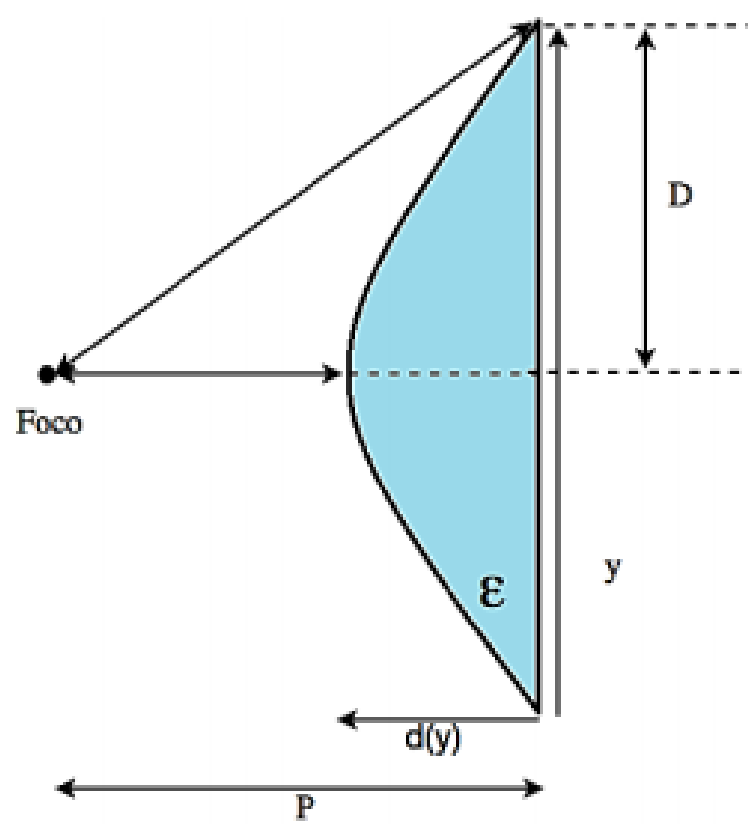

Figura 4.8: Lente "perfecta".

Para comenzar el diseño se mantiene fijo el radio D de la lente. Esta lente basa la igualación de camino en función de su espesor respecto al eje y.

Si se desea que el rayo que incide en la parte superior de la lente y cualquier otro que la atraviese, arribe al mismo tiempo a la salida de la lente, se puede plantear una ecuación donde se iguale el tiempo de arribo de ambos rayos.

En los bordes la onda incide y se desvía sin llegar a entrar a la lente, solo la toca, por lo que el tiempo que se tarda desde el foco hasta ese extremo es

$$
t_{\text {borde }}=\frac{s}{v}=\frac{\sqrt{P^{2}+D^{2}}}{c_{0}}
$$

donde $s$ es la distancia recorrida, $v$ la velocidad, y $c_{0}$ la velocidad de la luz en el vacío.

En el resto de la lente los rayos inciden y son retardados debido a que el material (dieléctrico) tiene una permitividad eléctrica $\varepsilon$ mayor que en el exterior (aire). El tiempo que la señal tarda en atravesar cualquier parte de la lente desde la fuente está dado por el tiempo que tarda en atravesar el aire más el tiempo que tarda en atravesar la lente.

$$
t_{\text {lente }}=t_{\text {aire }}+t_{\text {material }}=\frac{\sqrt{y^{2}+(P-d(y))^{2}}}{c_{0}}+\frac{d(y)}{c_{0}} \sqrt{\varepsilon}
$$

Se desea que el tiempo total de viaje de la señal que llega a los extremos, iguale al tiempo total de la señal que viaja por el eje, parte por el aire y parte atravesando la lente 
(igualdad de fase a la salida de la lente), por lo que las fórmulas 4.9 y 4.10 deben ser igualadas, $t_{\text {borde }}=t_{\text {lente }}$.

$$
\frac{\sqrt{P^{2}+D^{2}}}{c_{0}}=\frac{\sqrt{y^{2}+(P-d(y))^{2}}}{c_{0}}+\frac{d(y)}{c_{0}} \sqrt{\varepsilon}
$$

y, además, despejando la velocidad y considerando que

$$
T=\sqrt{P^{2}+D^{2}}
$$

se puede resolver la ecuación 4.11. Ordenando términos se llega a la siguiente ecuación de segundo grado

$$
d(y)^{2}(\varepsilon-1)+d(y) \cdot(2 P-2 T \sqrt{\varepsilon})+T^{2}-y^{2}-P^{2}=0
$$

La ecuación 4.13 describe una Hipérbola. Es decir, la lente hiperbólica es el caso óptimo para reducir las aberraciones esféricas.

\section{d) Lente escalonada de Fresnel}

En muchas aplicaciones, especialmente en microondas donde la longitud de onda es mayor que en aplicaciones ópticas, el tamaño de una lente convencional de los perfiles analizados puede resultar considerablemente grande. En estos casos se podría utilizar una lente de Fresnel, con el fin de disminuir su espesor y con ello disminuir el peso de la misma.

Las llamadas lentes de Fresnel, surgen de una modificación del diseño de la lente hiperbólica plano-convexa, tendiente a ahorrar la cantidad de material dieléctrico a emplear, resultando sensiblemente más livianas. Es por ello que han sido tradicionalmente utilizadas cuando la reducción de peso es importante, como en los faros, retroproyectores, etc.

El diseño de una lente de Fresnel puede obtenerse a partir de una lente hiperbólica. Una vez creada la lente de perfil hiperbólico se puede reducir el tamaño de la lente eliminando de forma escalonada cilindros de material de espesor $\Delta$ de modo que la diferencia de caminos eléctricos en cada escalón sea un número entero de longitudes de onda [61].

$$
\Delta n-\Delta=m \lambda_{0} \quad m=1,2,3 \ldots .
$$




$$
\Delta=m \frac{\lambda_{0}}{n-1} \quad \mathrm{~m}=1,2,3 \ldots
$$

Siendo $n$ el índice de refracción del dieléctrico de la lente $\left(\varepsilon_{\mathrm{r}}=\mathrm{n}^{2}\right)$ y $\lambda_{0}$ la longitud de onda para la cual se diseña la lente.
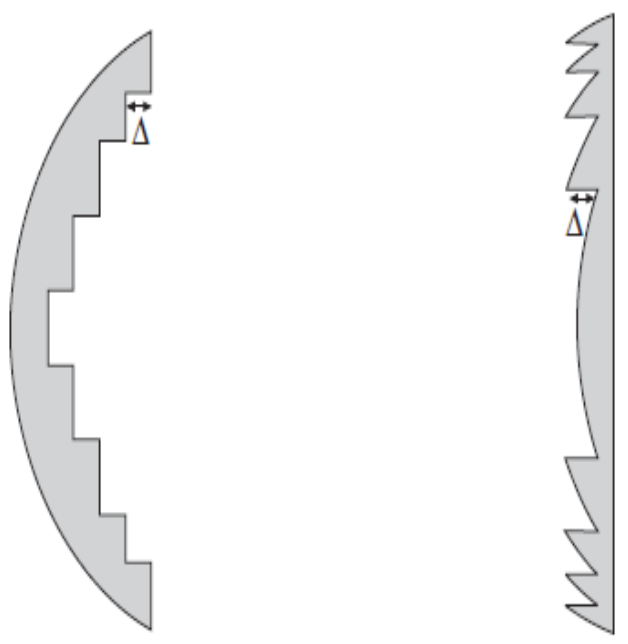

Figura 4.9: Diseño de lente escalonada de Fresnel.

Un esquema de la antena lente es mostrado en la Figura 4.10. Dicha antena lente es diseñada para la frecuencia central $\mathrm{f}_{0}$ del alimentador principal (Fuente).

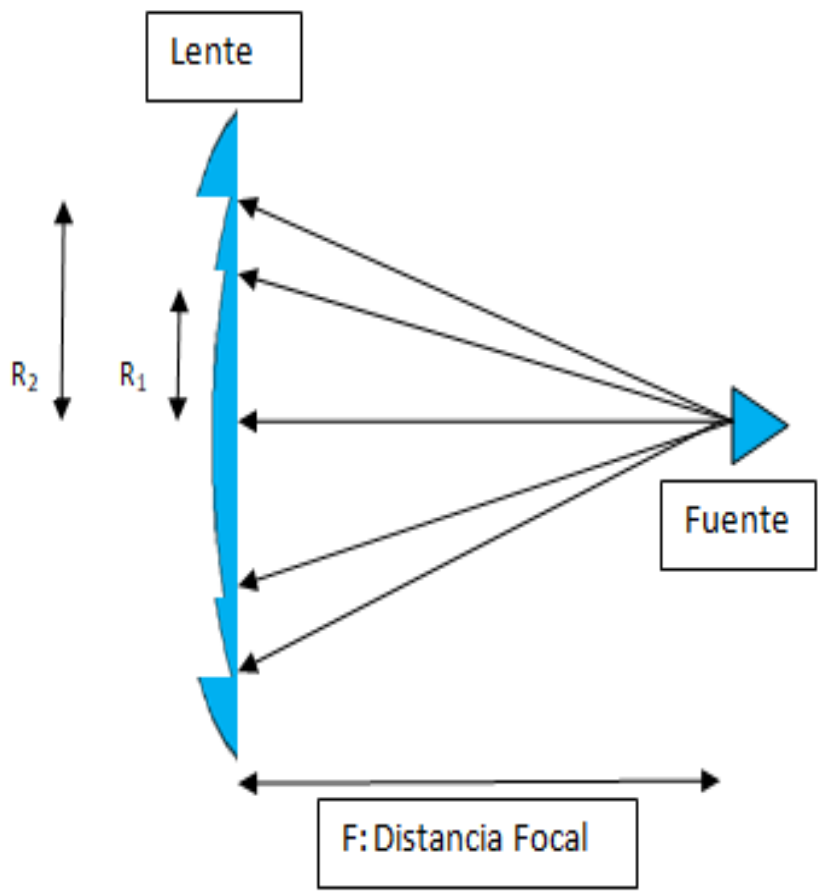

Figura 4.10: Lente escalonada de Fresnel. 
Dado que el diseño de la lente de Fresnel se realiza para una dada frecuencia, al variar la frecuencia del alimentador los caminos eléctricos no diferirán en múltiplos exactos de la cada longitud de onda.

Si existen $\mathrm{N}$ zonas a partir del centro de la lente, la diferencia máxima de caminos al variar la frecuencia será:

$$
\delta=(\mathrm{N}-1)(\Delta \lambda)
$$

Si se toma como criterio $\delta=\lambda_{0} / 8[61]$, se obtiene que

$$
\frac{\Delta \lambda}{\lambda_{0}}=\frac{1}{8}(\mathrm{~N}-1)
$$

Por lo tanto, el ancho de banda relativo (BW) para la antena lente estará dado por la siguiente ecuación, [61]:

$$
\mathrm{BW}=2 \frac{\Delta \lambda}{\lambda_{0}}=\frac{0,25}{\mathrm{~N}-1}
$$

\section{e) Lente de Luneberg}

La lente de Luneberg es una lente esférica que tiene la propiedad de concentrar los rayos de una onda plana incidente en un punto de su superficie.

El punto donde se concentran los rayos está diametralmente opuesto a la dirección de incidencia. Como la esfera tiene simetría de revolución, la propiedad se cumple con independencia de la dirección de incidencia. 


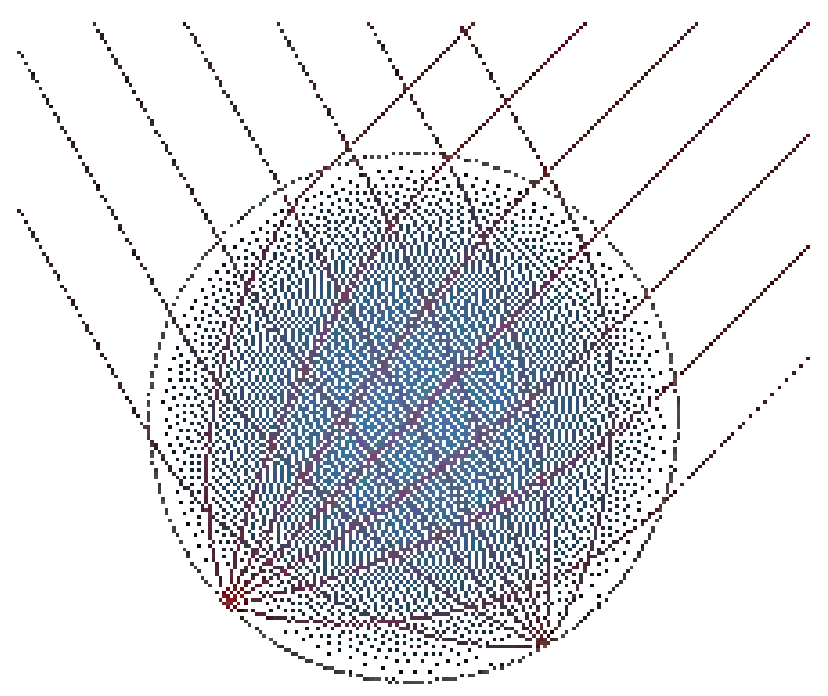

Figura 4.11: Lente de Luneberg.

La lente tiene un índice de refracción variable dada por

$$
n=\sqrt{2-r^{2} / r_{0}^{2}}
$$

donde $\mathrm{r}_{0}$ es el radio de la esfera y $\mathrm{r}$ es la distancia desde el centro. En el centro el índice de refracción vale $\sqrt{2}$ y disminuye gradualmente hasta la periferia, donde vale 1 .

En óptica es difícil conseguir este control de los materiales, pero a frecuencias de microondas es más fácil, y de hecho se utilizan lentes de Luneberg modificadas como reflectores retrodirectivos para radar. Para ello se recubre la semiesfera posterior con un material reflectante (aluminio o cobre, por ejemplo), y así la onda incidente y reflejada tienen la misma dirección [2]. 


\section{CAPÍTULO 5}

\section{Diseño de antenas lente dieléctricas en Microondas mediante método full-wave}

\subsection{Introducción}

Como se vio en el Capítulo 4, el diseño de lentes clásicas ha sido ampliamente estudiado. Las ecuaciones que describen los perfiles de las lentes clásicas derivan mayoritariamente de la Óptica Geométrica, que parte de las leyes fenomenológicas de Snell de la reflexión y la refracción. Como se repasó en el Capítulo 2, la Óptica Geométrica usa la noción de rayo luminoso; siendo una aproximación del comportamiento que corresponde a las ondas electromagnéticas (la luz) cuando los objetos involucrados son de tamaño mucho mayor que la longitud de onda usada; ello permite despreciar los efectos derivados de la difracción, comportamiento ligado a la naturaleza ondulatoria de la luz.

Para el diseño de antenas lentes en microondas ya no es posible utilizar métodos asintóticos sin errores considerables por lo que es deseable contar con métodos full-wave que arrojen resultados más precisos.

Por otro lado, el diseño de una antena en general viene determinado por las características eléctricas deseadas (ganancia, ancho de haz principal, relación lóbulo principal a secundario, relación front to back, etc). Dependiendo del tipo de antena, existen varios parámetros que se pueden variar para lograr dichas características.

En particular, las antenas lente cuentan con dos elementos principales. El alimentador, que puede ser una bocina, y una lente como se estudiaron en el Capítulo 4. Dependiendo del problema en particular se puede variar las características tanto del alimentador como de la lente.

En el presente Capítulo se analizan y proponen métodos de diseño de lentes mediante simulación computacional con métodos full-wave. Para ello se utilizó el código de simulación full-wave estudiado en el Capítulo 2. Todos los resultados expuestos en el presente Capítulo son originales. 


\subsection{Parámetros de diseño de las antenas lente}

Existen diferentes parámetros que definen una antena. La importancia de cada parámetro depende de los requisitos de la aplicación.

Los parámetros a tener en cuenta de la lente son los siguientes:

a) Frecuencia de operación.

b) Diámetro (Determina la ganancia).

c) Distancia focal.

d) Tipo de material (Determina el espesor de la lente, perdidas por reflexión y las perdidas por transmisión).

e) Perfil de la lente (depende de los parámetros anteriores).

Los pasos del diseño pueden variar según los siguientes tipos de problemas

1. Se desea diseñar una lente para mejorar las características eléctricas de un alimentador dado.

2. Se desea diseñar para una lente dada, el alimentador óptimo para dicha lente.

En general una vez determinados los parámetros requeridos para el alimentador y la lente, se procede al diseño del conjunto antena lente. Como los parámetros tanto del alimentador como de la lente están interrelacionados, por lo general se comienza con un diseño y luego se sigue un proceso iterativo de modo de ajustar las características deseadas. La Figura 5.1 muestra un diagrama de diseño de la antena lente.

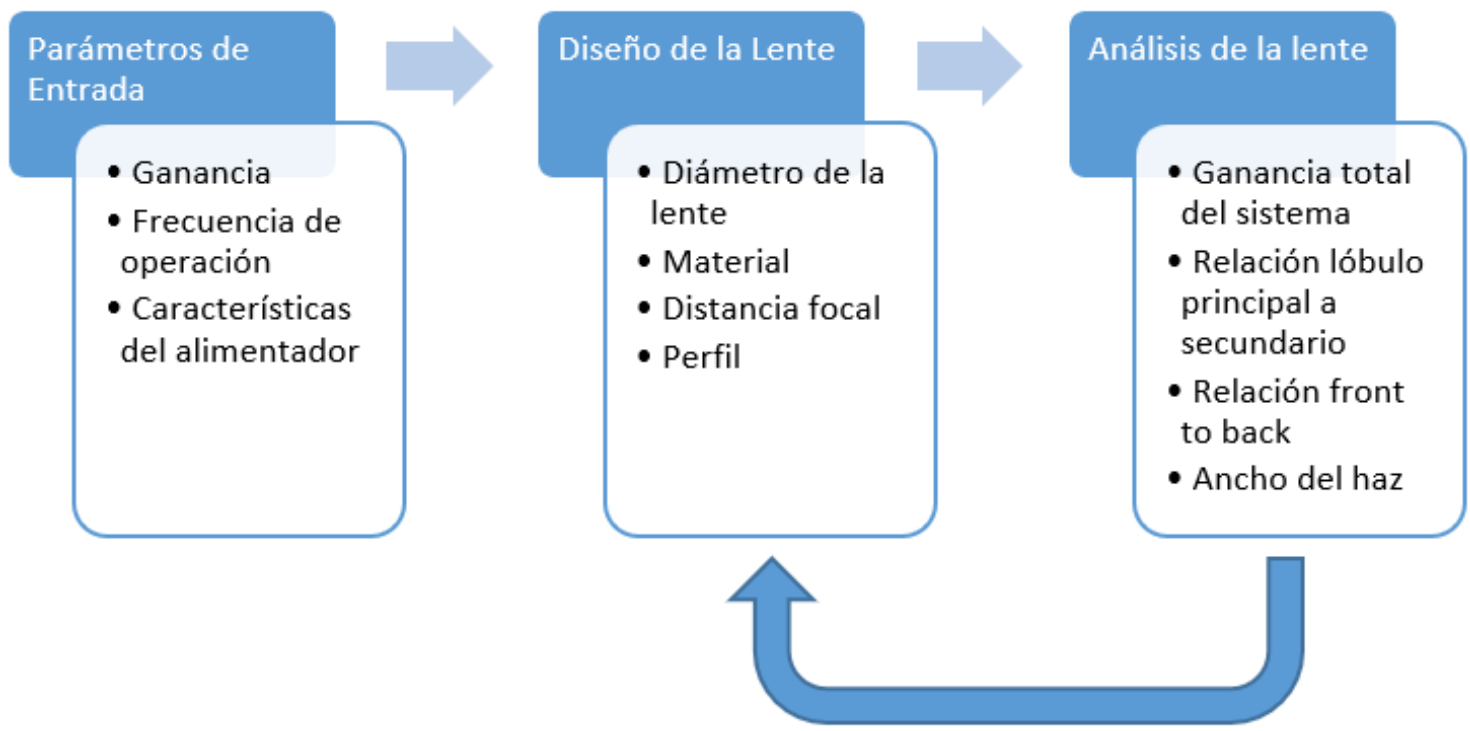

Figura 5.1: Proceso de diseño de antenas lente. 


\subsubsection{Procedimiento para el diseño de antenas lentes}

El procedimiento para el diseño de antenas puede variar según la aplicación, pero en general se pueden seguir los siguientes pasos:

\section{a) Frecuencia de operación}

En el caso donde se desee diseñar una lente para un alimentador, como puede ser una bocina, la frecuencia de operación viene determinada por la aplicación y en particular por el alimentador.

\section{b) Ganancia}

El segundo paso consiste en evaluar la característica más relevante de la antena como puede ser la Ganancia o la relación lóbulo principal a secundario.

Si se desea lograr una determinada ganancia, se puede emplear la ecuación 3.22 del Capítulo 3. El diámetro de la lente en función de la longitud de onda $(\lambda)$ permite fijar una ganancia teórica requerida.

\section{c) Eficiencia y relación $f / D$}

Luego para obtener la máxima eficiencia del conjunto antena lente, tal como se detalla en el inciso 3.3.3 del Capítulo 3, es importante determinar la distancia focal para lograr la relación $f / D$ que maximice la eficiencia de spillover e Iluminación.

Como se vio en el punto 3.3.5 del Capítulo 3, se encuentra en general que, para iluminaciones en los bordes del orden de $-10 \mathrm{~dB}$ respecto al centro, se suelen obtener los mejores diseños en cuanto a directividad, mientras que en torno a $-20 \mathrm{~dB}$ es el nivel habitual para una buena relación lóbulo principal a secundario (NLPS).

En aplicaciones donde sea posible diseñar la lente junto a su alimentador se puede comenzar diseñando la lente y luego el alimentador que se adapte mejor a dicha lente teniendo en cuenta los conceptos de máximo rendimiento. Generalmente este tipo de diseño resulta cuando por cuestiones eléctricas o mecánicas (tamaño de la lente), las dimensiones y/o material de la lente no pueden ser modificadas. 


\section{d) Material dieléctrico de la Lente}

El material dieléctrico con el que se construya la lente es determinante en el perfil de la lente. Mientras mayor constante dieléctrica del material $\left(\varepsilon_{\mathrm{r}}\right)$ más delgada resultará la lente (con lo cual disminuirá su peso), pero mayores pérdidas por transmisión tendrán.

El código de simulación 2D utilizado no computa las perdidas por transmisión (perdidas del material), pero se puede obtener un valor aproximado de estas pérdidas utilizando la ecuación 3.27 del Capítulo 3 de antenas de apertura.

\section{e) Elección del perfil de la lente}

Una vez determinada la distancia focal se debe diseñar el perfil de la lente cuyo foco coincida con la distancia focal calculada. En ese punto se debe colocar el centro de fase del alimentador. Los diferentes perfiles se analizan posteriormente en el presente Capítulo.

\subsection{Determinación del foco de una lente}

La distancia focal y precisamente el punto donde se encuentra el foco de la lente, son primordiales en el desempeño del conjunto antena lente. Debido a que el centro de fase del alimentador primario debe ir en dicho foco, es fundamental conocer la posición exacta de éste.

La determinación del foco teórico de las lentes a partir de las ecuaciones analíticas vistas en el Capítulo 4, obtenidas en general de la aplicación de métodos aproximados, tales como el trazado de rayos, no siempre da resultados que permitan determinar su posición con buena precisión.

Como se analizó en el Capítulo 2, mientras las dimensiones globales de la lente y el radio de curvatura de la superficie en cualquier punto sean mucho más grandes que la longitud de onda, la propagación de la onda dentro de una lente isotrópica homogénea puede modelarse convenientemente en términos de tubos de rayos elementales. Aunque la síntesis por Óptica Geométrica es suficiente para muchas aplicaciones, el método es asintótico, válido en el límite óptico, por lo que se descuidan los efectos de difracción que se vuelven importantes a medida que se reduce el tamaño de la lente. En frecuencias como las microondas, donde el tamaño de la lente es comparable a la longitud de onda, los métodos como GO pueden ser inaceptables. Las simulaciones electromagnéticas de onda completa (full-wave) proporcionan resultados más precisos.

Para demostrar esta situación se propone el diseño de dos lentes, una de perfil esférico y otra de perfil hiperbólico mediante las ecuaciones 4.4 (lente gruesa) y 4.13 respectivamente utilizando los parámetros de la Tabla 5.1. 
Tabla 5.1: Características de las antenas lentes diseñadas.

\begin{tabular}{|c|c|c|c|c|}
\hline Lente & $\begin{array}{c}\text { Diámetro } \\
\text { [mm] }\end{array}$ & $\begin{array}{c}\text { Permitividad } \\
\text { relativa }\end{array}$ & $\begin{array}{c}\boldsymbol{R}_{\boldsymbol{I}}=\boldsymbol{R}_{\mathbf{2}} \\
{[\mathbf{m m}]}\end{array}$ & $\begin{array}{c}\text { Espesor } \\
\boldsymbol{d} \text { [mm] }\end{array}$ \\
\hline Hiperbólica & 300 & 4 & - & - \\
\hline Biconvexa & 300 & 4 & 300 & 120 \\
\hline
\end{tabular}

Para determinar el punto focal de ambas lentes con alta exactitud, se simulan ambas lentes en recepción mediante el código de simulación full-wave estudiado en el Capítulo 2. Se utilizó la versión $2 \mathrm{D}$ del código ya que las lentes tienen simetría de revolución por lo que los resultados no cambian en 3D [68].

Para ello se irradian las lentes con una onda plana, tal como se observa en la Figura 5.2, y se analizan los diagramas del módulo de campo eléctrico, lo que permite determinar la distancia focal.

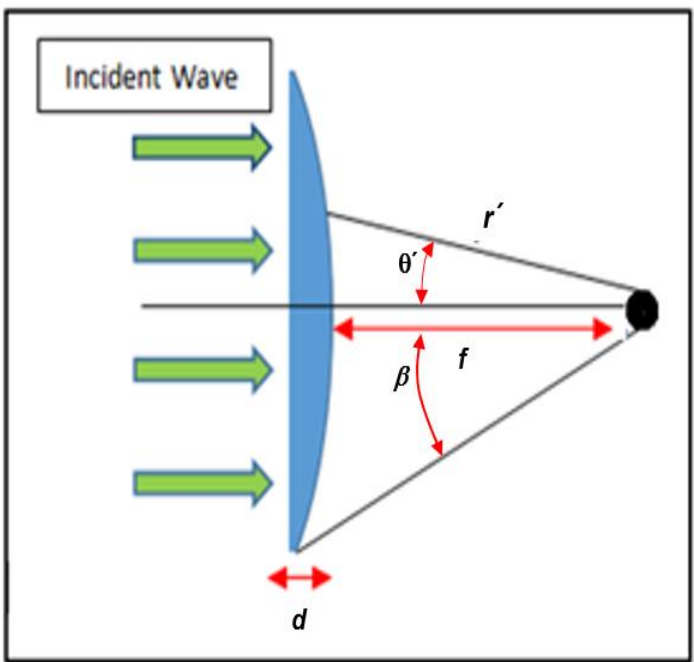

a)

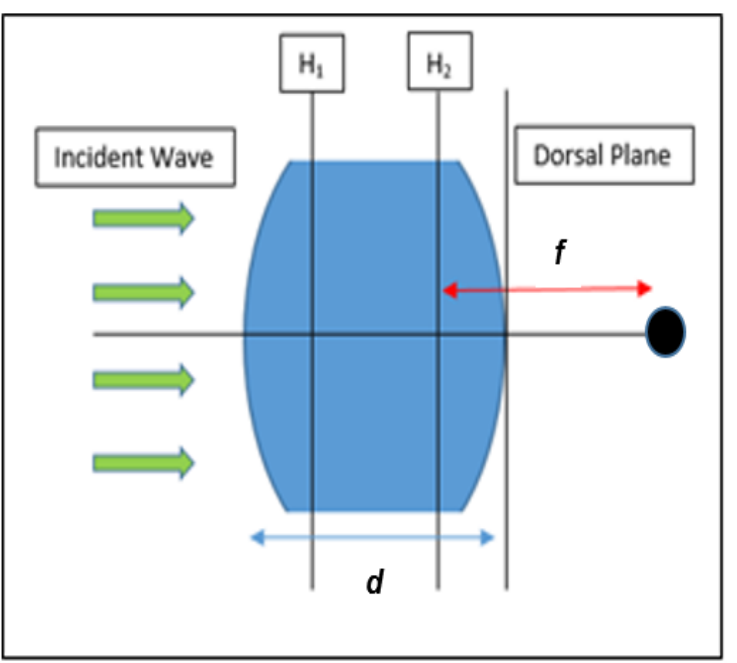

b)

Figura 5.2: Esquema de simulación para determinación de la distancia focal de la lente a) Hiperbólica y b) Esférica biconvexa.

Las simulaciones se realizan a una frecuencia de $30 \mathrm{GHz}(\lambda=1 \mathrm{~cm})$.

En la Figura 5.3 a) y b), se muestran las simulaciones de los diagramas del módulo de campo electromagnético fuera y dentro de las lentes estudiadas. En dichas figuras se puede observar una mayor intensidad del campo electromagnético en el área donde se encuentra el foco de las lentes (el color rojo corresponde a las intensidades más altas). 


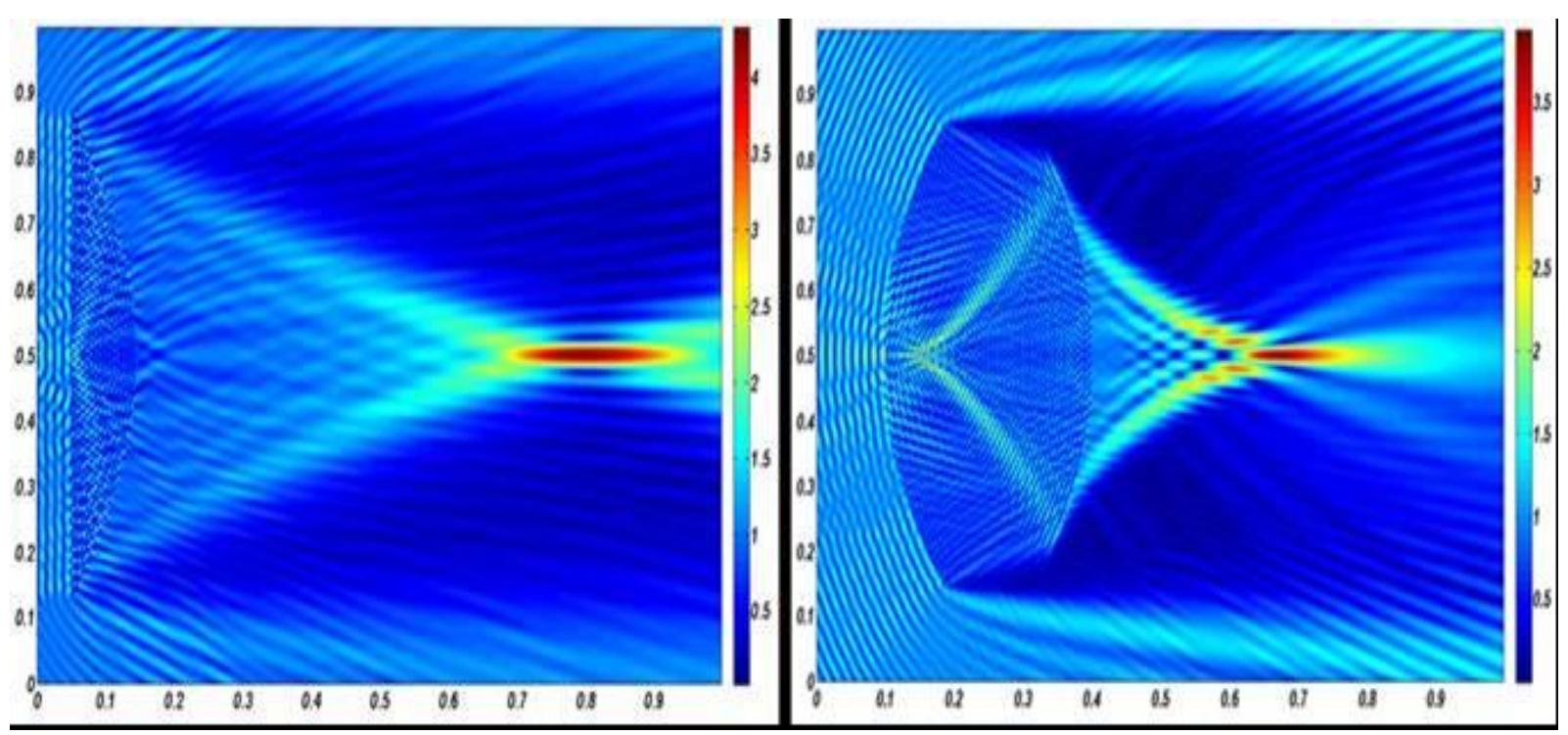

a)

b)

Figura 5.3: Módulo del campo eléctrico cercano simulado para la antena lente a) Hiperbólica y b) Esférica biconvexa. Los ejes indican las dimensiones del cuadrado unidad.

De la Figura 5.3 b) (Lente Esférica) es posible observar como la energía en la zona focal no se concentra en un solo punto, sino que se dispersa en una zona. Esto se debe a la aberración esférica vista en el Capítulo 4 inciso 4.2.1.1.1.

La distancia focal se determina realizando un análisis de las intensidades focales en el eje de la lente en los diagramas del módulo de campo eléctrico en ambas lentes. Luego, la ubicación del foco será el punto de máxima intensidad de las gráficas del perfil como se ve en la Figura 5.4 a) y b). En el eje de las abscisas se indican los nodos (N) utilizados en la simulación. Se utilizaron diez puntos por longitud de onda, por lo que la

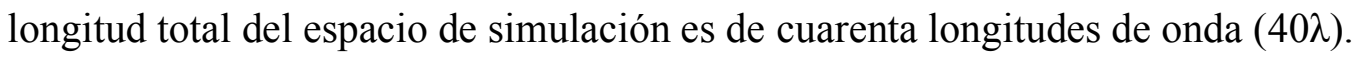




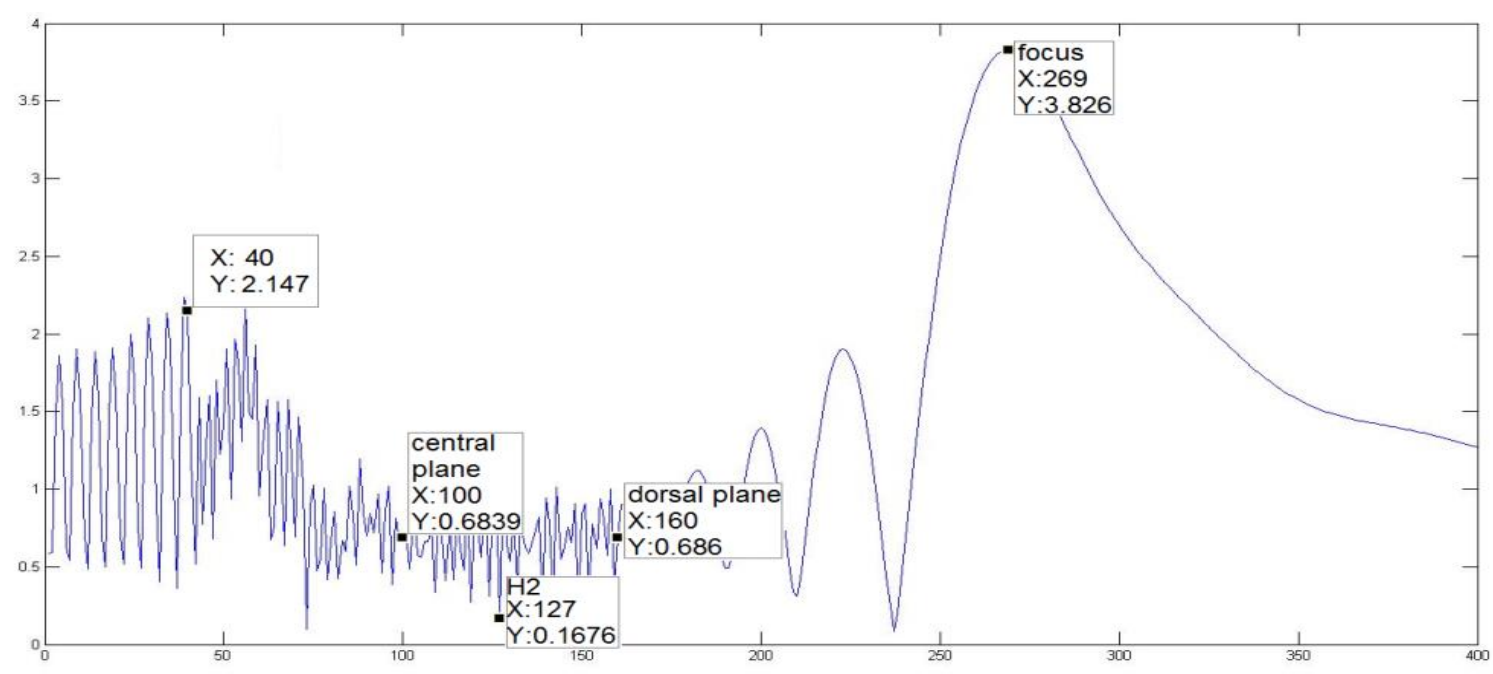

a)

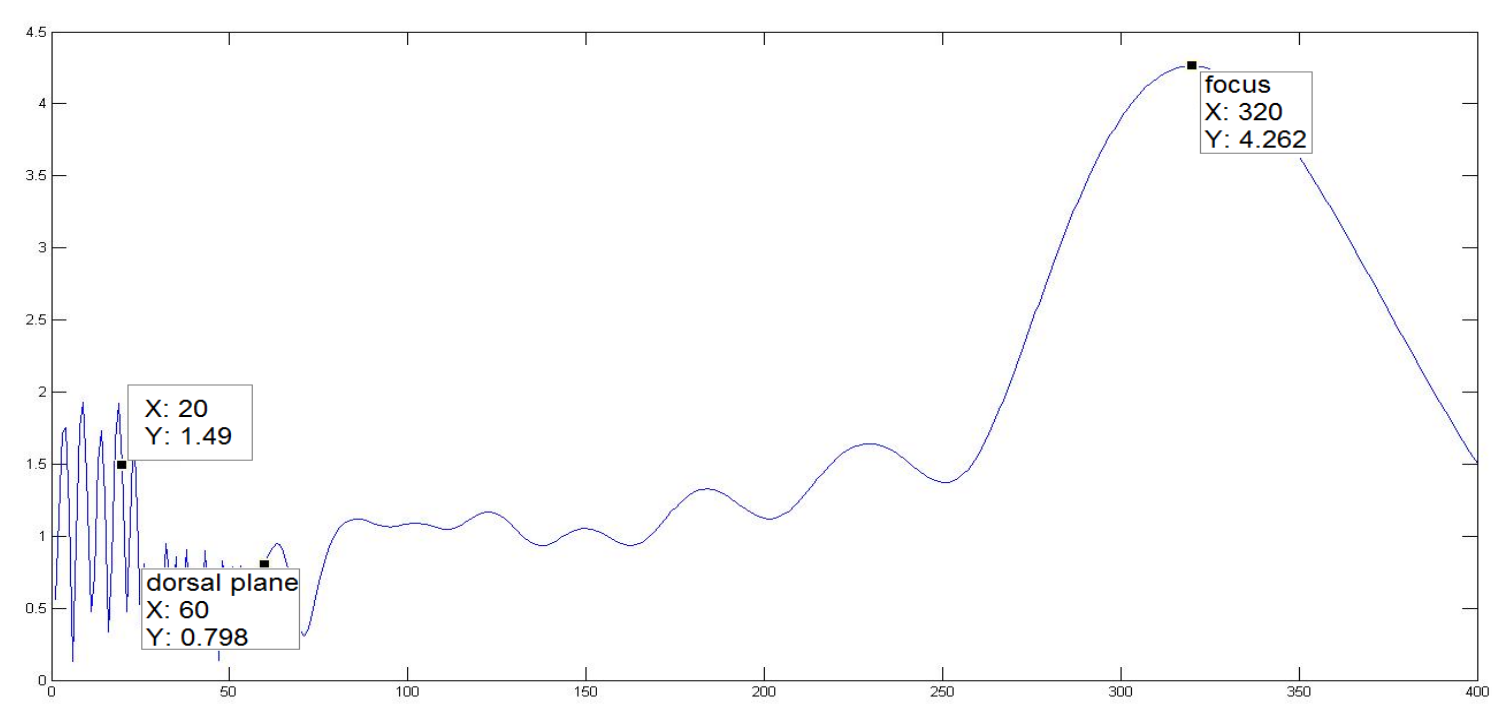

b)

Figura 5.4: Módulo del campo eléctrico cercano simulado en el eje de la lente a) Hiperbólica y b) Esférica biconvexa.

En la Tabla 5.2. se vuelcan las distancias focales obtenidas por simulación junto a los valores obtenidos según los cálculos teóricos de las ecuaciones derivadas de la Óptica Geométrica. En la misma tabla se indican los tiempos de cálculo utilizados en la simulación para cada una de las lentes.

La distancia focal teórica de la lente hiperbólica observada en la Tabla 5.2 es el resultado de aplicar la ecuación 4.8, mientras que la longitud focal simulada se obtuvo a partir de la gráfica del perfil del campo eléctrico en el eje focal de la lente (Figura 5.3 a). Para la lente biconvexa, la distancia focal teórica responde a 4.4, mientras que la distancia focal simulada se obtuvo de la Figura 5.3 b). 
Tabla 5.2: Distancia focal de las lentes simuladas.

\begin{tabular}{|c|c|c|c|}
\hline \multirow{2}{*}{$\begin{array}{c}\text { Antena } \\
\text { Lente }\end{array}$} & \multicolumn{2}{|c|}{ Distancia focal [mm] } & \multirow{2}{*}{$\begin{array}{c}\text { Tiempo } \\
\text { computacional } \\
{[\mathrm{s}]}\end{array}$} \\
\hline & Teórica & Simulada & \\
\hline Hiperbólica & 260 & 260 & 208 \\
\hline Biconvexa & 166 & 142,3 & 216 \\
\hline
\end{tabular}

Vale la pena señalar que la determinación teórica de la distancia focal no depende del tamaño de la lente (las ecuaciones 4.4 y 4.8 no dependen del diámetro de la lente). Mediante la simulación se obtiene una determinación más precisa de la distancia focal, dependiendo del tamaño de la lente en función de la longitud de onda, ya que considera los efectos electromagnéticos que ocurren en el sistema, como la difracción en los bordes.

La determinación del foco de la lente según los cálculos teóricos difiere de la obtenida por simulación para el caso de la antena biconvexa. Esta diferencia se debe al hecho de que el modelo teórico aplica la Óptica Geométrica, trazado de rayos, mientras que la simulación electromagnética se ocupa del análisis de onda completa. Esto es particularmente importante en el presente caso de estudio, donde las lentes son comparables a la longitud de onda en la región de microondas.

En el caso de la antena de lente hiperbólica, los resultados son coincidentes, ya que el propio diseño de la lente (Capítulo 4, inciso 4.2.1.2 c) lente perfecta), con sus ecuaciones correspondientes, tiene en cuenta el fenómeno de dispersión electromagnética.

También es posible determinar el punto focal de la lente a partir del diagrama de fase. A modo de ejemplo se propone simular una lente elíptica.

La lente está diseñada por un material con una permitividad dieléctrica igual a $\varepsilon_{\mathrm{r}}$ $=3,78$. El espacio de simulación (cuadrado unidad) tiene dimensiones de $40 \lambda \times 40 \lambda$ (por lo que cada longitud de onda representa $\lambda=1 / 40=0,025$ partes de la unidad). Para obtener una buena resolución se simuló con $\mathrm{N}=400$ puntos, de modo de tener 10 puntos por unidad longitud de onda.

El diámetro de la lente es de $30 \mathrm{~cm}(30 \lambda)$ y la distancia focal es de $30 \mathrm{~cm}$. Con lo cual la relación $\mathrm{f} / \mathrm{D}=1$.

La Figura 5.5 a) y b) muestra el diagrama del módulo y la fase del campo dentro y fuera de la lente respectivamente. 


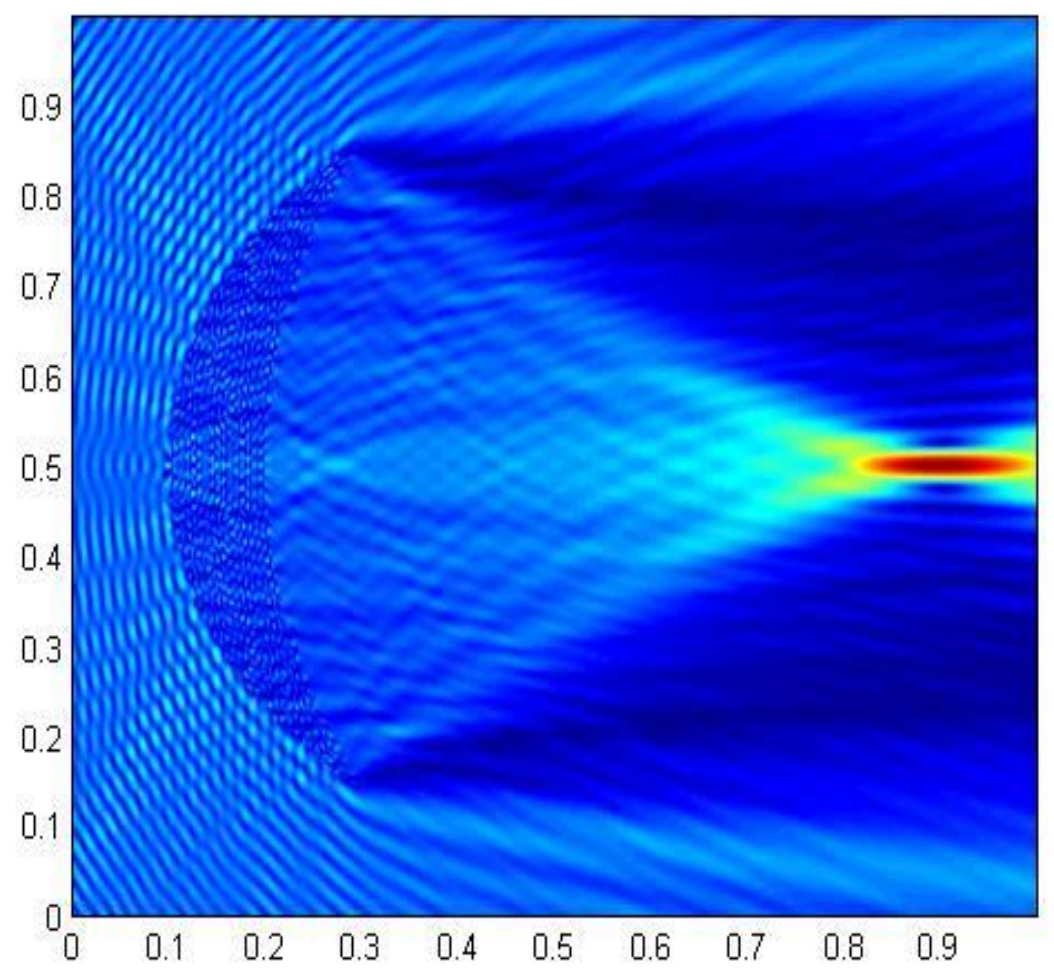

a)

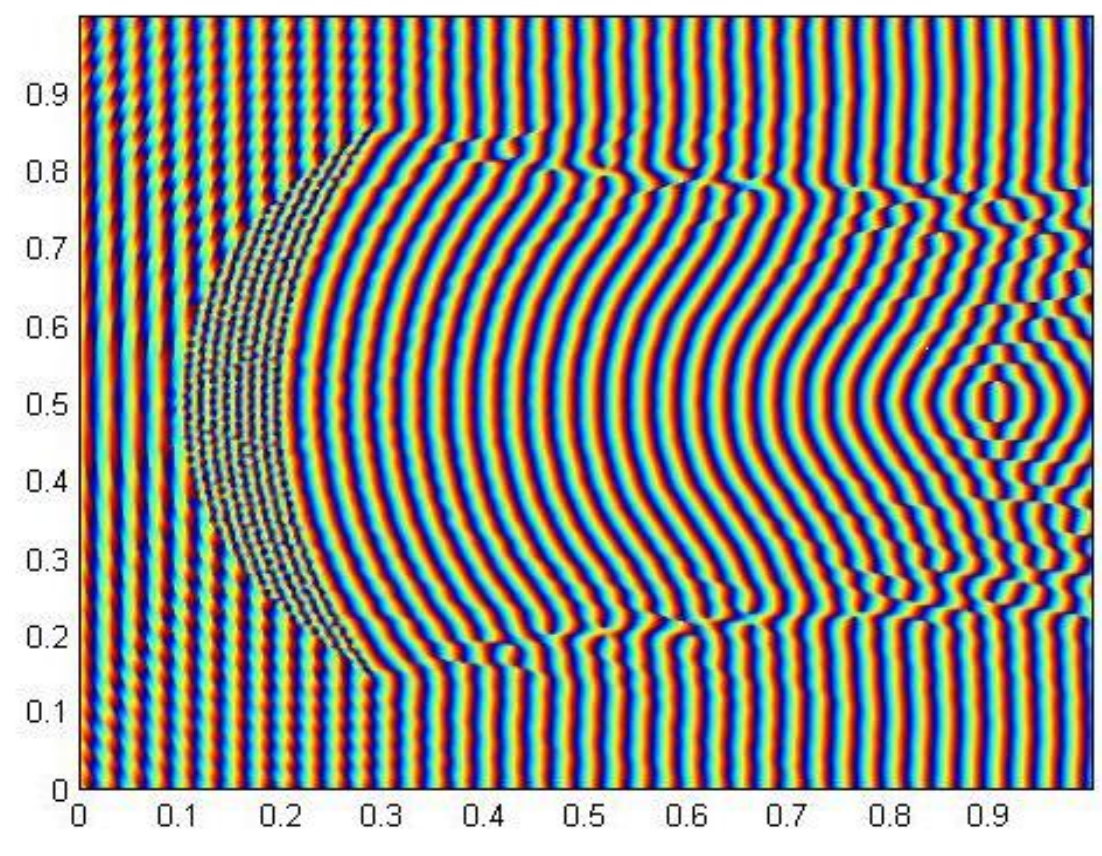

b)

Figura 5.5: Simulaciones de a) Modulo y b) Fase del campo eléctrico cercano para la lente elíptica. Los ejes indican las dimensiones del cuadrado unidad. 
Del diagrama de fase se puede observar cómo incide la onda plana (Fase constante) y luego la lente modifica la fase para que se genere una onda esférica con centro de fase en el punto focal. Se observa también como la onda plana incidente no tiene fase perfectamente constante, esto se debe a las reflexiones que se producen en la lente y se superponen a la onda incidente.

Las simulaciones han demostrado la importancia de contar con herramientas de simulación electromagnética con métodos de onda completa para obtener los valores del campo eléctrico de la antena lente que permiten determinar la distancia focal con mayor precisión, particularmente para aplicaciones en microondas [69]. Además, como se puede ver en la Tabla 5.2, las simulaciones con el código utilizado permiten obtener resultados en muy poco tiempo.

\subsection{Análisis de diferentes perfiles de lentes}

\subsubsection{Simulaciones de lentes de perfiles cónicos}

Con el fin de analizar diferentes perfiles cónicos de lentes mediante el código de simulación full-wave 2D estudiado, se propone el diseño y simulación de dos antenas lente, una de perfil hiperbólico y la otra elíptico, con igual diámetro y permitividad eléctrica, con el fin de poder comparar sus características como antenas [70, 71].

La frecuencia central del diseño es de $30 \mathrm{GHz}(\lambda=1 \mathrm{~cm})$. El diámetro de diseño de las lentes es de $\mathrm{D}=30 \mathrm{~cm}(30 \lambda)$ y la permitividad eléctrica relativa $\varepsilon_{\mathrm{r}}=4$.

En primera instancia se determina el punto focal para cada lente, donde se debe ubicar el alimentador del sistema. Para ello se simula la incidencia de una onda plana a la frecuencia central y se mide la distancia focal tal como se vio en el inciso 5.3.

Para la simulación se utiliza una fuente del tipo cardiode cuyo diagrama de radiación está dado por la siguiente expresión,

$$
S(\varphi)=\left(\frac{1+\cos (\varphi)}{2}\right)^{q}
$$

El valor de q se determina con el fin de lograr la máxima eficiencia (eficiencia de iluminación y spillover).

La Figura 5.6 muestra en una escala de intensidad de color (rojo mayor intensidad, azul menor intensidad) el módulo del campo dentro y fuera de la lente para el caso de la antena lente hiperbólica (a) y la elíptica (b). 


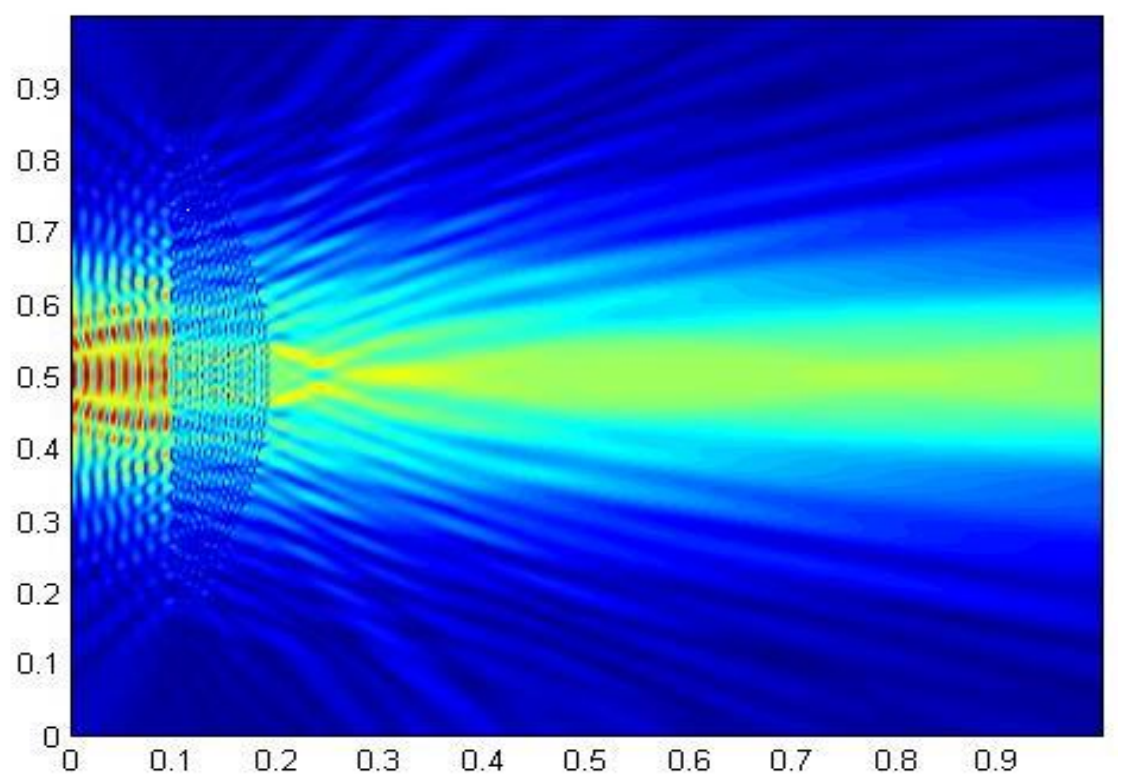

a)

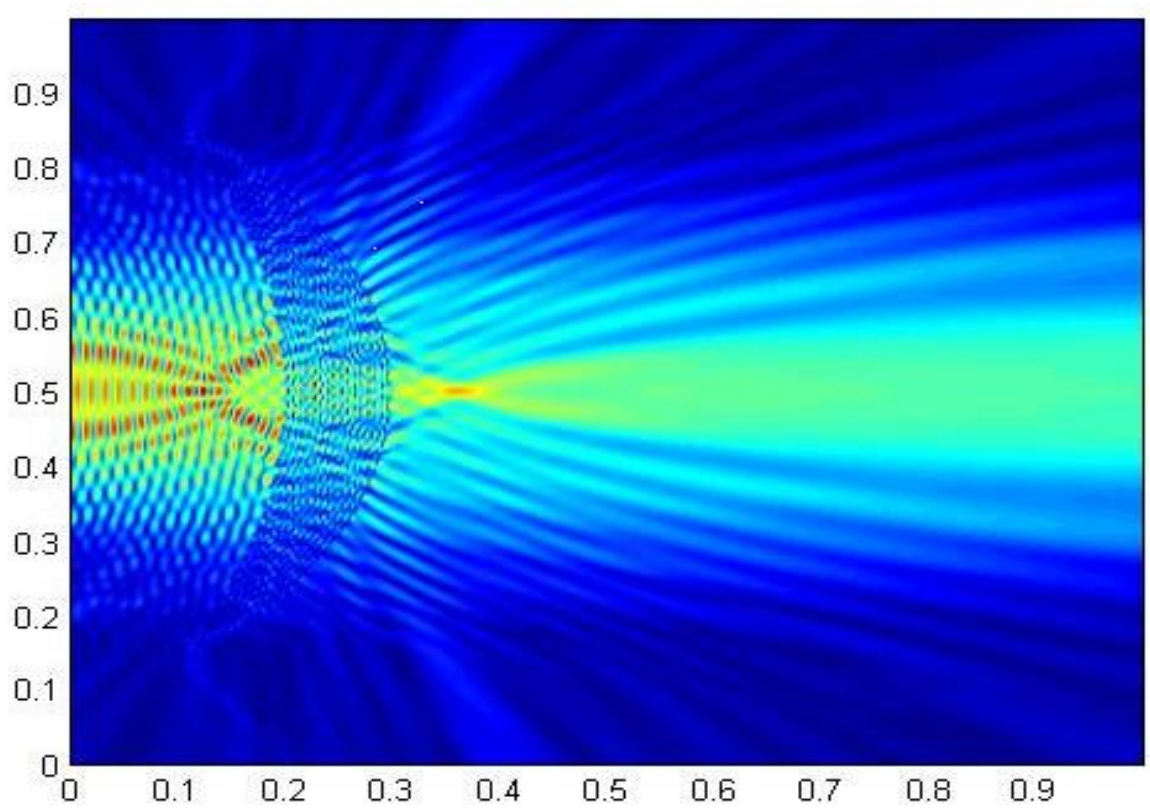

b)

Figura 5.6: Módulo del campo eléctrico en transmisión de a) antena lente hiperbólica y b) antena lente elíptica. Los ejes indican las dimensiones del cuadrado unidad.

La Figura 5.7 muestra el diagrama de radiación del campo lejano para la antena lente hiperbólica (a) y para la antena lente elíptica (b). 


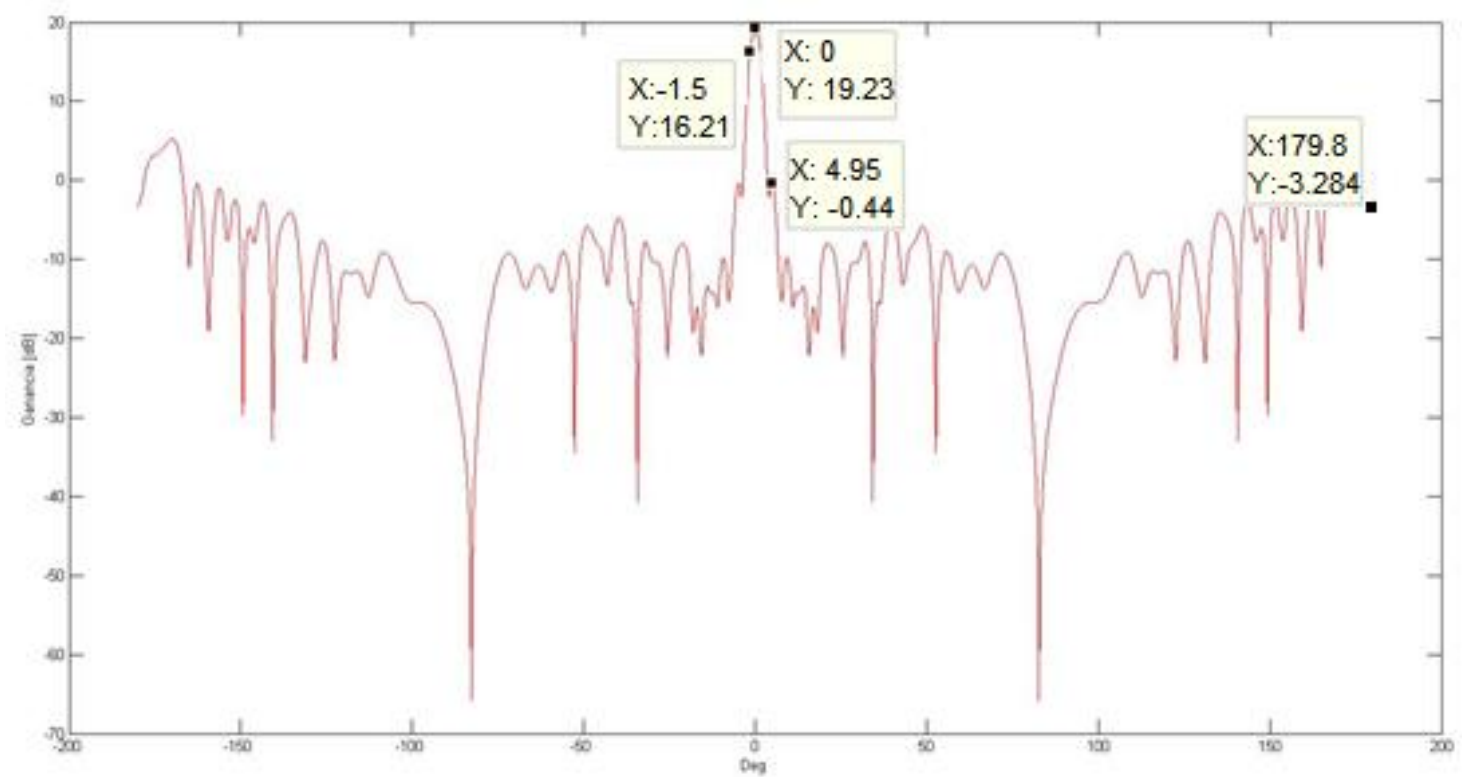

a)

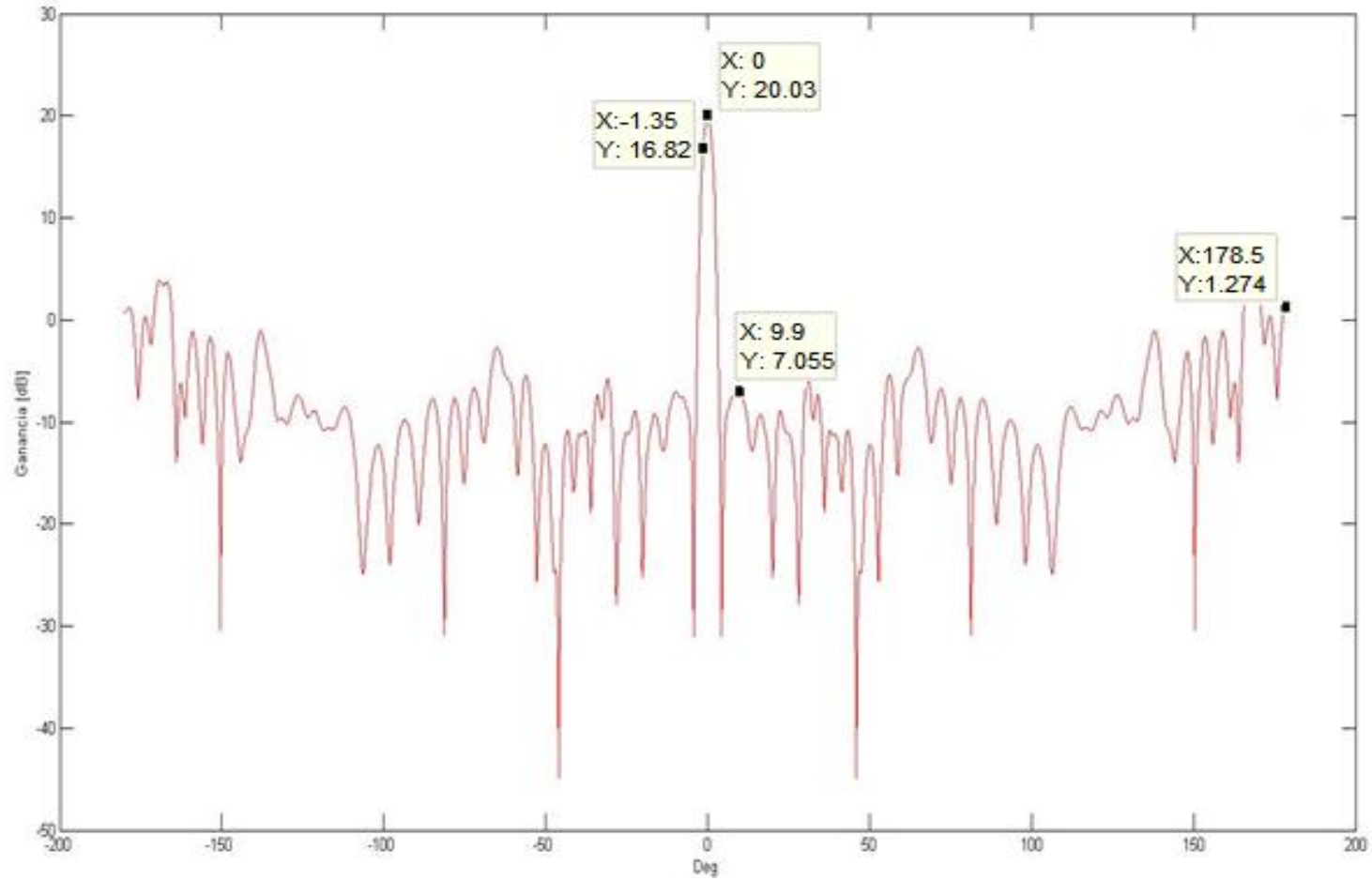

b)

Figura 5.7: Diagrama de radicación de a) antena lente hiperbólica y b) antena lente elíptica.

A partir de los resultados de las simulaciones, Figuras 5.7 a) y b) se calculan los parámetros característicos de la antena, ganancia $(\mathrm{G})$, ancho de haz $\left(\Delta \theta_{3 \mathrm{db}}\right)$, relación lóbulo principal a secundario (NLPS) y la relación frente-espalda (front to back), los cuales se resumen en la Tabla 5.3. 
Tabla 5.3: Características eléctricas de las antenas lente Hiperbólica y elíptica.

\begin{tabular}{|c|c|c|c|c|c|}
\hline $\begin{array}{l}\text { Antena } \\
\text { Lente }\end{array}$ & $\begin{array}{c}\text { Ganancia } \\
\qquad \begin{array}{c}G \\
{[d B]}\end{array}\end{array}$ & $\begin{array}{c}\text { Ancho de } \\
\text { haz } \\
\qquad \theta_{3 d b} \\
\left.I^{o}\right]\end{array}$ & $\begin{array}{c}N L P S \\
{[d B]}\end{array}$ & $\begin{array}{c}\begin{array}{c}\text { front to } \\
\text { back }\end{array} \\
\text { [dB] }\end{array}$ & $\begin{array}{c}\text { Tiempo } \\
\text { computacional } \\
{[\mathrm{s}]}\end{array}$ \\
\hline Hiperbólica & 19,23 & $3^{\circ}$ & 19,67 & 22,51 & 208 \\
\hline Elíptica & 20,03 & $2,7^{\circ}$ & 27,08 & 18,76 & 211 \\
\hline
\end{tabular}

Tal como se observa en la Tabla 5.3 la ganancia y el ancho de haz de las antenas lente hiperbólica y elíptica son similares.

Para el parámetro del lóbulo principal a secundario (NLPS), la lente elíptica presenta mejores características. Por otro lado, teniendo en cuenta la relación frenteespalda (Front to Back) dicha lente presenta importantes desventajas frente a la hiperbólica. Esto se debe a que la superficie de incidencia en la lente elíptica es esférica mientras que en la hiperbólica es plana. Por lo tanto, la energía reflejada por el perfil esférico tiende a concentrarse en el eje longitudinal de la lente mientras en el caso de la lente hiperbólica la energía reflejada se dispersa más (Figura 5.6 a y b).

\subsubsection{Análisis de fase a la salida de una antena lente}

También es posible analizar la fase a la salida de una lente. Para obtener la mayor eficiencia es deseable que la fase a la salida de la lente sea lo más constante posible.

La Figura 5.8 muestra el módulo del campo dentro y fuera de una lente Hiperbólica en transmisión. 


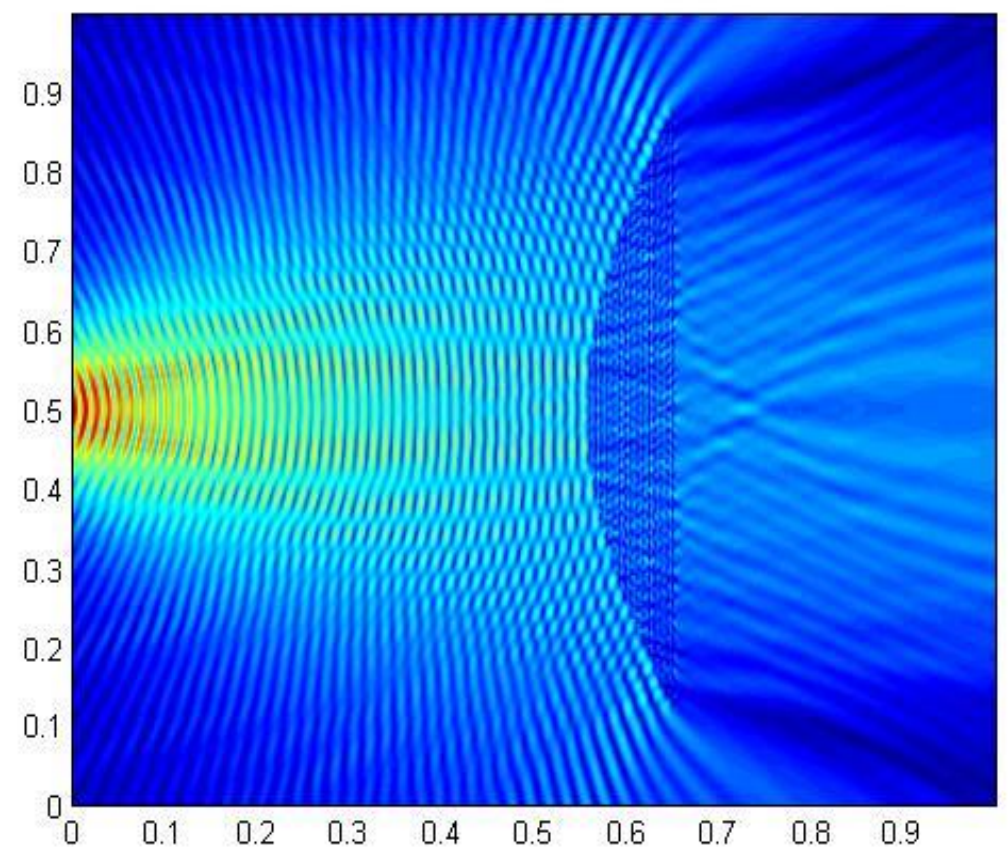

a)

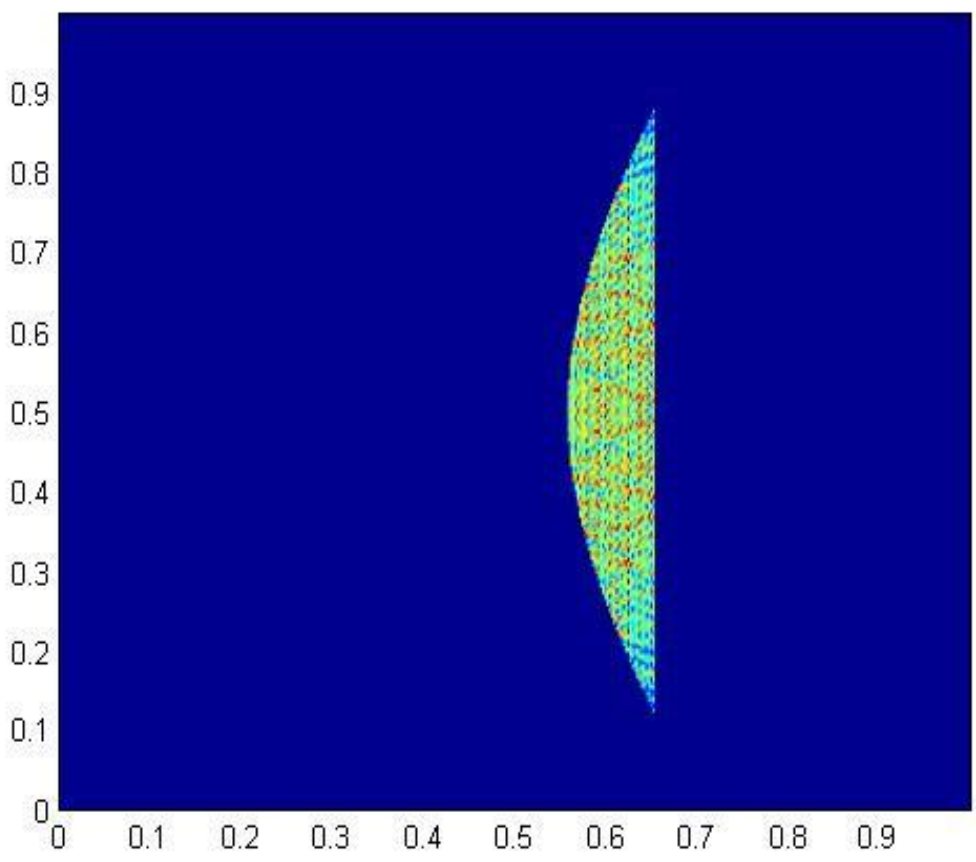

b)

Figura 5.8: Módulo del campo eléctrico cercano de una antena lente perfil hiperbólico a) dentro y fuera de la lente y b) dentro de la lente. Los ejes indican las dimensiones del cuadrado unidad. 
El espacio de simulación (cuadrado unidad) tiene dimensiones de $40 \lambda \times 40 \lambda$ ( $\lambda=1 / 40=0,025$ del cuadrado unidad). La fuente se encuentra fuera del cuadrado unidad (izquierda de la figura). Para obtener una buena resolución se simuló con $\mathrm{N}=400$ puntos (10 puntos por longitud de onda).

El diámetro de la lente es de $30 \mathrm{~cm}(30 \lambda)$ y la distancia focal es de $30 \mathrm{~cm}$. Con lo cual la relación $\mathrm{f} / \mathrm{D}=1$.

La Figura 5.9 muestra la fase del campo dentro y fuera de la lente. Se puede observar como la fuente genera ondas esféricas y al pasar por la lente la fase es cuasi plana. Para observar mejor la fase a la salida de la lente se puede graficar el perfil de la fase en un segmento vertical (línea color rojo) paralelo a la lente.
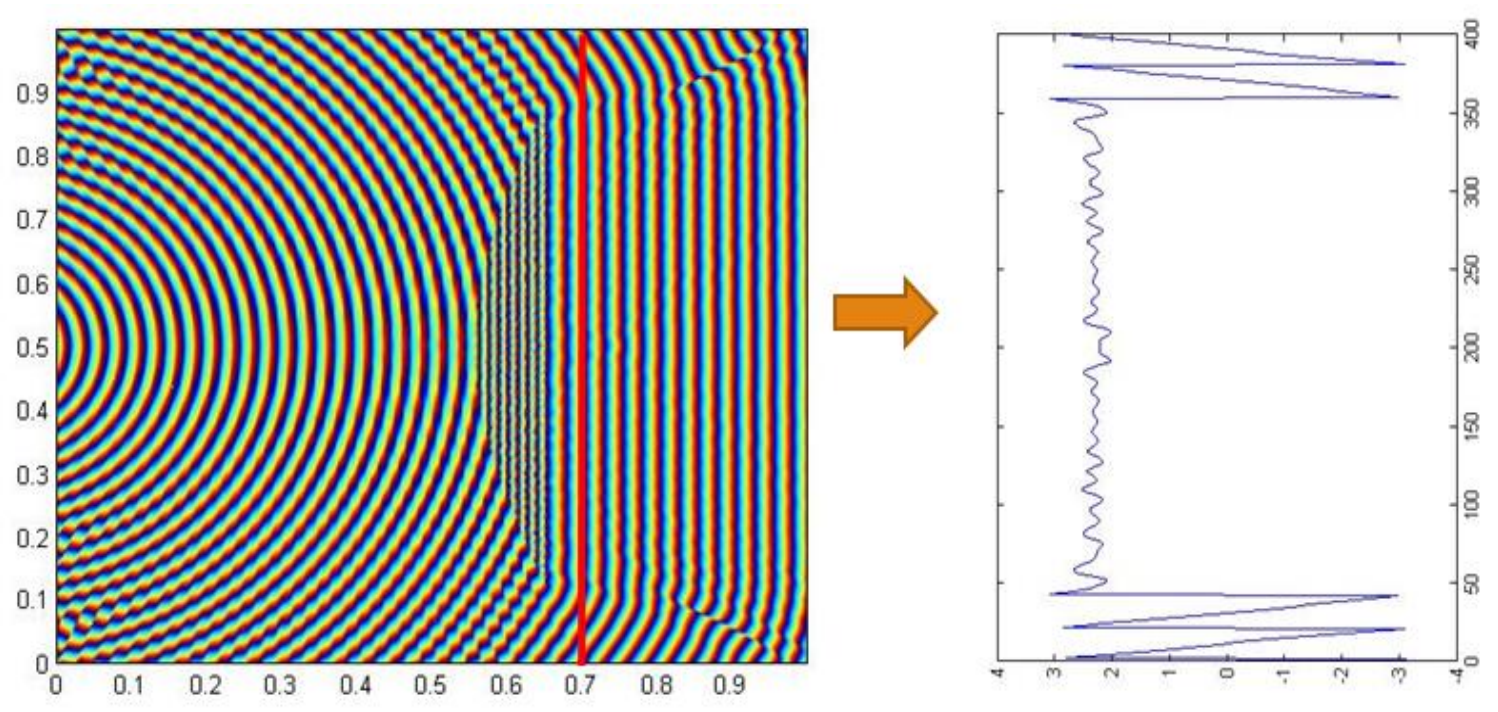

Figura 5.9: Fase del campo eléctrico dentro y fuera de la lente de perfil hiperbólico y fase del campo en la apertura. Los ejes indican las dimensiones del cuadrado unidad.

\subsubsection{Lente Escalonada de Fresnel}

Teniendo en cuenta la lente de Fresnel vista en 4.2.1.2 d) se diseñó y simuló una lente dieléctrica mediante el código de simulación [68] con una frecuencia central de diseño $\mathrm{f}_{0}=30 \mathrm{GHz}\left(\lambda_{0}=1 \mathrm{~cm}\right)$.

Para el diseño de la lente de Fresnel se toma como lente base una lente hiperbólica homogénea cuyas características físicas se detallan en la Tabla 5.4 [72]. 
Tabla 5.4: Características de la lente hiperbólica como base de la lente escalonada de Fresnel.

\begin{tabular}{|c|c|c|}
\hline $\begin{array}{c}\text { Diámetro } \\
{[\mathbf{c m}]}\end{array}$ & $\begin{array}{c}\text { Distancia } \\
\text { focal } \\
{[\mathbf{c m}]}\end{array}$ & $\begin{array}{c}\text { Permitividad } \\
\text { eléctrica } \\
{[\mathbf{\varepsilon r}]}\end{array}$ \\
\hline 30 & 28 & 4 \\
\hline
\end{tabular}

Se diseña una lente de $\mathrm{N}=2$ zonas y se extrae, a partir de la lente hiperbólica un cilindro de espesor $\mathrm{t}=1 \mathrm{~cm}$ de acuerdo a la ecuación 4.15 vista en el Capítulo 4 .

Con el objetivo de determinar la posición del foco de la lente, según se indica en la referencia [69], se irradia la lente con un frente onda plana de frecuencia $\mathrm{f}_{0}=30 \mathrm{GHz}$. El método consiste en determinar el campo máximo de concentración de campo eléctrico por detrás de la lente, obteniéndose la posición del foco de la misma y lograr un correcto posicionamiento del alimentador principal tal como se vio en 5.3.

El diagrama que se utiliza para simular la radiación del alimentador principal responde a la siguiente ecuación:

$$
S(\varphi)=(1+\cos (\varphi)) / 2)^{q}
$$

Según el criterio general de antenas de apertura, visto en el Capítulo 3, la máxima eficiencia de la lente se logra cuando la atenuación en bordes de la lente resulta de aproximadamente $-10 \mathrm{~dB}$ [61]. Para dicha condición y considerando la lente diseñada, se requiere un valor para la constante q de la ecuación (5.2) de 45.

El ancho de banda relativo teórico, según la ecuación (4.18), resulta 25\%, es decir: $\mathrm{Bw}=30+/-3,75 \mathrm{GHz}$.

La ganancia teórica de la antena-lente diseñada, para una eficiencia $\eta_{\mathrm{a}}$ del $50 \%$ y utilizando la ecuación (3.23), es de 19,75 dB.

Utilizando el software de simulación full-wave, se muestran en la Figura 5.10 los resultados obtenidos del módulo de campo eléctrico para la antena-lente de Fresnel diseñada. En dicha figura se puede observar en escala de intensidad de color el módulo del campo total dentro y fuera de la lente. El color rojo corresponde a las mayores intensidades de campo y el azul a las menores. 


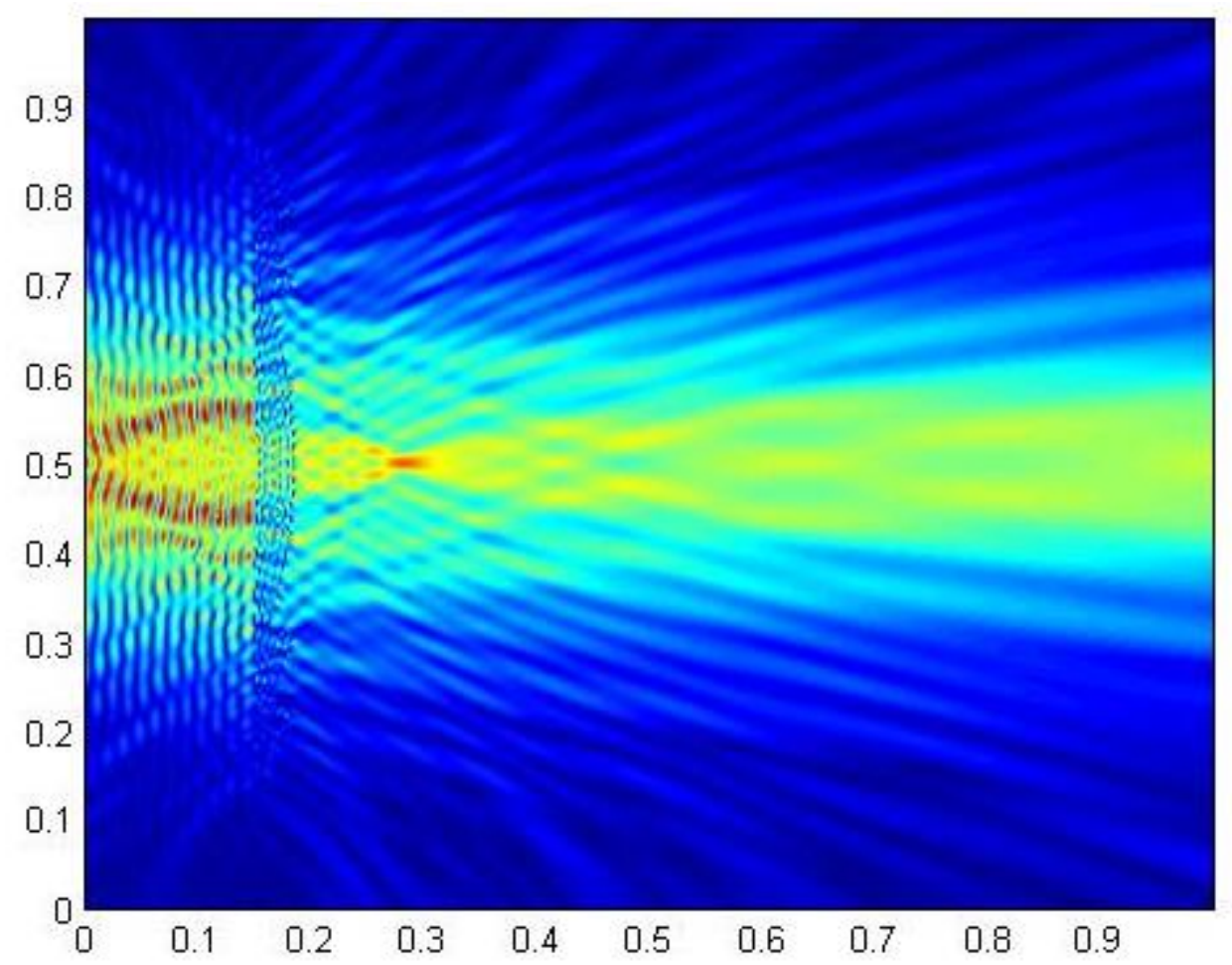

Figura 5.10: Módulo del campo eléctrico cercano de la lente de zonas de Fresnel. Los ejes indican las dimensiones del cuadrado unidad.

La Figura 5.11 muestra la variación de la ganancia de la lente en función de la frecuencia, presentando su mayor valor en la frecuencia central de diseño (30 GHz).

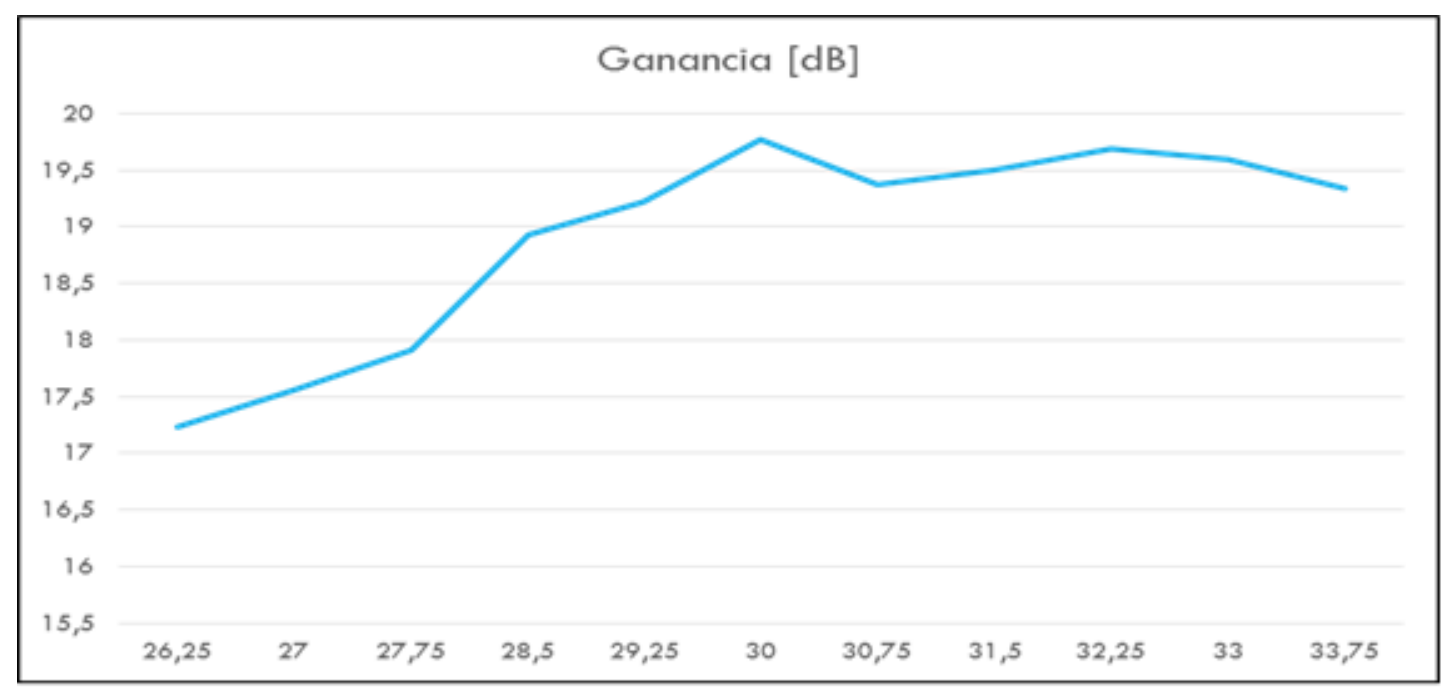

Frecuencia [Ghz]

Figura 5.11: Ganancia en función de la frecuencia para la antena lente de zonas de Fresnel. 


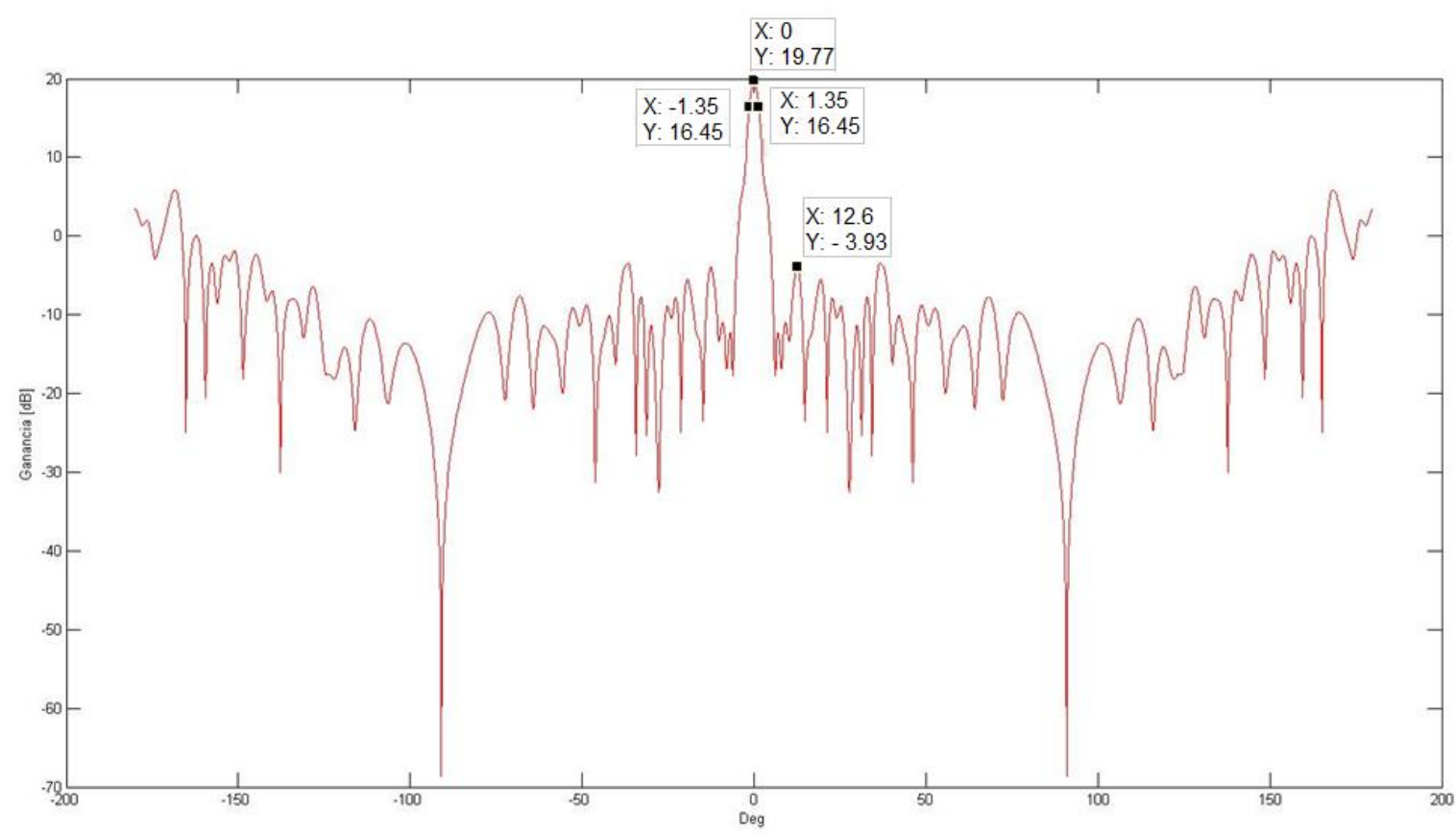

Figura 5.12: Diagrama de radiación de la antena lente de zonas de Fresnel.

Del diagrama de radiación obtenido para cada una de las frecuencias se pueden obtener la Ganancia $(\mathrm{G})$, el ancho del haz $\left(\Delta \theta_{-3} \mathrm{~dB}\right)$ y la relación lóbulo principal a secundario (NLPS), en función de la frecuencia.

El diagrama de radiación de la antena lente de Fresnel para $\mathrm{f}_{0}=30 \mathrm{GHz}$ se muestra en la Figura 5.12.

En la Tabla 5.5 se muestran las características de la antena lente de Fresnel dieléctrica, para la frecuencia central de $30 \mathrm{GHz}$ y en los extremos de un ancho de banda de 7,5 GHz, obtenidas de la simulación con el método full-wave.

La ganancia de la antena lente para la frecuencia central obtenida a partir de las simulaciones resulta de 19,77 dB; mientras que el valor teórico obtenido con la ecuación (6) es $19,75 \mathrm{~dB}$. 
Tabla 5.5: Características eléctricas de la antena lente de zonas de Fresnel para 3 frecuencias.

\begin{tabular}{|c|c|c|c|c|c|}
\hline $\mathbf{f}$ & $\boldsymbol{\lambda}$ & $\begin{array}{c}\text { Ganancia } \\
{[\mathbf{d B}]}\end{array}$ & $\begin{array}{c}\boldsymbol{\Delta} \boldsymbol{\theta}_{\mathbf{3}} \mathbf{d B} \\
{\left[^{\mathbf{}}\right]}\end{array}$ & $\begin{array}{c}\text { NLPS } \\
{[\mathbf{d B H}]}\end{array}$ & $\begin{array}{c}\text { Tiempo } \\
\text { computacional } \\
{[\mathbf{G}]}\end{array}$ \\
\hline 26,25 & 1,14 & 17,23 & 4,8 & 19 & 205 \\
\hline 30,00 & 1,00 & 19,77 & 2,5 & 22 & 221 \\
\hline 33,75 & 0,89 & 19,34 & 2,5 & 18 & 252 \\
\hline
\end{tabular}

Se observa que el método de simulación full-wave utilizado permite el correcto diseño y análisis de una antena-lente de Fresnel dieléctrica, para su utilización en las bandas de frecuencias de microondas.

Resulta interesante el comportamiento asimétrico de los parámetros que caracterizan a la antena alrededor de la frecuencia central siendo los cambios más sensibles para las frecuencias inferiores que para las superiores (Figura 5.11 y Tabla 5.5).

\subsubsection{Lente de Luneberg}

Mediante el código de simulación full-wave también es posible simular la lente de Luneberg tal como se describe en el inciso 4.2.1.2 del Capítulo 4. En este caso se simula una lente de radio $\mathrm{R}_{0}=27 \lambda_{0}$.

En la Figura 5.13 se observa a) la lente simulada para un espacio unidad de $60 \lambda_{0}$ x $60 \lambda_{0} \mathrm{y} \mathrm{b}$ ) un gráfico ampliado de la zona donde se encuentra ubicada la fuente (lado derecho de la lente). En ambas figuras es posible observar cómo la fuente genera ondas esféricas y al pasar por la lente se obtiene una fase constante (lado derecho de la lente).

El tiempo de simulación fue de 449 segundos. 


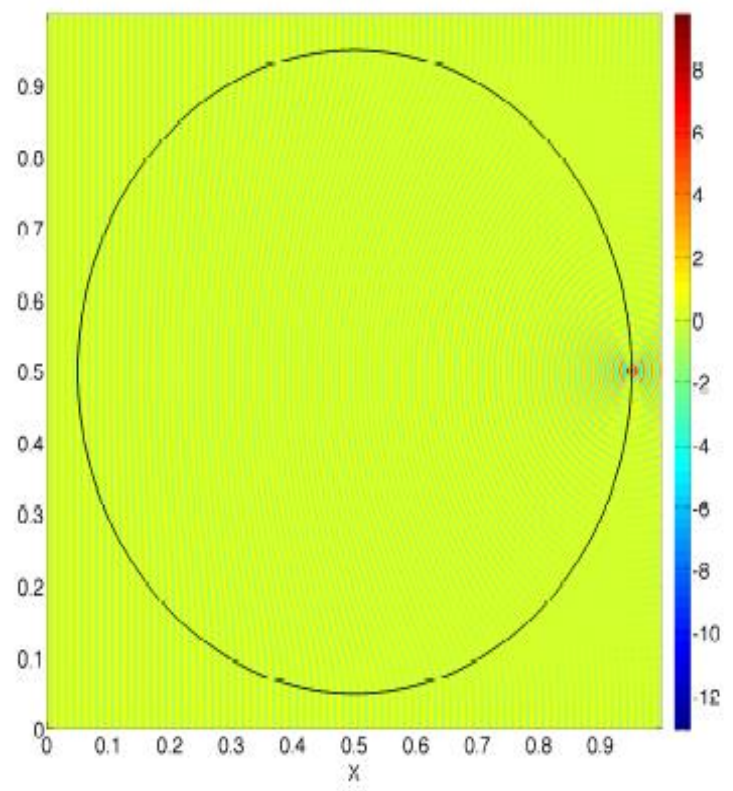

a)

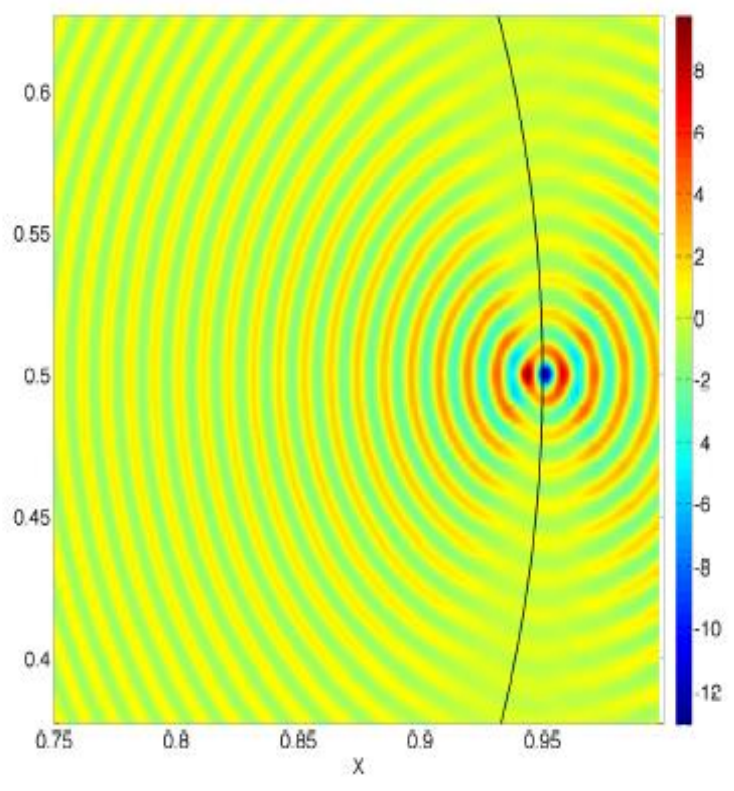

b)

Figura 5.13: Lente de Luneberg.

\subsection{Vórtices ópticos en antenas lentes en microondas}

\subsubsection{Vórtices ópticos}

Una onda electromagnética puede presentar singularidades de fase a lo largo de líneas continuas donde la amplitud de la onda se desvanece. Existen tres interpretaciones para estos defectos: dislocaciones del frente de onda, ya que los patrones de las superficies de fase constante (frentes de onda) reflejan los de las dislocaciones en los arreglos de los átomos en los cristales; como vórtices, ya que la dirección del gradiente de fase (es decir, la dirección de la corriente de energía o del vector de Poynting) gira alrededor de la línea singular como fluido en un vórtice irrotacional; y como ceros, es decir, "hilos de oscuridad" [73-75]. Las dislocaciones de fase son también conocidas como tenedor del diablo (Devil's Fork).

Estas singularidades de fase, vórtices o dislocaciones ópticas, se asocian con los lugares del frente de onda en donde la intensidad del campo luminoso se anula y por tanto la fase no está definida. Alrededor de este sitio, es decir del vórtice, el frente de onda (de fase constante) presenta una superficie de hélice con salto de $\pm 2 \pi \mathrm{m}$ durante una vuelta; donde $\mathrm{m}$ es la carga topológica [76]. La configuración helicoidal del frente de onda queda expresada por la dependencia espacial de la fase del haz en la forma: 


$$
\Phi(\varphi, z)=k z+m \varphi
$$

donde $\mathrm{z}$ es el eje de propagación, $\varphi$ es el ángulo azimutal en la sección transversal del haz, m es un entero denominado carga topológica de un VO y k es el número de onda.

En la Figura 5.14 se observa el efecto de las dislocaciones en un diagrama de fase para $\mathrm{m}=1 \mathrm{y} \mathrm{m}=5$ respectivamente.

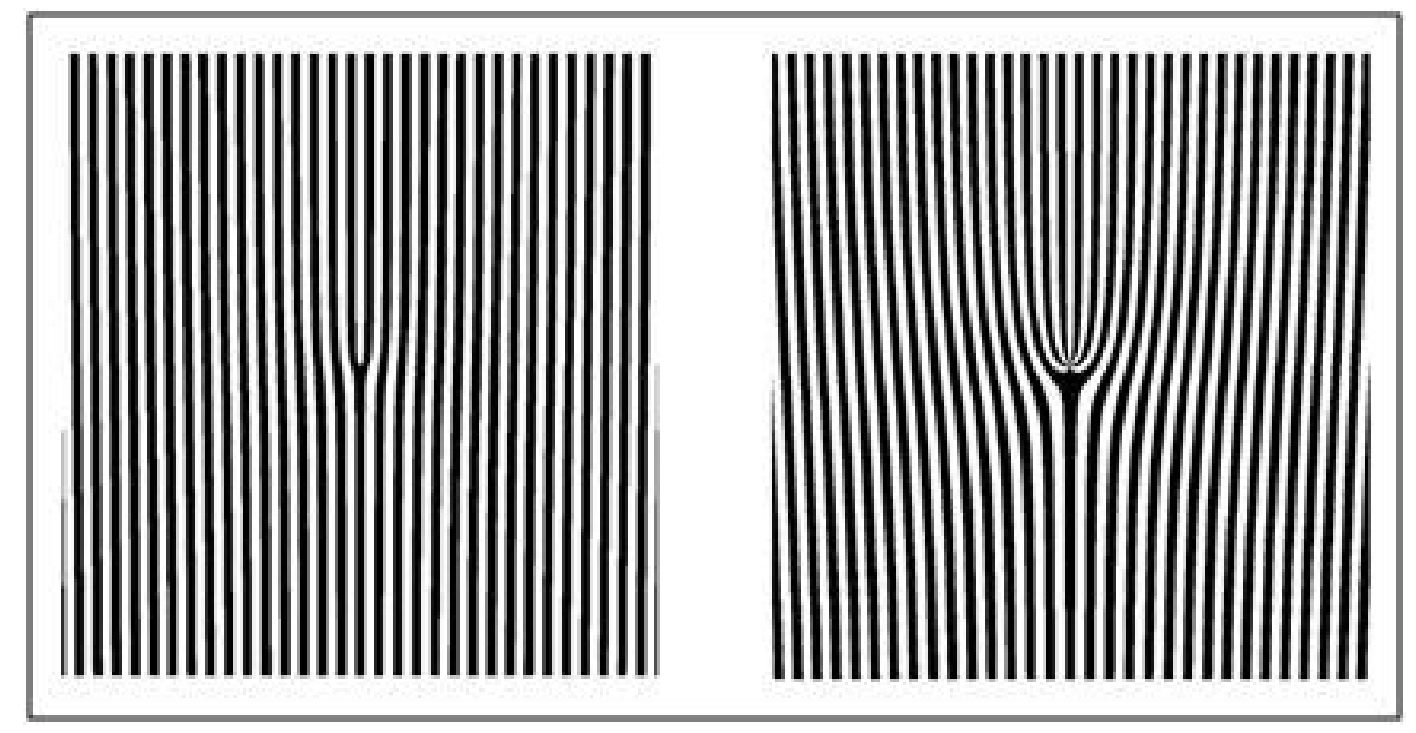

Figura 5.14: Vórtices ópticos de primer orden para una carga topológica de $m=1$ y $\mathrm{m}$ $=5$ respectivamente.

El patrón espiral del flujo de energía justifica el término "vórtice óptico" y se utiliza extensamente en ciencia óptica. Un vórtice óptico puede ser generado por la interacción de tres o más ondas planas [76].

Una onda plana puede ser descripta de la forma:

$$
U=U_{0} e^{i \vec{k} \vec{r}}
$$

donde $\mathrm{U}$ es la amplitud compleja del campo, $\mathrm{U}_{0}$ es la amplitud del campo, $\mathrm{k}$ es el vector de onda, que indica la dirección de propagación de la onda y $\mathrm{r}$ es el vector posición en el espacio.

En el caso de interferencia de dos ondas planas de igual amplitud $\mathrm{U}_{0}$, se obtiene el campo complejo dado por:

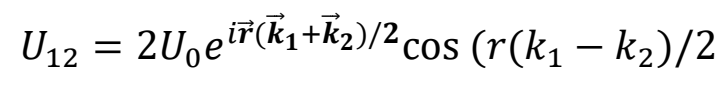


donde el término cosenoidal es el causante de la estructura periódica de franjas claras y oscuras, típicas de la interferencia entre dos ondas planas.

El campo total dadas tres ondas planas puede calcularse mediante la siguiente ecuación:

$$
U_{\text {total }}=\left(U_{12}+U_{23}+U_{13}\right) / 2
$$

Mientras que los vórtices ópticos producidos por rayos de luz son objeto de intensa actividad de investigación, habiéndose descubierto en ellos muchas propiedades interesantes, el fenómeno de vórtices en antenas lente en radiofrecuencias y microondas no ha recibido el mismo tratamiento.

A continuación, se analiza la manifestación de vórtices en antenas lente para microondas, resultados que podrían hacerse extensivos a ondas electromagnéticas de cualquier frecuencia.

\subsubsection{Simulaciones para evaluar vórtices ópticos en microondas}

Con el fin de evaluar la posible aparición de vórtices ópticos en antenas-lente se diseña y simula mediante el código 2D una lente dieléctrica de perfil hiperbólico y se modifican los bordes con el fin de ver dicho efecto sobre el frente de onda.

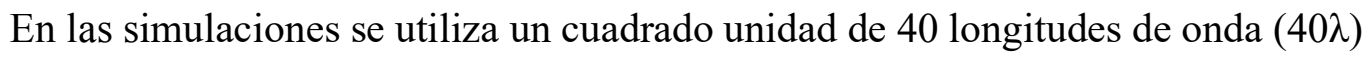
de lado, y $\mathrm{N}=400$.

La lente base analizada posee un diámetro de 30 longitudes de onda (30入) y su constante dieléctrica relativa es de un valor de cuatro $\left(\varepsilon_{\mathrm{r}}=4\right)$. Al estar diseñada la lente en función de la longitud de onda de la frecuencia del campo a irradiar, los resultados se hacen extensivos a cualquier frecuencia.

La fuente es colocada en el foco de la lente tal como se determinó en el inciso 5.3 , quedando fuera del cuadrado unidad definido para la simulación.

Se realizan simulaciones con la lente base y con la lente modificada en sus bordes. Ambas lentes poseen igual diámetro, perfil y material dieléctrico. La lente modificada se logra incrementando el espesor de la lente base, modificación que no altera la dirección del frente de la onda que atraviesa la lente. Las simulaciones no contemplan las pérdidas en el material dieléctrico, por lo que el cambio de espesor no debería modificar la ganancia de la lente. La alteración de los bordes de la lente es ensayada con la intención de ver cómo se afectan los diagramas de módulo y fase de los campos electromagnéticos de la antena-lente. 


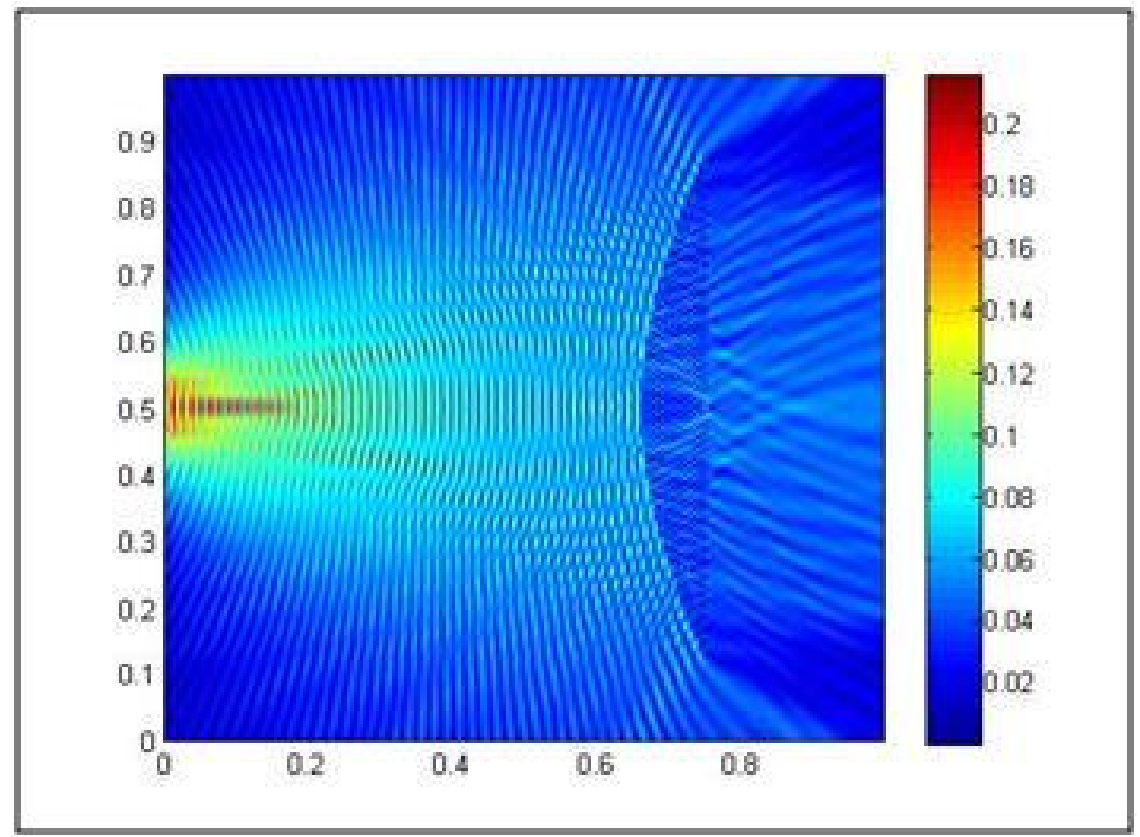

a)

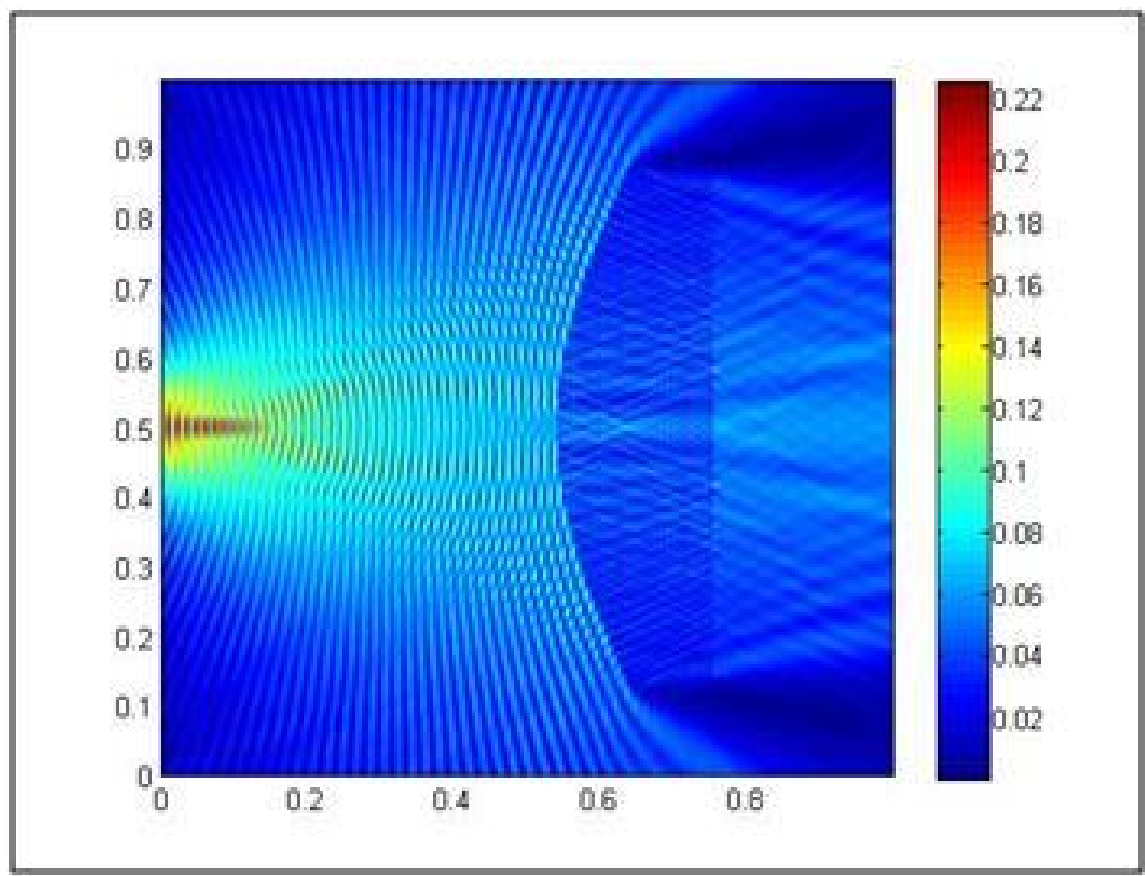

b)

Figura 5.15: Intensidad relativa del campo dentro y fuera de la lente en el espacio de simulación (cuadrado unidad) para la lente base (a) y la lente modificada (b).

En la Figura 5.15 a) y b) se muestran gráficos en los cuales el color es indicativo de la intensidad del campo electromagnético normalizado (adimensional) dentro del cuadrado unidad para las dos lentes simuladas. 
Se observa que la presencia de bordes planos (Figura 5.15 b) modifica la intensidad del campo respecto de la lente base fundamentalmente en la región cercana a los bordes donde una zona de color azul intenso evidencia la reducción del módulo hasta su posible anulación (Figura 5.15 a).

En la Figura 5.16 a) y b) se observa el diagrama de fase del campo en la lente base y en la lente modificada, respectivamente. Para esta última, en la zona de los bordes planos superior e inferior se observan dislocaciones en el diagrama de fase (Figura 5.16 b), coincidiendo esta región con aquella en la que el módulo prácticamente se anula (Figura 5.16 b).

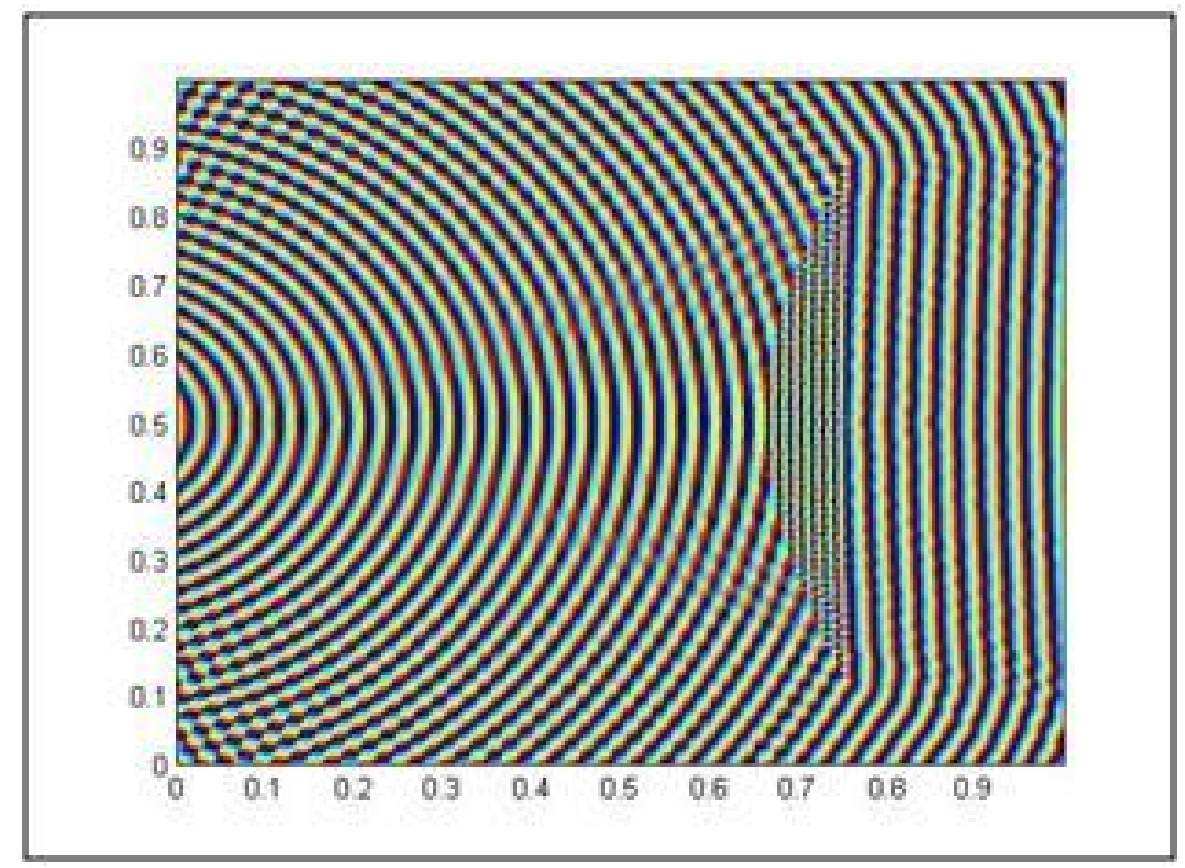

a) 


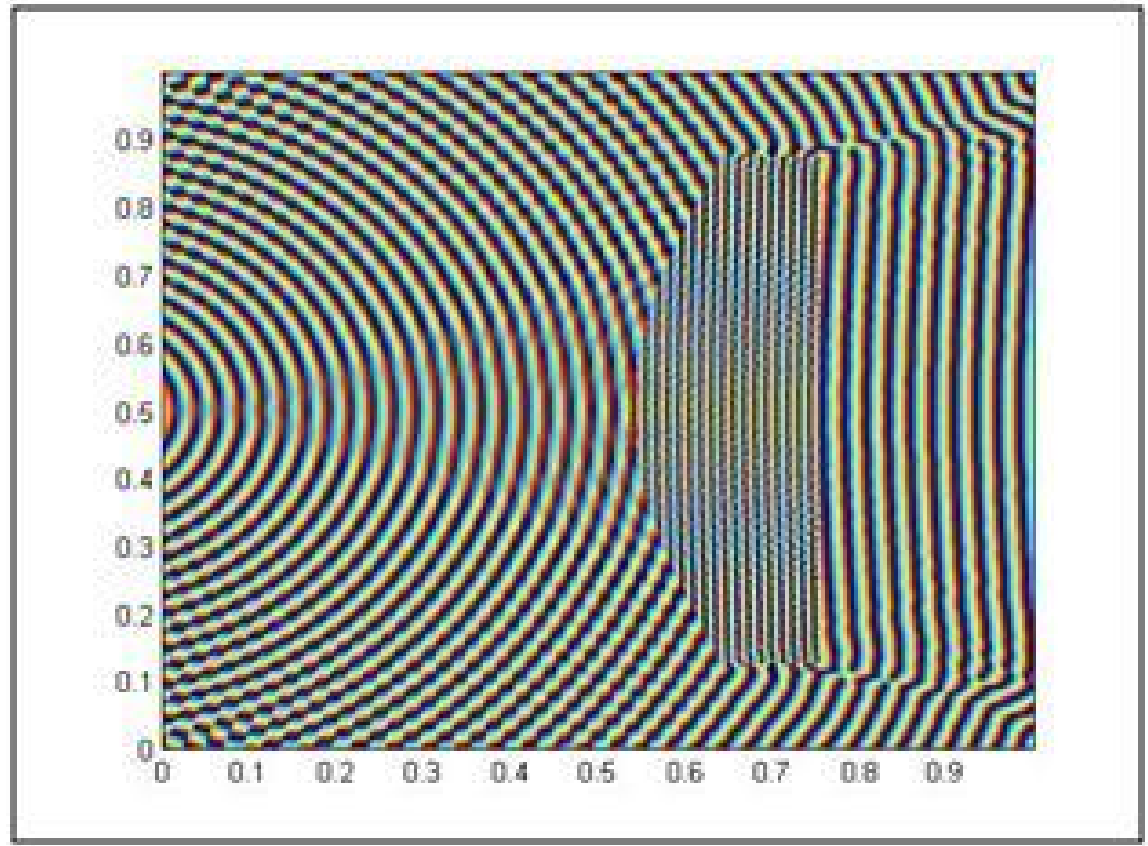

b)

Figura 5.16: Fase del campo dentro y fuera de la lente. a) Lente base y b) Lente modificada.

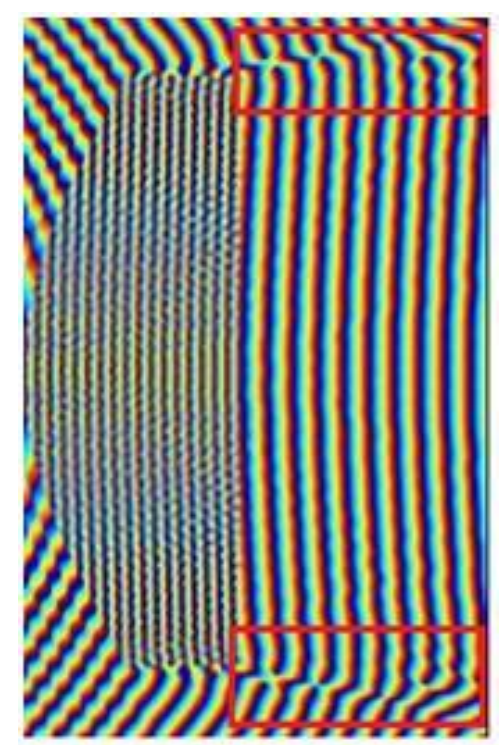

Figura 5.17: Ampliación de la Fig. 4b) del sector donde se ubican las dislocaciones.

La Figura 5.17 muestra una ampliación del sector en el diagrama de fase donde se ubican las dislocaciones.

La observación de estas dos condiciones en un mismo sitio, módulo de campo nulo y presencia de dislocaciones de fase, nos permite inferir la existencia de vórtices en 
dichas zonas. Tratándose del mismo fenómeno ampliamente estudiado en el espectro visible, del cual deriva la denominación vórtices ópticos $\mathrm{VO}$, ésta se conserva aún para las microondas.

El software utilizado genera adicionalmente el diagrama polar de radiación (campo lejano), volcado en la Figura 5.18 a) y b) para ambos casos estudiados. En color rojo se percibe el diagrama polar de campo lejano de la fuente aislada, mientras que en color azul se percibe el diagrama total del conjunto alimentador lente.

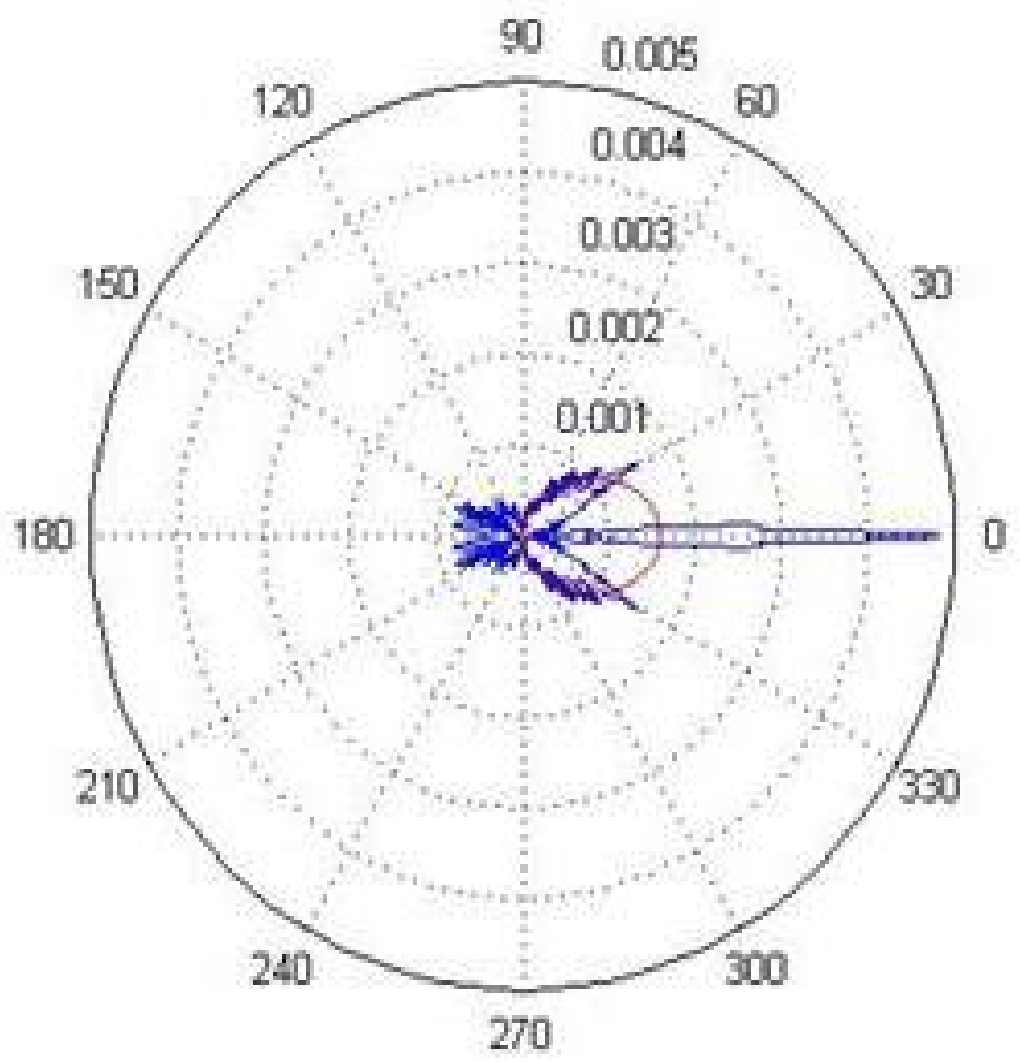

a) 


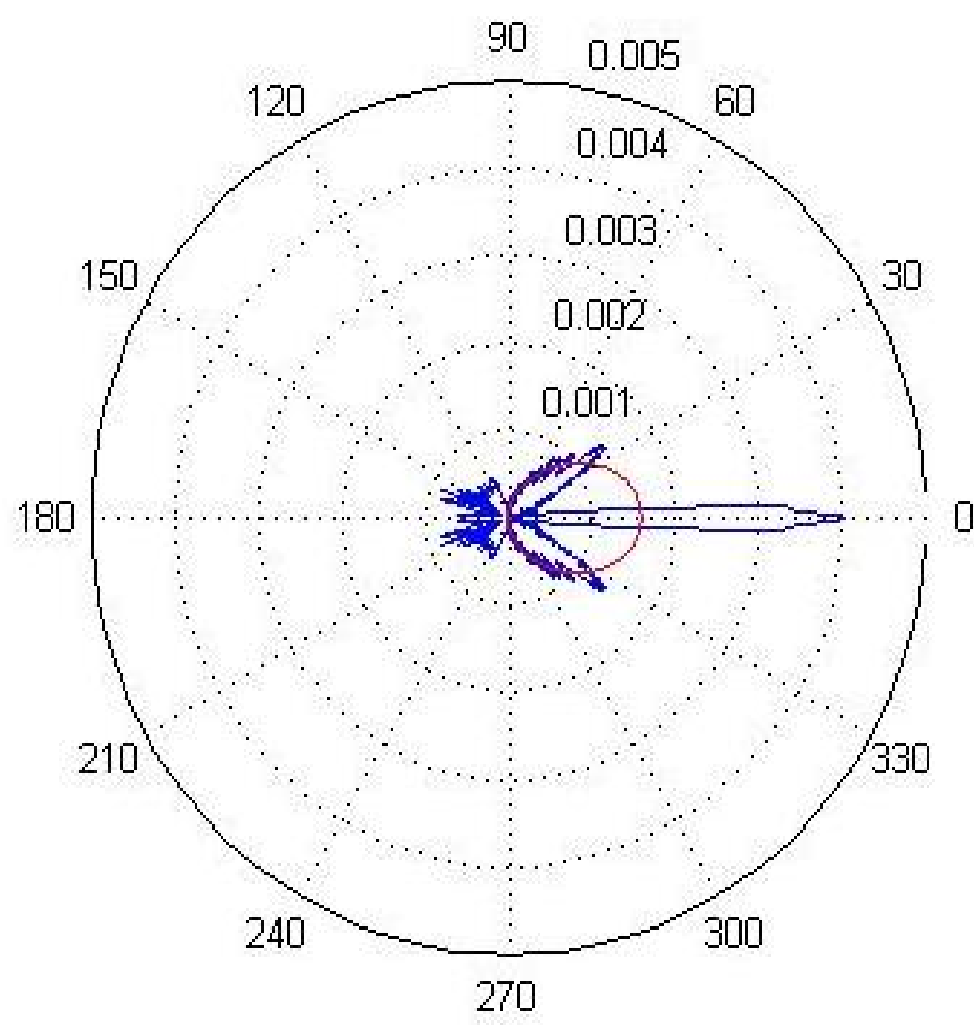

b)

Figura 5.18: Diagrama polar de a) la lente base y b) la lente modificada, es decir con bordes planos.

Es notoria la reducción de la ganancia, respecto de la lente base, manifiesta en el lóbulo principal del conjunto antena-lente cuando ésta presenta bordes planos (Figura $5.18 \mathrm{~b}$ ). Este efecto observado en la antena-lente modificada podría estar relacionado con la presencia manifiesta de los vórtices $[77,78]$.

\subsection{Capas adaptativas en lentes de microondas}

Como se vio en el Capítulo 4, el espesor de las lentes dieléctricas está directamente relacionado con su diámetro y la permitividad relativa del material del que está construida. En muchas aplicaciones, donde el tamaño y/o el peso de la lente es un factor limitante, puede reducirse el espesor de la lente si se utiliza un material de mayor constante dieléctrica. Esto trae aparejado un aumento de las pérdidas por reflexión del conjunto antena lente.

Las pérdidas por reflexión se pueden aproximar para la mayoría de las lentes, según la bibliografía, con la ecuación (3.31) vista en el Capítulo 3. 


$$
L=8,69 \frac{(n-1)^{2}}{(n+1)^{2}}(d b)
$$

donde $\mathrm{n}=\sqrt{ }\left(\varepsilon_{\mathrm{r}}\right)$ es el índice de refracción correspondiente al material.

Una alternativa para disminuir las pérdidas por reflexión es la aplicación de una capa adaptativa (matching layer) de cuarto de onda en la lente.

\subsubsection{Capas adaptativas en dieléctricos}

Si se tienen dos medios de diferentes características eléctricas tal como se muestra en la Figura 5.19, se puede determinar el coeficiente de reflexión para una onda incidente a partir de la impedancia intrínseca de los dos medios. Siendo la impedancia intrínseca:

$$
Z_{i}=\sqrt{\frac{\mu_{0}}{\varepsilon_{0}} \frac{\mu_{r_{i}}}{\varepsilon_{r_{i}}}}
$$

donde $\varepsilon_{0}$ la permitividad del vacío $(\mathrm{F} / \mathrm{m}), \varepsilon_{\mathrm{ri}}$ la permitividad relativa del medio i, $\mu_{0}$ la permeabilidad del vacío $(\mathrm{H} / \mathrm{m})$ y $\mu_{\text {ri }}$ la permeabilidad relativa del medio $i$.

El coeficiente de reflexión será:

$$
\Gamma=\frac{Z_{2}-Z_{1}}{Z_{2}+Z_{1}}
$$

donde $\Gamma$ es el coeficiente de reflexión, $Z_{1}$ la impedancia intrínseca del medio 1 y $\mathrm{Z}_{2}$ la impedancia intrínseca del medio 2 . La suma de las ondas incidente y reflejada en el medio 1 genera una onda parcialmente estacionaria. En el medio 2 (considerado como infinito) sólo habrá una onda progresiva. 


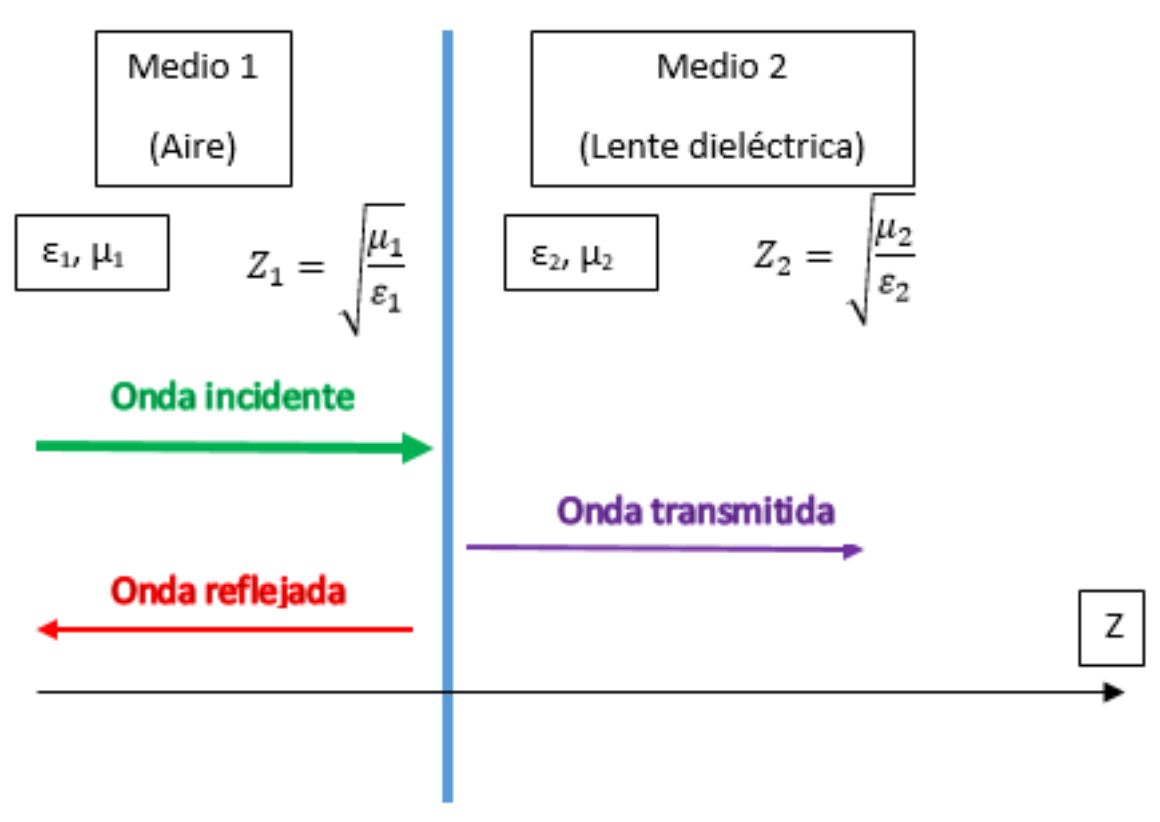

Figura 5.19: Características eléctricas de 2 medios y la ondas incidentes, reflejadas y transmitidas.

Se puede determinar la relación de onda estacionaria (ROE o VSWR) definida como la relación de la envolvente de campo máxima (eléctrica o magnética) a la envolvente de campo mínima, como:

$$
R O E=\frac{|E(z)|_{\text {max }}}{|E(z)|_{\text {min }}}
$$

La ROE está relacionada con el coeficiente de reflexión de acuerdo con la siguiente fórmula:

$$
R O E=\frac{1+|\Gamma|}{1-|\Gamma|}
$$

De las ecuaciones (5.8), (5.9) y (5.11) se puede deducir que, a mayor diferencia entre las permitividades dieléctricas de los dos medios, mayor será el coeficiente de reflexión y la relación de onda estacionaria.

Si se desea reducir el espesor de la lente, se deberá emplear un material dieléctrico con mayor permitividad lo que redundará en un mayor coeficiente de reflexión y mayor relación de onda estacionaria. Para subsanar este aumento, es posible adosar a la lente capas adaptativas de cuarto de onda.

El empleo de capas adaptativas es un recurso muy utilizado para disminuir el coeficiente de reflexión [79]. La adaptación se logra insertando entre los medios 
originales una capa de un dado material, que cumpla determinadas características (Figura 5.20).

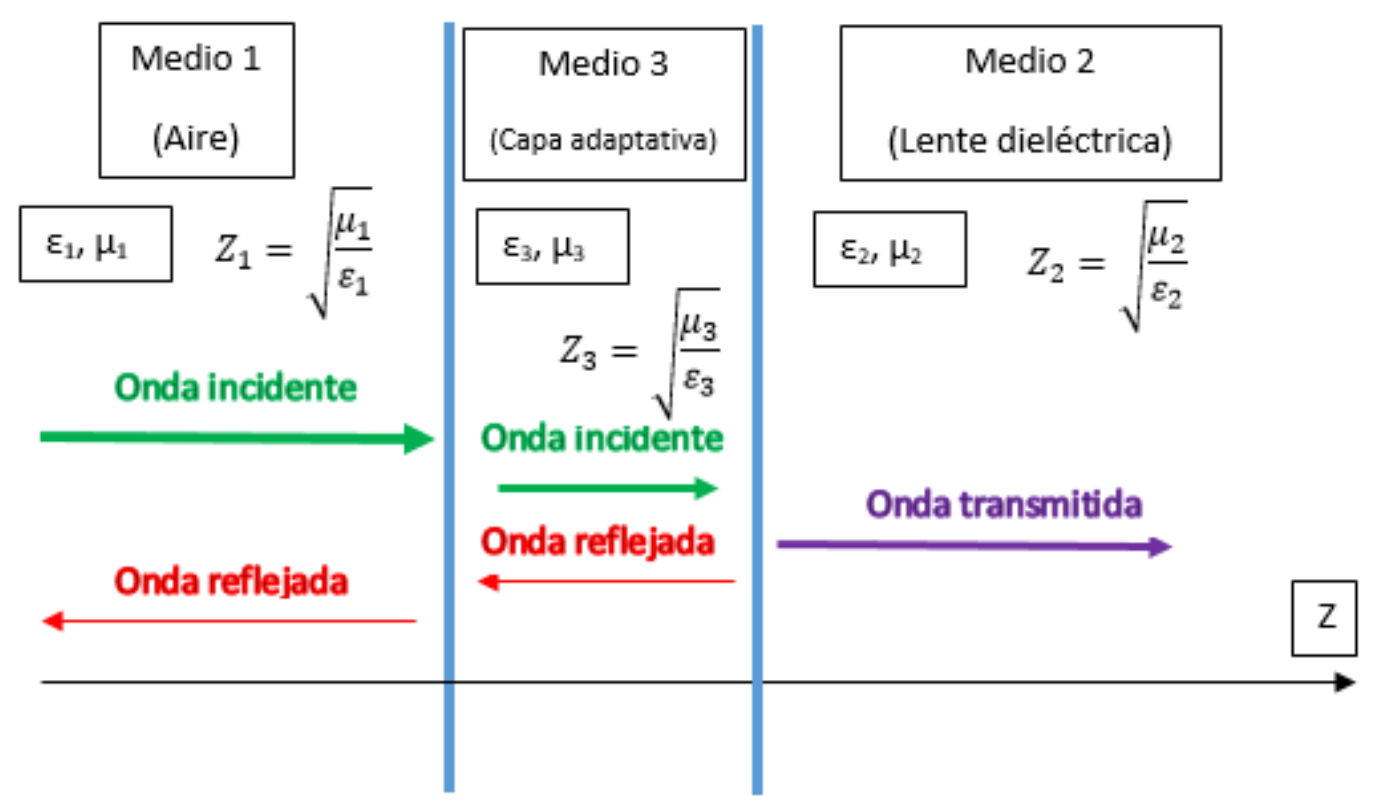

Figura 5.20: Características eléctricas de tres medios y la ondas incidentes, reflejadas y transmitidas.

La adaptación se logra cuando el material adaptador, medio 3 Figura 5.20, tiene un espesor igual a un cuarto de la longitud de onda en dicho medio y una impedancia intrínseca que cumple la siguiente ecuación [80]:

$$
\mathrm{Z}_{3}=\sqrt{\mathrm{Z}_{1} \cdot \mathrm{Z}_{2}}
$$

\subsubsection{Simulaciones de antena lente adaptada}

Con el fin de evaluar el efecto de la utilización de capas adaptativas en antenas lente dieléctricas se diseña una lente de perfil hiperbólico plano-convexo y se realizan simulaciones de una antena lente con y sin la capa adaptativa tal como se muestra en la Figura 5.21. 


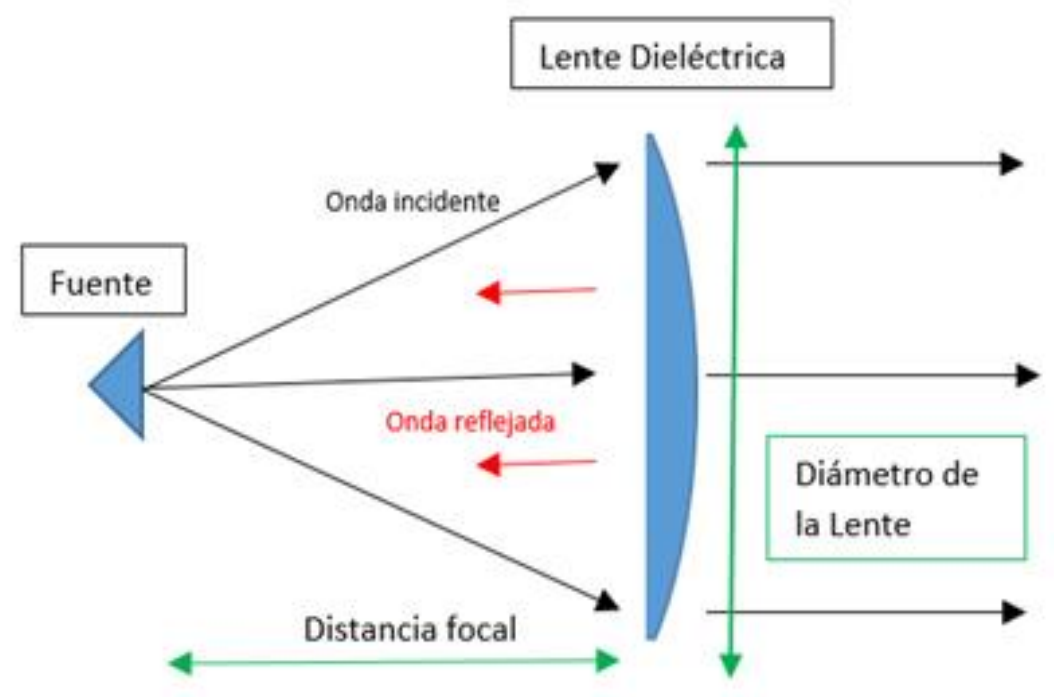

a)

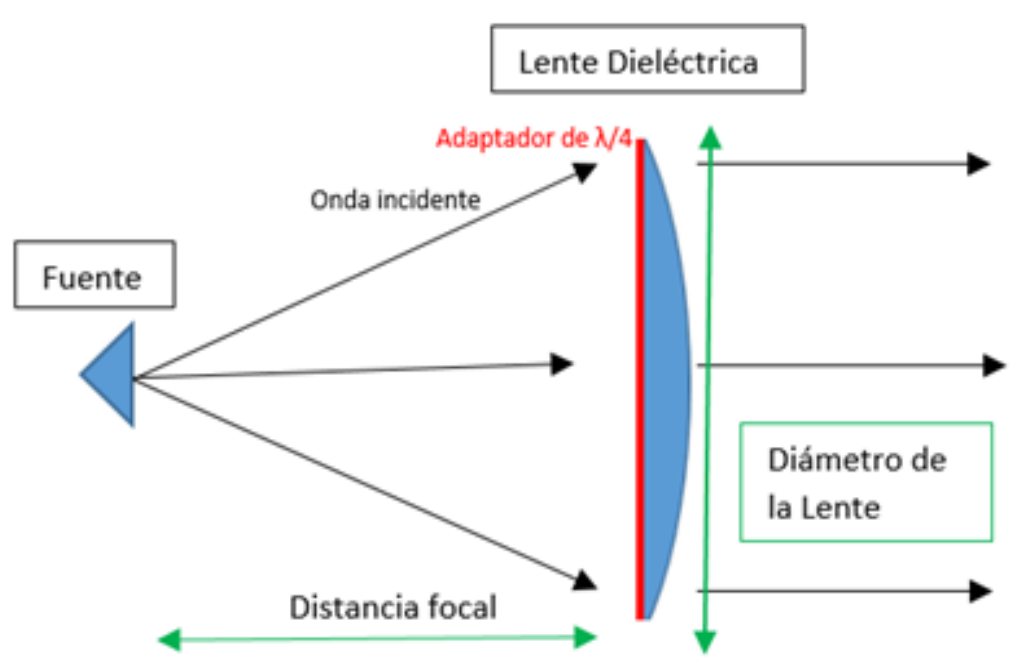

b) 


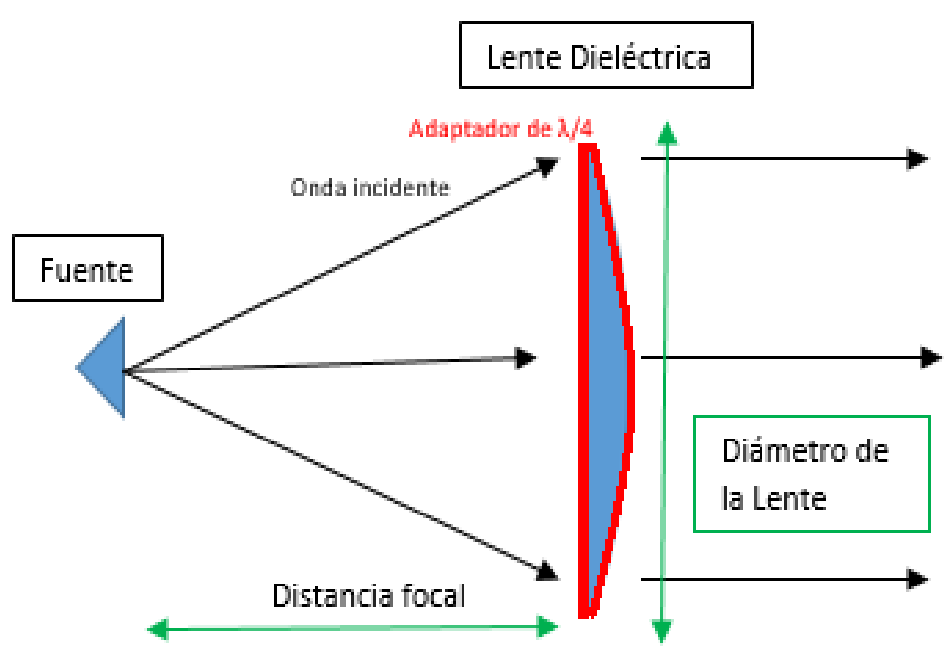

c)

Figura 5.21: Lente Hiperbólica a) sin adaptador, b) con adaptador y c) doble adaptador.

La lente base analizada posee un diámetro $\mathrm{D}=30 \mathrm{~cm}$ y un espesor $\mathrm{d}=4 \mathrm{~cm}$. Su constante dieléctrica relativa es de un valor de cuatro $\left(\varepsilon_{\mathrm{r}}=4\right)$. Se irradia la lente con una fuente a frecuencia de operación $\mathrm{f}=60 \mathrm{GHz}$, siendo la longitud de onda correspondiente $\lambda=5 \mathrm{~mm}$. A esta frecuencia el diámetro de la lente corresponde a $60 \lambda$ y el espesor es de $8 \lambda$. El cuadrado unidad tiene dimensiones de $80 \lambda$ x $80 \lambda$ y $\mathrm{N}=800$ puntos ( $\lambda \equiv 10$ puntos).

La fuente es colocada en el foco de la lente, quedando fuera del cuadrado unidad definido para la simulación.

Siendo $Z_{1}$ la impedancia intrínseca del aire, la impedancia intrínseca de la lente resulta $Z_{2}=Z_{1} / \sqrt{ } 4$. Por lo tanto, el medio de la capa adaptativa deberá tener una impedancia intrínseca, que según (5.8) será $Z_{3}=Z_{1} / \sqrt{ } 2$.

La longitud de onda para el medio de la capa adaptativa será $\lambda_{3}=\lambda / \sqrt{2}=3,53$, por lo que la capa adaptativa de cuarto de onda tendrá un espesor de $0,88 \mathrm{~mm}$, resultando despreciable su aporte en el peso total de la lente.

Se realizan simulaciones con la lente sin y con las capas adaptativas. Las simulaciones no contemplan las pérdidas de transmisión en el material dieléctrico.

La Figura 5.22 muestra un diagrama de la intensidad relativa del campo dentro y fuera de la lente en el espacio de simulación (cuadrado unidad) para la lente a) sin adaptador y b) doble adaptador. 


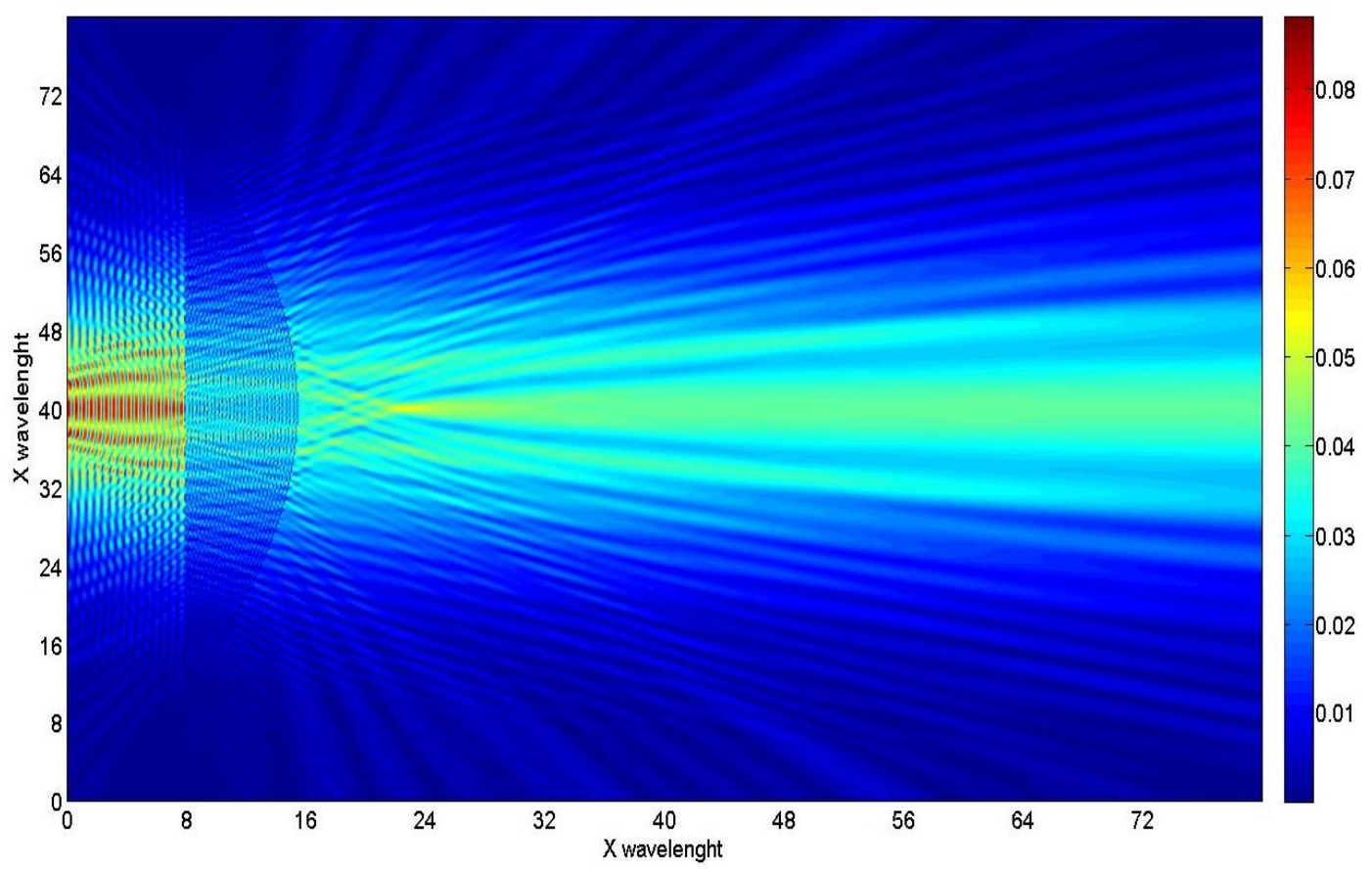

a)

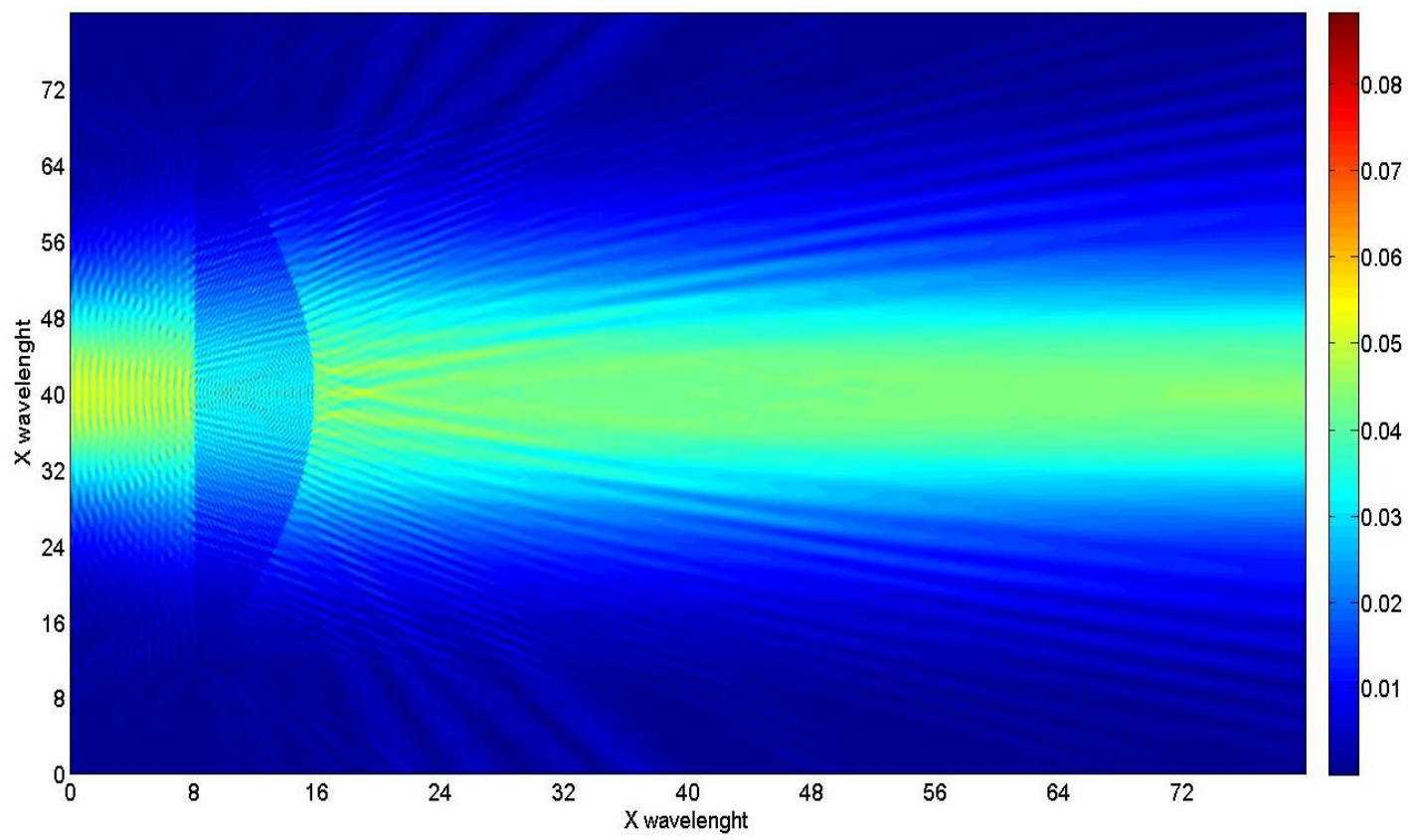

b)

Figura 5.22: Intensidad relativa del campo dentro y fuera de la lente hiperbólica a) sin adaptar y b) con doble adaptador.

Se observa a la izquierda de la Figura 5.22 en color rojo la onda estacionaria formada entre el alimentador y la lente debido a la suma de la onda incidente y la onda 
reflejada en la lente. La Figura 5.23 muestra el diagrama del módulo del campo en el eje de la lente para el caso de la lente a) sin adaptar (azul) y la lente con adaptador simple (rojo) y b) la lente sin adaptar (azul) y la lente con adaptador doble (fucsia).

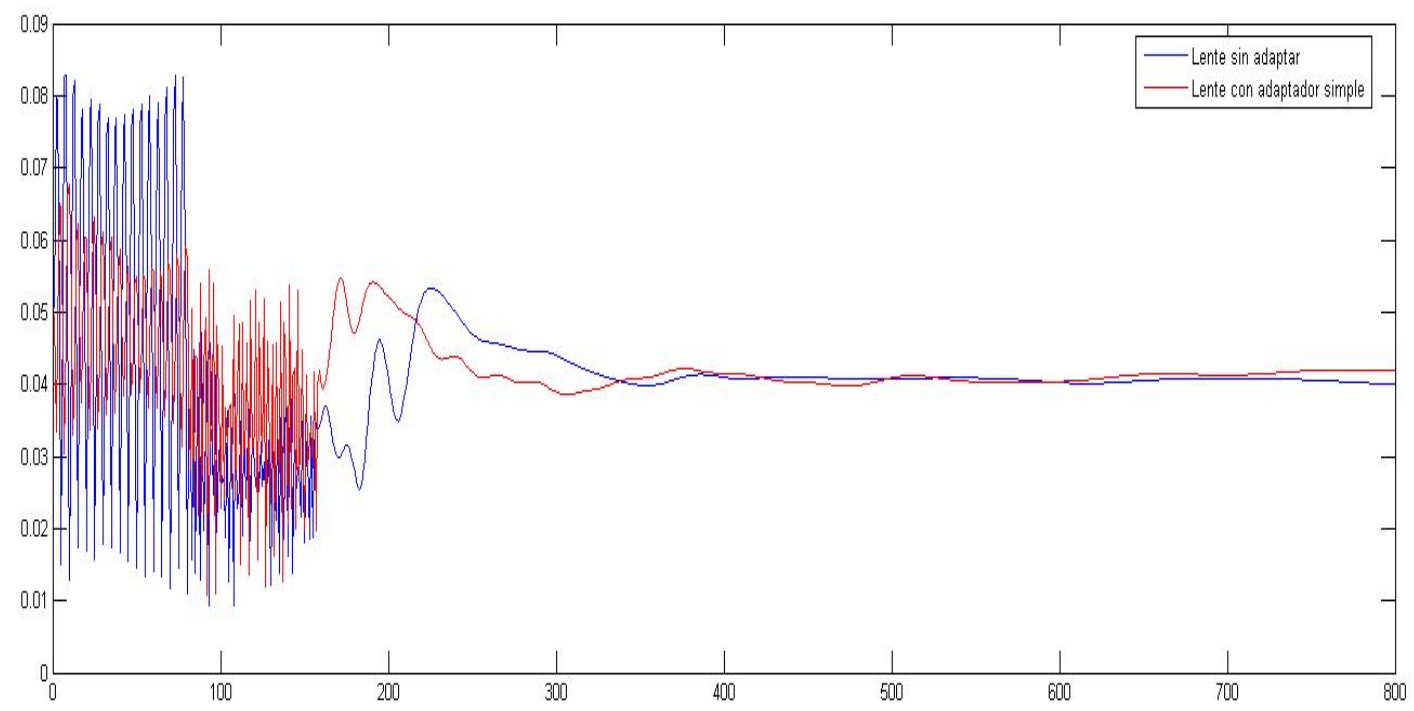

a)

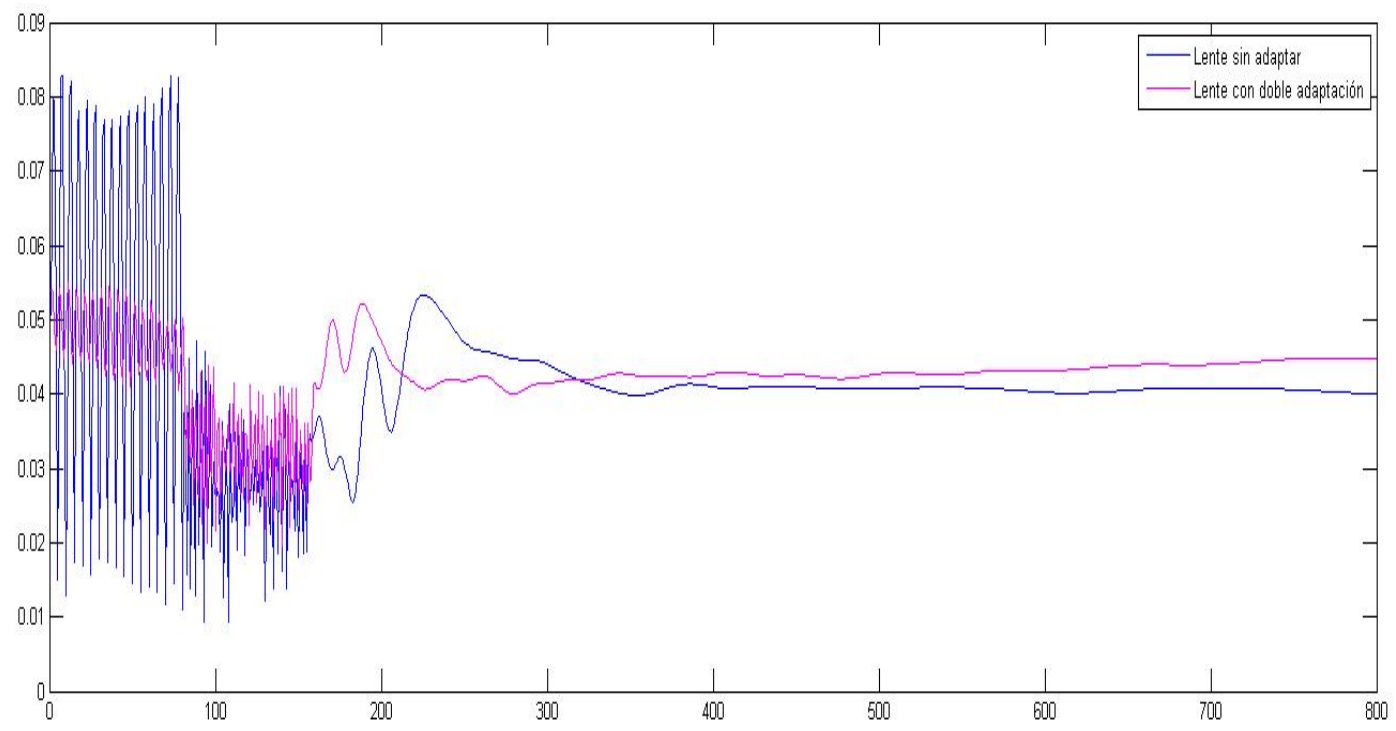

b)

Figura 5.23: Módulo del campo eléctrico en el eje de la lente hiperbólica a) sin adaptar y con adaptador simple y b) sin adaptar y con adaptador doble. 


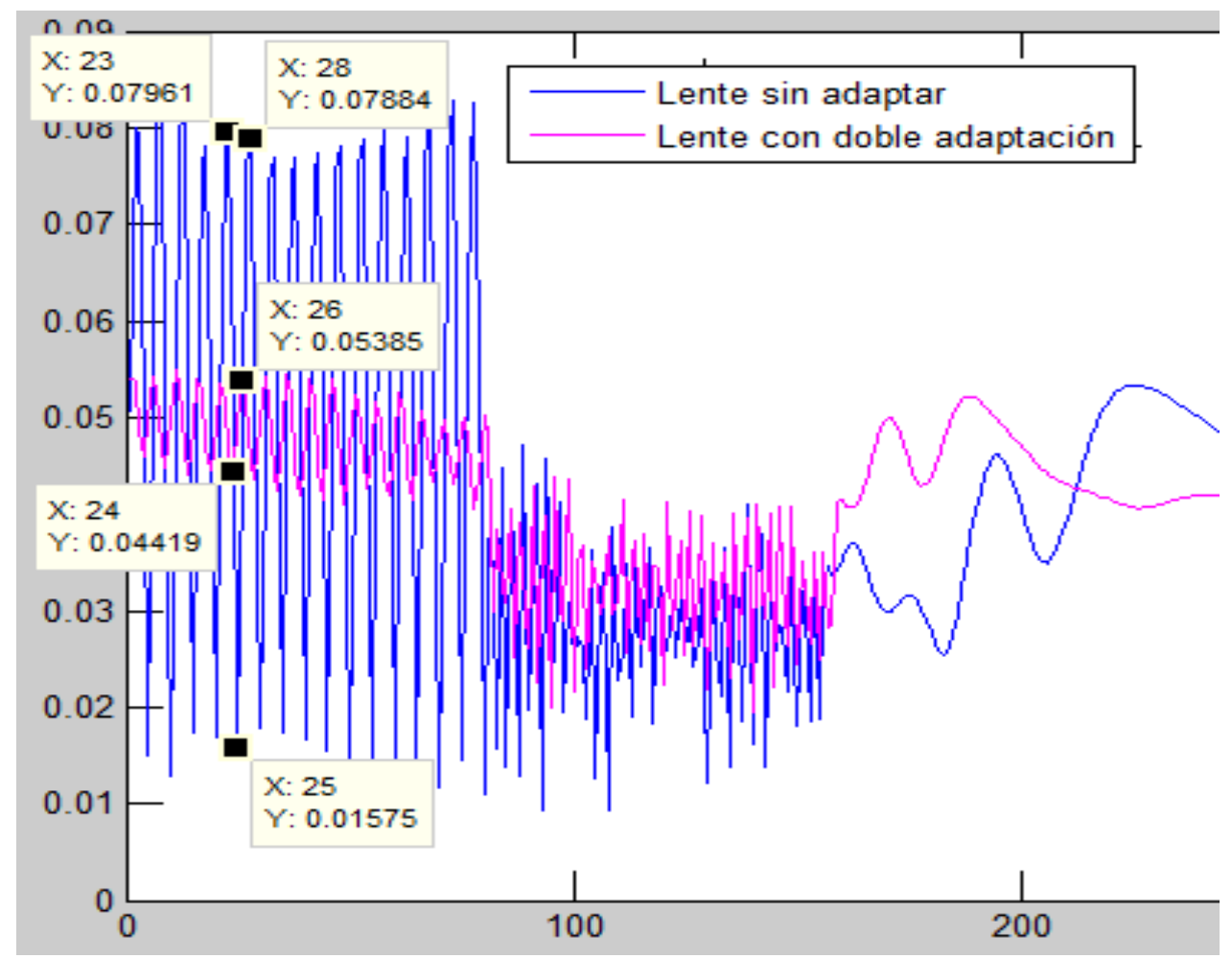

Figura 5.24: Ampliación de la Figura 5.22 b) entre el alimentador y la lente.

A partir de los resultados de campo cercano, Figura 5.24, se mide la distancia entre dos máximos contiguos del campo, siendo ésta de $\lambda 2$, lo cual corresponde a una onda estacionaria generada por la interfase de dos dieléctricos.

A partir de las Figuras 5.23 y 5.24 utilizando la ecuación (5.10) es posible medir la relación de onda estacionaria y por lo tanto el coeficiente de reflexión para la lente, en ambos casos, sin y con los adaptadores. La lente está ubicada entre los puntos 80 y 160 del eje $\mathrm{x}$.

En la Figura 5.25 a) se compara el diagrama de radiación de la antena lente sin adaptar (azul) con la lente adaptada con una sola capa (rojo). Mientras en la Figura 5.25 b) se compara el diagrama de radiación de la antena lente sin adaptar (azul) con la lente adaptada con doble capa (magenta). 


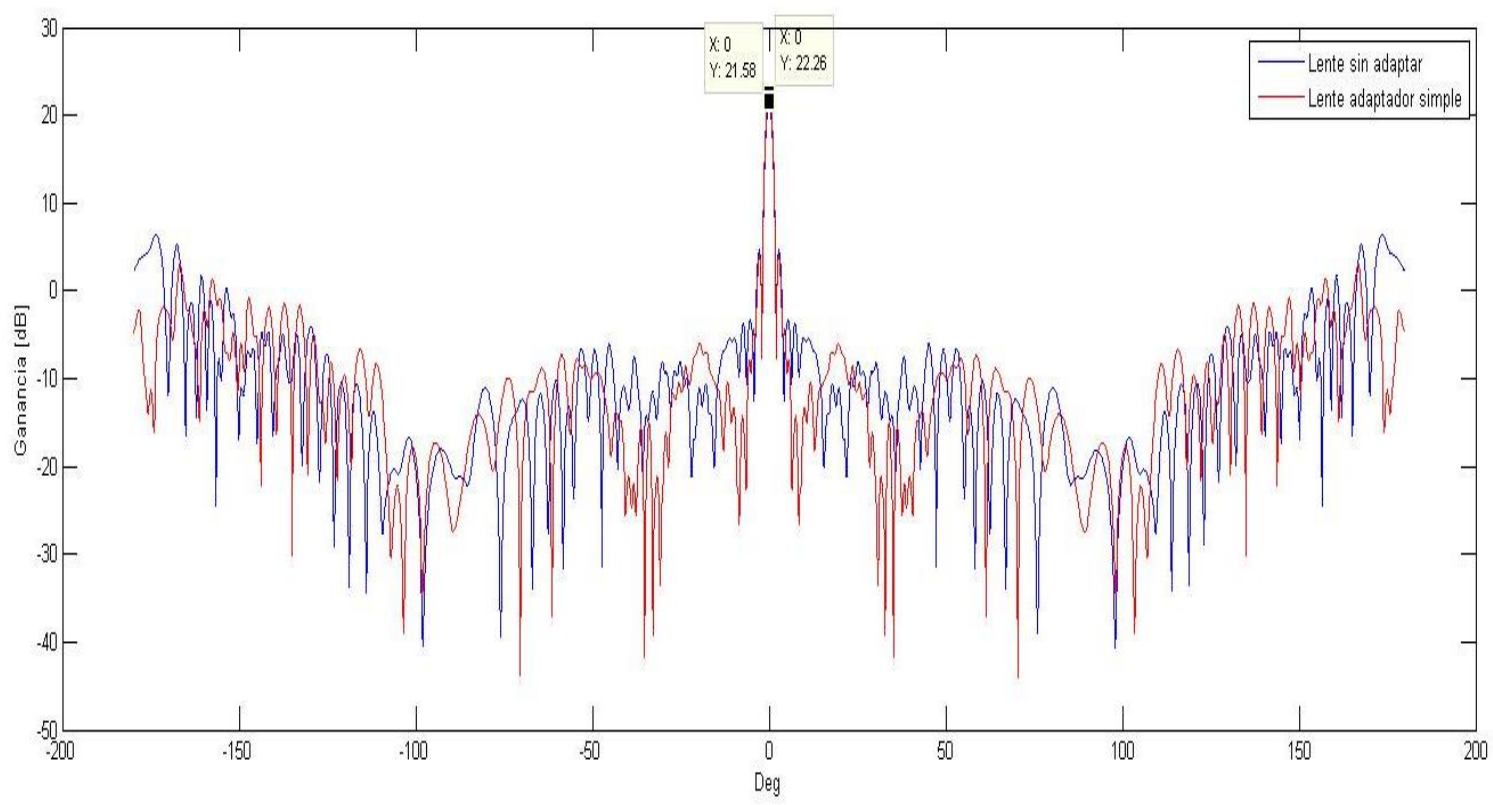

a)

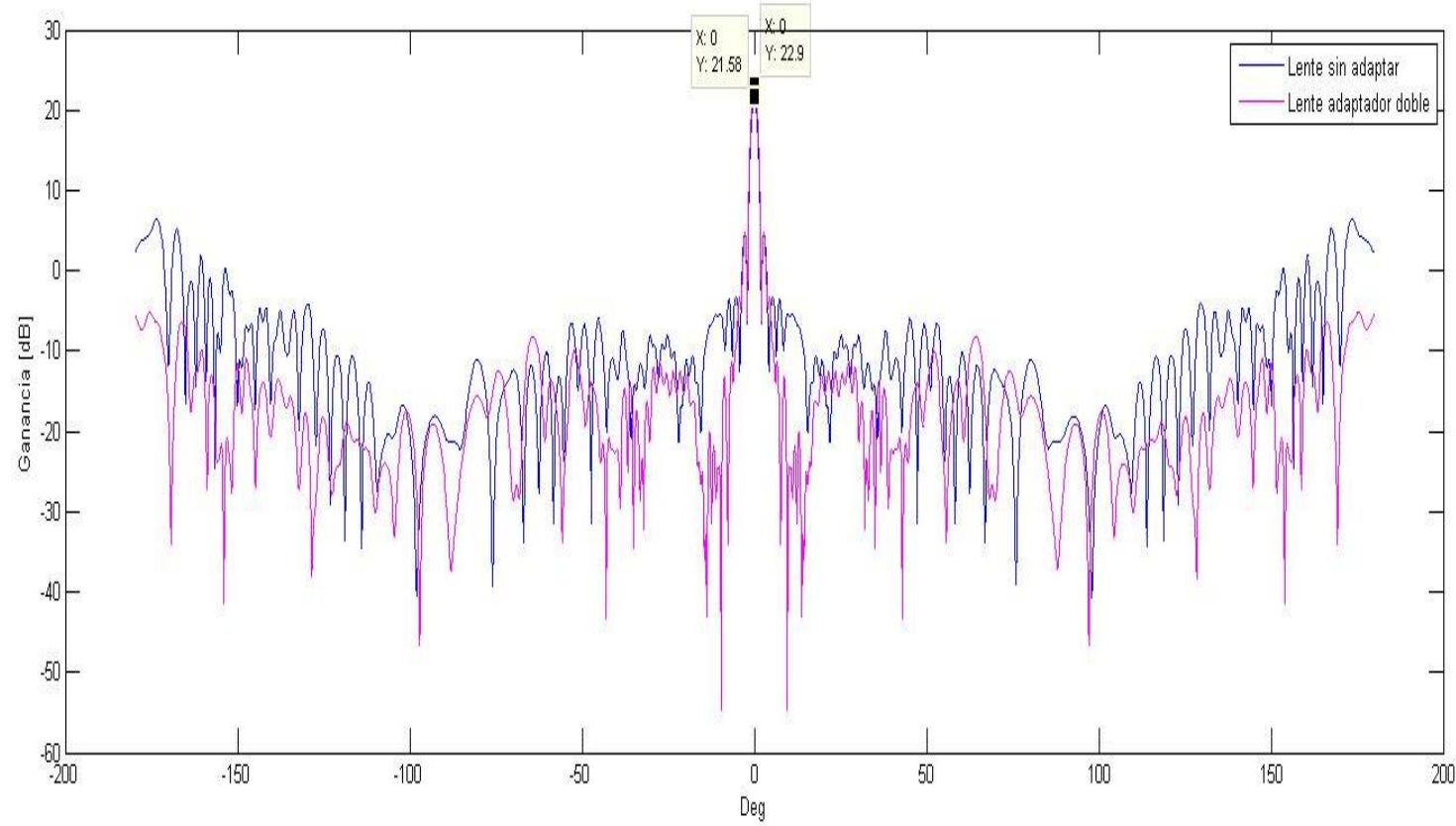

b)

Figura 5.25: a) Diagrama de radiación de la antena lente hiperbólica adaptada con una capa y sin adaptar, b) diagrama de radiación de la antena lente adaptada con doble capa y $\sin$ adaptar.

Del diagrama de radiación se pueden obtener todas las características eléctricas de la antena lente. 
En la Tabla 5.6 se resumen los resultados de la directividad máxima de las antenas lente sin adaptar y adaptadas, así como la relación de onda estacionaria medida entre la lente y el alimentador.

Tabla 5.6: Directividad máxima y ROE para los tres casos estudiados.

\begin{tabular}{|l|l|l|c|}
\hline & \multicolumn{1}{|c|}{$\begin{array}{c}\text { Directividad } \\
{[\mathbf{d B}]}\end{array}$} & ROE & $\begin{array}{c}\text { Tiempo } \\
\text { computacional [s] }\end{array}$ \\
\hline Sin adaptar & 21,58 & 4,65 & 208 \\
\hline Adaptador simple & 22,26 & 1,79 & 225 \\
\hline Adaptador doble & 22,90 & 1,27 & 230 \\
\hline
\end{tabular}

Como se observa en la Tabla 5.6, con la lente adaptada disminuye el ROE, por lo tanto, la onda estacionaria entre el alimentador y la lente. El ROE correspondiente a una adaptación ideal, sin onda reflejada, es uno. Esto conlleva a un aumento en la ganancia de la antena lente adaptada de $0,68 \mathrm{~dB}$ para el adaptador simple y 1,32 dB para el adaptador doble.

Estos valores se pueden comparar con el cálculo aproximado de las pérdidas por reflexión de la lente sin adaptar, que según la ecuación (5.7), para una lente de $\varepsilon_{\mathrm{r}}=4$ resultan de $\mathrm{L}=0,99 \mathrm{~dB}$. Es decir, la capa adaptativa ha logrado compensar en gran medida las pérdidas por reflexión.

Se puede repetir el proceso para lentes de diferentes perfiles. En la figura $5.26 \mathrm{se}$ muestra un diagrama de la intensidad relativa del campo dentro y fuera de una lente de perfil elíptico en el espacio de simulación (cuadrado unidad) para la lente a) sin adaptador y b) doble adaptador. 


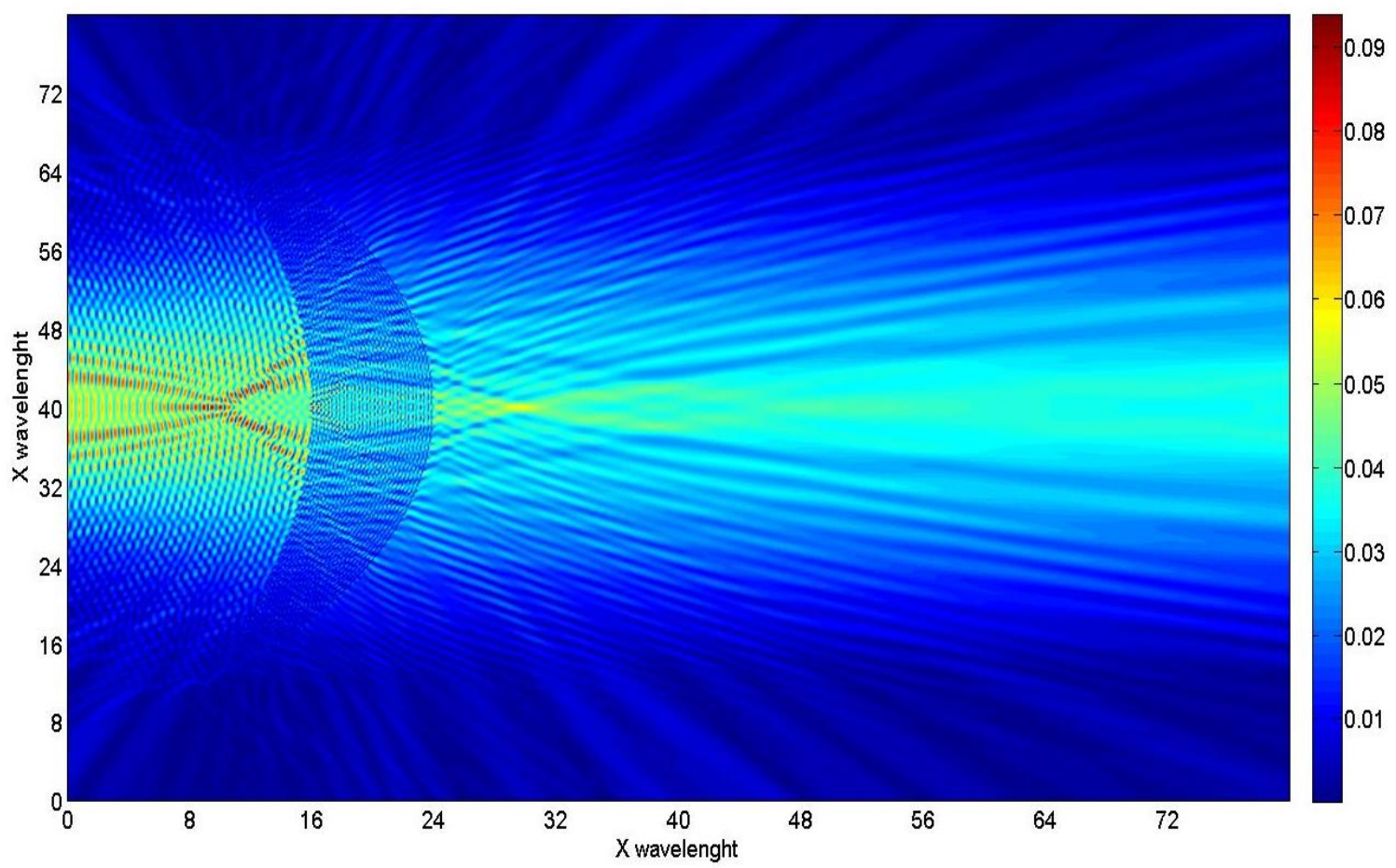

a)

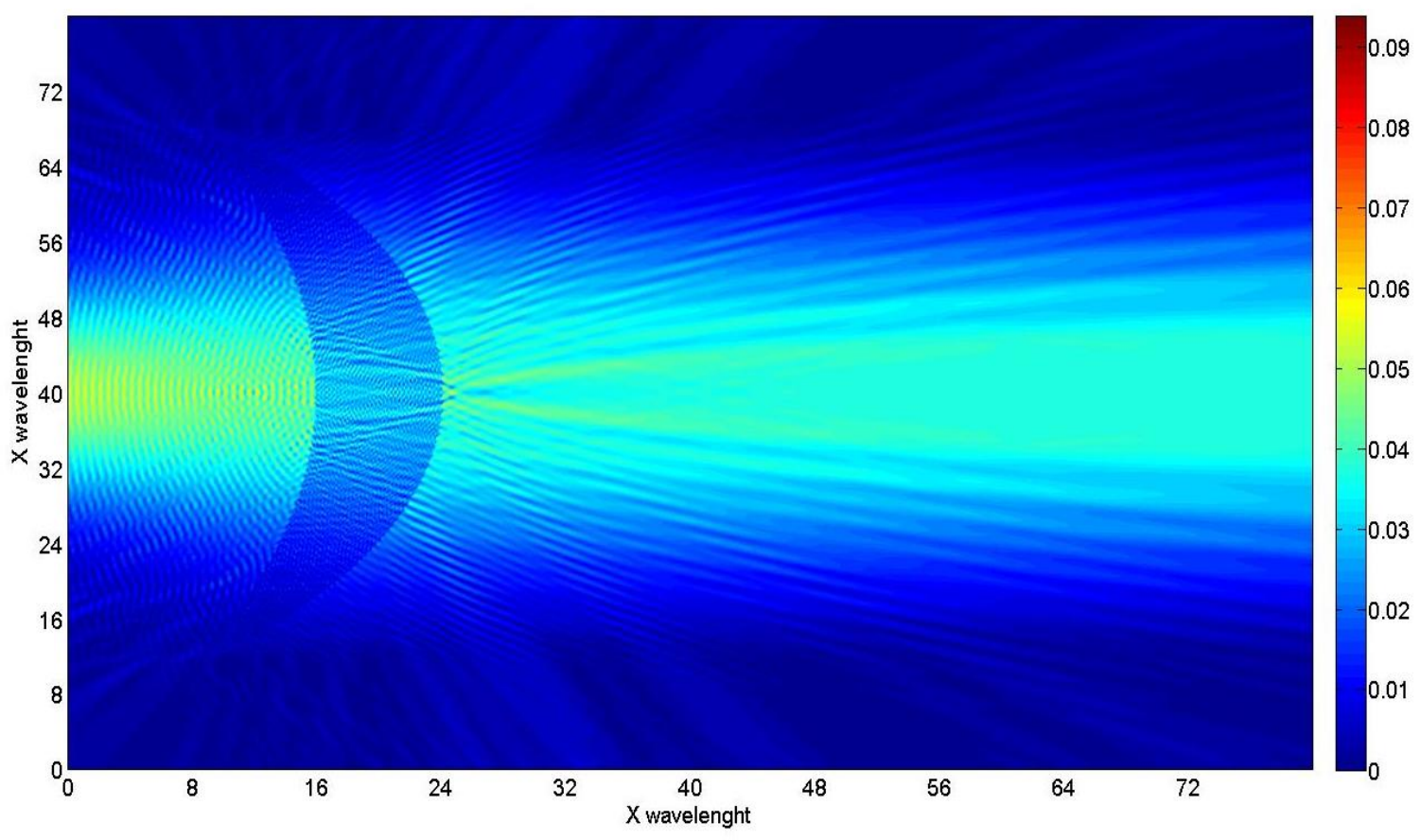

b)

Figura 5.26: Intensidad relativa del campo dentro y fuera de la lente elíptica a) sin adaptar y b) con doble adaptador. 
Al igual que para la lente de perfil hiperbólico, es posible visualizar la onda estacionaria graficando el módulo del campo en el eje de la lente tal como se observa en la Figura 5.27.

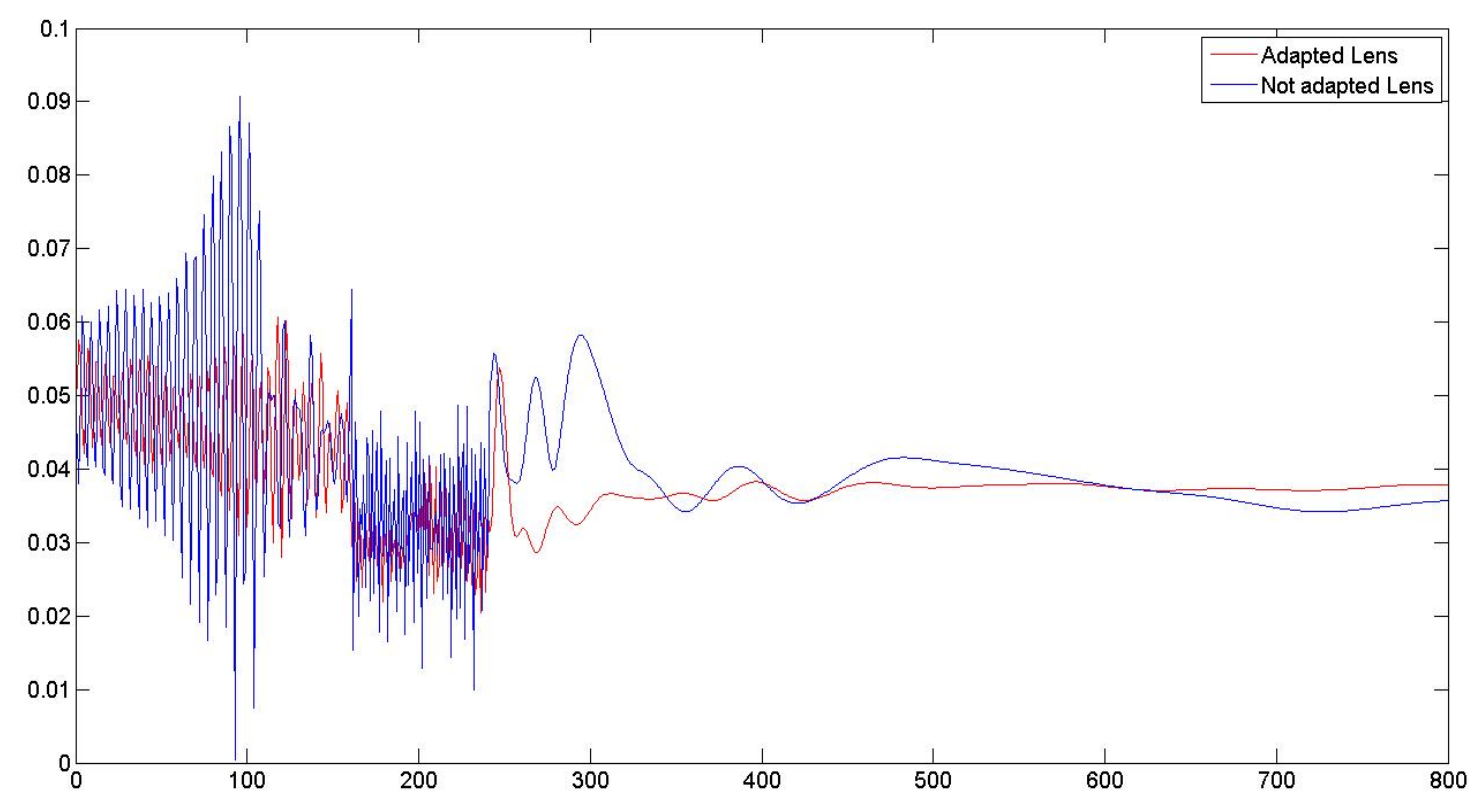

Figura 5.27: Módulo del campo eléctrico en el eje de la lente sin adaptar y adaptada.

A diferencia de la antena lente hiperbólica, la antena lente elíptica presenta un perfil de onda estacionaria con una amplitud normalizada visiblemente variable entre el alimentador y la lente.

La Figura 5.28 muestra los diagramas de radiación para la antena lente elíptica con adaptador y sin adaptador. 


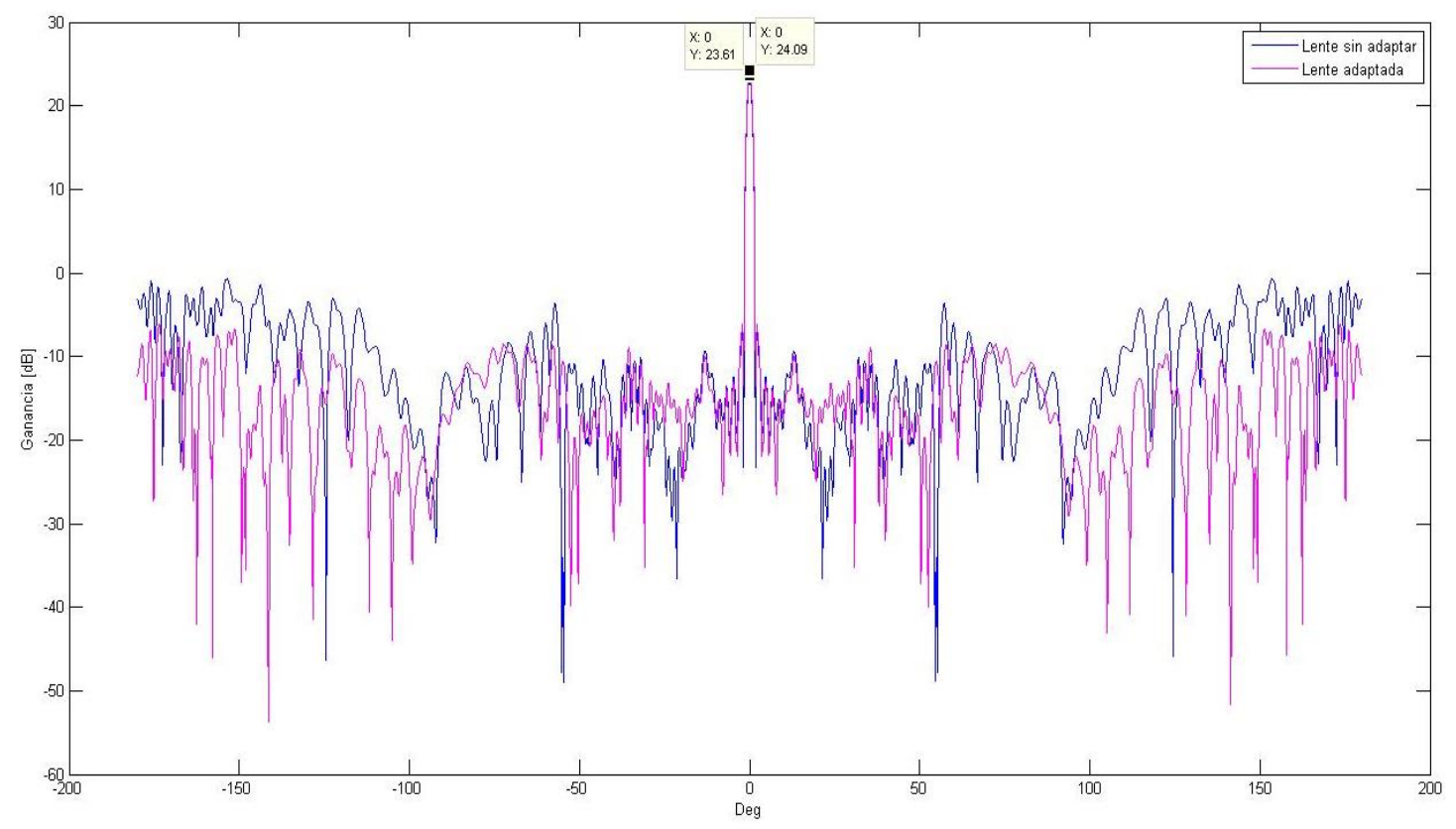

Figura 5.28: Diagrama de radiación de la lente elíptica sin adaptar y adaptada.

La tabla 5.7 resume los datos comparativos de las características de radiación y ROE de las antenas lente hiperbólica y elíptica adaptadas y sin adaptar.

Tabla 5.7: Comparación de las características de radiación y ROE de las antenas lente de perfil Hiperbólico y Elíptico.

\begin{tabular}{|l|l|l|l|l|l|l|l|}
\hline & & Ganancia & $\begin{array}{c}\text { Ancho } \\
\text { de haz }\end{array}$ & NLPS & $\begin{array}{c}\text { front } \\
\text { to } \\
\text { back }\end{array}$ & $\begin{array}{c}\text { ROE } \\
\text { pico }\end{array}$ & $\begin{array}{c}\text { ROE } \\
\text { medio }\end{array}$ \\
\hline Perfil & & G [dB] & $\left.\boldsymbol{\Delta \theta}_{\mathbf{3 d b}} \mathbf{[}^{\mathbf{0}}\right]$ & {$[\mathbf{d B}]$} & [dB] & & \\
\hline Hip. & Sin Adaptar & 21,58 & 1,80 & 16,94 & 19,28 & 7,48 & 5,57 \\
\hline & Adaptada & 22,90 & 1,80 & 18,42 & 28,39 & 1,31 & 1,24 \\
\hline Elíptico & Sin Adaptar & 23,16 & 1,40 & 30,86 & 21,50 & 184,38 & 11,55 \\
\hline & Adaptada & 24,09 & 1,30 & 30,27 & 36,30 & 2,16 & 1,45 \\
\hline
\end{tabular}

Como se observa, las antenas lente de ambos perfiles presentan mejoras en todas las características de radiación para la lente adaptada. Asimismo, es notable la disminución de la relación de onda estacionaria

Los resultados obtenidos, permiten observar el efecto en las características eléctricas de las antenas lentes dieléctricas de la aplicación de capas adaptativas de cuarto de onda con el fin de reducir las pérdidas por reflexión. Asimismo, se pudo observar y medir en campo cercano la relación de onda estacionaria entre la lente y el alimentador 
para la antena lente adaptada y sin adaptar, situación solo observable mediante simulaciones por métodos full-wave [81].

\subsection{Simulaciones de lentes mediante software comercial}

Con el fin de comparar resultados de simulaciones utilizando métodos full-wave y métodos asintóticos se aplica el software comercial Grasp 10.6.0 ya que dicho software permite simular con ambos métodos.

Para ello se propone la simulación de un ejemplo de empleo de antenas lente para telescopios refractivos.

Los telescopios refractivos son una alternativa atractiva a los telescopios reflectantes, ya que pueden proporcionar una polarización cruzada baja y capacidades de escaneo más amplias en un diseño mucho más compacto. Los telescopios refractivos están hechos típicamente de lentes dieléctricas grandes simétricas circulares, con un eje de rotación común. Las lentes a menudo están recubiertas con material antirreflectante para reducir las reflexiones no deseadas de las lentes y así mejorar la eficiencia del telescopio.

Las lentes dieléctricas para telescopios refractivos se diseñan comúnmente con óptica geométrica (GO) u óptica física (PO). Sin embargo, para un análisis preciso de RF del sistema completo, las formulaciones tradicionales de GO y PO no son lo suficientemente precisas ya que no tienen en cuenta las reflexiones dentro de la lente. Además, como ya se detalló previamente, las formulaciones tradicionales de GO y PO tienen dificultades para modelar el material de recubrimiento. Finalmente, si se consideran múltiples lentes, el acoplamiento mutuo entre las lentes puede ser una tarea lenta con PO y GO.

Una solución full-wave es el método más preciso para calcular el rendimiento de RF de un sistema refractivo. Cuando el sistema es rotacionalmente simétrico, un método de momentos del cuerpo de revolución (BoR-MoM) puede ser una muy buena opción en términos de tiempo de cálculo [53]. El solucionador BoR-MoM de orden superior disponible comercialmente en el complemento MoM para GRASP es el solucionador más preciso y eficiente para lentes dieléctricas recubiertas en el mercado analizado hasta el momento de escritura de la presente Tesis doctoral. 


\subsubsection{Simulaciones full-wave mediante el software comercial Grasp}

A modo de ejemplo se diseña y analiza una lente dieléctrica de perfil Hiperbólico biconvexa recubierta con un adaptador. El análisis se realiza con el solucionador BoRMoM, que pertenece al complemento MoM para GRASP.

La lente está iluminada por un haz gaussiano a una frecuencia de $100 \mathrm{GHz}$. Cuenta con un diámetro de $286 \mathrm{~mm}$ (95 $\lambda$ ), e iluminada en su cara izquierda ubicado a $385 \mathrm{~mm}$ de la lente, como se muestra en la Figura 5.26. La lente está hecha de polietileno de peso molecular ultra alto (UHMW-PE) con un índice de refracción de 1.512, y está recubierta en ambas caras con una capa de $0.609 \mathrm{~mm}$ de politetrafluoroetileno (PTFE) poroso.

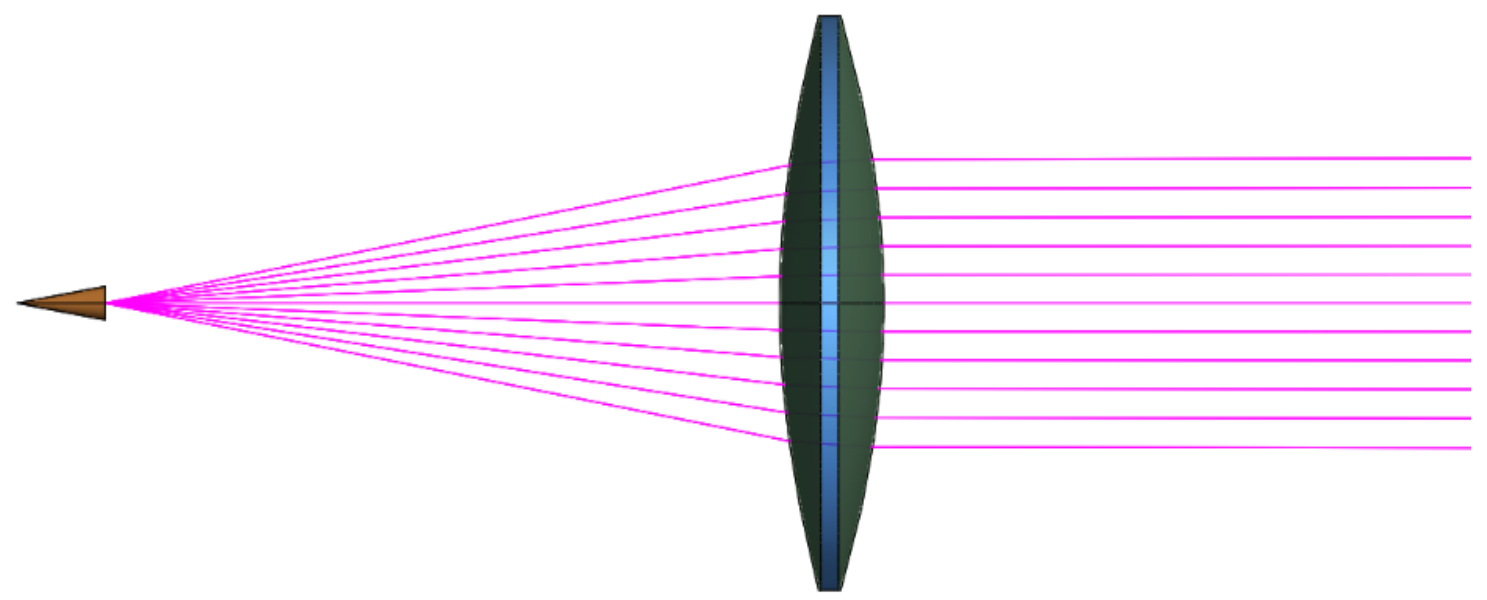

Figura 5.29: Antena lente hiperbólica biconvexa diseñada con software Grasp 10.6.0.

El campo se calcula en un plano a una distancia de $550 \mathrm{~mm}$ del alimentador, después de haber pasado la lente. El plano se resalta en azul claro en el diagrama de la Figura 5.30. 


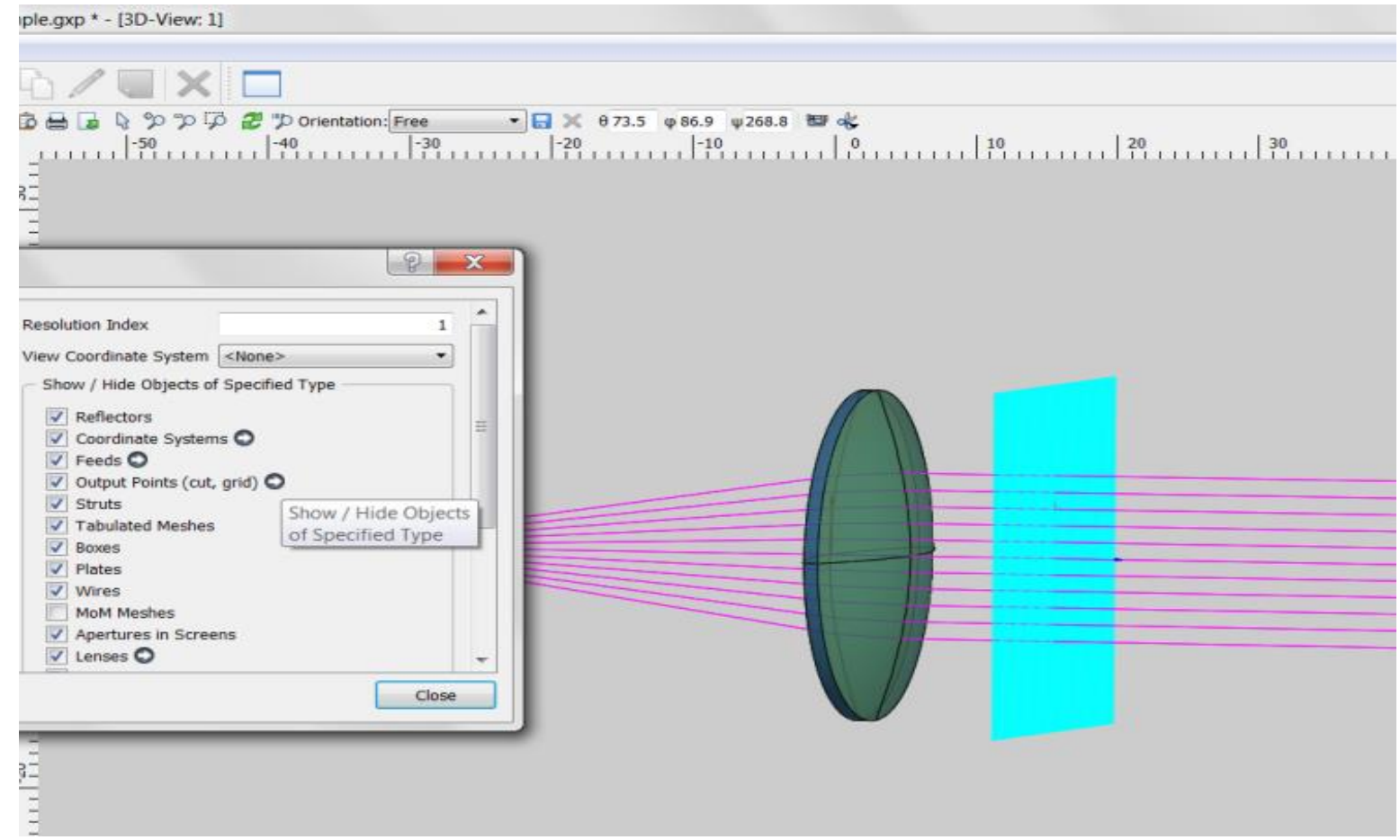

Figura 5.30: Vista 3D de la antena lente hiperbólica biconvexa simulada.

El campo calculado mediante BoR-MoM en un plano a una distancia de $550 \mathrm{~mm}$ de la alimentación se ve en la gráfica de la Figura 5.31. Se pueden observar pequeñas ondulaciones en los patrones del campo lejano debido a múltiples reflejos dentro de la lente. 


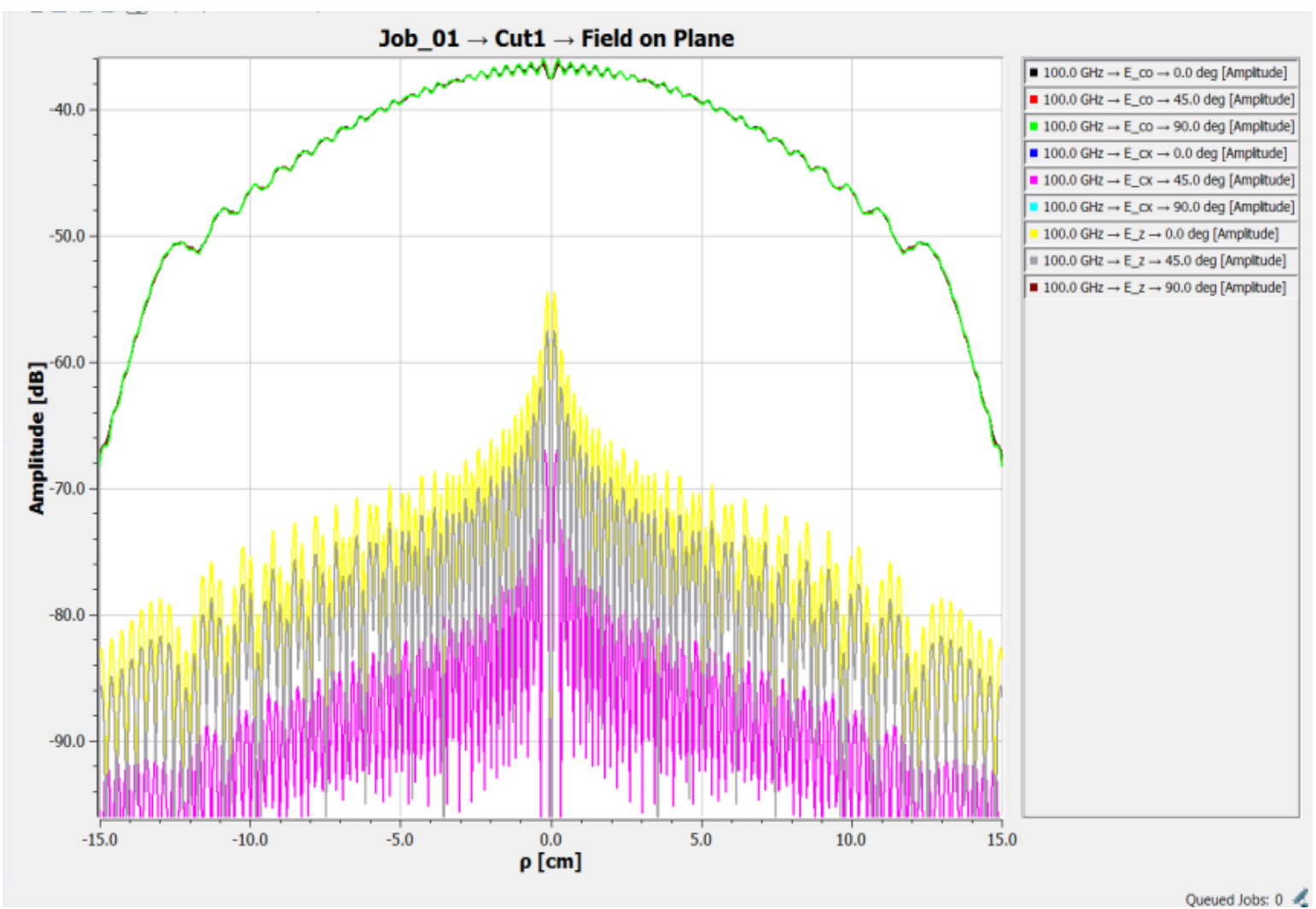

Figura 5.31: Resultado del análisis BoR-MoM de la lente hiperbólica biconvexa recubierta.

El tiempo de simulación de la lente fue de 11 segundos.

\subsubsection{Simulaciones con técnicas asintóticas mediante el software comercial Grasp}

Para analizar la lente mediante métodos más tradicionales tales como GO-PO, primero se elimina la capa de recubrimiento de la lente. Para las mismas características del ejemplo anterior (frecuencia, tamaño de la lente, etc), se simula la lente y los resultados se muestran en la Figura 5.32.

Al comparar los resultados con la simulación full-wave, se observa que GO-PO no puede reproducir los patrones (ripples) debido a las reflexiones dentro de la lente tal como se observan en los resultados mediante BoR-MoM.

Finalmente se observa que GO-PO tampoco está de acuerdo con BoR-MoM para la componente contrapolar del campo. Una conclusión importante es que a medida que aumenta el índice de refracción de la lente, los ripples se vuelven cada vez más pronunciados y dicho efecto no puede ser reproducido por GO-PO. 


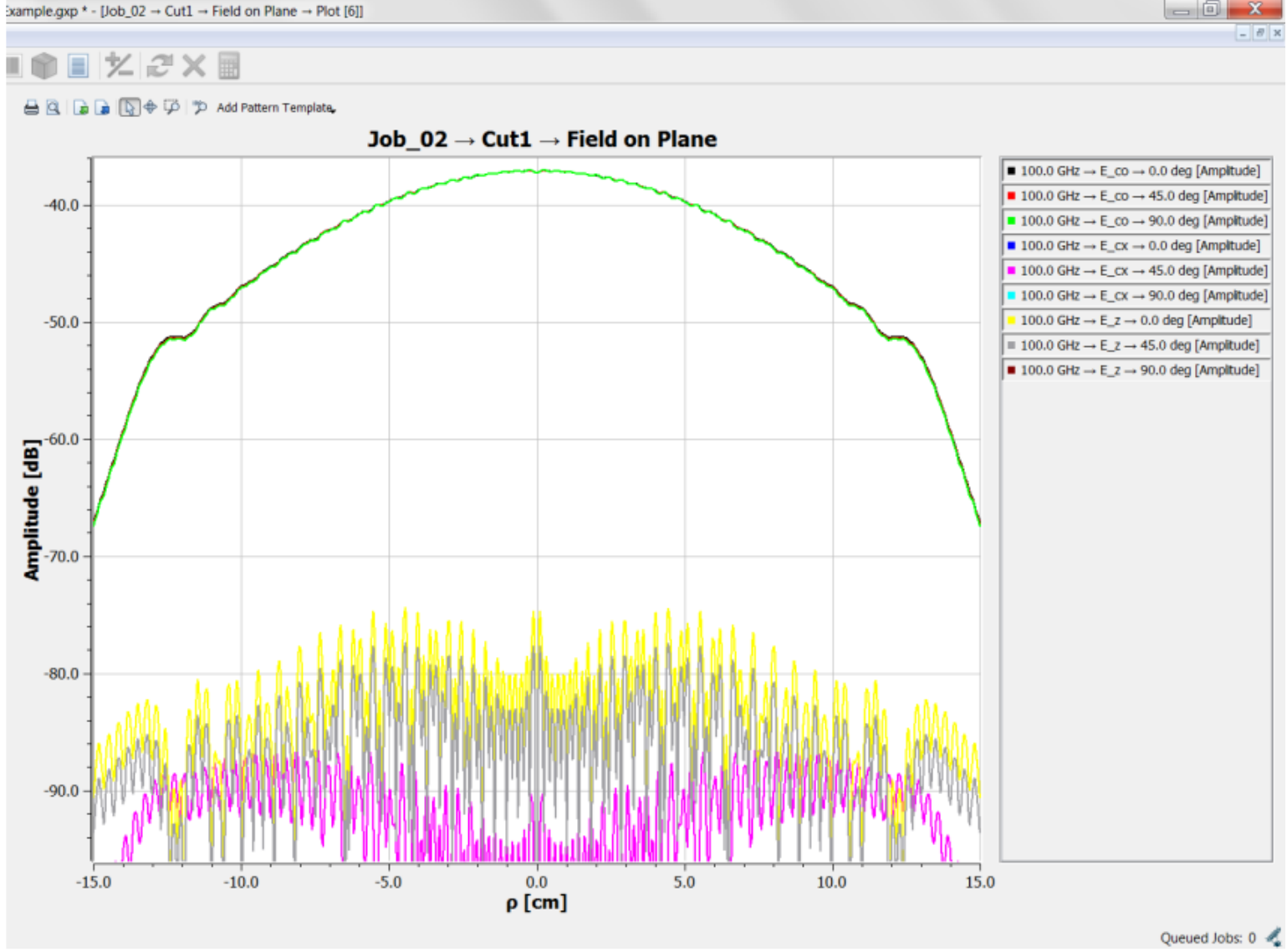

Figura 5.32: Resultado del análisis GO-PO de la lente hiperbólica biconvexa no adaptada.

El tiempo de simulación de la lente fue de 9 segundos. 


\section{CAPÍTULO 6}

\section{Antenas lente Planas}

\subsection{Introducción}

Como se estudió en el Capítulo 3, la directividad de las antenas lente, al igual que otras antenas de apertura, depende de la relación de su diámetro con la longitud de onda aplicada. Para lograr una alta directividad se requiere de diámetros de lente importantes, lo que redunda en un aumento considerable del tamaño y por lo tanto del peso de la lente.

Las lentes dieléctricas homogéneas, vistas en el Capítulo 4, tienen un espesor variable, que define su perfil, con el fin de igualar a la salida el ángulo de fase del campo electromagnético de modo de obtener una onda plana. Dichas lentes proporcionan alta eficiencia y ancho de banda, pero sufren de ser pesadas, voluminosas y caras [46].

Un tipo de lente que gana terreno desde mediados de la década de 1990, y que emplea menos material y por lo tanto puede poseer menor peso, es el de la lente plana [82]. Estas lentes también se han denominado superficies selectivas de frecuencia [40, 44, 45], superficies de desplazamiento de fase [30 ,42, 43, 83, 84], matrices de transmisión [85] y antenas - filtro - antenas [86].

Las antenas lente planas son estructuras muy atractivas dentro de las microondas y de las ondas milimétricas, no sólo por su menor peso sino debido a su robustez, su facilidad de construcción actual y su notable repetitividad en su fabricación.

En este capítulo se presentan dos tipos de lentes planas. La lente plana dieléctrica y la lente de zonas de Fresnel. Estas antenas lente fueron simuladas con el software comercial FEKO y un modelo experimental fue construido con el fin de evaluar sus resultados. Para estas simulaciones no fue posible aplicar el código full-wave utilizado para el diseño de antenas lente dieléctricas homogéneas (Capítulo 5) pues no permite simular materiales conductores como los empleados, por ejemplo, en la lente de zonas de Fresnel.

\subsection{Lentes planas o superficies de cambio de fase}

El objetivo de las lentes planas, al igual que las lentes de perfiles cónicos, es modificar la fase de la onda esférica proveniente del alimentador de modo de obtener ondas planas a la salida de la lente. La Figura 6.1 muestra el funcionamiento de una lente dieléctrica (a) y de una lente plana (b). 


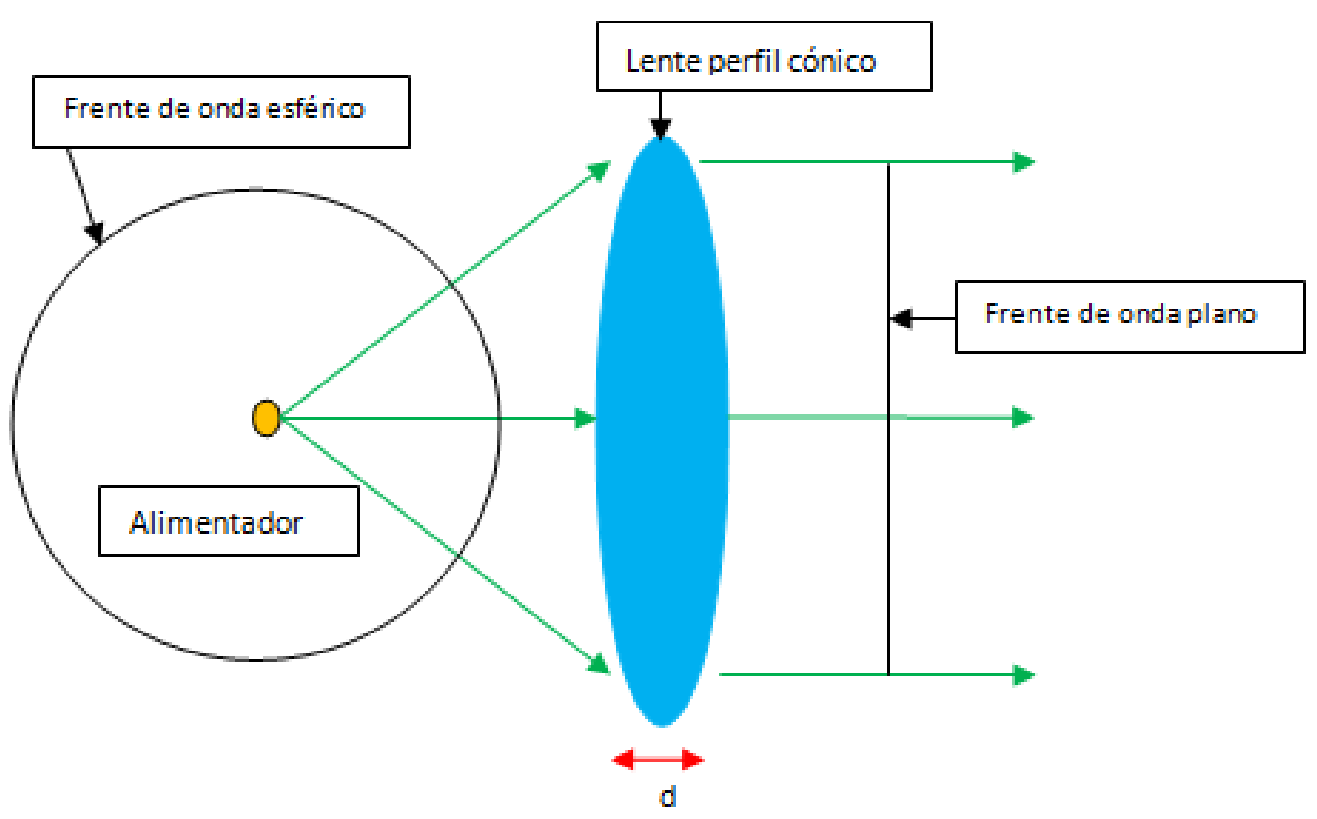

a)

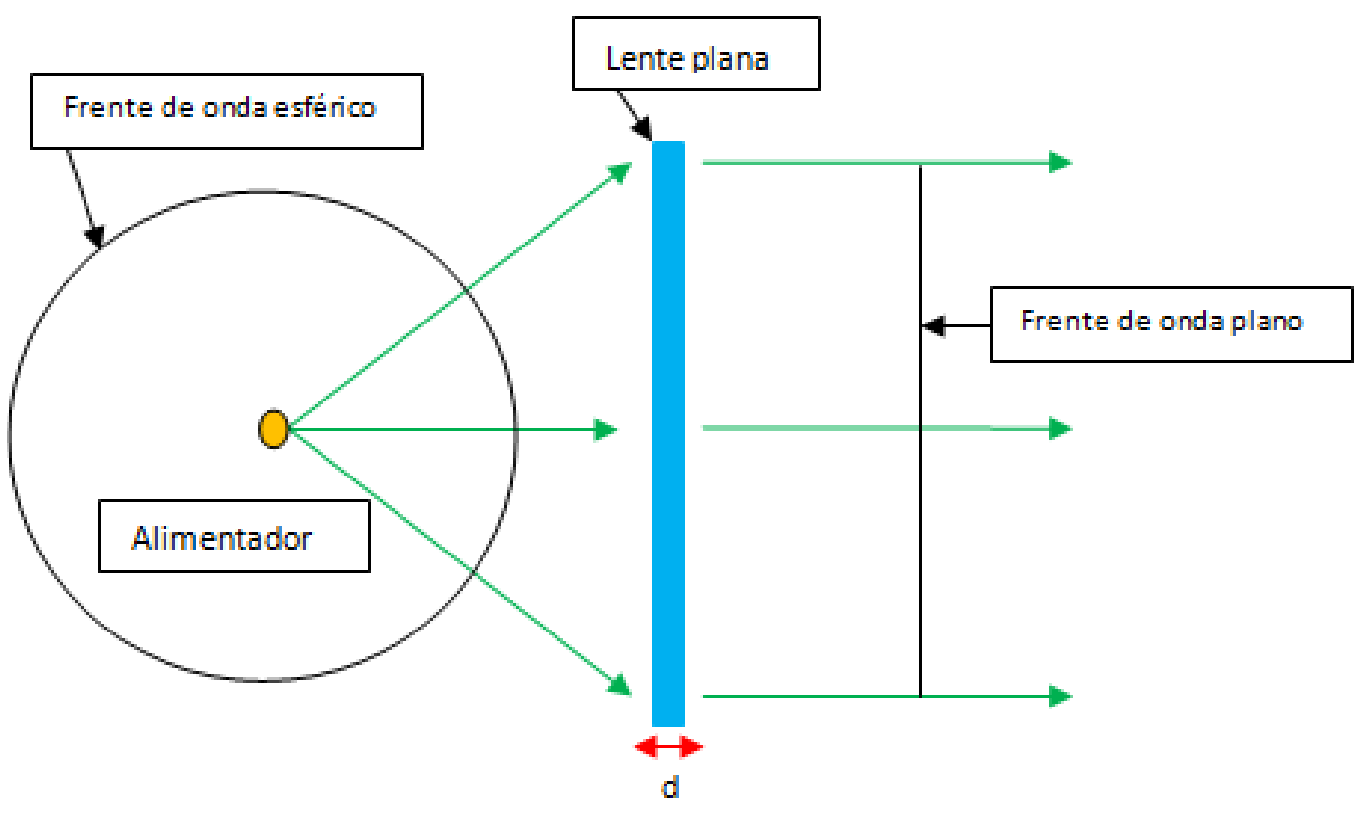

b)

Figura 6.1: Esquema de antena a) lente dieléctrica biconvexa y b) lente plana.

El cambio de fase necesario para obtener una onda plana en una lente plana se puede lograr de muchas maneras [46].

Una lente plana dieléctrica puede diseñarse a partir de modificar la permitividad dieléctrica del material de forma gradual de modo de generar el cambio de fase en cada punto para obtener la onda plana [87].

Otro tipo de lente plana en conocido como "Transmitarray". Dicha lente comprende una superficie de elementos radiantes discretos configurados para producir una distribución de fase que exhibe un efecto de colimación. Los transmitarray 
generalmente están compuestos por una matriz plana de celdas unitarias de desplazamiento de fase iluminadas por una fuente colocada a una distancia focal $\mathrm{f}$ de la matriz (Figura 6.2) [88]. Las celdas unitarias están diseñadas para generar una distribución de fase adecuada en la apertura de la matriz para enfocar el haz en una dirección específica. Un transmitarray se puede fabricar utilizando tecnologías de placa de circuito impreso estándar con materiales de baja pérdida, lo que permite bajos costos de fabricación y altos rendimientos.

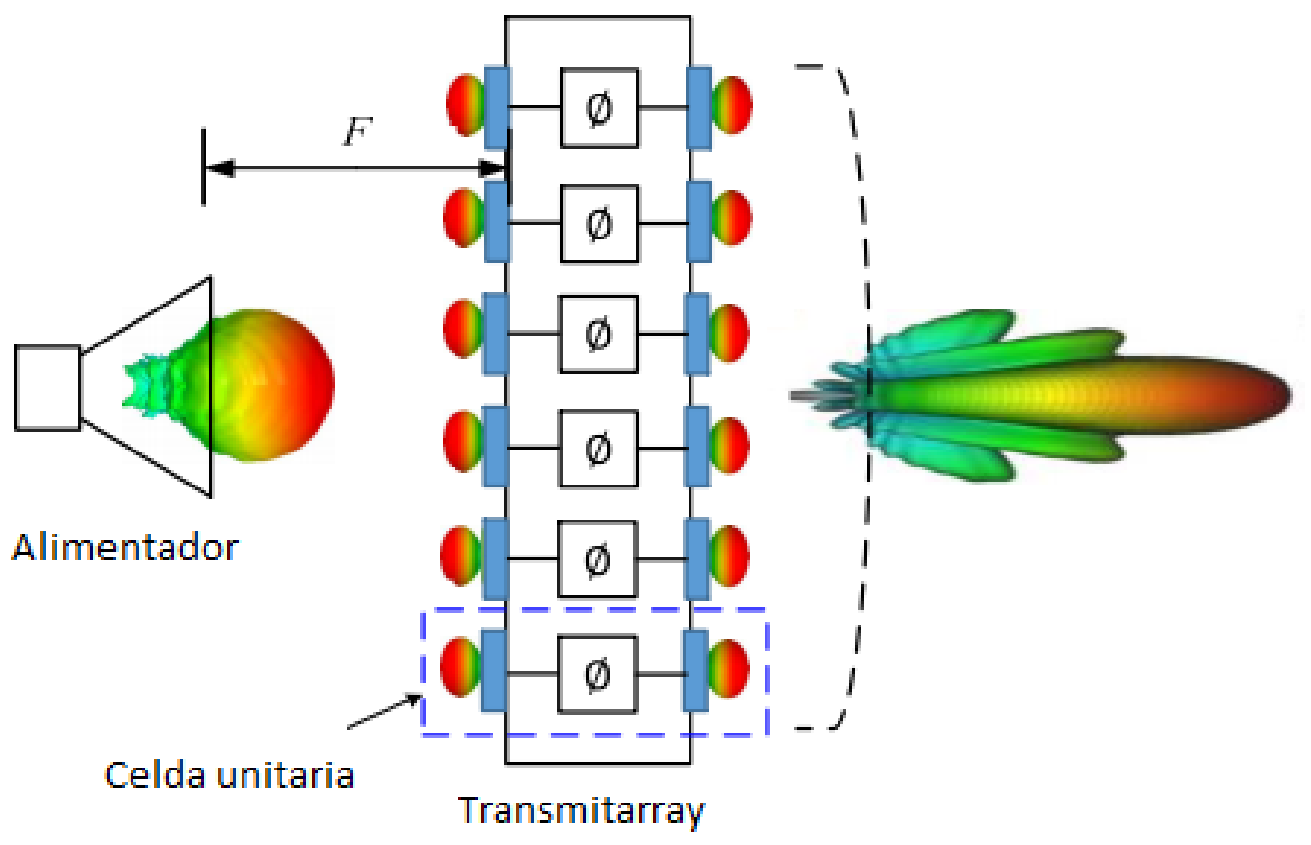

Figura 6.2: Transmitarray.

Un tipo de transmitarray es la denominada lente de zonas de Fresnel.

\subsection{Lentes planas dieléctricas}

Como se mencionó previamente, una antena plana puede fabricarse haciendo variar su permitividad dieléctrica (épsilon relativo) para generar el cambio de fase $(\beta)$ siguiendo el comportamiento de una lente dieléctrica. 


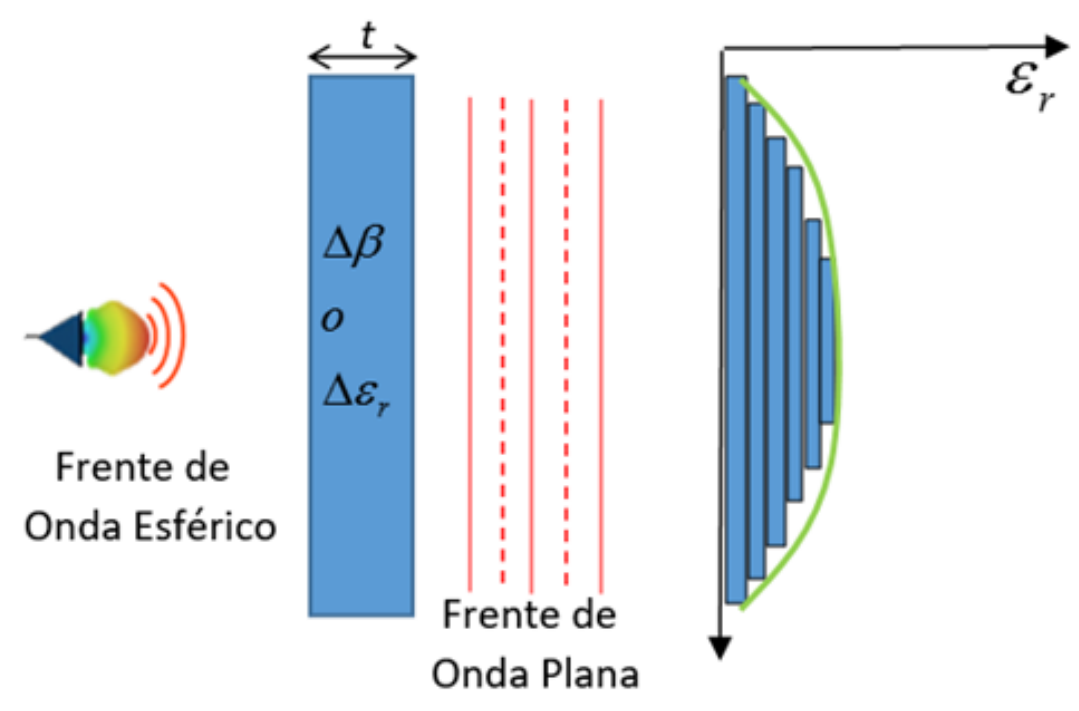

Figura 6.3: Antena lente plana dieléctrica y variación de la permitividad de la lente plana.

La construcción de una lente plana con un perfil radial de cambio de fase continuo es de dificultosa concreción. Una alternativa es discretizar en pasos los valores de cambio de fase a fin de conseguir un cambio de fase radial que siga al perfil de una lente biconvexa como se observa en la figura 6.4.

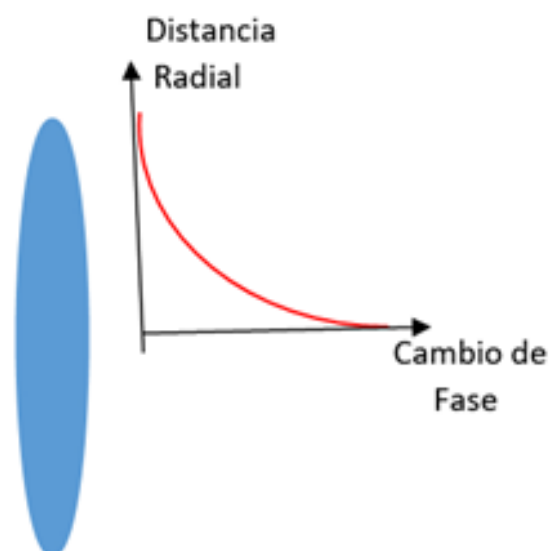

LENTE BICONVEXA

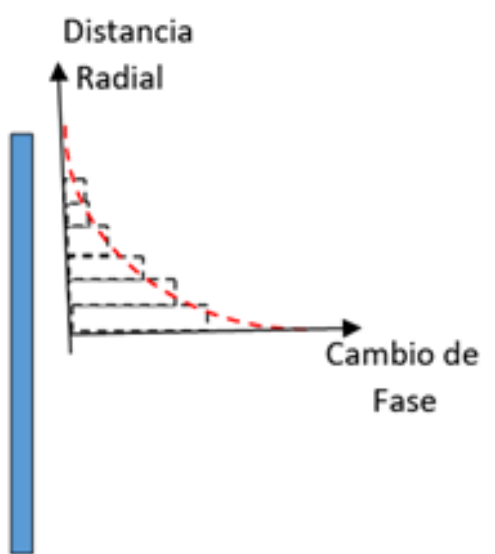

LENTE PLANA

a)

b)

Figura 6.4: Cambio de fase a) Lente biconvexa y b) lente plana equivalente.

En la Figura 6.5 se observa una lente discretizada en seis regiones. 


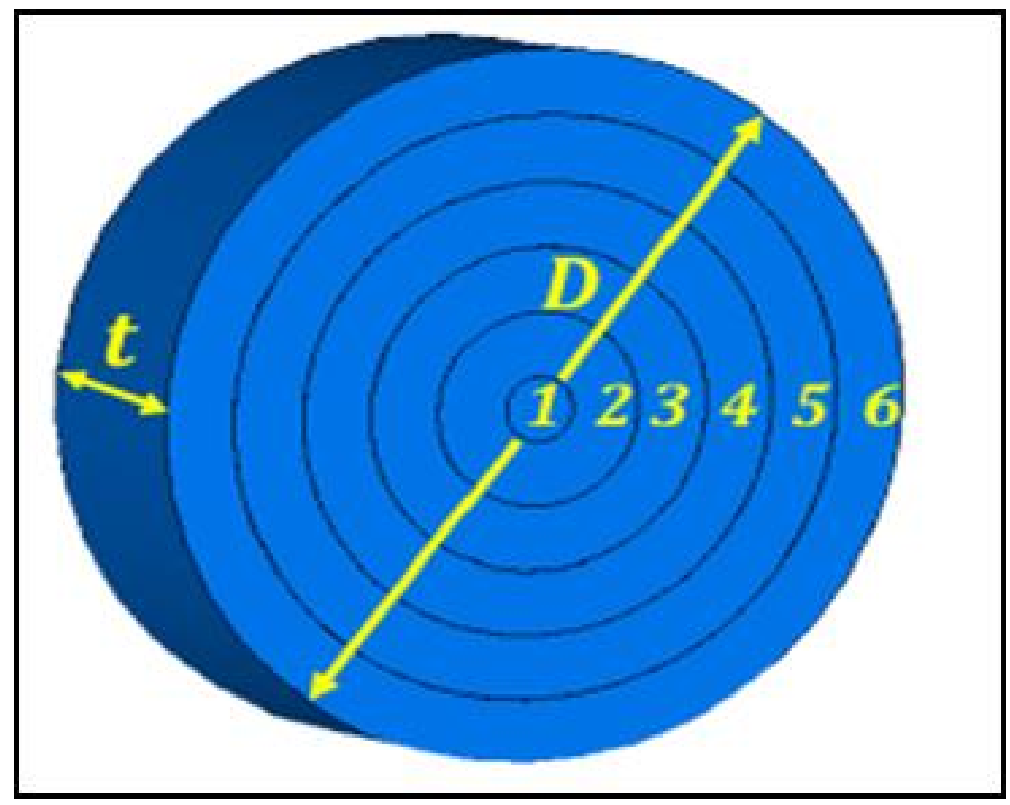

Figura 6.5: Lente plana discretizada.

Debido a que la construcción de una lente no homogénea discretizada (diferentes constantes dieléctricas) implicaría diferentes materiales y métodos de fabricación complejos, su concreción también presenta dificultades. Una alternativa posible resulta de modificar la contante dieléctrica de un material homogéneo a partir de generar pequeños orificios en el sustrato, de modo que la composición del material con el aire modifique su constante dieléctrica efectiva. En la Figura 6.6 se observa una lente plana discretizada, donde en cada región se logra una diferente permitividad a partir de una dada distribución de las perforaciones.

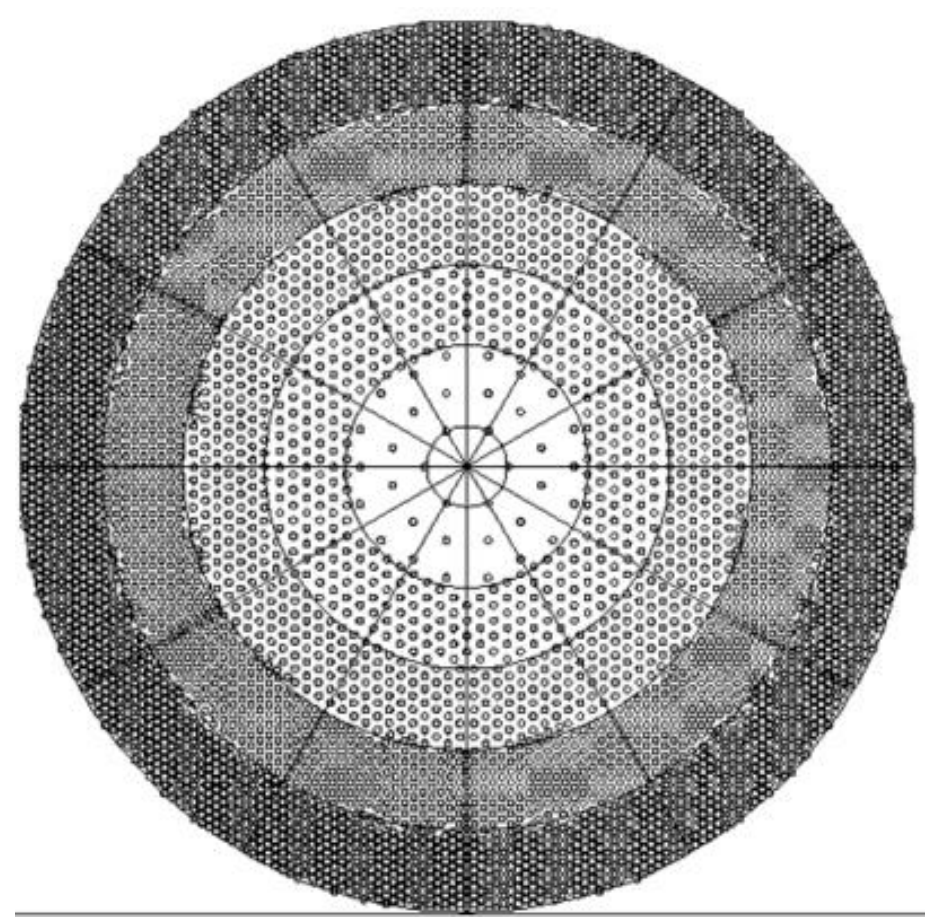

Figura 6.6: Lente plana con orificios. 
La permitividad efectiva de cada región puede ser controlada por el diámetro de los orificios y su separación: cuanto mayor sea el diámetro y menor su separación, menor será la permitividad efectiva para un material de sustrato dado.

Si el diámetro de los orificios y la separación entre ellos se mantienen pequeños en comparación con la longitud de onda de funcionamiento, la permitividad relativa efectiva del sustrato perforado puede asumirse como uniforme, en cada región discretizada de la lente.

\subsubsection{Diseño de una lente plana dieléctrica}

Para diseñar la lente perforada el primer paso es discretizar la lente deseada. La Figura 6.7 muestra el perfil de una lente discretizada en 6 regiones.

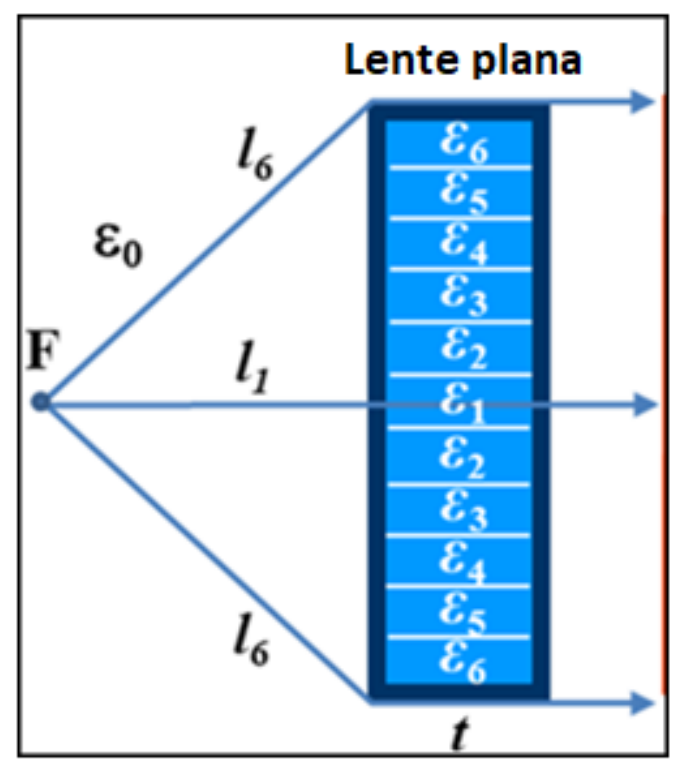

Figura 6.7: Diseño lente discretizada.

El cambio de fase de la onda que atraviesa la lente en cada región discretizada de la misma puede expresarse como:

$$
\beta=k \cdot t=\frac{2 \pi}{\lambda} t=\frac{2 \pi}{\lambda_{0}} \sqrt{\varepsilon_{r}} t
$$

donde $k$ es el número de onda, $t$ es el espesor de la lente plana, $\lambda$ es la longitud de onda en el material, $\lambda_{0}$ es la longitud de onda en el aire y $\varepsilon_{r}$ es la permitividad o constante dieléctrica relativa del material.

Por lo tanto, el cambio de fase en una lente plana se puede lograr, según lo expresado en la ecuación (6.1), haciendo variar la constante dieléctrica para cada región. 
Igualando la fase a la salida de la lente se debe cumplir que el cambio de fase en la parte superior de la lente sea igual al cambio de fase en el centro de la lente tal como se observa de la 6.7 .

$$
k_{0} l_{1}+k_{1} t=k_{0} l_{6}+k_{6} t
$$

De (6.2) se puede obtener la contante dieléctrica en el centro de la lente.

$$
\varepsilon_{r_{1}}=\left(\frac{l_{6}-l_{1}+\sqrt{\varepsilon_{r_{6}}} t}{t}\right)^{2}
$$

Y de la misma forma se puede obtener la permitividad en cada región de la lente discretizada.

Una vez obtenido la permitividad dieléctrica de cada región se puede lograr dicha permitividad mediante perforaciones en el material.

Estas perforaciones se pueden implementar como una rejilla uniforme de orificios en el sustrato mediante un enrejado cuadricular o un enrejado triangular tal como se observa en las Figuras 6.8 a) y b) [87, 89].

La permitividad relativa efectiva de cada región puede determinarse por:

$$
\varepsilon_{\text {eff }}=\varepsilon_{r}(1-\alpha)+\alpha
$$

donde $\alpha$ es el factor de llenado dado por:

$$
\begin{aligned}
& \alpha=\frac{A_{0}}{2 A}, \text { enrejado triangular } \\
& \alpha=\frac{A_{0}}{A}, \text { enrejado cuadrado }
\end{aligned}
$$

donde $\mathrm{A}_{0}$ es el área del orificio perforado, y A es el área correspondiente a la célula unitaria (Figura 6.8). 


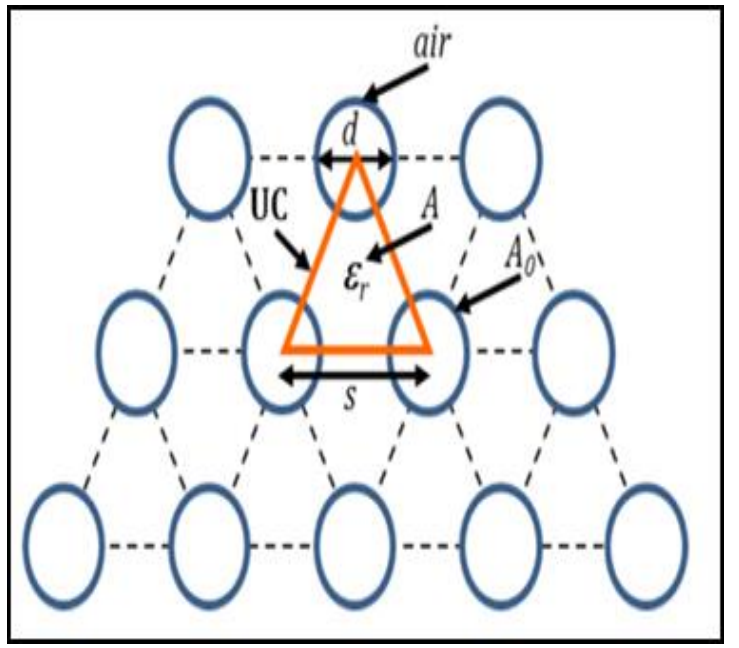

a)

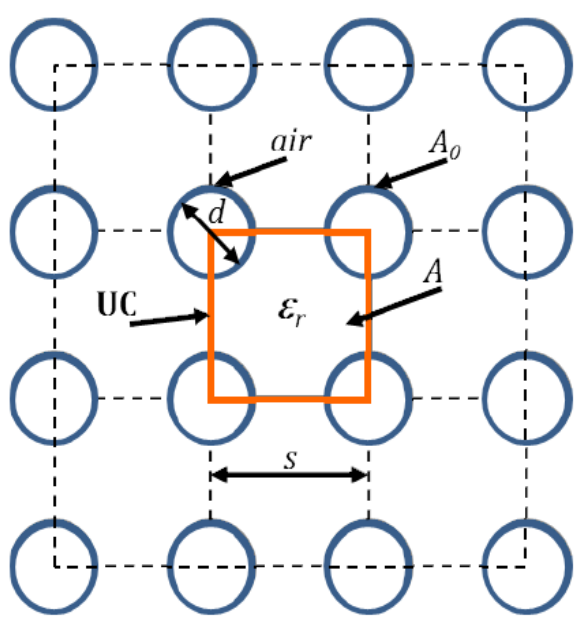

b)

Figura 6.8: Enrejado a) triangular, b) cuadricular.

Por lo tanto, para el enrejado triangular, esto se traduce en:

$$
\alpha=\frac{\pi d^{2} / 4}{2(\sqrt{3} / 4) s^{2}}=\frac{\pi}{2 \sqrt{3}}\left(\frac{d}{s}\right)^{2}
$$

Para el enrejado cuadricular:

$$
\alpha=\frac{\pi d^{2} / 4}{s^{2}}=\frac{\pi}{4}\left(\frac{d}{s}\right)^{2}
$$

\subsubsection{Simulaciones de antena lente plana dieléctrica}

Se simuló una antena lente plana dieléctrica en la frecuencia de $23 \mathrm{GHz}$, con una bocina corrugada como alimentador primario. Las simulaciones se realizaron con el software Feko. La bocina corrugada de la simulación fue diseñada a partir de una bocina real, la cual fue validada mediante mediciones experimentales. 


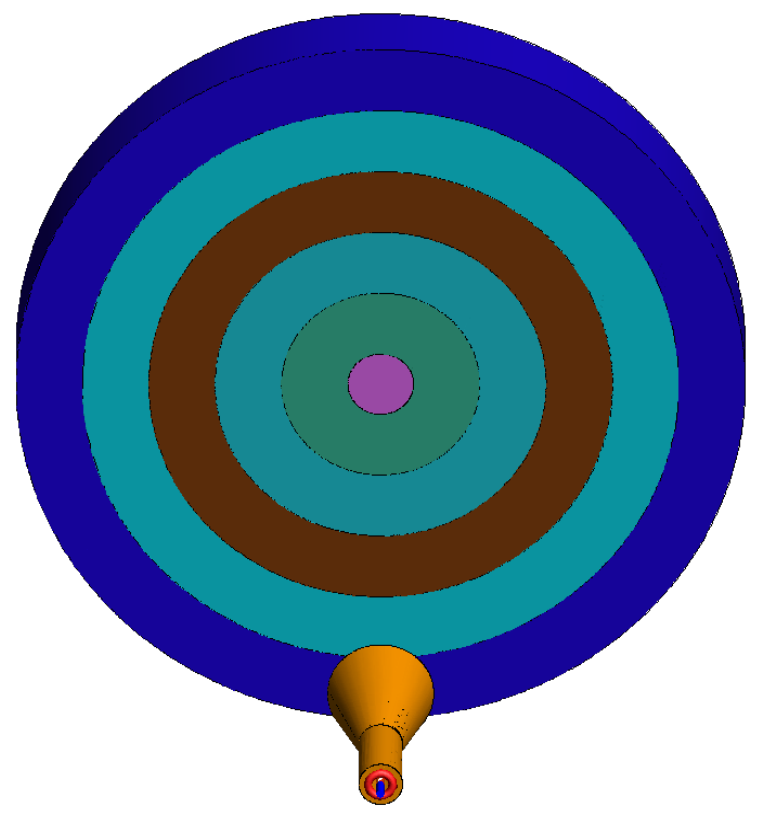

Figura 6.9: Modelo para la simulación de antena plana dieléctrica discretizada realizado mediante el software Feko.

La lente se diseñó a partir de un sustrato de teflón $\left(\varepsilon_{\mathrm{r}}=2,2\right)$, con un diámetro $D=$ $10 \mathrm{~cm}$; un espesor $\mathrm{t}=1 \mathrm{~cm}$, y una distancia focal de $f=40 \mathrm{~cm}$.

Aplicando las ecuaciones (6.3) y (6.4) y considerando la lente discretizada en seis regiones se pueden calcular las permitividades dieléctricas para cada región. En la Tabla 1 se muestran los valores para cada región.

Tabla 6.1: Constante dieléctrica por zonas.

\begin{tabular}{|c|c|c|}
\hline Región & Radio $[\mathbf{c m}]$ & Épsilon relativo $\left(\boldsymbol{\varepsilon}_{\mathbf{r}}\right)$ \\
\hline 1 & 0,00 & 2,2 \\
\hline 2 & 0,91 & 2,17 \\
\hline 3 & 1,81 & 2,08 \\
\hline 4 & 2,73 & 1,93 \\
\hline 5 & 3,64 & 1,74 \\
\hline 6 & 4,55 & 1,5 \\
\hline
\end{tabular}


La Figura 6.10 muestra un diagrama 3D del campo lejano de la antena lente simulada mediante el software de simulación FEKO 7.0 [51]. No fueron simuladas las pérdidas del material dieléctrico.

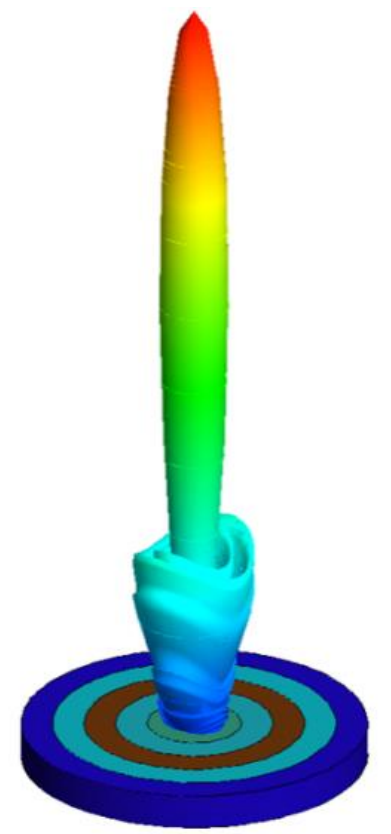

Figura 6.10: Modelo simulado 3D.

Se realizaron simulaciones para la bocina sola, por un lado, y por otro para la antena lente, es decir la bocina junto con la lente.

En la Figura 6.11 se observa el resultado de la ganancia obtenido de las simulaciones, considerando la bocina sola, y el conjunto antena lente (bocina + lente). 


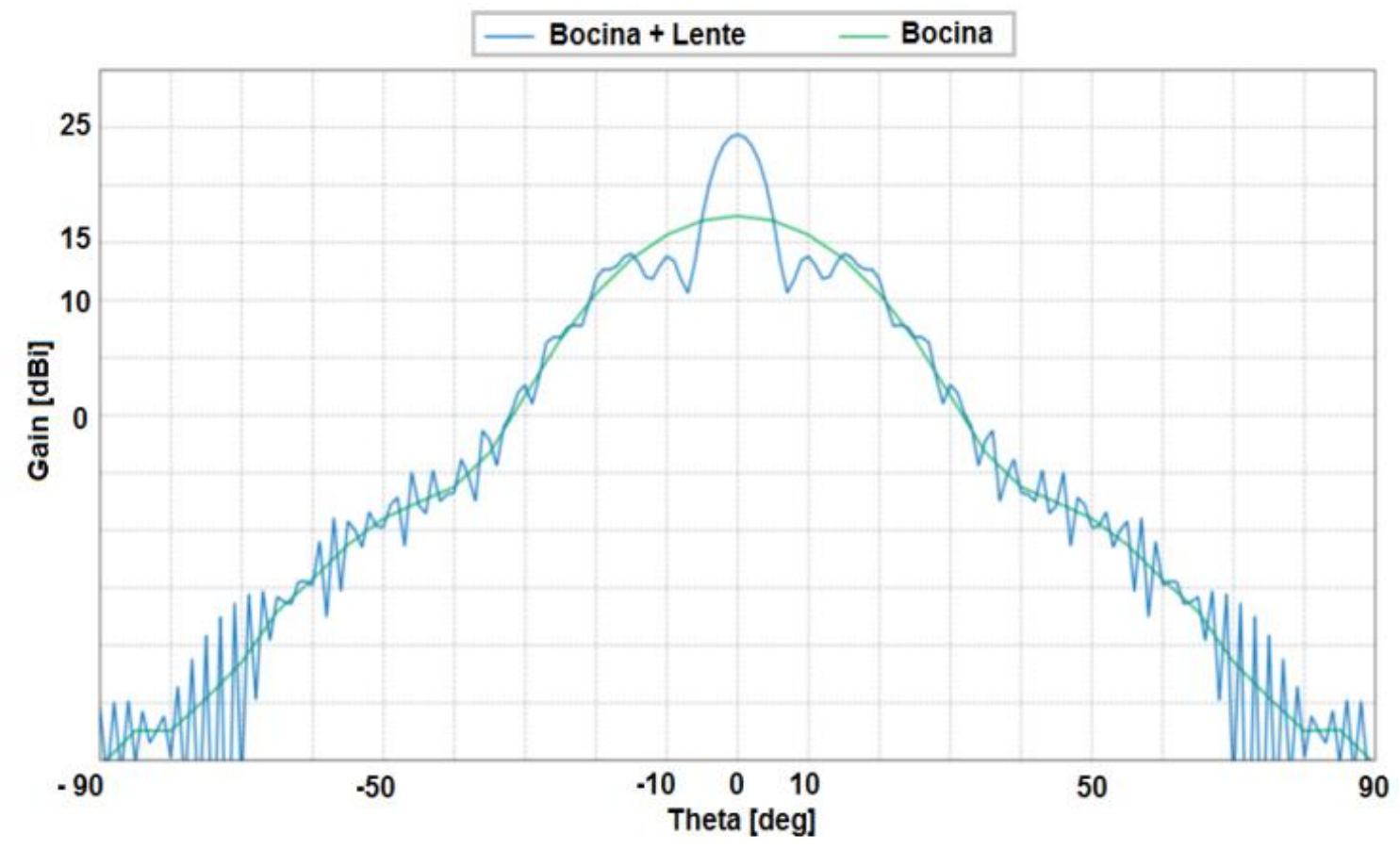

Figura 6.11: Ganancia de la antena lente simulada en $23 \mathrm{GHz}$.

Como se observa el ancho del haz efectivo donde tiene efecto la lente (aumento de la ganancia) es aproximadamente de $+-5^{\circ}$.

El tiempo de simulación fue de aproximadamente 4hs.

\subsubsection{Resultados de antena lente plana dieléctrica}

Por otro lado, se realizaron mediciones a partir del prototipo construido. La lente se fabricó sobre teflón, con los mismos parámetros de diseño de la lente simulada $(D=10$ $\mathrm{cm} ; t=1 \mathrm{~cm}, \mathrm{y} f=40 \mathrm{~cm})$.

La lente se construyó discretizando el sustrato en seis regiones, tal como el diseño simulado (Tabla 6.1), utilizando el método de perforaciones descripto en el inciso anterior.

Debido a las dimensiones de la perforadora disponible en el laboratorio el diámetro de los orificios resultó de $\mathrm{d}=1,5 \mathrm{~mm}$. La distribución de los orificios para cada zona se obtiene aplicando las ecuaciones (6.5) y (6.7).

En la figura 6.12 se observa el prototipo de lente construido. 


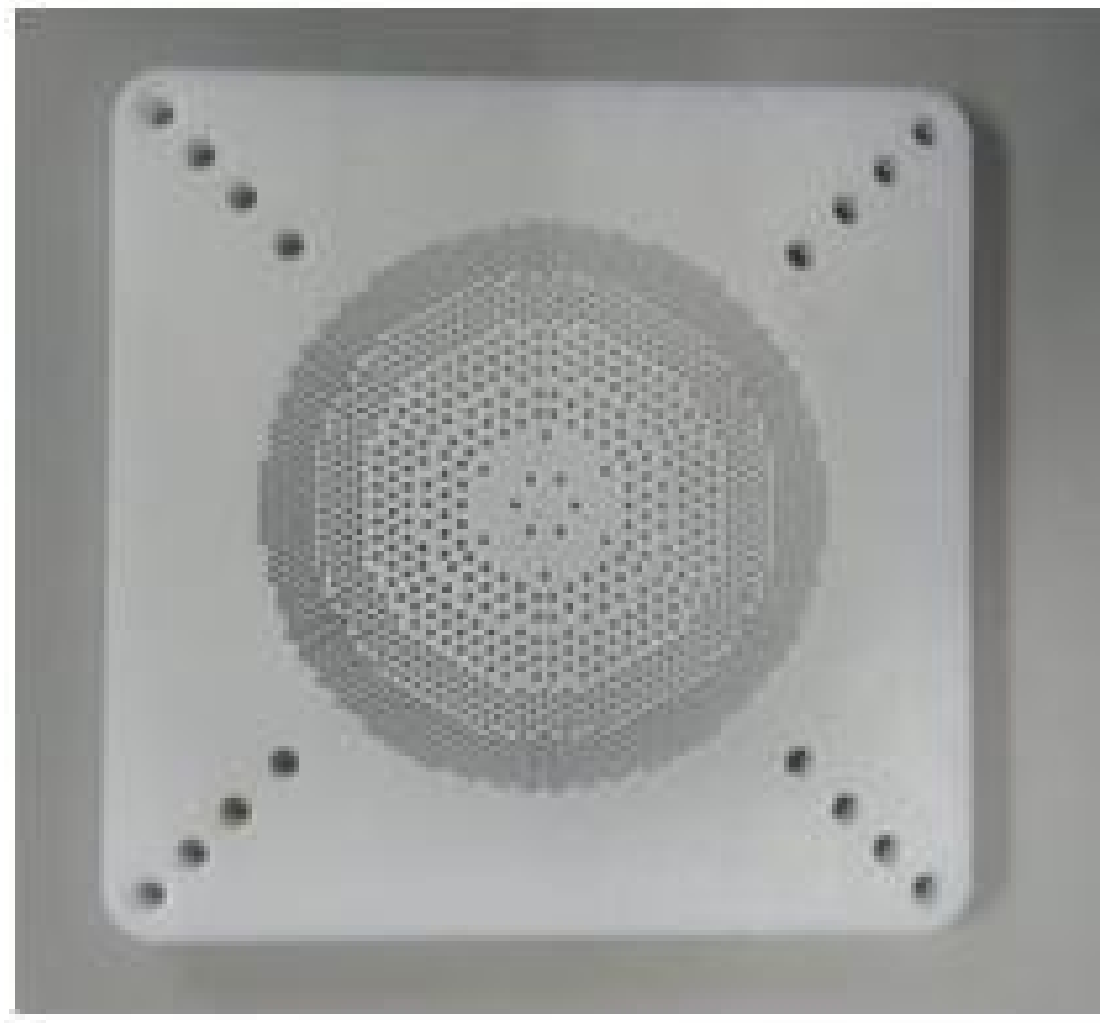

Figura 6.12: Prototipo de Lente plana con orificios.

Se realizaron mediciones con el conjunto antena lente (bocina + lente prototipo) y de la bocina sin la lente con el fin de obtener los diagramas de radiación para comparar con los diagramas simulados. Las mediciones se realizaron para ángulos de $+-5^{\circ}$ para comparar el haz efectivo [90]. Los resultados se muestran en la Figura 6.13.

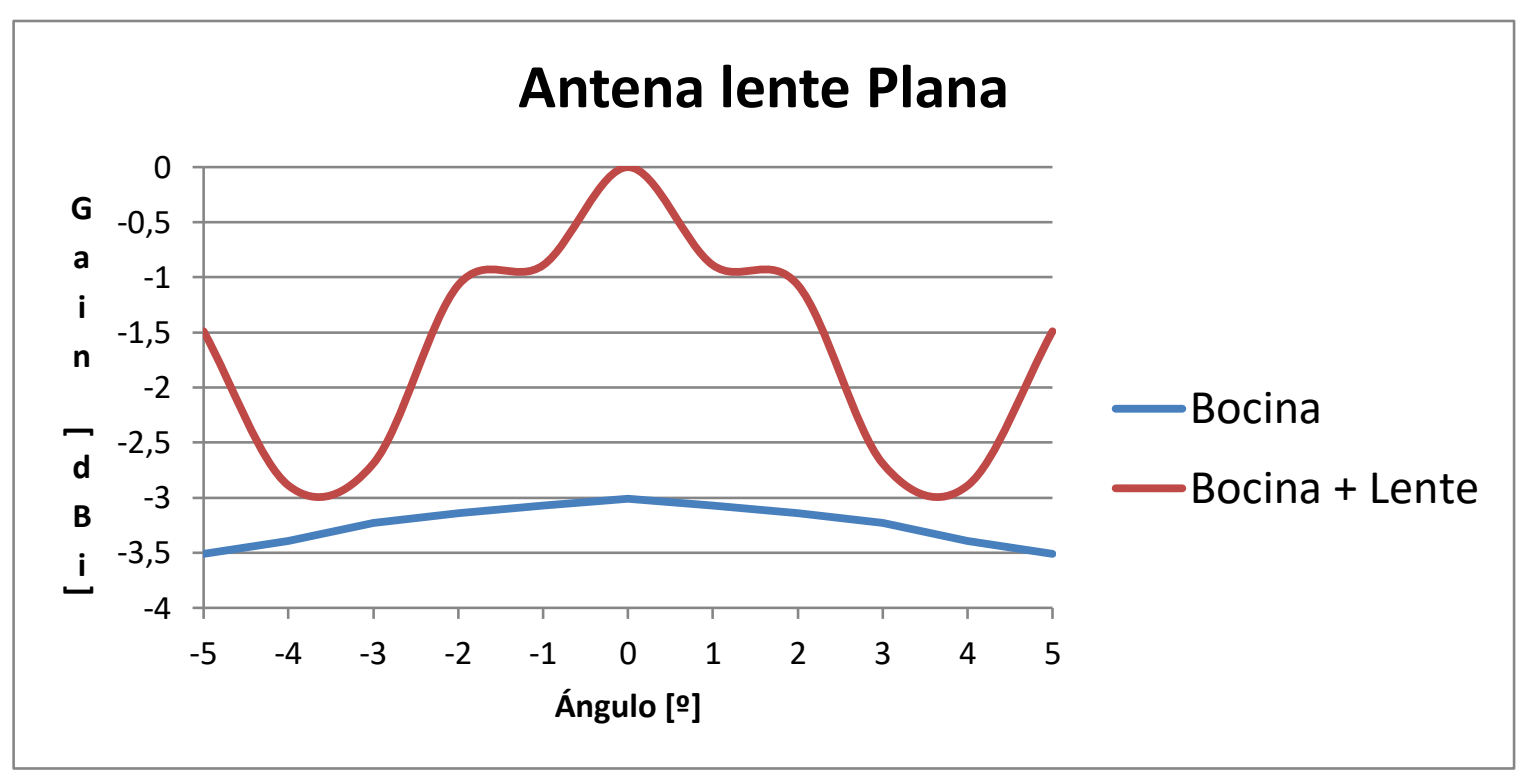

Figura 6.13: Medición de antena lente plana con orificios. 
En la Figura 6.14 se superponen los resultados de las simulaciones y las mediciones, tanto para el conjunto antena lente (bocina + lente prototipo) como para la bocina sin la lente.

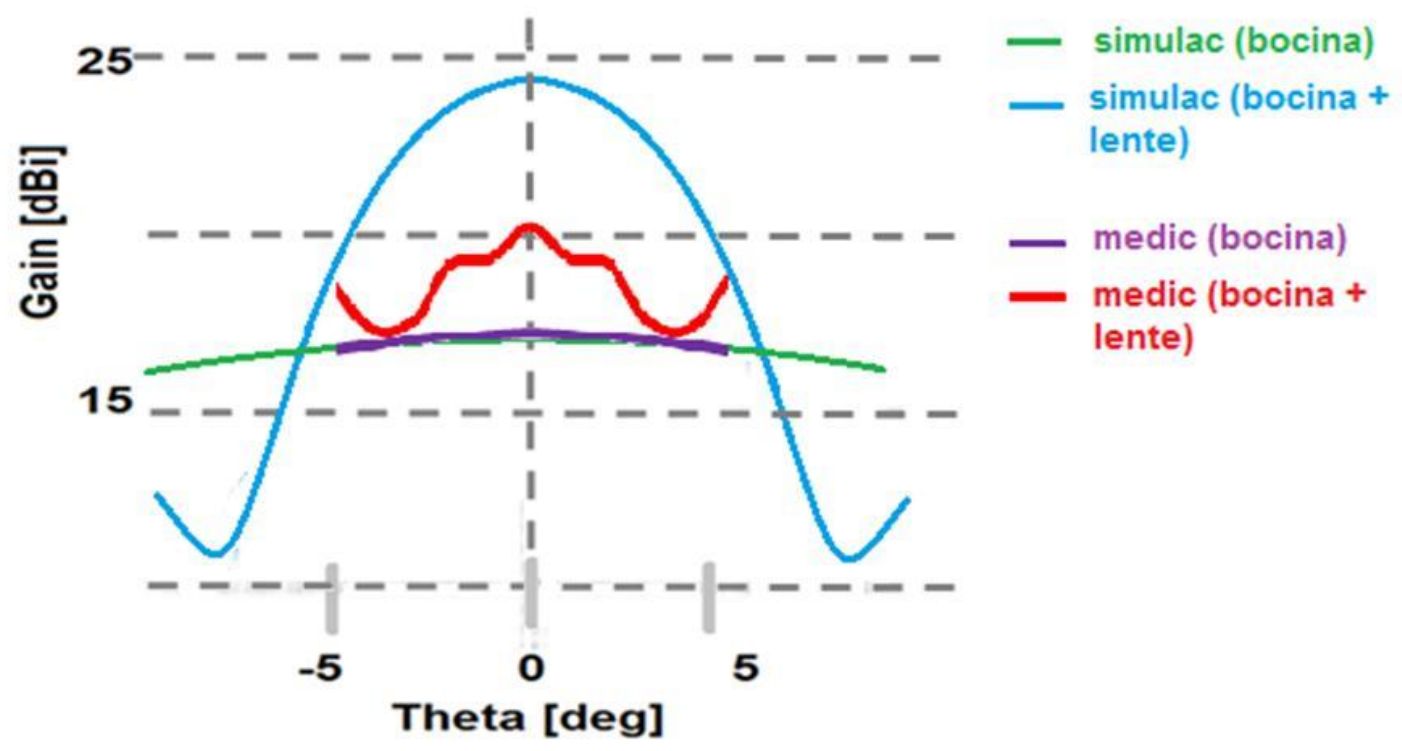

Figura 6.14: Ganancia simulada y medida para bocina y para antena lenta (bocina + prototipo) en $23 \mathrm{GHz}$.

De las simulaciones se deduce que la ganancia de la antena lente es aproximadamente $6 \mathrm{~dB}$ superior a la de la bocina, en su dirección de máxima radiación (Theta $=0 \mathrm{deg}$ ). En el caso de las mediciones la diferencia es algo menor, aproximadamente $3 \mathrm{~dB}$. Dicha diferencia se debe a que la simulación no tiene en cuenta las perdidas por transmisión ni los factores de alineación de la lente. Asimismo, el tamaño de los agujeros no es completamente despreciable con respecto a la longitud onda.

La fabricación de la antena se realizó mediante una máquina de control numérico (CNC) en Instituto de Telecomunicaciones y Aplicaciones Multimedias (iTEAM) de la Universidad Politécnica de Valencia - España. 


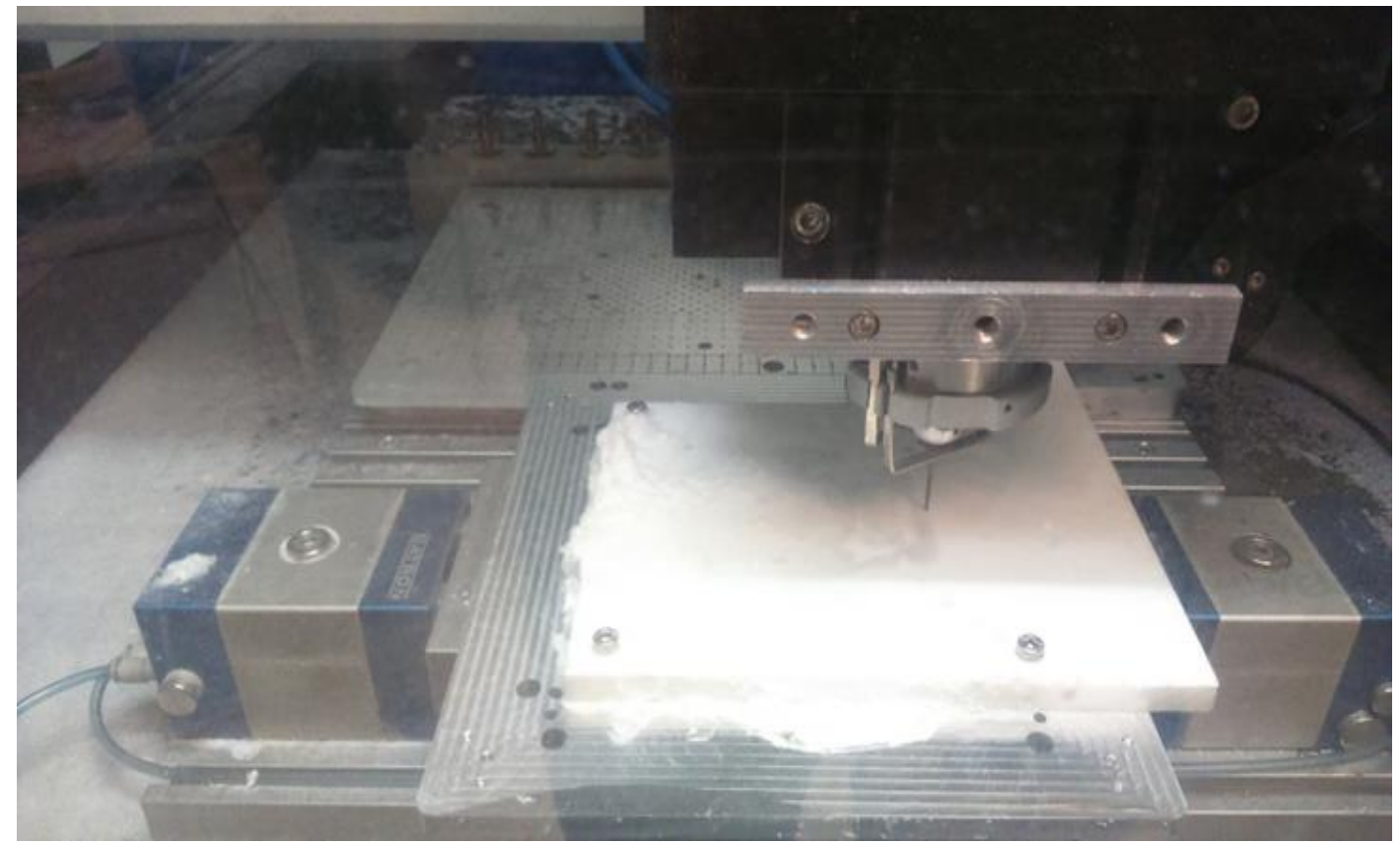

Figura 6.15: Fabricación de la antena lente plana dieléctrica.

Las mediciones de la antena se realizaron en la cámara anecoica el ITEAM

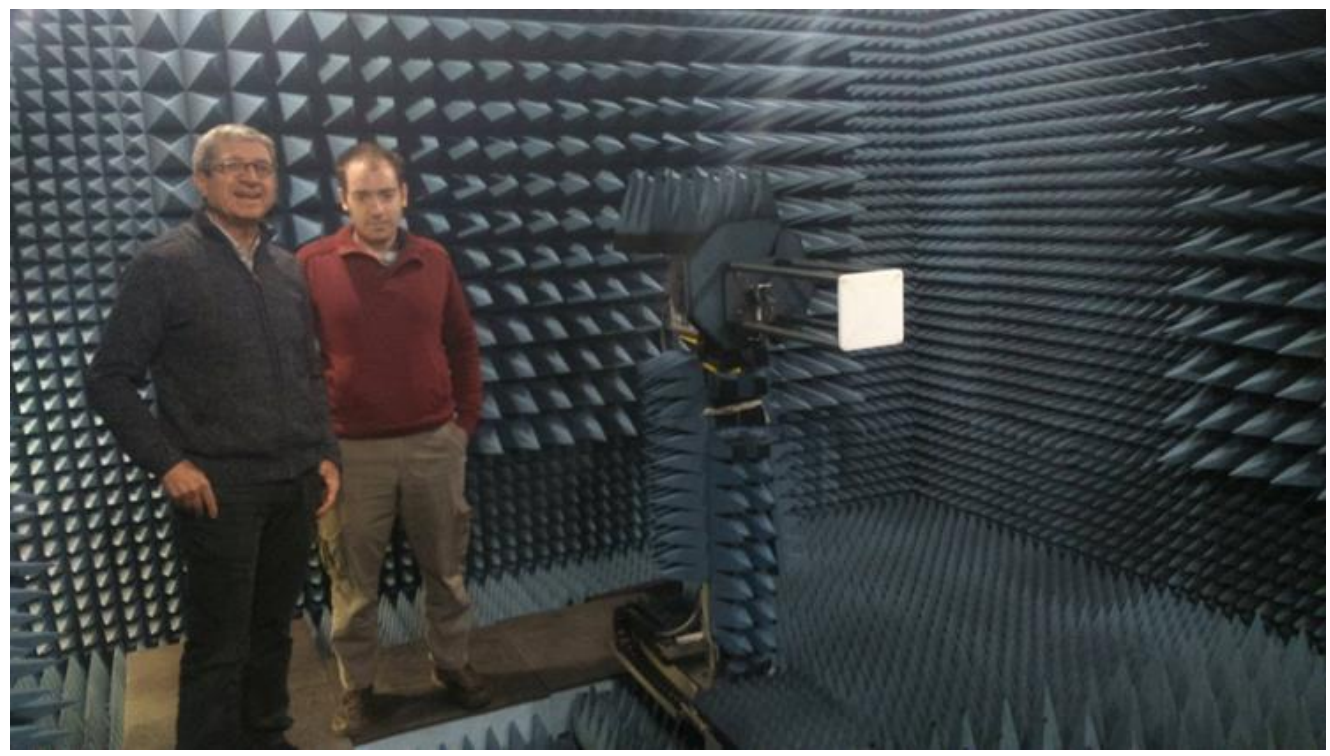

Figura 6.16: Medición de la antena plana dieléctrica.

\subsection{Antena lente de zonas de Fresnel}

La lente de zonas de Fresnel (FZP) se obtiene de colocar anillos metálicos concéntricos sobre una superficie dieléctrica a fin de lograr los desfasajes radiales deseados (Figura 6.17). La lente FZP actúa como una rejilla de difracción de elementos anulares [91]. 


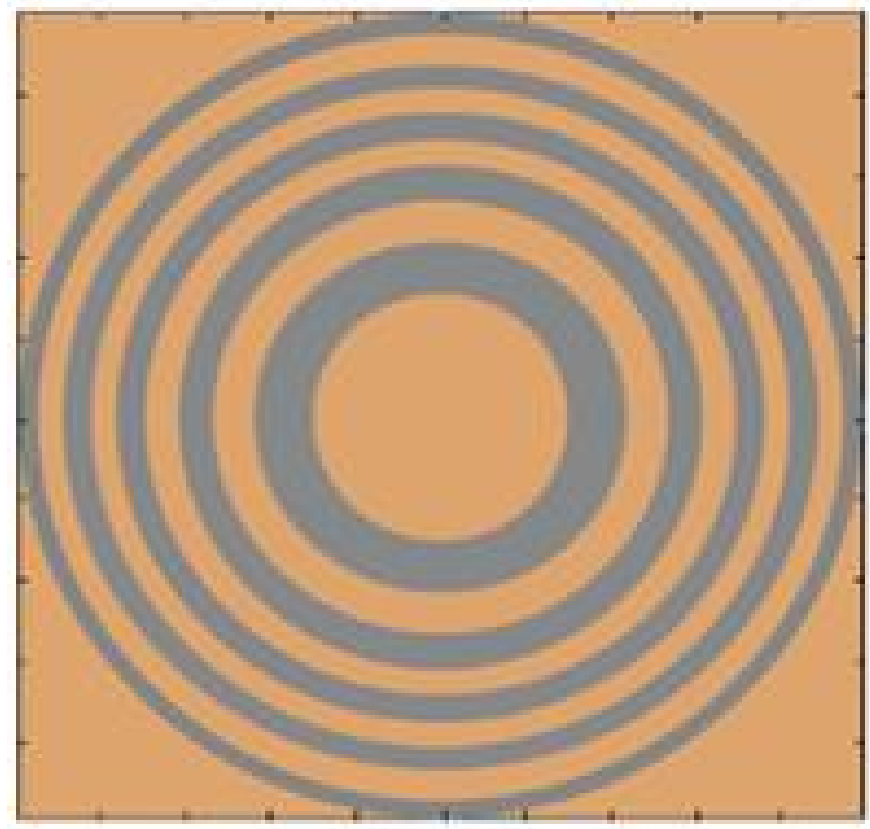

Figura 6.17: Lente de zonas de Fresnel (FZP).

Las lentes de Fresnel son más económicas y fáciles de fabricar debido a su configuración y de un peso total mucho menor comparadas con otros tipos de antenas lentes.

En general, una lente FZP centrada en el origen de un sistema de coordenadas Cartesiano transforma un frente de onda esférica incidente con radio $z=-F_{s}$, en múltiples ondas esféricas enfocadas en el eje axial $\mathrm{z}$ con múltiples focos con distancia focal $z=$ $+F_{n}$, con $n=1,3,5, \ldots$ impares. La característica inusual de la lente FZP es su propiedad multifocal, la cual se debe a su naturaleza difractiva, y ésta es la mayor diferencia con las lentes típicas difractivas.

\subsubsection{Diseño de lente de zonas de Fresnel}

La lente responde a la siguiente ecuación [91]:

$$
b(m)=\sqrt{m \lambda F+\left(\frac{m \lambda}{2}\right)^{2}}
$$

donde $b(m)$ es el radio de las zonas de Fresnel, $\lambda$ es la longitud de onda y $m=1$, $2,3, \ldots .$. , es el número de zona y $F$ es la distancia focal. La Figura 6.18 muestra un diagrama esquemático del diseño de la lente. 


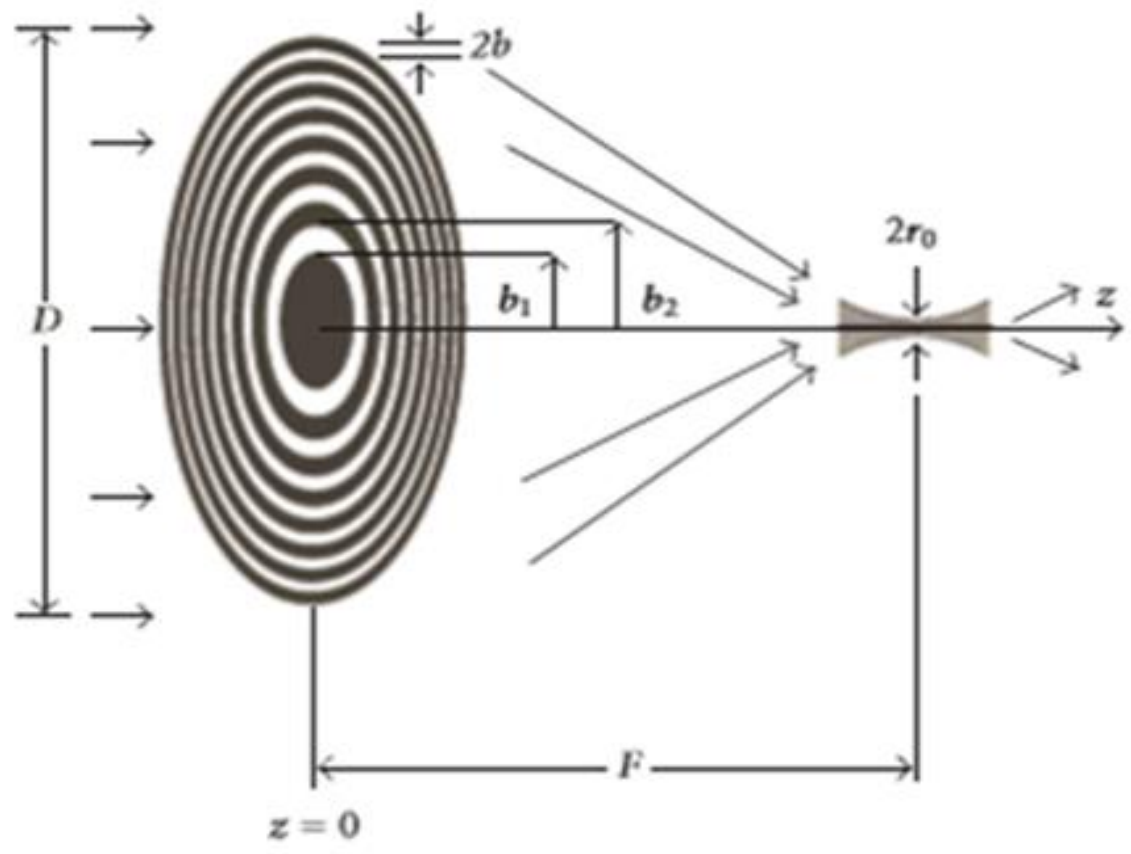

Figura 6.18: Esquema de lente de zonas de Fresnel.

\subsubsection{Simulaciones de antena lente de zonas de Fresnel}

Utilizando la ecuación 6.9 se diseña y simula una antena para $23,8 \mathrm{GHz}$, iluminada con una bocina cónica, con 5 anillos (10 radios), con una relación aproximada de $f / D=1$. Las simulaciones se realizaron mediante el software Feko.

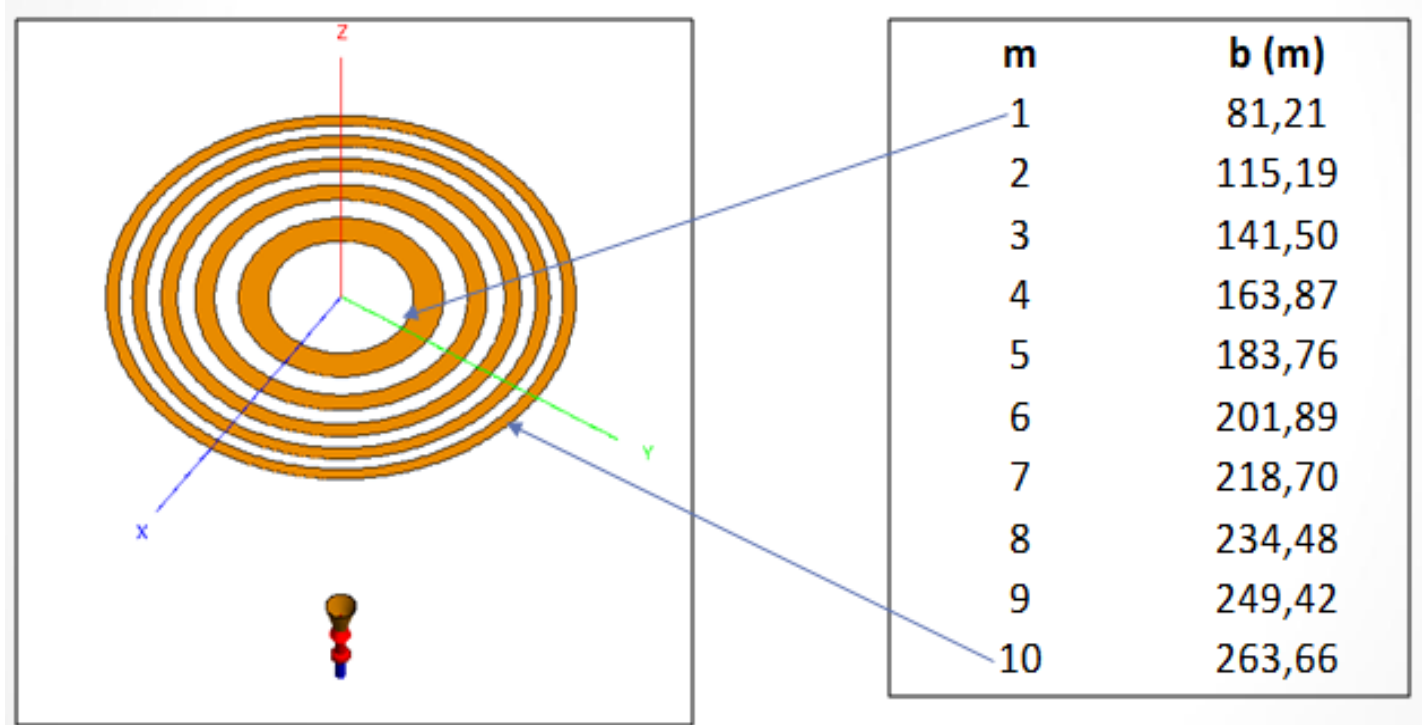

Figura 6.19: Diseño de lente de zonas de Fresnel. 
El diámetro de la lente es de aproximadamente $D=527 \mathrm{~mm}$

Los resultados de la simulación de la bocina sola y la bocina con la lente se muestran en la Figura 6.20 a) y b) respectivamente.

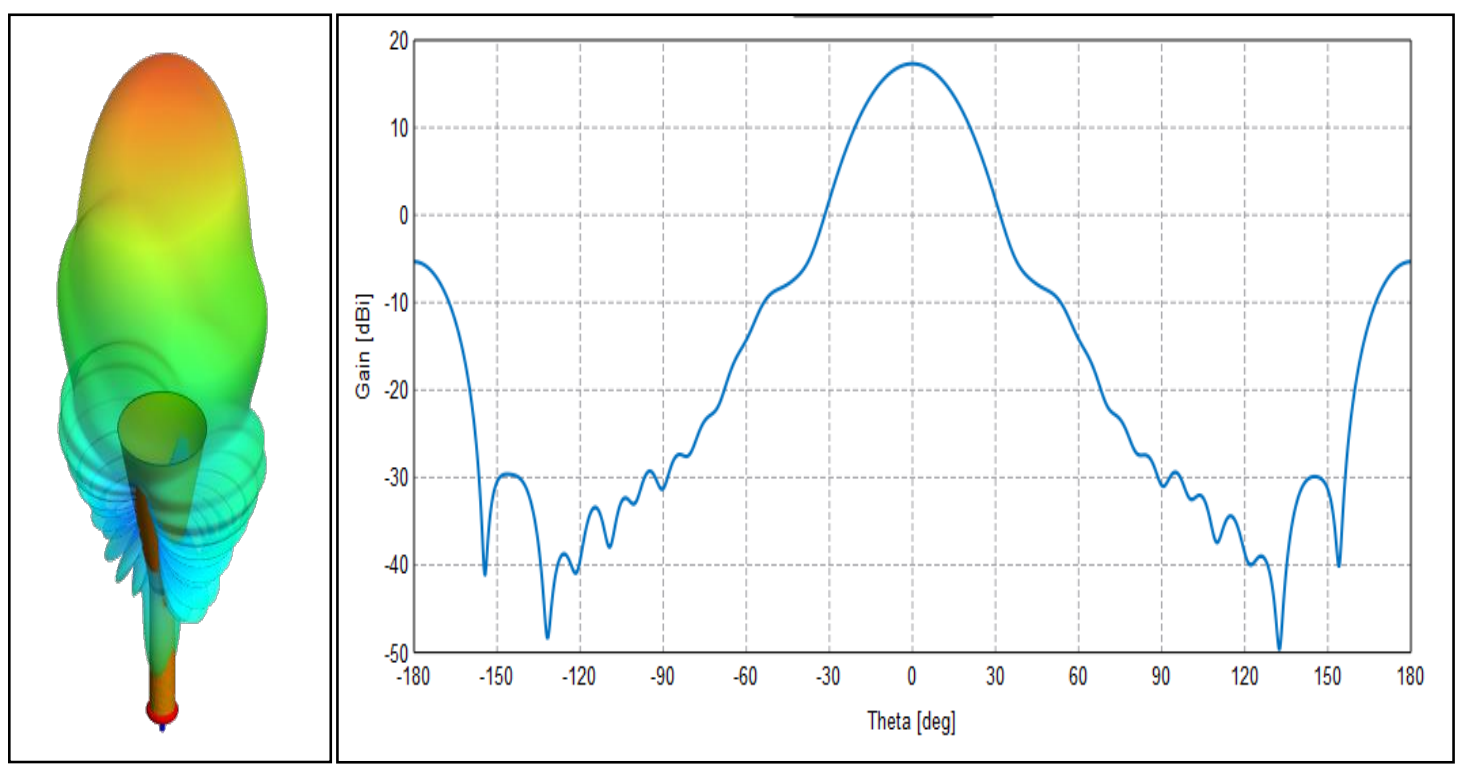

a)
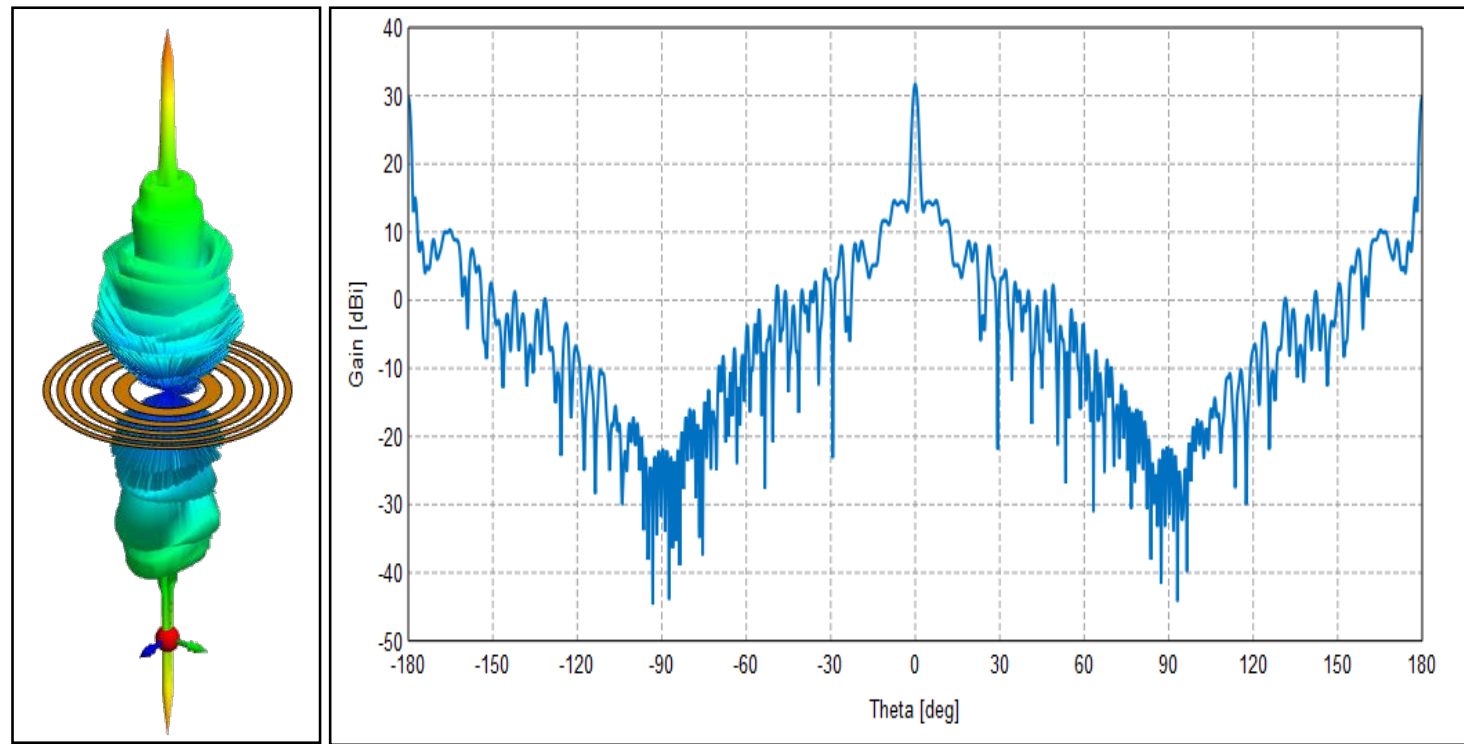

b)

Figura 6.20: Diagrama de radiación simulado de a) la bocina sola y b) la bocina con la lente de zonas de Fresnel.

Con el fin de comparar el efecto de la lente en el alimentador se muestra en la Figura 6.21 los diagramas de radiación superpuestos de la bocina sola y la bocina con la lente. 


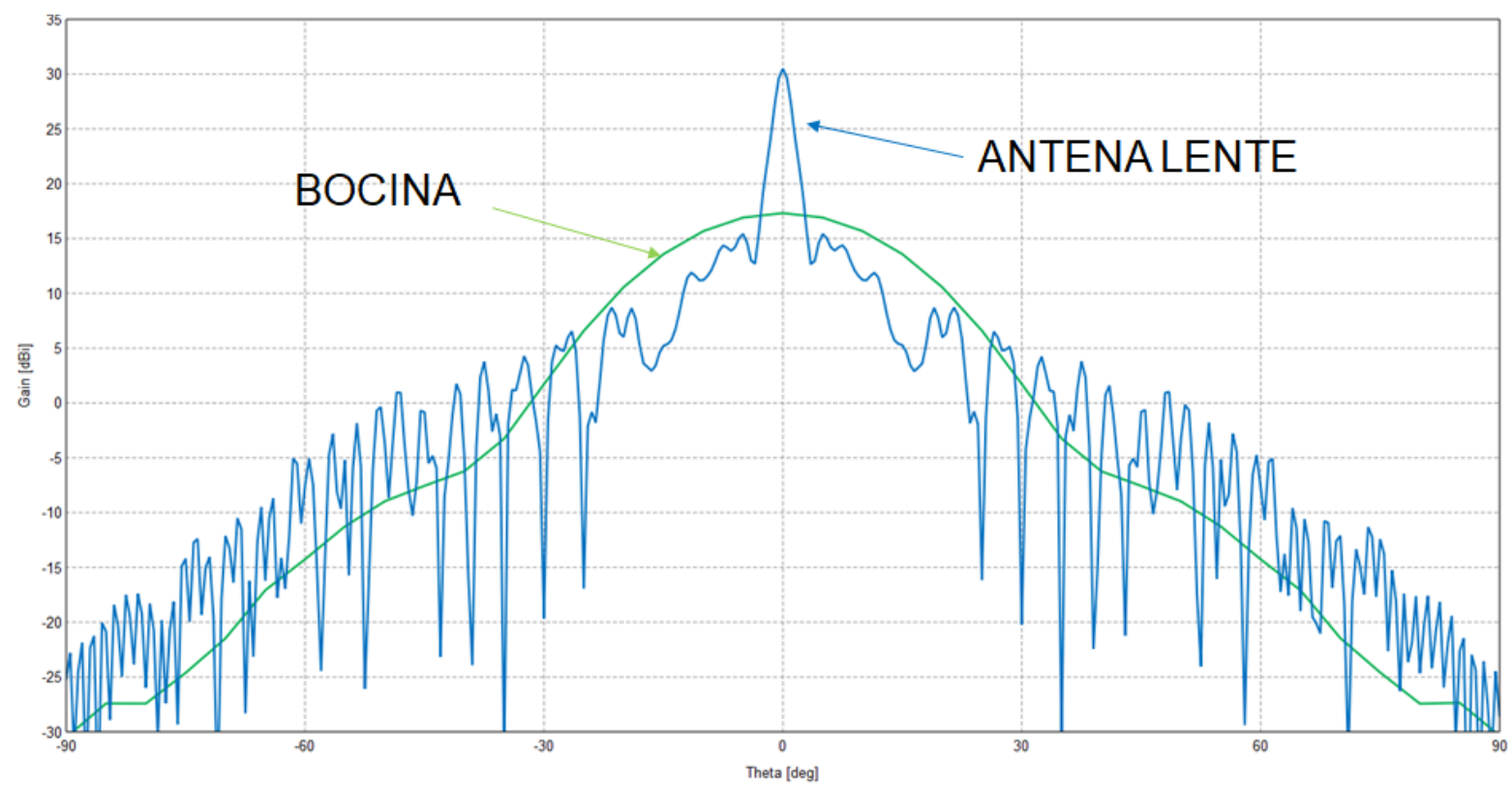

Figura 6.21: Diagrama de radiación de la bocina sola y la antena lente.

Es interesante observar el efecto del desplazamiento del alimentador en uno de sus ejes. La Figura 6.22 muestra el resultado del campo lejano de la antena lente para desplazamientos del alimentador.

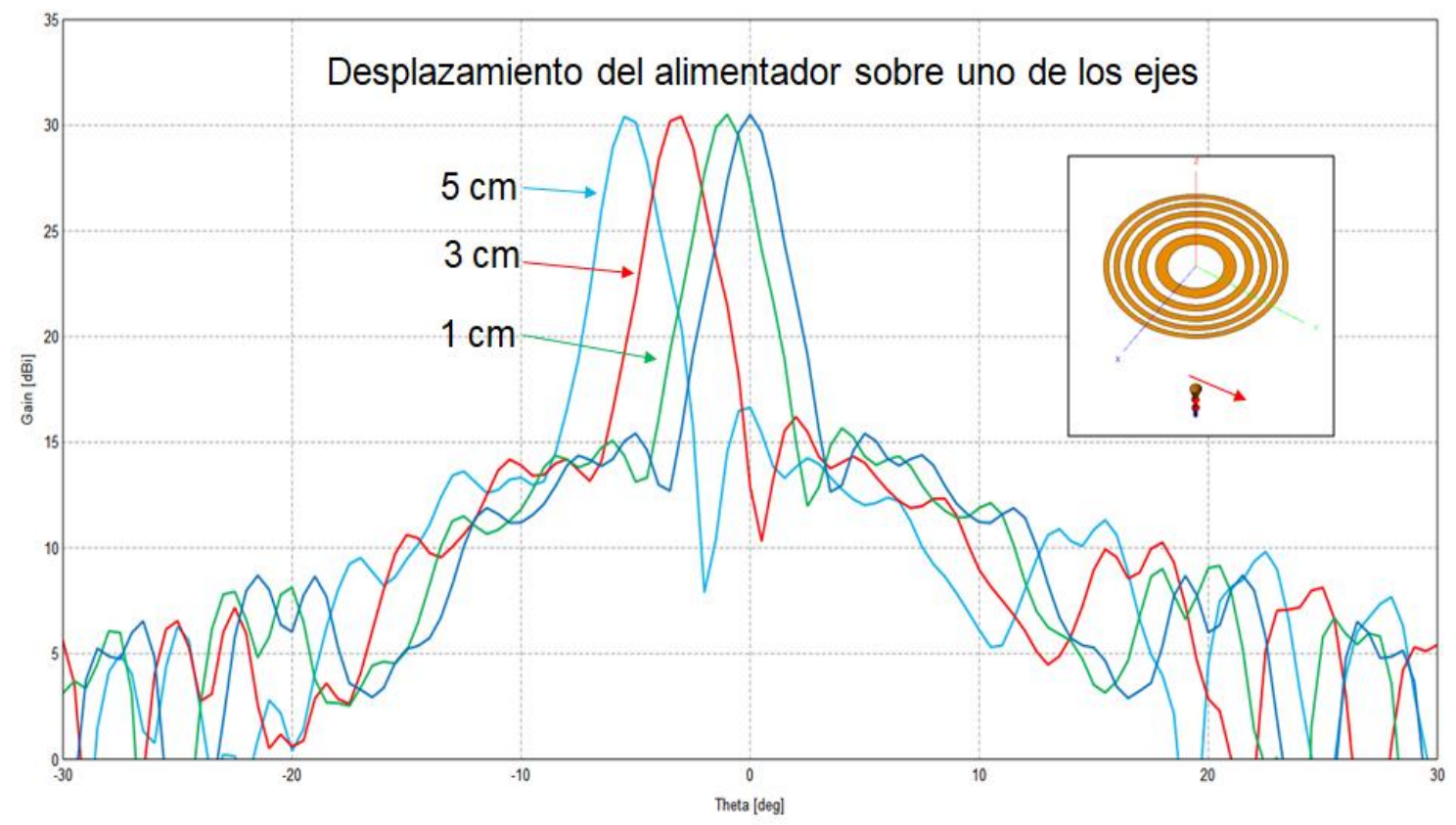

Total Gain (Frequency $=23.8 \mathrm{GHz} ;$ Phi $=0$ deg $)$

Figura 6.22: Diagrama de radiación de la antena lente para diferentes posiciones del alimentador.

Existen numerosas alternativas para la construcción de la lente de Fresnel. Algunos ejemplos se observan en la Figura 6.23, donde a) es la lente original simulada previamente, b) es una lente formada por círculos de diámetro igual al ancho de las zonas 
de Fresnel, y c) y d) son lentes cuya primera zona está formada por aros finos y gruesos respectivamente.

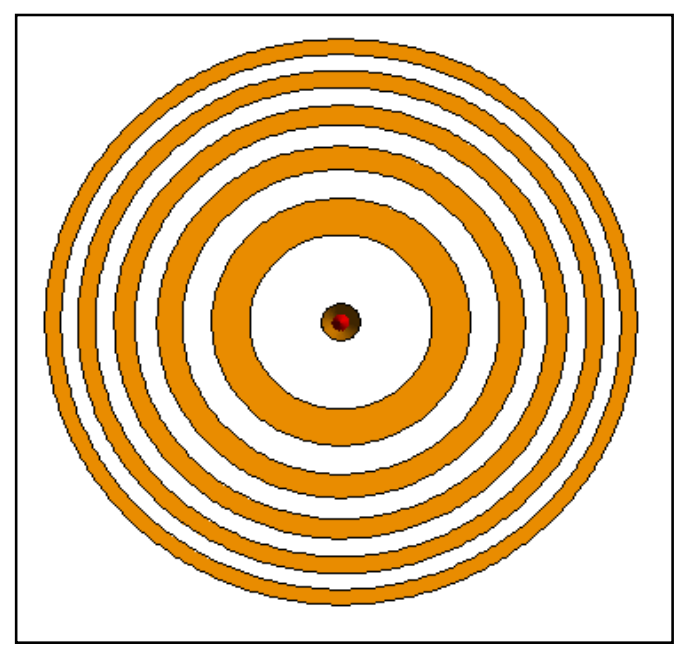

a)

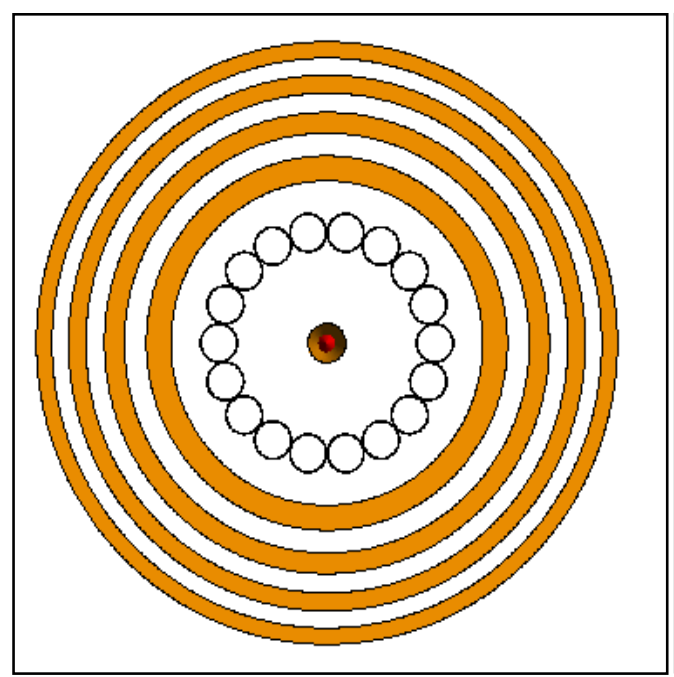

c)

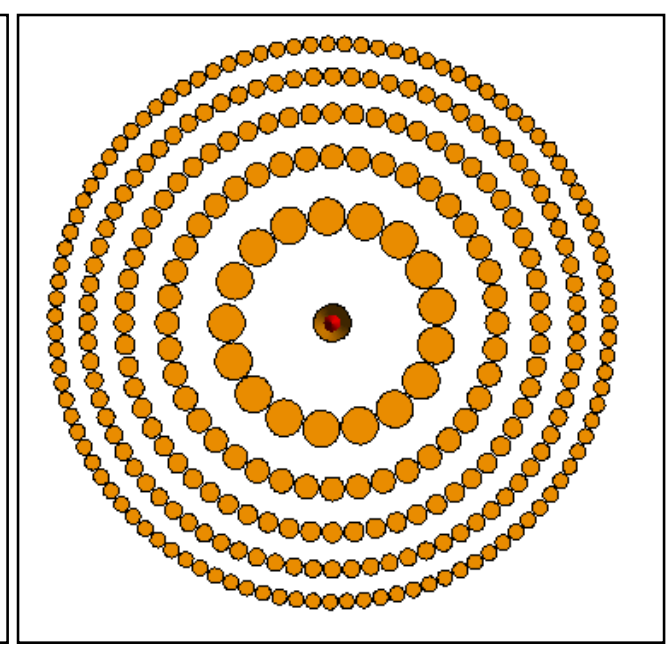

b)

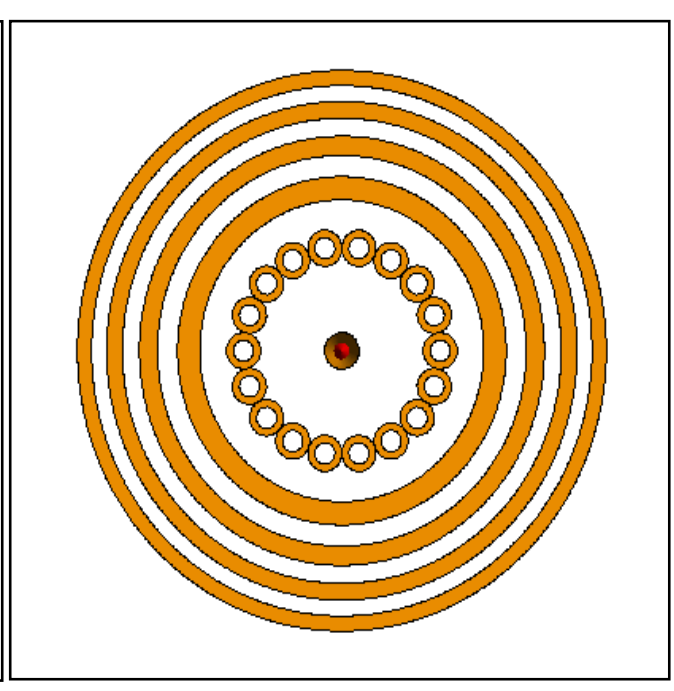

d)

Figura 6.23: Diferentes diseños de la lente de zonas de Fresnel.

La Figura 6.24 muestra el resultado de las simulaciones de los diferentes diseños de lentes realizadas mediante el software Feko. 


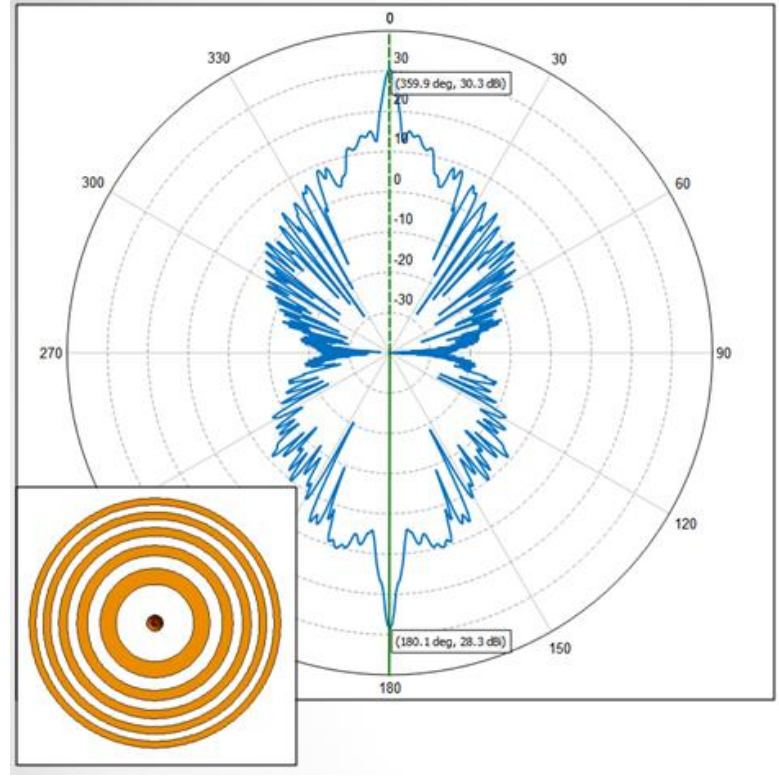

a)

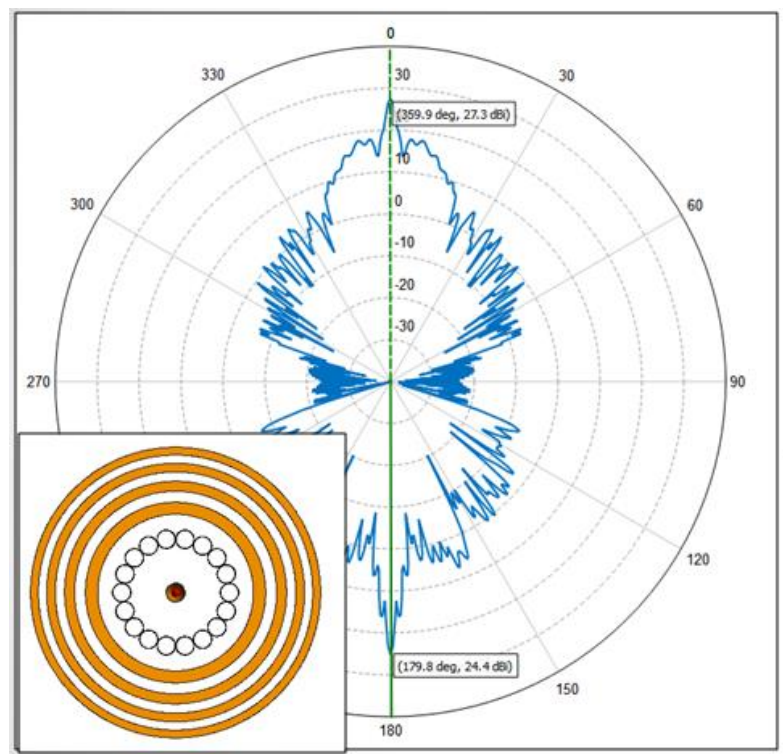

c)

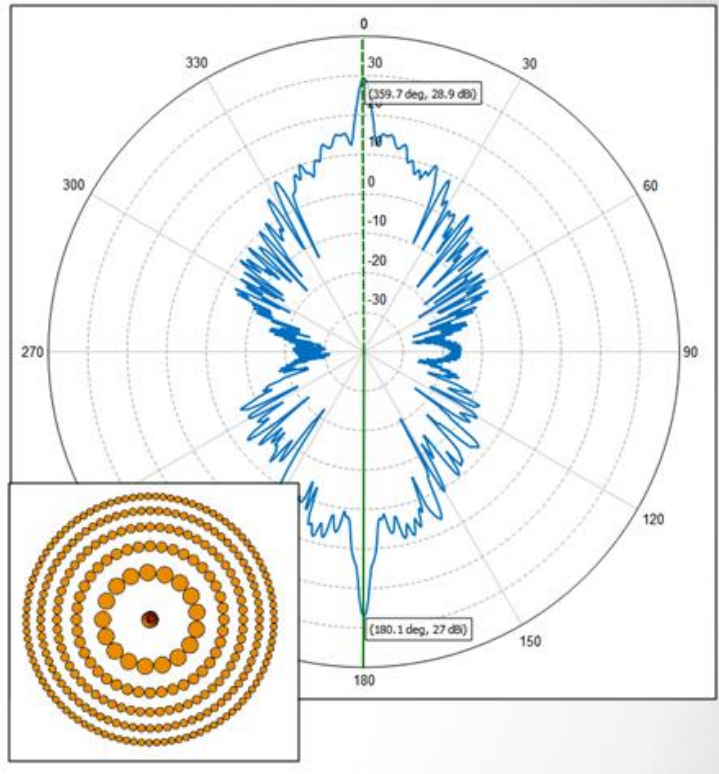

b)

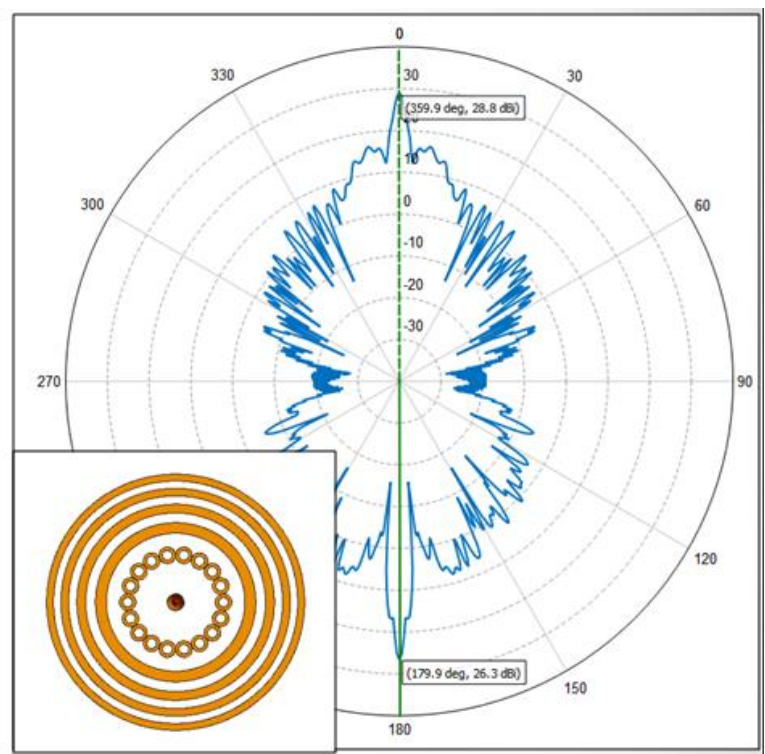

d)

Figura 6.24: Diagrama de radiación simulado de los diferentes diseños de la lente de zonas de Fresnel.

Del diagrama de radiación simulada de los diferentes diseños de la lente de zonas de Fresnel, se observa que, con círculos de diámetro igual al ancho de las zonas de Fresnel, tal como se observa en las figuras 6.24 a) y b), la lente no mejora ni la ganancia y ni la relación front to back comparada con la lente original.

Con aros, tal como se observa en las figuras $6.24 \mathrm{c}$ ) y d), igual del ancho de las zonas de Fresnel, no mejora ni la ganancia y ni la relación front to back con respecto a la lente original. 


\subsubsection{Resultados de antena lente de zonas de Fresnel}

Con el fin de comparar modelos simulados con prototipos construidos se simuló y construyó una antena binaria de zonas de Fresnel en la frecuencia de $23 \mathrm{GHz}$.

La antena lente se construyó montando anillos metálicos sobre una placa de material dieléctrico (Rohacel HF foam) que posee una constante dieléctrica muy próxima a la unidad y con una tangente de perdida muy baja. La distancia f/D es de 0,46, con 3 anillos concéntricos y un diámetro de la lente de $172 \mathrm{~mm}$ (Figura 6.25), siendo su peso total de 100 gramos.

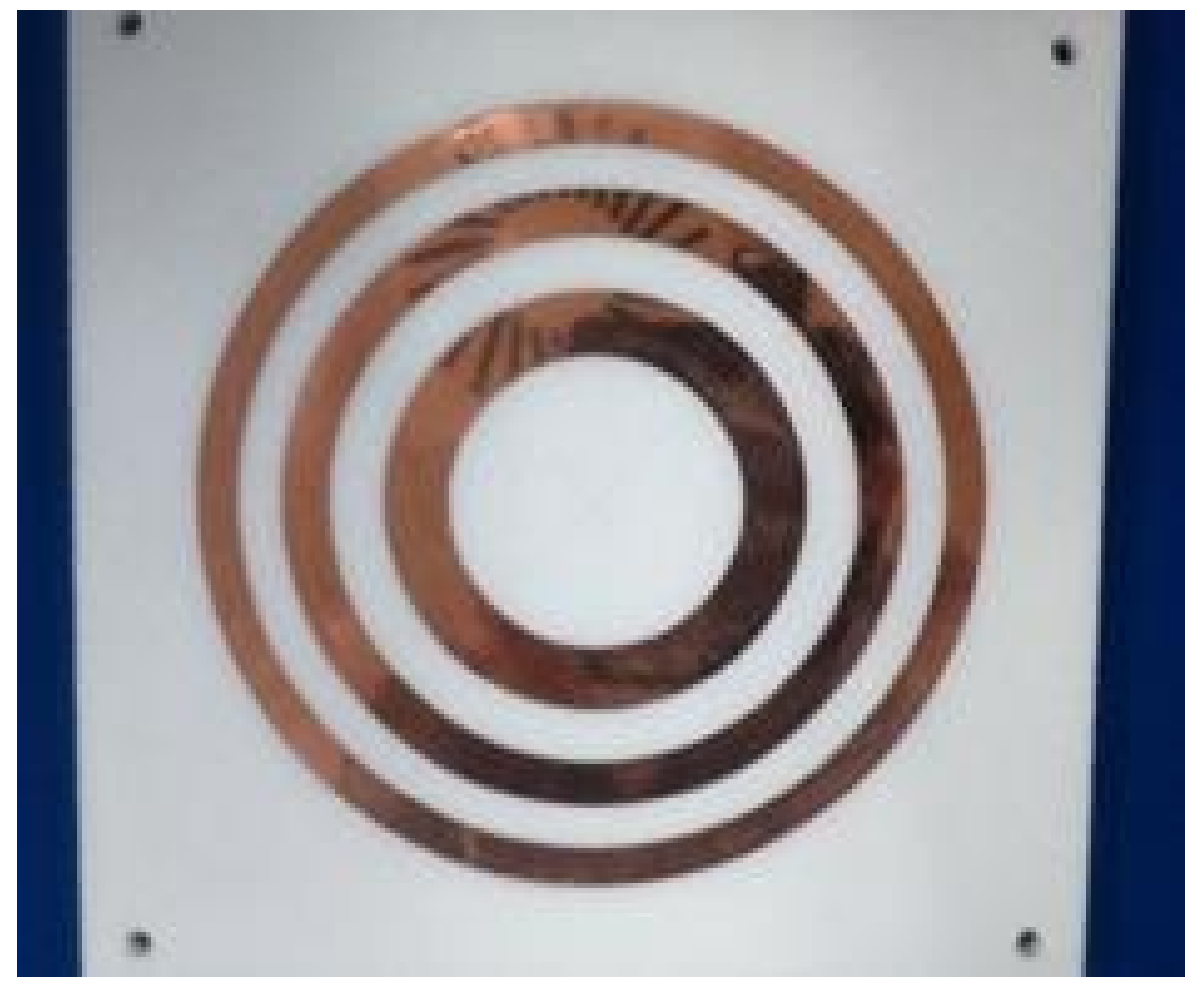

Figura 6.25: Antena lente de zonas de Fresnel Construida.

Esta antena ofrece una interesante alternativa como antena de haces múltiples, logrando mover el haz principal solo con un simple desplazamiento en el plano xy del alimentador principal (Figura 6.26). 


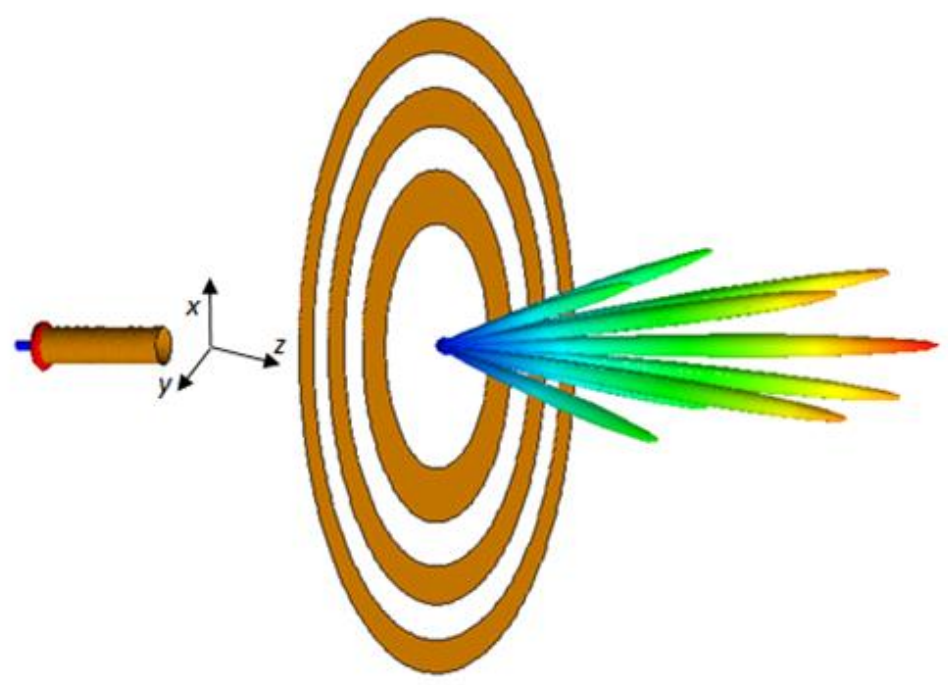

Figura 6.26: Simulación 3D mediante el software de simulación Feko de la antena lente de zonas de Fresnel Construida.

En la Figura 6.27 se observa el resultado obtenido de la simulación de la antena lente diseñada mediante el software de simulación Feko para diferentes posiciones discretas del elemento radiante a lo largo de la dirección del eje $\mathrm{x}$.

Los resultados obtenidos de la simulación muestran la posibilidad de mover el haz principal en ángulos mayores de $30^{\circ}$ con solo un desplazamiento de $60 \mathrm{~mm}$ sobre el eje $\mathrm{x}$ para las características de esta lente. Valores mayores a los $20 \mathrm{~dB}$ de ganancia se obtienen en los ángulos centrales, mientras decae en $3 \mathrm{~dB}$ cuando el haz se desvía en los $30^{\circ}$.

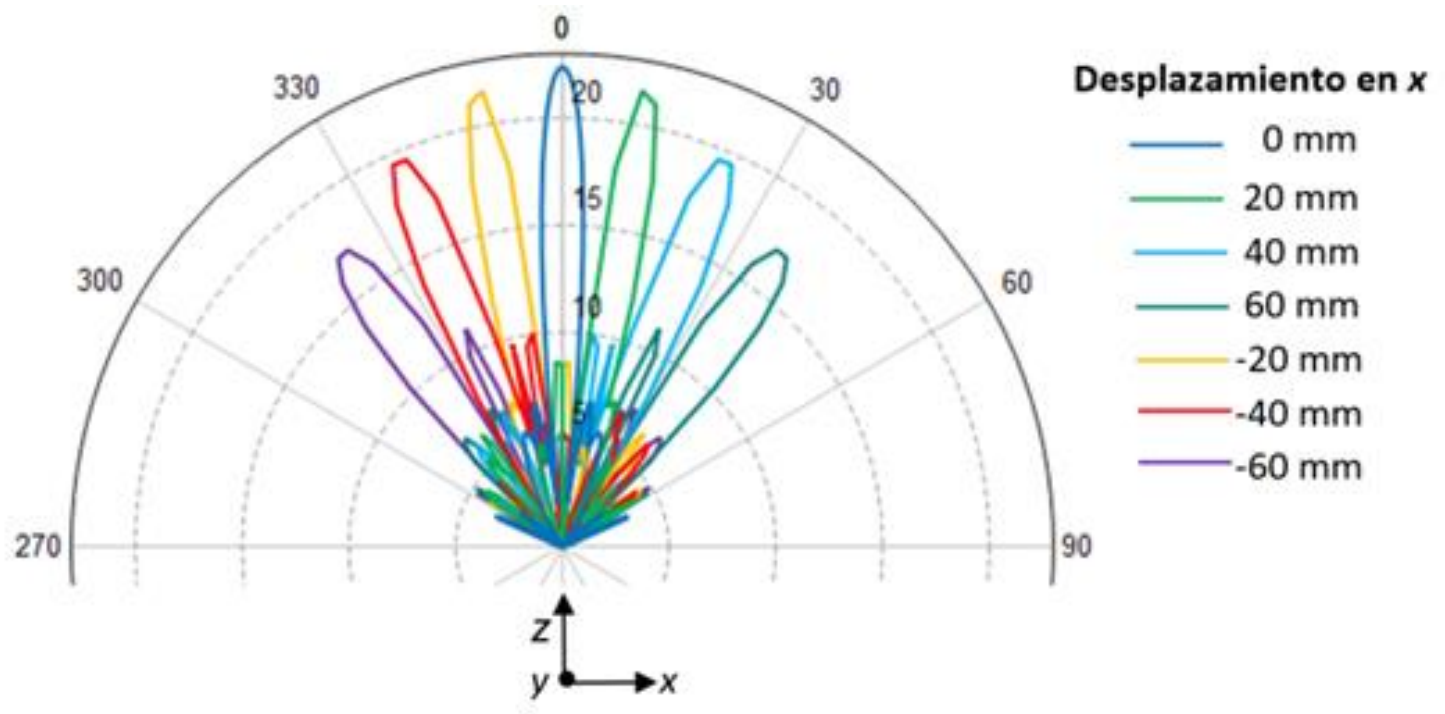

Figura 6.27: Diagrama de radiación simulado para diferentes desplazamientos del alimentador.

La Figura 6.28 el diagrama cartesiano de campo lejano de la antena lente simulada. 


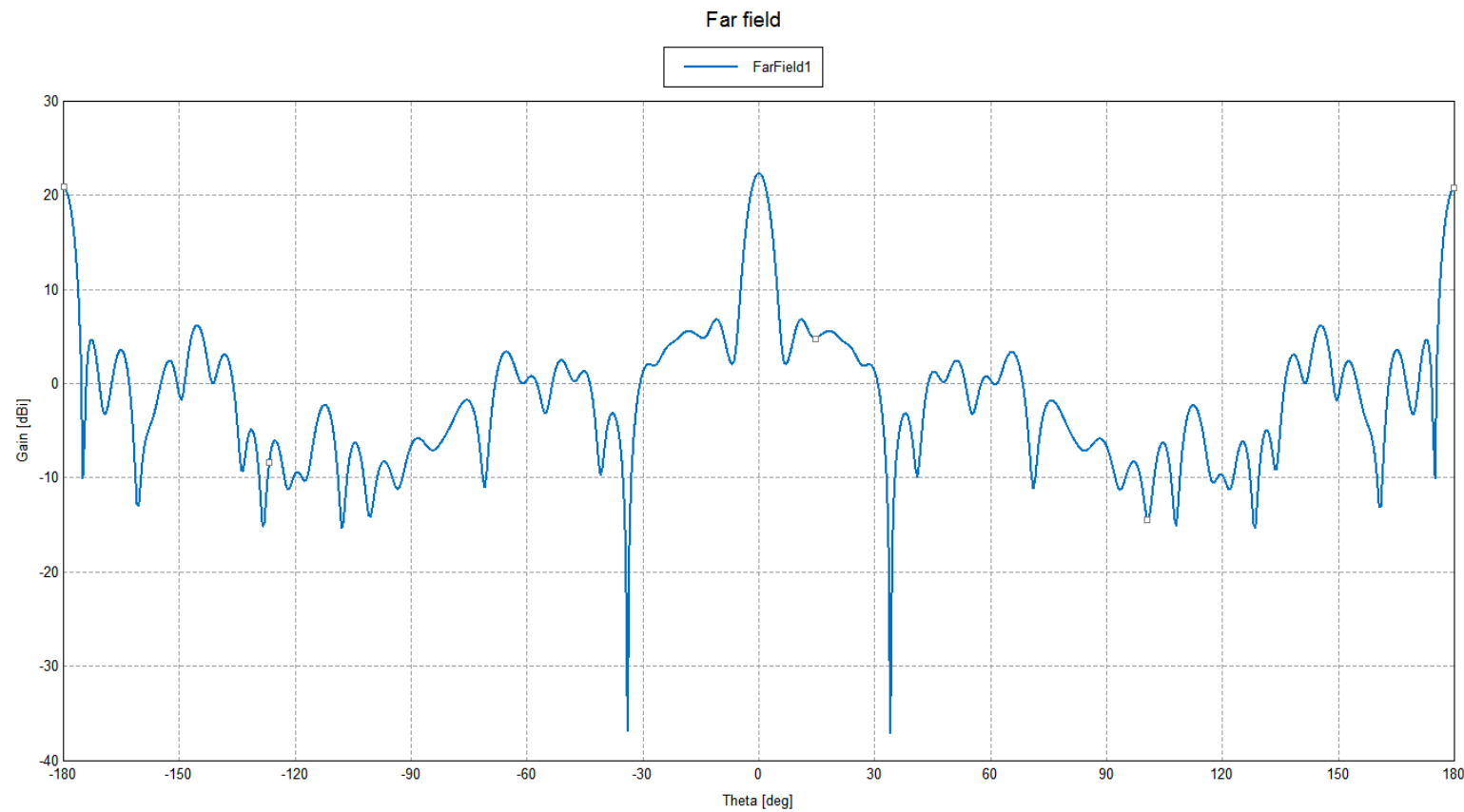

Figura 6.28: Diagrama cartesiano de campo lejano de la antena lente simulada.

Se realizaron medidas del modelo diseñado iluminando la antena lente con una boca de guía de onda, obteniéndose los resultados en 3D que se muestran en la Figura 6.29 .

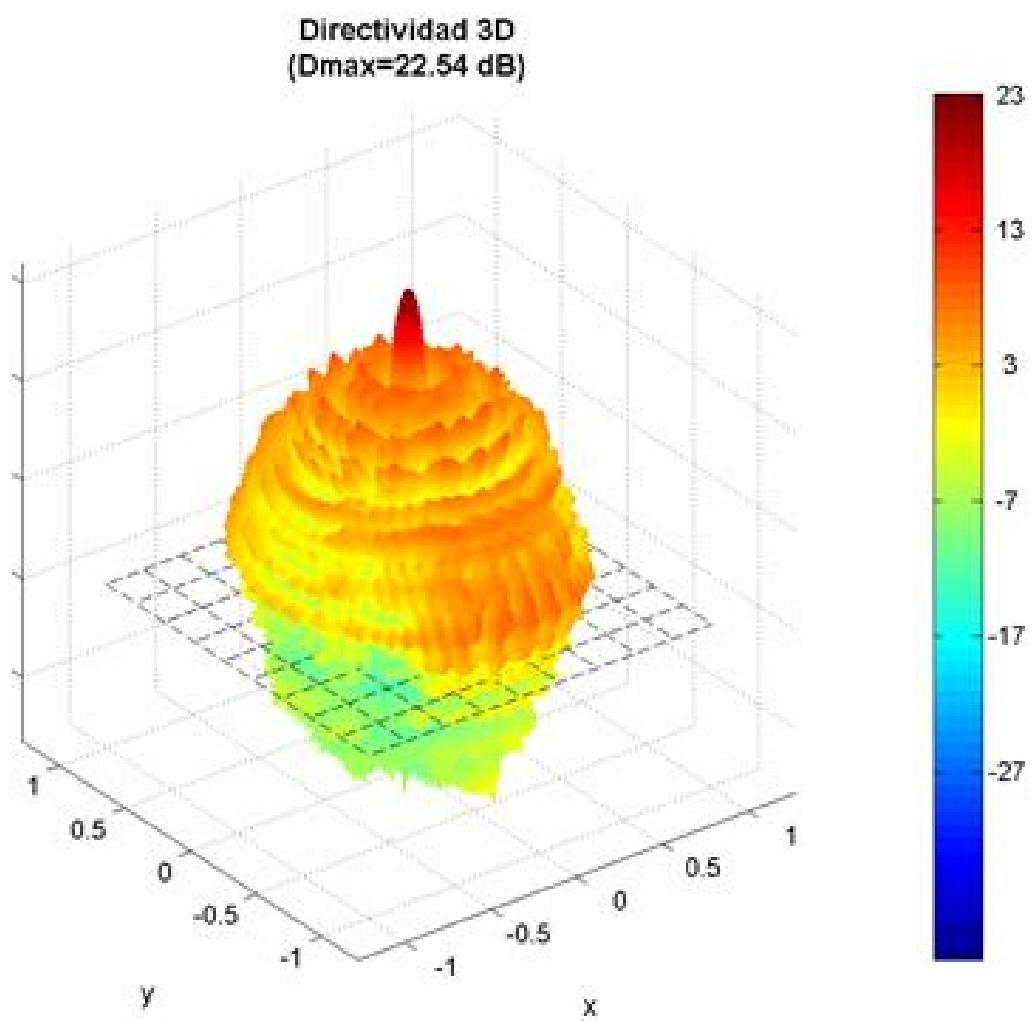

Figura 6.29: Diagrama de radiación medido del prototipo de la antena de zonas de Fresnel. 
Los valores obtenidos de las medidas muestran que la antena lente FZP posee una ganancia máxima de $22,54 \mathrm{~dB}$ y un ancho de haz de $4^{\circ}$ a la frecuencia central de diseño $(23 \mathrm{GHz})[92]$. 


\section{CAPÍTULO 7}

\section{Conclusiones}

\subsection{Conclusiones}

El objetivo principal de esta Tesis ha sido el análisis de diferentes antenas lente para aplicaciones de microondas mediante métodos de simulación electromagnética.

A partir del estado del arte, se investigaron los diferentes tipos de antenas lentes actuales y sus principales características. El desarrollo del electromagnetismo computacional permite realizar simulaciones que contribuyen a profundizar el estudio de estos dispositivos. Particularmente en las aplicaciones que utilizan la banda de microondas se advirtió la existencia de importantes limitaciones en los actuales softwares de simulación electromagnética. Consecuentemente un estudio de los métodos numéricos aplicados por el electromagnetismo computacional (Capítulo 2) resultó fundamental. En esta banda del espectro la aplicación de métodos full-wave provee información más precisa que los métodos asintóticos, pero se requieren amplios recursos computacionales. Sin embargo, el desarrollo a partir de un algoritmo matemático de un novedoso código de simulación electromagnética que aplica técnicas full-wave nos permitió realizar simulaciones en tiempos razonables y con una PC de uso personal. Los resultados obtenidos en el estudio de las antenas lente dieléctricas, aplicando este código, permitieron obtener diversos resultados novedosos. A partir de ello se estableció un método de diseño basado en dicha técnica. El método de diseño dio origen al título de la presente Tesis y se utilizó la palabra óptimo debido a la precisión de la técnica de simulación empleada.

Asimismo, se observa, del estado del arte, que las últimas tendencias en antenas lentes en microondas se basan en diseños de lentes planas o superficies correctoras de fase. Es por ello que se diseñaron y construyeron 2 prototipos de antenas lentes planas; la antena plana dieléctrica y la antena de zonas de Fresnel. Fue posible medir las características de ambas antenas y compararlas con las simulaciones.

Los resultados de la aplicación del código de simulación desarrollado para la presente Tesis, pueden ser extensibles al estudio de múltiples problemáticas asociadas a los fenómenos electromagnéticos (interferencia, difracción, dispersión, etc.) en diferentes áreas, más allá de las antenas lente. 
A continuación, se resumirán las conclusiones principales de esta Tesis, haciendo una división de las áreas temáticas desarrolladas.

\subsubsection{Diseño de antenas lente dieléctricas en Microondas mediante método full-wave}

A partir de las características generales de antenas lente vistas en el Capítulo 4, se analizaron las antenas lente dieléctricas utilizando el código de simulación electromagnética full-wave estudiado en el Capítulo 2. Se estableció un método de diseño de antenas lentes a partir de las simulaciones. La posibilidad de obtener resultados fullwave de las simulaciones permitió obtener múltiples resultados inéditos.

Fue posible determinar el punto focal de las antenas lente dieléctricas con una precisión no alcanzable con los cálculos teóricos hasta ahora aplicados.

Se analizaron diferentes perfiles de lentes y se obtuvieron las principales características de los diagramas de radiación, tales como directividad, ancho del haz principal y relación lóbulo principal a secundario y relación frente espalda. Fue posible comparar las características para los diferentes perfiles de lentes con elevada precisión.

A partir de los diagramas de campo cercano obtenidos de las simulaciones de antenas lente, fue posible detectar un fenómeno electromagnético conocido como Vórtices Ópticos. Dichos fenómenos fueron ampliamente estudiados en el campo de la Óptica, pero no se encontró en la bibliografía referencias de observación de dichos vórtices en el ámbito de las microondas.

Otro resultado importante obtenido es en el análisis del uso de capas adaptativas con el fin de disminuir las pérdidas por reflexión en la lente. Se simularon varios casos de capas adaptativas en lentes dieléctricas y fue posible verificar sus efectos en el diagrama de radiación de la antena lente. Gracias al código full-wave también fue posible visualizar la onda estacionaria formada entre el alimentador y la lente y poder calcular el ROE para cada configuración de capa adaptativa. Se comparó también el efecto de las capas para diferentes perfiles de lentes. 


\subsubsection{Antenas lente planas}

Teniendo en cuenta las últimas tendencias en antenas lente observadas de la bibliografía, fueron investigados otros diseños de antenas lente, las conocidas como antenas planas o de superficies selectivas de fase. Dichos diseños presentan numerosas ventajas comparados con antenas lente dieléctricas. Principalmente requieren un menor espesor por lo que tienen un menor volumen y por lo tanto un peso menor a las lentes dieléctricas.

Fue posible diseñar y construir dos prototipos de antenas lentes planas; la antena plana dieléctrica y la antena de zonas de Fresnel.

La antena lente plana dieléctrica fue diseñada a partir de orificios en un material dieléctrico. Se diseñó y simuló la lente mediante el software de simulación electromagnética Feko 7.0. Dicho diseño permitió construir un prototipo de una antena lente liviana de muy bajo costo. Los resultados obtenidos de las mediciones se ajustan a los obtenidos por simulación.

Otro diseño estudiado fue la lente plana de zonas de Fresnel. La lente de zonas de Fresnel es una lente metálica de muy fácil construcción y de muy bajo costo. Tal como la lente plana dieléctrica, se diseñó y simuló mediante el software de simulación electromagnética Feko 7.0. Fue posible construir un prototipo a partir de las simulaciones y realizar mediciones. Los resultados obtenidos de las mediciones se ajustan muy bien a las simulaciones.

\subsection{Análisis de tiempos de simulación}

A lo largo de la Tesis se aplicaron diversos softwares de simulación para el diseño de antenas lente en microondas. Mediante dichos softwares se emplearon diversos métodos de simulación.

En el Capítulo 4 se utilizó el nuevo código para simular lentes dieléctricas mediante métodos full-wave. Para ello se aplicó una versión 2D de dicho código. Ello permitió obtener resultados muy precisos en muy poco tiempo para lentes de gran diámetro. Dado a que las lentes tienen simetría de revolución, no fue necesario implementar la versión 3D.

Para la simulación de las lentes planas desarrolladas en el Capítulo 5 se utilizó el software comercial FEKO 7.0 mediante métodos asintóticos. Dicho software permite obtener resultados $3 \mathrm{D}$. 
En este apartado se analizan los diferentes tiempos de simulación de los softwares utilizados con el fin de evaluar las diferentes herramientas informáticas en aplicación de antenas lentes de microondas. No es intención hacer un análisis comparativo de rendimiento entre los diferentes softwares ya que los mismos se utilizaron en aplicaciones diferentes.

Todas las simulaciones se hicieron en la misma PC de escritorio con las siguientes características técnicas:

Tabla 7.1: Características de la PC utilizada en las simulaciones.

\begin{tabular}{|l|l|}
\hline Procesador & $\begin{array}{l}\text { Intel Core i7-4790K CPU @ 4,00 } \\
\mathrm{GHz}\end{array}$ \\
\hline Memoria RAM & $16,0 \mathrm{~GB}$ \\
\hline Video & GeForce GTX 970 - 4 GB DDR5 \\
\hline Sistema operativo & Windows 8 - 64 bits \\
\hline
\end{tabular}

En la Tabla 7.2 se resumen los problemas simulados a lo largo de la presente Tesis y su respectivo software aplicado. Asimismo, se exhiben los tiempos de simulación correspondientes.

Tabla 7.2: Tiempos de simulación de todos los problemas simulados.

\begin{tabular}{|c|c|c|c|c|c|c|c|}
\hline & $\begin{array}{c}\text { Lente } \\
\text { simulada }\end{array}$ & $\begin{array}{c}\text { Frecuencia } \\
{[\mathbf{G H z}]}\end{array}$ & $\begin{array}{c}\text { Diámetro } \\
{[\mathbf{m m}]}\end{array}$ & Dim. & $\begin{array}{c}\text { Método } \\
\text { numérico }\end{array}$ & Software & $\begin{array}{c}\text { Tiempo de } \\
\text { simulación } \\
{[\text { [Seg] }}\end{array}$ \\
\hline 5.3 & Hiperbólica & 30 & $300(30 \lambda)$ & $2 \mathrm{D}$ & $\begin{array}{c}\text { full-wave } \\
\text { (MoM) }\end{array}$ & $\begin{array}{c}\text { Código } \\
\text { full-wave }\end{array}$ & 208 \\
\hline 5.3 & Biconvexa & 30 & $300(30 \lambda)$ & $2 \mathrm{D}$ & $\begin{array}{c}\text { full-wave } \\
\text { (MoM) }\end{array}$ & $\begin{array}{c}\text { Código } \\
\text { full-wave }\end{array}$ & 216 \\
\hline 5.4 .3 & $\begin{array}{c}\text { Escalonada } \\
\text { de Fresnel }\end{array}$ & 30 & $300(30 \lambda)$ & $2 \mathrm{D}$ & $\begin{array}{c}\text { full-wave } \\
\text { (MoM) }\end{array}$ & $\begin{array}{c}\text { Código } \\
\text { full-wave }\end{array}$ & 235 \\
\hline 5.4 & Luneberg & 30 & $540(54 \lambda)$ & $2 \mathrm{D}$ & $\begin{array}{c}\text { (ull-wave } \\
\text { (MoM) }\end{array}$ & $\begin{array}{c}\text { Código } \\
\text { full-wave }\end{array}$ & 449 \\
\hline
\end{tabular}




\begin{tabular}{|c|c|c|c|c|c|c|c|}
\hline 5.6 .1 & $\begin{array}{l}\text { Hiperbólica } \\
\text { Sin adaptar }\end{array}$ & 30 & $300(30 \lambda)$ & $2 \mathrm{D}$ & $\begin{array}{l}\text { full-wave } \\
\text { (MoM) }\end{array}$ & $\begin{array}{l}\text { Código } \\
\text { full-wave }\end{array}$ & 208 \\
\hline & $\begin{array}{l}\text { Hiperbólica } \\
\text { Adaptador } \\
\text { simple }\end{array}$ & 30 & $300(30 \lambda)$ & $2 \mathrm{D}$ & $\begin{array}{l}\text { full-wave } \\
\text { (MoM) }\end{array}$ & $\begin{array}{l}\text { Código } \\
\text { full-wave }\end{array}$ & 225 \\
\hline & $\begin{array}{c}\text { Hiperbólica } \\
\text { Adaptador } \\
\text { doble }\end{array}$ & 30 & $300(30 \lambda)$ & $2 \mathrm{D}$ & $\begin{array}{l}\text { full-wave } \\
\text { (MoM) }\end{array}$ & $\begin{array}{l}\text { Código } \\
\text { full-wave }\end{array}$ & 230 \\
\hline 5.7 .1 & $\begin{array}{c}\text { Hiperbólica } \\
\text { biconvexa }\end{array}$ & 100 & $286(95 \lambda)$ & $3 \mathrm{D}$ & $\begin{array}{c}\text { full-wave } \\
\text { (Bor- } \\
\text { MoM) }\end{array}$ & $\begin{array}{l}\text { Grasp } \\
10.6 .0\end{array}$ & 11 \\
\hline 5.7.2 & $\begin{array}{c}\text { Hiperbólica } \\
\text { biconvexa }\end{array}$ & 100 & $286(95 \lambda)$ & $3 \mathrm{D}$ & $\begin{array}{c}\text { Asintótico } \\
\text { (GO-PO) }\end{array}$ & $\begin{array}{l}\text { Grasp } \\
10.6 .1\end{array}$ & 9 \\
\hline 6.3 & $\begin{array}{c}\text { Plana } \\
\text { dieléctrica }\end{array}$ & 23 & $100(7,7 \lambda)$ & $3 \mathrm{D}$ & Asintótico & Feko 7.0 & 14400 \\
\hline 6.4 & $\begin{array}{l}\text { Zonas de } \\
\text { Fresnel }\end{array}$ & 23 & $172(10 \lambda)$ & $3 \mathrm{D}$ & Asintótico & Feko 7.1 & 1200 \\
\hline
\end{tabular}

Como se observa de la Tabla 7.2, se han simulado múltiples lentes bajo diversos métodos de simulación.

Las simulaciones full-wave realizadas con el código en lentes de gran diámetro fueron realizadas en pocos segundos y permitieron observar fenómenos tales como vórtices ópticos en frecuencias de microondas.

La última versión del software comercial Grasp también permite simular lentes de gran tamaño en pocos segundos mediante el método Bor-MOM. Con el software Feko 7.0 no fue posible simular las mismas lentes bajo métodos full-wave con la PC detallada en la Tabla 7.1, pero fue muy útil para simular las lentes planas bajo métodos asintóticos. En particular la lente de zonas de Fresnel dio resultados muy acordes a las mediciones experimentales. 


\subsection{Futuros trabajos}

Como se desprende del estado del arte expuesto en el Capítulo 1, la tendencia actual de las antenas lente se encuentra en el desarrollo de las denominadas lentes planas o transmitarrays. El uso de aplicaciones en frecuencias de las microondas y los $\mathrm{THz}$ hace de las antenas lente una excelente alternativa para lograr antenas altamente directivas de gran ancho de banda.

Asimismo, el diseño de nuevos materiales cada vez más livianos y de menor costo promueve nuevos diseños de lentes. A lo largo de la presente Tesis se han analizado diversas configuraciones de antenas lente, sin embargo, quedan aspectos que sería de interés profundizar en su investigación. Entre ellos pueden mencionarse:

\section{Nuevos diseños de lentes mediante impresoras 3D:}

A medida que las impresoras 3D admiten nuevos materiales y son cada vez más accesibles desde el punto de vista económico, resultan de especial interés en el diseño y construcción de prototipos de lentes [93].

\section{Implementación de la versión 3D del código full-wave:}

En la presente Tesis no fue necesaria la implementación de la versión en 3D del código 3D ya que todas las lentes simuladas cuentan con simetría de revolución y una implementación 3D necesariamente requiere mayor costo computacional. Sin embargo, sería interesante simular lentes dieléctricas que por su diseño no cuenten con simetría de revolución.

\section{Antenas con Metamateriales y superficies selectivas en frecuencia:}

Las nuevas tecnologías tales como la última generación de telefonía celular (5G) exigen cada vez antenas de mayor ganancia [94]. Para lograr dicho objetivo una de las últimas tendencias es la implementación de metamateriales y superficies selectivas en frecuencia. Las últimas versiones de los softwares comerciales presentan herramientas que permiten diseñar y construir dicho tipo de superficies.

\section{Antenas lentes en aplicaciones en los THz:}

Aplicaciones en radioastronomía y ciencia espacial en frecuencias de $\mathrm{THz}$ típicamente requieren la operación de instrumentos de imágenes y espectroscopía en anchos de banda muy grandes [95].

La relación entre el tamaño de las lentes y la longitud de onda para dichas aplicaciones hace que para obtener resultados precisos se requiera de elevado costo 
computacional. Por lo que sería fundamental continuar profundizando sobre la aplicación de los diferentes métodos computacionales con el fin de obtener resultados precisos con computadoras personales 


\section{Bibliografía}

[1] R. Sauleau, C. A. Fernandes, J. R. Costa. Review of Lens Antenna design and technologies for MM-wave shapedbeam applications". 11th International Symposium on Antenna Technology and Applied Electromagnetics [ANTEM 2005].

[2] E. Hecht. “Optics fifth edition”. Pearson Education, 2017.

[3] D. B. Davidson. "Computational Electromagnetics for RF and Microwave Engineering”, Cambridge University Press, 2005.

[4] F. Vico, L. Greengard, M. Ferrando. "Fast convolution with free-space Green's functions", Journal of Computational Physics, vol. 323, 15 October 2016.

[5] Kriss, Timothy, V. Martich. "History of the Operating Microscope: From Magnifying Glass to Microneurosurgery”. Neurosurgery. April 1998.

[6] Pliny the Elder. "Natural History".

[7] O.J. Lodge, J.L. Howard. "On Electric Radiation and its Concentration by Lenses". Proceedings of the Physical Society of London. 1888

[8] R. K. Luneburg, M. Herzberger. "Mathematical theory of optics", Brown University. Graduate School. Providence, R.I. 1944,

[9] W. E. Kock. "Metal-Lens Antennas", Proceedings of the I.R.E. and Waves and Electrons, noviembre 1946.

[10] A. N. Whitehead. "The theory of parallel-plate media for Microwave lenses", Journal of the Institution of Electrical Engineers. 1951.

[11] J. E. Eaton. "On Spherically Symmetric Lenses", Transactions of the IRE Professional Group on Antennas and Propagation, December 1952.

[12] G. D. Peeler. "A Two-Dimensional Microwave Luneberg Lens", Transactions of the IRE Professional Group on Antennas and Propagation. Volume: 1 , Issue: 1 , July 1953.

[13] K. Kay. "Electromagnetic Theory and Geometrical Optics", Interscience Publishers, New York. 1965. 
[14] A. Olver, P. Clarricoats, A. Kishk, L. Shafai, "Microwave Horns and Feeds", Chap. 11, IEEE Press, New York. 1998.

[15] K. Kelleher. "Scanning Antennas", Chap 15 in Antenna Engineering Handbook, H.Jasik (Ed.), McGraw-Hill, New York. 1961.

[16] C. A. Fernandes, V. Brankovic; S. Zimmermman; M. Filipe, L. Anunciada. "Dielectric Lens Antenna for Wireless Broadband Communications", Wireless Personal Communications, Vol. 10, No. 1, pp. 19 - 32, June, 1999.

[17] C.A. Fernandes. "Shaped dielectric lenses for wireless millimeter-wave communications". Antennas and Propagation Magazine, IEEE 41:141-150. 1999.

[18] C. Salema, C.A. Fernandes, R. Jha. "Solid Dielectric Horns", Chap. 7, Artech House, Boston. 1998.

[19] C.A. Fernandes, L.M. Anunciada. "Constant flux illumination of square cells for millimeter-wave wireless communications". Microwave Theory and Techniques, IEEE Transactions on 49:2137-2141. 2001.

[20] R. Sauleau, B. Bares. "A complete procedure for the design and optimization of arbitrarily shaped integrated lens antennas". Antennas and Propagation, IEEE Transactions on 54:1122-1133. 2006

[21] B. Bares, R. Sauleau. "Design and optimisation of axisymmetric millimetrewave shaped lens antennas with directive, secant-squared and conical beams". Microwaves, Antennas \& Propagation, IET 1:433-439. 2007.

[22] S. Cornbleet. "Microwave and Geometrical Optics", Academic Press, London. 1994.

[23] K. Hongo. H. Kobayashi "Radiation characteristics of a plano-convex lens antenna". Radio Science Volume 31, Number 5, Pages1 025-1035, September-October 1996.

[24] P. Piksa, S. Zvanovec, P. Cerny, "Elliptic and Hyperbolic dielectric lens antennas in MM-waves”. RADIOENGINEERING, VOL. 20, NO. 1, APRIL 2011.

[25] C. D. Diallo, O. Quevedo-Teruel, G. Valerio, H. Legay, R. Sauleau. "ParallelPlate-Waveguide Luneburg Lens Through a Holey Plate Metasurface". 9th European Conference on Antennas and Propagation (EuCAP), 2015. 
[26] Z. Shi, S. Yang, L. Zhou, Y. Chen. "A Dual Circularly Polarized Planar Luneberg Lens Antenna for mmwave Wireless Communication”. 978-1-5090-2017IEEE. 2016.

[27] M. I. Vill'a "Design and Performance Evaluation of Millimeter-Wave Flat Lens Antennas for Communications, Radar and Imaging Applications". Phd Thesis program on Signal Theory and Communications, AntennaLab Research Group, Barcelona, July 2016.

[28] J. C. Wiltse, "Fresnel Zone-Plate Lenses". Proceedings of SPIE, Vol. 544, Millimeter-Wave Technology III, July 1985.

[29] H. D. Hristov, "Fresnel Zones in Wireless Links, Zone Plate Lenses and Antennas", Artech House, Norwood, MA, 2000.

[30] N. Gagnon, A. Petosa, and D. A. McNamara, "Comparison between Conventional Lenses and an Electrically Thin Lens Made Using a Phase Shifting Surface (PSS) at Ka Band," Loughborough Antennas \& Propagation Conference, pp.117-120, 16-17, Loughborough, UK. November 2009.

[31]S. Zhang, W. Whittow, Y. Vardaxoglou. "3D-printed Fresnel zone plate lens". International Symposium on Antennas and Propagation (ISAP). 2016.

[32] Pei-Ling Chi ; Chi-Hsien Pao ; Ming-Hui Huang. "High-Gain Patch-Fed 3DPrinting Fresnel Zone Plate Lens Antenna for $60-\mathrm{GHz}$ Communications". IEEE International Symposium on Antennas and Propagation \& USNC/URSI National Radio Science Meeting. 2018.

[33] J. Pourahmadazar, S. Sahebghalam, S. A.i Aghdam, M. Nouri. "A Millimeter-Wave Fresnel Zone Plate Lens Design Using Perforated 3D Printing Material". IEEE MTT-S International Microwave Workshop Series on Advanced Materials and Processes for RF and THz Applications (IMWS-AMP). 2018.

[34] J. M. Rodríguez ; Hristo D. Hristov ; W. Grote. "Fresnel zone plate and ordinary lens antennas: Comparative study at microwave and terahertz frequencies". 41st European Microwave Conference. 2011.

[35] Llombart, N., Chattopadhyay, G., Skalare, A., \& Mehdi, I. "Novel terahertz antenna based on a silicon lens fed by a leaky wave enhanced waveguide". IEEE Transactions on Antennas and Propagation. 2011. 
[36] Ling-Yun Li ; Tian-Yu Pen ; Hao Sun. "The Influence of Geometries on Terahertz Metal Grid Metamaterial lens". IEEE Asia-Pacific Conference on Antennas and Propagation (APCAP). 2018.

[37] X. Xu, X. Zhang, Lei Surr, K. Wang. “A Terahertz Antenna Loaded with Dielectric Lens". International Conference on Microwave and Millimeter Wave Technology (ICMMT). 2018.

[38] D. M. Hailu, I. A. Ehtezazi, S. Safavi-Naeini. "Fast Analysis of Terahertz Integrated Lens Antennas Employing the Spectral Domain Ray Tracing Method". IEEE Antennas and Wireless Propagation Letters. 2008.

[39] Yurduseven, Ozan. "Wideband Integrated Lens Antennas for Terahertz Deep Space Investigation”. Phd Thesis. Delft University of Technology. 2016.

[40] B. A. Munk. "Frequency Selective Surfaces: Theory and Design". John Wiley and Sons Inc., New York. 2000.

[41] Y. Fan, B. Ooi, H. D. Hristov, and M. Leong. "Compound diffractive lens consisting of Fresnel zone plate and frequency selective screen." IEEE Trans. Antennas Propag., vol. 58, no. 6, pp. 1842-1847, Jun. 2010.

[42] O. Malyuskin, V. Fusco, and A. Schuchinsky, "Phase conjugating wire FSS lens”. IEEE Trans. Antennas Propag., vol. 54, no. 5, pp. 1399-1404. Jun. 2006.

[43] M. Al-Joumayly and N. Behdad, "Wideband planar microwave lenses using sub-wavelength spatial phase shifters.” IEEE Trans. Antennas Propag., vol. 59, no. 12, pp. 4542-4552. Dec. 2011.

[44] M. Li, N. Behdad. "Wideband true-time-delay microwave lenses based on metallo-dielectric and all-dielectric lowpass frequency selective surfaces". IEEE Trans. Antennas Propag., vol. 61, no. 8, pp. 4109-4119. Aug. 2013.

[45] A. H. Abdelrahman, A. Z. Elsherbeni, and F. Yang. "Transmission phase limit of multilayer frequency selective surfaces for transmitarray designs". IEEE Trans. Antennas Propag., vol. 62, no. 2, pp. 690-697, Feb. 2014.

[46] J. Thornton, Kao-Cheng Huang. "Modern lens antennas for communications engineering”. IEEE press. Wiley. 2013.

[47] C. A. Fernandes, E. B. Lima, J. R. Costa. "Dielectric Lens Antennas". Handbook of Antenna Technologies. 2016. 
[48] W. C. Gibson. "The Method of Moments in Electromagnetics". Chapman \& Hall/CRC. 2008.

[49] H. Gómez Sousa. "Métodos de electromagnetismo computacional para análisis de estructuras arbitrarias y su aplicación a problemas de nanoimaging”. Tesis. Universidad de Vigo (España). 2015.

[50] R. F. Harrington. "Field Computation by Moment Methods". IEEE press. 1992

[51] Altair HyperWorks FEKO, Altair Engineering Inc., Address: 1820 E. Big Beaver Rd. Troy MI 48083, United States. http://www.altairhyperworks.com/product/FEKO.

[52] Ticra. GRASP. https://www.ticra.com/software/grasp/.

[53] P. Meincke, E. Jørgensen. "Efficient Body of Revolution Method of Moments for Rotationally Symmetric Antenna Systems with Offset Illumination". IEEE Antennas and Propagation Society International Symposium (APSURSI). 2014.

[54] [1] J. R. Mautz, R. F. Harrington. "H-field, E-field, and combined field solutions for bodies of revolution". Syracuse University, NY, USA, Tech. Rep. TR-77-2. Feb. 1977.

[55] T. K. Wu, L. L. Tsai. "Scattering from arbitrarily-shaped lossy dielectric bodies of revolution" Radio Sci., vol. 12, pp. 709-718, 1977.

[56] [3] A. A. Kishk, L. Shafai. "Different formulations for numerical solution of single or multibodies of revolution with mixed boundary conditions". IEEE Transactions on Antennas and Propagation, vol. 34, no. 5, pp. 666-673. 1986.

[57] E. Jørgensen, P. Meincke, M. Sabbadini. "Fast and accurate design tool for rotationally symmetric reflector antennas with $3 \mathrm{D}$ waveguide components and support structures". 34th ESA Antenna Workshop, Noordwijk, The Netherlands. 2012.

[58] E. Jørgensen, P. Meincke. "Domain-decomposition technique for efficient analysis of rotationally symmetric reflector systems containing 3D structures". 7th European Conference on Antennas and Propagation (EuCAP), pp. 1826 - 1830. 2013.

[59] E. Jørgensen, J. L. Volakis, P. Meincke, O. Breinbjerg. "Higher order hierarchical Legendre basis functions for electromagnetic modeling". IEEE Transactions on Antennas and Propagation, vol. 52, no. 11, pp. 2985-2995. Nov. 2004.

[60] Matlab. https://www.mathworks.com/products/matlab.html. 
[61] Á. Cardama Aznar, L. J. Roca, J. M. Rius Casals, J. R. Robert, S. Blanch Boris, M. Ferrando Bataller. “Antenas”. EDICIONS UPC. Segunda edición. Septiembre de 2002.

[62] J. A. Bava. “Antenas Reflectoras en microondas”. Facultad de Ingeniería UNLP. Libros de Cátedra. Editorial de la Universidad Nacional de La Plata. 2013.

[63] J. A. Bava. "Antenas para radiómetros en onda milimétrica con haces múltiples”. Tesis doctoral. Facultad de Ingenieria - UNLP. 2010.

[64] T. A. Milligan. "Modern Antenna Design”. Mc Graw Hill Book Company. 2005 .

[65] C. Balanis. "Antenna Theory Analysis and Design". John Wiley Sons, Inc. 2005 .

[66] W. L. Stuzman, G. A Thiele. "Antenna Theory and Design”. John Wiley \& Sons. Inc. 1998.

[67] C. Balanis. "Modern Antenna Handbook”. Wiley. 2008.

[68] P. M. Gross, J. Vernieri, F. Vico, A. Bava, M. F. Bataller, J. R. Robert. "Aplicación de nuevo código de simulación para estudio de lentes en microondas en 2D". XXXII Simposium Nacional de la Unión Científica Internacional de Radio (URSI 2017). 6 a 8 de Septiembre de 2017. Cartagena, España.

[69] P. M. Gross, J. Vernieri, F. Vico, A. Bava, M. F. Bataller. "Lens Antennas focus determination using Full-Wave simulation". 12th European Conference on Antennas and Propagation (Eucap2018). 9 al 13 de Abril de 2018. Londres, Reino Unido.

[70] P. M. Gross, J. Vernieri, F. Vico, A. Bava, M. F. Bataller. “Análisis de las características de antenas lentes en microondas mediante método full-wave". IEEE Argencon 2018. 6 al 8 de junio de 2018. San Miguel de Tucumán. Tucumán. Argentina.

[71] P. M. Gross, J. Vernieri, F. Vico, A. Bava, M. F. Bataller. "Simulación de antenas lente en microondas". 102 ${ }^{a}$ Reunión Nacional de la Asociación Física Argentina. 26 al 29 de Septiembre de 2017. La Plata, Buenos Aires, Argentina.

[72] P. M. Gross, J. Vernieri, F. Vico, A. Bava, M. F. Bataller. "Análisis en frecuencia de lente de Fresnel en microondas mediante simulación Full-Wave". XXXIII Simposium Nacional de la Unión Científica Internacional de Radio (URSI 2018). 5 a 7 de Septiembre de 2018. Granada, España. 
[73] A. Ya. Bekshaev, A. I. Karamoch. "Structure of optical vortices produced by holographic gratings with "fork" geometry: Kummer beams". Journal of Holography and Speckle. June 2007.

[74] Brijesh Kumar Singh, G. Singh,2 P. Senthilkumaran, D. S. Mehta. "Generation of Optical Vortex Arrays Using Single-Element Reversed-Wavefront Folding Interferometer”. International Journal of Optics, Article ID 689612. Volume 2012.

[75] Basistiy, V A Pas'ko, V V Slyusar, M S Soskin and M V Vasnetsov. "Synthesis and analysis of optical vortices with fractional topological charges". Journal of Optics A: Pure and Applied Optics, Volume 6, Number 5. 19 April 2004.

[76] Freddy Monroy y Lyubov Kreminskaya. "Creación de Vortices Ópticos en la interferencia de múltiples haces”. Revista Hallazgos. Vol. 3, Núm. 5. 2006.

[77] P. M. Gross, J. Vernieri, F. Vico, A. Bava, M. F. Bataller. "Estudio de vórtices ópticos en antenas lente". XVII Reunión de trabajo en Procesamiento de la Información y Control (RPIC 2017). 20 a 22 de septiembre de 2017. Mar del Plata, Argentina.

[78] P. M. Gross, J. Vernieri, G. Rodriguez. "Simulaciones Full-Wave en la banda de microondas". 5tas Jornadas de Investigación y Transferencia. 9 al 11 de Abril 2019. Facultad de Ingeniería. UNLP. La Plata, Argentina.

[79] P. C. Hargrave1, G. Savini, N. Trappe, A. Challinor, S.B. Sørensen, P. A. R. Ade, R. V. Sudiwala, I. K. Walker, M. Gradziel, N. Tynan, M. van der Vorst. "Coated Dielectric Lens Design, Modelling and Measurements for Future CMB Polarimetry Missions”. 7th European conference on Antennas and Propagation (Eucap). 2013.

[80] D. Cheng. "Fundamentos de electromagnetismo para ingeniería". Pearson. 1998.

[81] P. M. Gross, J. Vernieri, F. Vico, A. Bava, M. F. Bataller. "Estudio de adaptación en antenas lente dieléctricas mediante simulación full-wave". XVIII Reunión de trabajo en Procesamiento de la Información y Control. Bahia Blanca, Argentina. 18 al 20 de septiembre. 2019.

[82] D. M. Pozar. "Flat Lens Antenna Concept Using Aperture Coupled Microstrip Patches”. Electron. Lett. Vol. 32, No. 23, pp. 2109-2111. November 1996.

[83] N. Gagnon, A. Petosa, D. A. McNamara. "Research and developmenton phase-shifting surfaces (PSSs)". IEEE Transactions on Antennas and Propagation. Mag., vol. 55,no. 2, pp. 29-48. Jun. 2013. 
[84][ M. A. Al-Joumayly, N. Behdad. "Wideband planar microwave lensesusing sub-wavelength spatial phase shifters." IEEE Transactions on Antennas and Propagation, vol. 59, no. 12, pp. 4542-4552, Nov. 2011.

[85] C. Pfeiffer and A. Grbic, "Millimeter-wave transmitarrays for wavefrontand polarization control," IEEE transactions on microwave theory and techniques., vol. 61,no. 12, pp. 4407-4417. Nov. 2013.

[86] A. Abbaspour-Tamijani, K. Sarabandi, and G. M. Rebeiz, "Antenna-filterantenna arrays as a class of bandpass frequency-selective surfaces". IEEE transactions on microwave theory and techniques, vol. 52, no. 8, pp. 1781-1789. Aug.2004.

[87] Marc Imbert VILLA. "Design and Performance Evaluation of MillimeterWave Flat Lens Antennas for Communications, Radar and Imaging Applications". Ph.D. program on Signal Theory and Communications. 2016.

[88] A. Moknache, L. Dussopt, J. Säily, A.Lamminen, M. Kaunisto, J. Aurinsalo. "A switched-beam linearly-polarized transmitarray antenna for V-band backhaul applications". 10th European Conference on Antennas and Propagation (EuCAP). 2016.

[89] D. A. Roper, B. L. Good, R. Mccauley, S. Yarlaggada, J. Smith, A. Good, P. PA, M. Smiritznik. "Additive manufacturing of graded dielectrics". IOP Publishing, Smart Mater. Struct. 23045029 (9pp). 2014.

[90] P. M. Gross, J. P. Ciafardini, J. Vernieri, A. Bava. “Antena lente plana dieléctrica". X Congreso Argentino de Tecnología Espacial. Ciudad autónoma de Buenos Aires, Argentina. 10-12 Abril, 2019

[91] H. D. Hristov. "Terahertz Harmonic Operation of Microwave Fresnel Zone Plate Lens and Antenna: Frequency Filtering and Space Resolution Properties". International Journal of Antennas and Propagation Volume 2011, Article ID 541734, 8 pages. 2011.

[92] P. M. Gross, J. P. Ciafardini, F. Vico, A. Bava, M. F. Bataller. “Antenas lente con metamateriales y su aplicación en tecnología espacial". IX Congreso Argentino de Tecnología Espacial. Córdoba, Argentina. 26-28 Abril, 2017.

[93] R. J. Friel, M. Gerling-Gerdin, E. Nilsson, B. P. Andreasson. “3D Printed Radar Lenses with Anti-Reflective Structures”. Designs (ISSN 2411-9660). 2019.

[94] Bo He, Yong-Chang Jia, Huan He. "Design of High-Gain Lens Antenna for 5G Application". Sixth Asia-Pacific Conference on Antennas and Propagation (APCAP). 2017. 
[95] O. Yurduseven, N. Llombart, A. Neto, J. Baselmans. "A Dual Polarized Antenna for THz Space Applications: Antenna Design and Lens Optimization”. IEEE Antennas and Propagation Society International Symposium (APSURSI). 2014. 